Departamento de Arquitectura, Construcción y Sistemas Oceánicos y Navales Escuela Técnica Superior Ingenieros Navales

Universidad Politécnica de Madrid

PhD Thesis

\title{
Design of a physical and interactive real-time Simulator based on a dynamic VPP as a support tool for sailing yacht design and operation
}

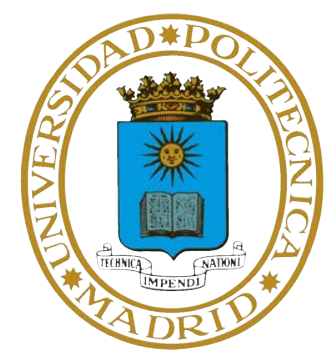

Ignacio Castañeda Sabadell

M. Sc. in Naval Architecture, Marine and Ocean Engineering

Supervisors:

Manuel Ruiz de Elvira

Ph.D. in Naval Architecture

Francisco Pérez Arribas

Ph.D. in Naval Architecture 
“J'écoute la mer, j'écoute le vent, j'écoute les voiles qui parlent avec la pluie

et les étoiles dans les bruits de la mer et je n'ai pas sommeil."

Bernard Moitessier 


\section{Acknowledgements}

I would like to thank the following people for their assistance and support during the $\mathrm{PhD}$ :

- To my supervisor Manuel Ruiz de Elvira for all the knowledge, experience, help, resources and support he brought to me constantly during the whole process (from the first idea to the last word in the text). He really helped me deeply understand how a sailing boat works. Without his help, this work would not be possible.

- To my supervisor Francisco Pérez Arribas for all the help and support he brought to me during the development of the thesis and for all the help and comments he gave me when teaching together in the UPM.

- To Prof. Ricardo Zamora, the person that introduced me to the technical perspective of sailing yachts, allowing me to discover this beautiful side of sailing.

- To Prof. Antonio Souto, for providing some of the resources needed for the development of the thesis.

- To Mike and Caz Drummond, who proofread tirelessly the thesis to the very last moment, helping me any time I needed and improving the thesis quality thanks to their corrections, comments and suggestions. I also need to thank Mike for sharing with me technical comments and suggestions, helping me to understand a lot of different aspects of this work.

- To Prof. Richard G.J. Flay and all the people working on the TFWT of the University of Auckland for their warm welcome to the wind tunnel and for sharing with me their knowledge on how sails and wind tunnels work. The development of specific parts of this work would not be possible without them. They also gave me the opportunity to go to New Zealand and discover such an amazing place and people. 
- To Andrew Mason who guided me when I was starting to work with $\mathrm{VR}$ engines and helped me to understand and know the different options available for the simulator.

- To José Luis Vela and Javier Cuevas who gave me ideas and advice on how the simulator has to be developed and built, helping me discover different strategies for the simulator implementation.

- To the former director of the CEHINAV, Prof. Luis Pérez Rojas and all the people working there, especially to Patricia Alcanda, Javier Calderón, Elkin Botia and José Luis Cercós Pita, for all their help and support, and for looking after me during the whole process.

- To my PhD colleagues from the University of Auckland for all the help they brought to me and all the great moments we shared together during my research stay.

- To Benoit Marie for helping me to understand the Moth foiler sailboat and for sharing with me important information about it.

- To my group of friends of Devonport in Auckland, for their warm welcome and the great times we shared together during my research stay in New Zealand, becoming almost my family there when I was far away from home.

- To my Hydraulink sailing friends, for letting me sailing and share fun times with them, making my research stay more enjoyable and interesting.

- To the international experts Richard Luco, Dan Walker and especially to Jerome H. Milgram, who reviewed the draft and provided insightful comments and advice that improved the final version of this work.

- A mis padres, hermana y amigos por aguantar mi trabajo y preocupaciones a lo largo de toda la tesis, dándome ánimos y apoyo. Me gustaría agradecer también a Ana, que me ha acompañado a lo largo de todo este proceso, y a su familia por haberme aguantado y ayudado durante la consecución de este trabajo. 


\begin{abstract}
Performance assessment and training are two major components in technified high-level sports. Recently, high-performance sailing competitions have been forced to change with the use of the foiling technology and the training restrictions, more often included within competition rules. To answer those new situations, a new design and training tool has to be developed. Up until now, the performance assessment was made calculating the balance of forces and moments using a Velocity Prediction Program (VPP). This procedure was more than capable of dealing with conventional displacement sailboats but the dynamic nature of foiling boats compelled the development of a time-domain VPP for dynamic analysis towards a better performance. In order to improve the dynamic analysis capabilities of the VPP and to add the ability for it to be used for training, a new tool is proposed to fulfil those requirements: the simulator.

Throughout this work the concept of the simulator has been discussed identifying its fundamental components, namely:

- The time-domain VPP.

- The motion Platform.

- The visualization system.

- The physical user interface for the simulator operation.

The different uses and applicability for this concept are explored in order to identify the potential benefits of such a tool in a high-performance environment. The different components are analysed, identifying specific problems in each area and proposing different solutions. All of this work allows proposing a modular framework of a simulator to be used in highperformance sailing boat design and training.
\end{abstract}




\section{Resumen}

La evaluación del rendimiento y el entrenamiento son dos de los componentes principales en los deportes tecnificados de alto nivel. Recientemente, las competiciones de vela de alto rendimiento se han visto obligadas a cambiar con la aparición y el uso de los "foils" y las restricciones de entrenamiento, incluidas más a menudo dentro de las reglas de las competiciones. Para responder a estas nuevas situaciones, se debe desarrollar una nueva herramienta de diseño y entrenamiento. Hasta ahora, la evaluación del rendimiento en barcos de vela se realizaba calculando el equilibrio de fuerzas y momentos utilizando un programa de predicción de velocidad (VPP). Este procedimiento es más que capaz con veleros convencionales, pero la naturaleza dinámica de los barcos con "foils" ha obligado al desarrollo del VPP en el dominio del tiempo para permitir el análisis dinámico de este tipo de barcos en busca de un mayor rendimiento. Con el fin de mejorar las capacidades de análisis dinámico del VPP y agregar la capacidad de ser utilizado durante el entrenamiento, se propone una nueva herramienta que cumple con esos requisitos: el simulador.

A lo largo de este trabajo, se ha discutido el concepto del simulador identificando sus componentes fundamentales, a saber:

- El VPP en el dominio del tiempo.

- La plataforma de movimiento.

- El sistema de visualización.

- La interfaz de usuario física para la operación del simulador.

Se exploran los diferentes usos y la aplicabilidad de este concepto para identificar los beneficios potenciales de una herramienta de este tipo en un entorno de alto rendimiento. Los diferentes componentes son analizados, identificando problemas específicos en cada área y proponiendo diferentes soluciones. Todo este trabajo permite proponer un simulador 
como entorno modular para ser utilizado en el diseño y entrenamiento en competiciones de vela de alto rendimiento. 


\section{Contents}

Glossary $\quad 2$

1 Overview 4

1.1 Background and Context . . . . . . . . . . . . . . . 4

1.2 The Simulator: A Definition . . . . . . . . . . . . 5

1.3 State-of-the-Art . . . . . . . . . . . . . . . 6

1.4 Contributions . . . . . . . . . . . . . . . . . 9

1.5 Outline of the Thesis . . . . . . . . . . . . . . . . 10

2 The Sailing Yacht Simulator $\quad 12$

2.1 A New Design Asset . . . . . . . . . . . . . . . . . . . 13

2.1.1 Advanced Performance Assessment . . . . . . . . . . . . . 13

2.1.2 Early Testing of Design Features . . . . . . . . . . . . . 13

2.1.3 Assistance to Energy Management . . . . . . . . . . . . . . . 14

2.1.4 Continuous Dynamic VPP Verification . . . . . . . . . . . . . 14

2.1.5 Two Boat Performance Evaluation with Only One Real Boat . 14

2.2 The Perfect Testing Platform . . . . . . . . . . . . . . . 15

2.2.1 No Dependency on Weather Conditions . . . . . . . . . . . 15

2.2.2 Evaluate Safety Limits and Corrections . . . . . . . . . . . . 15

2.2.3 Evaluation of Optimal Response to Soft Transitions and Patterns of Changes . . . . . . . . . . . . . . . . 15

2.2.4 Evaluation of Situations with Similar Performances but Different Executions . . . . . . . . . . . . . . . . 16

2.2.5 Evaluate Early Signs for Required Corrections . . . . . . . . . 16

2.2 .6 Test Deck Gear . . . . . . . . . . . . . . . . . . . . . 16

2.2.7 Provide a Test Platform for Augmented Reality . . . . . . . . 17

2.3 A New Interface for Improved Communications Between Athletes and Designers . . . . . . . . . . . . . . . . . 18 
2.4 A Sailor's Training Tool . . . . . . . . . . . . . . . . . . . . . 19

2.4.1 Assistance Towards a Steeper Learning Curve . . . . . . . . 19

2.4.2 Training for Subsequent On-The-Water Tests . . . . . . . 19

2.4 .3 Race Preparation . . . . . . . . . . . . . . . . . . . 20

2.4 .4 Model Racing Situations . . . . . . . . . . . . . . . . . 20

2.4.5 Training of Natural Reactions . . . . . . . . . . . . . 21

2.4.6 Training Anticipation Regarding what to Expect from Unusual Situations . . . . . . . . . . . . . . 21

2.4.7 Anticipate the Behaviour of a New Design or Type of Yacht . 21

2.4.8 Virtual Repetition of Real On-The-Water Training and Regattas 22

2.5 A Modular Approach of the Sailing Yacht Simulator . . . . . . . . . 22

2.6 The Concepts of Immersion, Presence and Fidelity . . . . . . . . . . . 24

3 PASim, the Simulator Core $\quad 26$

3.1 The VPP as the Heart of a 6-DOF Sailing Simulator . . . . . . . 26

3.2 From a Static Equilibrium to a Time-Domain Calculation . . . . . . . 27

3.3 Physical Models for Forces and Moments . . . . . . . . . . . . 30

3.3 .1 Interpolated Models . . . . . . . . . . . . . . . . 31

3.3.1.1 Experimental Data . . . . . . . . . . . . . . . 32

$3.3 .1 .2 \quad$ Numerical Data. . . . . . . . . . . . . . . . . . 36

3.3.1.3 Special Considerations . . . . . . . . . . . . . . . 39

3.3.2 Analytical Models . . . . . . . . . . . . . . . . . . . . . . 40

3.3.2.1 Analytical Models . . . . . . . . . . . . . . 41

3.3.2.2 Numerical Methods Solved in Execution Time . . . . 42

3.3.2.3 Numerical Methods Combined with Interpolation Schemes 44

3.3.2.4 Unsteady Model Correction . . . . . . . . . . . . 45

3.3 .3 Other Models . . . . . . . . . . . . . . . . . . . . . 47

3.4 Time-Domain Solver Numerical Schemes . . . . . . . . . . . . . . 48

3.5 Autopilots . . . . . . . . . . . . . . . . . . 53

3.5.1 Boat Elements . . . . . . . . . . . . . . . 55

3.5 .2 Algorithms . . . . . . . . . . . . . . . . 57

3.5.3 A General Approach for Neural-Network-Based Autopilots . . 59

3.5.4 The Simulator as a Safe Test Environment for Control Systems 59

3.5.5 Use of Autopilots to Improve Performance . . . . . . . . . . 60

3.5.6 Unstable Boats . . . . . . . . . . . . . . . . . . 60 
3.6 A Note on Multi-Threading Execution and Multi-Computer Configu-

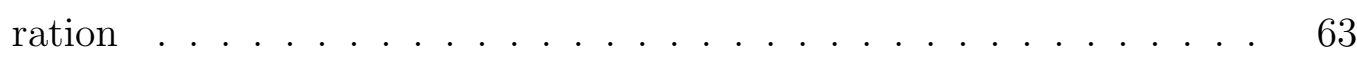

3.7 An Example : The Moth Foiler . . . . . . . . . . . . . . . 65

3.7 .1 The Boat . . . . . . . . . . . . . 66

3.7.2 Forces and Moments Models . . . . . . . . . . . . 67

3.7.3 An Example of the Foiling Moth Simulator Usage: The Transition Study From Non-Foiling to Foiling . . . . . . . . . 70

4 The Motion Platform $\quad 73$

4.1 The Degrees of Freedom . . . . . . . . . . . . . . . . 74

4.1.1 Conventional Monohulls and Slow Multihulls . . . . . . . . . . 74

4.1.2 Fast Multihulls and Foiling Boats . . . . . . . . . . . . 75

4.2 Reproduction of Motion and Position Versus Reproduction of Rotational Velocities and Linear Accelerations . . . . . . . . . . . . 76

4.2.1 A Tool to Reproduce Motions and Position . . . . . . . . . . . 77

4.2.2 A Tool to Reproduce Rotational Velocities and Linear Accelerations .......................... 78

4.3 The Stewart Platform: A Six Degrees of Freedom Parallel Robot . . . 80

4.3.1 Description . . . . . . . . . . . . . . . . . . 81

4.3.2 Motion Cue Generation and Analysis Algorithms . . . . . . . 82

4.3.2.1 Inverse Kinematics . . . . . . . . . . . . . . . . 83

4.3.2.2 Direct Kinematics . . . . . . . . . . . . . . . . . . 84

4.3.2.3 Inverse Dynamics . . . . . . . . . . . . . . . . 84

4.3 .3 Limitations . . . . . . . . . . . . . . . . 86

4.4 Washout Algorithm . . . . . . . . . . . . . . . . . 87

4.4.1 General Description . . . . . . . . . . . . . . . . . 87

4.4.1.1 Classical Washout . . . . . . . . . . . . 87

4.4.1.2 Optimal Washout . . . . . . . . . . . . . 90

4.4.1.3 Adaptive Washout . . . . . . . . . . . . . . . . 92

4.4.1.4 Notes on the Implementation . . . . . . . . . . . . 94

4.4.2 Washout Algorithm Tuning . . . . . . . . . . . 95

4.4.2.1 Vestibular Model . . . . . . . . . . . . . . . . . 95

4.4.2.2 Genetic Algorithms . . . . . . . . . . . . . . . 97

4.4.2.3 Time History Information Generation for Coefficients Training . . . . . . . . . . . . . . 98 
4.4.2.4 Filtering Parameter Selection Using a Vestibular Model and Genetic Algorithms Training . . . . . . 100

5 Visualisation Interface 103

5.1 Vision as the Main Source for Motion Simulation . . . . . . . . . 103

5.2 The Virtual Reality Engine to Represent the Optic Flow . . . . . . 105

5.2 .1 Simulator Needs . . . . . . . . . . . . . . . . . . . . . . . 105

5.2.2 A Tool to Generate Environmental Cues . . . . . . . . . . 108

5.2 .3 A Tool to Show Vital Information . . . . . . . . . . . . . 112

5.2.4 A Tool that Improves Two Boat Interaction Awareness . . . . 114

5.3 Specific Challenges of a Realistic Visual Experience . . . . . . . . . 115

5.4 Virtual Reality Representation Systems . . . . . . . . . . . . . . . 119

6 The Physical User Interface 122

6.1 A User Interface to Allow the Simulator Operation . . . . . . . . . 122

6.2 From a Simple Control to a More Realistic Experience . . . . . . . 123

6.2 .1 Steering Elements . . . . . . . . . . . . . . . . 125

6.2 .2 Sails Operation . . . . . . . . . . . . . . . . . . 126

6.2 .3 User Position . . . . . . . . . . . . . . . . . . . . . 127

6.2.4 New Interface Systems and Other Settings Adjustment . . . . 128

6.2.5 Cockpit Reproduction . . . . . . . . . . . . . . . . 129

$\begin{array}{lll}7 & \text { Conclusions } & 130\end{array}$

A AC72 Dynamic Data Time History Plots 135

A.1 Time History Comparison for Different Numerical Schemes . . . . . . 136

A.2 Time History Comparison Using Euler and Midpoint Methods with Different Time Steps . . . . . . . . . . . . . . . . . . . . 144

B Time History Data of the Simulated Foiling Moth Runs 152

B.1 Time History Data of the Simulated Foiling Moth Run Using a Heel PID Controller . . . . . . . . . . . . . . . . . . 152

B.2 Time History Data of the Simulated Foiling Moth Transition Run . . 158

C Multi-Threading Tests Time Results 165 
$\begin{array}{lr}\text { D Moth Foiler Models } & 169\end{array}$

D.1 Aerodynamic Models . . . . . . . . . . . . . . . . . . . . . 169

D.1.1 Sails . . . . . . . . . . . . . . . . . . 169

D.1.2 Windage ...................... 171

D.2 Hydrodynamic Models . . . . . . . . . . . . . . . . . . . . 171

D.2.1 Lifting Surfaces . . . . . . . . . . . . . . . . . . 171

D.2.2 Hull . . . . . . . . . . . . . . . . . . . . . . . . 173

D.3 Other Models . . . . . . . . . . . . . . . . . . . . . . 174

D.3.1 Control Models . . . . . . . . . . . . . . . . . . . . . . 174

D.3.2 Other Hydrostatics and Weight Stability Models . . . . . . . . 174

E Washout Tuning Algorithm Results 176

$\begin{array}{lr}\text { Bibliography } & 181\end{array}$ 


\section{List of Figures}

2.1 Simulator general diagram of the modular implementation. . . . . . . 24

3.1 CEHINAV Towing Tank Facility in the ETSIN-UPM in Madrid. Photograph by CEHINAV Research Group . . . . . . . . . . . . . . 33

3.2 Twisted Flow Wind Tunnel of the University of Auckland . . . . . . . 35

3.3 Flat plate effective AoA under the influence of a sinusoidal oscillation followed by suddenly fixing the value of the angle of attack . . . . . . 46

3.4 Moth Foiler Wand Control System Explanation . . . . . . . . . . . 47

3.5 AC72 boat speed time history for the different numerical schemes . . 50

3.6 AC72 pitch and sinkage time history comparison using Euler and Midpoint methods . . . . . . . . . . . . . . . . . 51

3.7 AC72 boat speed time history comparison using Euler and Midpoint methods with different time steps . . . . . . . . . . 52

3.8 Moth sailing in foiling mode. Photograph by Manuel Ruiz de Elvira . 66

3.9 Moth polar curve generated using PASim. . . . . . . . . . . . . . 70

3.10 BSP time history of the Moth foiling transition. . . . . . . . . . . . 72

3.11 Sink time history of the Moth foiling transition. . . . . . . . . . . . . 72

4.1 Motion platform module block diagram . . . . . . . . . . . . . . . . 74

$4.249^{e r}$ dinghy sailing. Photograph by local Geelong yachtsman. Source: https://commons.wikimedia.org/wiki/File:49er_skiff_sailing_ AUS_nationals_Geelong.jpg . . . . . . . . . . . . 75

4.3 AC50 (a) and AC45 (b) sailing. Photographs by Manuel Ruiz de Elvira 76

4.4 6-SPS (a) and 6-UPS (b) platforms . . . . . . . . . . . . 81

4.5 Block diagram of the Inverse Kinematics implementation. . . . . . . . 83

4.6 Block diagram of the Inverse Dynamics implementation. . . . . . . . 86

4.7 Original flowchart of the Classical Washout implementation. Source: Reid et al., Figure 14.1 in $[151]$. . . . . . . . . . . . . . . . . 89 
4.8 Original flowchart of the Optimal Washout implementation. Source: Reid et al., Figure 14.2 in $[151]$. . . . . . . . . . . . . . .

4.9 Original flowchart of the Adaptive Washout implementation. Source: Reid et al., Figure 14.3 in $[151]$. . . . . . . . . . . . . . . . . . . . 93

5.1 VR engine module block diagram . . . . . . . . . . . . . . . 108

5.2 Beaufort 4 (a) and Beaufort 8 (b) sea states generated with Unigine . 112

5.3 Basic way to show data for training and designing purposes using Unigine114

5.4 Moth foiler Scaled model with V-SPARs in the Twisted Flow Wind Tunnel of the University of Auckland . . . . . . . . . . . . . . . . . . 116

5.5 V-SPARS processed image (a) and preliminary three-dimensional (b) of a V-SPARS analysis for the Moth mainsail in a twisted flow at 45 degrees of AWA . . . . . . . . . . . . . . . . . . . 117

5.6 Wool telltales . . . . . . . . . . . . . . . . . . . . . 119

6.1 Occulus Rift Touch hand-held controller. Photograph by Evan Amos. Source: https://upload.wikimedia.org/wikipedia/commons/d/d8/ Oculus-Rift-Touch-Controllers-Pair.jpg . . . . . . . . . . . . 124

6.2 Brushless electric motor with controller and integrated angular encoder 126

A.1 Time history comparison for different numerical schemes - BSP . . . 137

A.2 Time history comparison for different numerical schemes - Heel . . . . 138

A.3 Time history comparison for different numerical schemes - Pitch . . . 139

A.4 Time history comparison for different numerical schemes - Yaw . . . . 140

A.5 Time history comparison for different numerical schemes - Sinkage . . 141

A.6 Time history comparison for different numerical schemes - AWS . . . 142

A.7 Time history comparison for different numerical schemes - AWA . . . 143

A.8 Time history comparison using Euler and Midpoint methods with different time steps - BSP . . . . . . . . . . . . . . . 145

A.9 Time history comparison using Euler and Midpoint methods with different time steps - Heel . . . . . . . . . . . . . . . . . . . . 146

A.10 Time history comparison using Euler and Midpoint methods with different time steps - Pitch . . . . . . . . . . . . . . . . 147

A.11 Time history comparison using Euler and Midpoint methods with different time steps - Yaw . . . . . . . . . . . . . . . . . . . . . . 148

A.12 Time history comparison using Euler and Midpoint methods with different time steps - Sinkage . . . . . . . . . . . . . . . . . . . . . . 149 
A.13 Time history comparison using Euler and Midpoint methods with different time steps - AWS . . . . . . . . . . . . . . . 150

A.14 Time history comparison using Euler and Midpoint methods with different time steps - AWA . . . . . . . . . . . . . . . . 151

D.1 Setup used in the TWFT for Moth aerodynamic model measurements 170 


\section{List of Tables}

4.1 Cumulated linear sensed error for the Classical Washout . . . . . . . 90

4.2 Cumulated linear sensed error for the Adaptive Washout . . . . . . . 94

B.1 Time history of the simulated foiling Moth run using a heel PID controller 153

B.2 Detailed last 4 seconds of the simulated foiling moth run using a heel PID controller . . . . . . . . . . . . . . . . . . . . . 155

B.3 Time History of the Simulated Foiling Moth Transition Run . . . . . 159

B.4 Kinematic cues of the first four seconds of the Foiling Moth Transition Run Using Classical Washout . . . . . . . . . . . . . . . . 161

B.5 Kinematic cues of the first four seconds of the Foiling Moth Transition Run Using Adaptive Washout . . . . . . . . . . . . . . . . 163

C.1 Multi-threading tests time results using Moth Foiler models . . . . . 165

E.1 NSGAII algorithm results . . . . . . . . . . . . . . . . . 177

E.2 MOEA/D algorithm results . . . . . . . . . . . . . . . . . 178

E.3 NSGAIII (outer dimension reference point generation only) algorithm

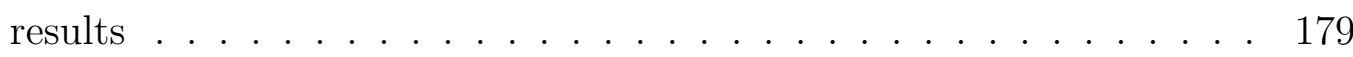

E.4 NSGAIII (inner and outer dimension reference point generation) algorithm results . . . . . . . . . . . . . . . . 180 


\section{Glossary}

The following glossary of the different sailing terms used throughout this work is provided for a better understanding of the text.

Angle of Attack (AoA): angle between a reference line of a body travelling through a fluid and the velocity vector of such a fluid.

Apparent Wind Angle (AWA): angle between the apparent wind vector and the boat centre line.

Apparent Wind Speed (AWS): magnitude of the apparent wind vector.

Apparent Wind: wind vector resulting from the combination of the true wind vector in which the boat is sailing and the negative of the boat speed vector.

Cant: angle of rotation on the local X-axis (approximately horizontal and longitudinal to the sailboat) of the different movable elements on-board.

Cunningham: downhaul found on Bermuda rigged sailboat attached to the tack of the mainsail.

Daggerboard: vertically moving vertical lifting surface used to generate the hydrodynamic lateral force to compensate the aerodynamic one.

Downhaul: trimming line allowing the application of a downward force on the luff of a sail.

Genoa: larger headsail that overlaps the mainsail.

Heel: angle of rotation on the X-axis of the sailboat.

Keel: fixed vertical lifting surface used to generate the hydrodynamic lateral force to compensate the aerodynamic one. 
Leech: the trailing edge of a sail.

Luff: the leading edge of a sail.

Mainsheet: trimming line attached to the boom principally allowing control of the mainsail position (in and out) by controlling the leech tension.

Offshore Racing Council (ORC): international body in charge of establishing and maintaining a series of competitive rules for offshore handicap sailing boat racing.

Outhaul: trimming line attached to the sail clew allowing for control of the shape of the foot of the sail.

Pitch: angle of rotation on the Y-axis of the sailboat.

Race Modelling Program (RMP): software that models the race, using VPP and weather data, between different contenders to estimate a win or loss probability for each one of them.

Rake: angle of rotation on the local Y-axis (approximately horizontal and transversal to the sailboat) of the different movable elements on-board.

Rudder: rotating vertical lifting surface normally located near the transom of the sailboat used for steering.

Toe: angle of rotation on the local Z-axis (approximately vertical to the sailboat) of the different movable elements on-board.

Traveller: trimming device normally consisting of a line and a track allowing the control of the boom position maintaining the mainsheet tension.

Velocity Made Good (VMG): speed of the sailing boat towards a certain target or destination (normally referred to a windward or leeward position and thus becoming the projection of the boat speed along the wind direction). Normally, it is different from the boat speed unless the boat is sailing directly towards such target or destination.

Yaw: angle of rotation on the Z-axis of the sailboat. 


\section{Chapter 1}

\section{Overview}

\subsection{Background and Context}

The America's Cup represents the pinnacle of sailing sport and has become one the principal exponents of how advanced technology and sports can work hand in hand. This assimilation has ensured that the competing boats of today are not simple sailboats anymore but complex machines with a huge number of setups and handling possibilities. Being able to understand the boat's behaviour and handling, and being able to find the best setup regarding the boat settings and the design parameters, lead the teams to achieve a better performance.

Until now, the main tool for the sail boat design team was the Velocity Prediction Program or VPP. This software is able to solve the boat equilibrium of forces up to the six degrees of freedom (DOF) of a rigid solid body. The information obtained after the equilibrium is solved (static approach) is the boat speed and the boat sailing attitude; the main elements characterising the boat performance. With the appearance of vertically lifting hydrofoils in the world of sailing, the need for new performance assessment tools became more and more obvious. The information generated by a traditional Velocity Prediction Program is no longer enough to understand the boat behaviour. Because of the dynamic nature of the foiling yacht, it is important to also know how the boat accelerates, the stability and how the boat handles. A new approach has appeared allowing us to obtain all the information needed. Instead of facing the problem as a static one based on the force and moments equilibrium, this new approach consists of solving the equations of the dynamics of the rigid body in the time domain. This is the reason for the appearance of the dynamic Velocity Prediction Program (VPP). The main problem now is to find a way to be able to interpret all this information in the manner that allows the designer and the user to understand how the boat behaves, and then apply all that knowledge to improve the 
boat design and the crew boat-handling abilities. New forms of representation and interaction are needed and a new layer of complexity has to be implemented over the dynamic VPP leading to the development of the physical sailing boat simulator.

\subsection{The Simulator: A Definition}

According to Manuel Ruiz de Elvira's PhD Thesis [156] the simulator must provide enough information for the user, the sailor, to generate the best user experience, as close as possible to the reality. The simulator has to be able to also accept inputs from the user in the same way a sailor interacts with an actual boat. In summary, it is a two-way relationship that allows the user to feel he is actually sailing a real sailboat. To be able to achieve that, some of the sailor's senses must be stimulated in the same way they would be on a real boat. For instance, the simulator has to reproduce, allow or provide:

- The boat accelerations.

- The visual surroundings of the moving boat.

- Any data available on-board about the external conditions affecting the boat, including wind parameters, VMG...

- The way to interact with the boat elements such as rudder tiller, sail trimming controls...

The main objective when developing such a tool is to find the way to accomplish these requirements. From the technical point of view, the simulator is then the sum of a number of elements working together to produce the result needed. For instance:

- A time-domain dynamic VPP, which brings together all the models describing the physical reality of the boat and capable of calculating the yacht behaviour based on different environmental and user inputs.

- A parallel robot or moving platform being able to reproduce the boat accelerations.

- A Virtual Reality (VR) interface like a VR headset to reproduce, in an immersive way, all the visual environment related to a moving yacht.

- A physical user interface that allows the sailor to handle and adjust the virtual sailboat the same way as he usually would in a real one. 
These four elements represent the essential ones that the simulator needs to give the best experience. Without them it is not possible to offer a virtual sailing experience as close as possible to the reality, knowing that a perfect match in the user sensations is impossible. Other elements could also be integrated such as a Race Modelling Program (RMP) to model actual racing situations with other competitors. The goal is then to have an experience that could help the sailing team and the design team to improve their handling skills and the overall performance of the boat.

\subsection{State-of-the-Art}

In such a competitive world, any advantage can be the key to beat your rival so having the access to such a tool can make all the difference. Surprisingly, any of the attempts to develop such tools were partially unsuccessful in the past. All the efforts seemed to head in a different direction, such as, a tool to teach how to sail or to evaluate physical performance of the crew.

The first attempts to simulate a sailing boat were carried out in 1965 by Hansen [73] with his "Sailing Simulator" patent, later Waddington et al. [181] in 1971 presented their "Simulating apparatus for teaching the art of sailing" and in 1976 Nishimura and Kaoru [123] also presented their patent of a "Sailing Simulator".

In the late 1980's and early 1990's two simulators were developed by Harrison ([24], [111]) and Blackburn ([16], [17]) to study the physiology of hiking in a dinghy in a controlled environment. Harrison's simulator consisted of a rotating, one degree of freedom, Europe dinghy deck molding, moved with a weight system and using pumped water to simulate the heeling moment, and completed with a primitive graphical and control interface. Blackburn's simulator was composed of an Olympic Laser Class dinghy mounted on a fixed frame to allow them to measure the hiking moment. A video of actual Laser sailing was projected using a television screen to allow the sailor to mimic the movements on the video being shown. This concept of using the simulator as a laboratory tool was used more recently by Cunningham and Hale in 2007 [37].

With the efforts heading in other directions, in 1994, Kibuchi et al.[93] developed a sail training simulator focused on the teaching of sailing instead of physiological research. It was formed by using a single-handed sailboat with pneumatic rams to control the boat's roll motion and the boom and rudder moment feedback. It was completed by two computer graphics displays, one in the bow and another in the 
stern. To reproduce the manoeuvring characteristics of the boat, a mathematical model was implemented.

Based on Harrison's simulator, in 1994 Walls and Saunders [184] developed an Olympic Laser class dinghy simulator. It consisted of a deck molding of the boat supported by a steel frame, using a computer-controlled weight system to reproduce the heeling moment. The device was completed by a trimming and steering mechanism and a computer display. In order to obtain a more realistic experience, Gale and Walls improved this design incorporating new elements as is presented in [62]. Such elements were a mathematical model of the boat dynamics, a new motion control system based on pneumatic rams and an improved 3D graphics visual system with audio feedback. The dinghy deck was mounted on a pivoting support frame and had the ability to be changed in order to simulate different classes of boats. The boat motion was driven by a pneumatic ram counteracting the righting moment of the hiking sailor. Another pneumatic ram was connected to the rudder tiller to simulate the rudder moment. It was completed by a spring system reproducing the mainsheet tension. To improve the sensation of reality, some elements of the deck gear were positioned in their real position (original mainsheet blocks and tiller). Finally, the 3D graphics visual system was able to reproduce different elements such as hull, rig and water, including shading for the wind gusts, and different floating elements. The main goal of all this work as described in [62] was to obtain a simulator that in comparison to a real dinghy "should have had similar appearance and dynamic response, elicit similar posture, body movement and decision making processes from the sailor, give appropriate sensory feedback and provide appropriate decision making cues". The increased capabilities of this simulator allowed Walls et al. [183] to carry-out the performance assessment of different sailors in upwind dinghy-sailing conditions.

All this effort made by Gale and Walls lead to the development of the first commercial sailing boat simulator, the Virtual Sailing VS-1 described by Binns, Bethwaite and Saunders in [12]. Some changes were made over the original simulator; for instance, a full dinghy suspended on rollers was used to obtain a more realistic feel of the boat and the roll angle. A new pneumatic system was adopted with higher pneumatic capabilities allowing a faster and more powerful response. The physical model of the boat was also changed, this time using some basic VPP or velocity prediction program techniques and new physical models. The original system based on three degrees of freedom (forward force balance, heel moment balance and yaw moment balance) was replaced with a four degrees of freedom model, adding side force balance. 
Since then, the simulators created by Virtual Sailing were used in different areas. In [115] Mooney et al. present several different uses:

- Olympic sail training.

- Engineering education.

- Disabled sail training.

- Instruction for novice sailors.

In 2012, a Simulation Verification, Validation and Calibration (VVC) of the Virtual Sailing simulators was conducted by Binns et al. [13]. On-water measurements of different manoeuvres were taken with a specially developed data acquisition system. The same procedure was made with the simulator, repeating the same manoeuvres and collecting the same data. All the data was then compared to show a good correlation between sailing times measured on-water and those obtained with the simulator. Some problems regarding the fidelity of the rudder force feedback system appeared.

Further work regarding the effect of motion on presence for a sailing simulator was performed by Mulder and Verlinden in [117] and [116]. They developed a system with a dinghy hull mounted on top of a Stewart platform ${ }^{1}$ with the visualisation system mounted outside the platform. The goal was to evaluate the feedback of five sailors to roll, pitch and heave displacement during the simulation with the possibility of adjusting the parameters controlling the amount of movement in different runs. The results showed that the most engaging motion to contribute the sense of presence was the roll motion. Pitch motion was considered of less importance except when combined with roll motion, improving the sense of presence. The authors consider that heave motion can almost be negated, showing that for advanced athletes it is more important to use detailed graphics with a large field of view. Based on the past experience, the same authors developed a motion system for a sailing simulator in [116] focusing only on roll movement and mainsheet and rudder advanced force feedback. One more layer is added in [180] with the study of the effect in presence of using an array of fans simulating the wind. The results were promising but showed that the users focused more on sound than airflow necessitating the importance of obtaining a powerful and silent wind system being able to reproduce proper wind temperature.

\footnotetext{
${ }^{1}$ The Stewart platform or Stewart-Gough platform is a 6-DOF parallel robot used for motion reproduction and spatial positioning.
} 
Following a different path than the one presented in [117] and [116], Avizzano et al. [5] created a sailing simulator with a Stewart platform on top of which they built a yacht cockpit mock-up and a visual system. Instead of reproducing the actual motion, they chose to reproduce boat accelerations filtered by a washout algorithm as applied in the commercial aircraft simulators.

Other works based only on the numerical simulations, VPP's and RMP were developed to study different aspects of sailing. For example, Masuyama et al. [103] developed a tacking simulation in order to be able to obtain the best tacking procedure, or the work of Lidtke et al. [99] with an America's Cup 45 yacht tacking simulator which includes some sort of physical interface (mock-up of the deck). Keuning et al. [90] made a similar work but with a different objective, in this case with handicapping assessment purposes. Philpott et al. [140] and later, Scarponi et al. [158] showed the advantage of using such tools in yacht performance optimization and analysis. Some numerical simulators were developed to improve the knowledge and training of specific parts of a regatta, such as the work by Binns et al. [14] regarding starting manoeuvre training with an America's Cup yacht.

\subsection{Contributions}

The main contributions of this work are to analyse the potential uses of such a tool, to present a general and modular framework that will aim to demonstrate a fully immersive, simulated foiling sailing boat experience and how to integrate the different systems of the simulator. This is the result of the research and development of different interfaces and elements, such as:

- The physical models and autopilots needed by the dynamic VPP to calculate and reproduce, in the most accurate way, the boat behaviour. These range from simple models to complex sets of interpolated data. The main challenge presented here is being able to obtain the right combination of speed and accuracy during the six degrees of freedom dynamic calculation that will be the core of all the different systems presented in the simulator.

- The interface that will drive a physical six degrees of freedom platform and will manage the acceleration output of the VPP. This output will be filtered using a washout algorithm with coefficients obtained by using multi-objective optimization evolutionary algorithms. 
- The virtual reality interface that was developed to face the needs of the user and the different options considered. New visualisation technologies appeared recently will be analysed.

- The interface allowing the interaction between the user and the simulator to produce the correct input to control the simulator.

As outlined in 1.3, other attempts have been made in the past to achieve a similar outcome. However, instead of using a restricted approach with 3 or 4 degrees of freedom (which previously worked well with conventional sailing boats), this thesis will implement a full 6 degrees of freedom dynamic approach as an answer to the needs of the new generation foiling boats.

\subsection{Outline of the Thesis}

In order to answer all the stated goals, this thesis will be organised as follows:

- Chapter 2, The Sailing Yacht Simulator: In this chapter, the concept of a sailing yacht simulator will be discussed. The possible uses will be explored and some concepts and remarks will be presented.

- Chapter 3, PASim, The Simulator Core: The first and the most important of the components of the boat will be presented and analysed. The VPP is the core of the simulator and will be responsible for calculating and providing all the data regarding the behaviour of the boat. The principles of the dynamic calculation, the physical models, the autopilots, etc, will be presented in this chapter.

- Chapter 4, The Physical Platform: The interface in charge of transmitting to the user the sensations related to the boat accelerations is presented. The physical platform is presented along with the methodology to calculate the way it moves after receiving the acceleration input from the VPP. Because of the limitations of the platform, the boat acceleration calculated by the simulator core has to be filtered by a washout algorithm. This algorithm is presented along with a training procedure to obtain the filter coefficients using real sailing data and Multi-objective Evolutionary Algorithms. 
- Chapter 5, Virtual Reality Interface: In this chapter, the interface in charge of representing the visual environment related to a moving yacht is analysed. Different options are presented in response to the needs of the simulator, ranging from the code in charge of the representation (Virtual Reality (VR) engine) to the elements (Display, VR headset) that will represent the moving boat.

- Chapter 6, The Physical User Interface: The last component of the simulator will be the interface allowing a certain amount of user interaction with the simulator. Here three elements will be analysed. The first one in charge of the steering inputs, the second one in charge of the sails control inputs and the last one in charge of the determination of the user position.

- Chapter 7, Conclusions: Finally, in the last chapter, the main conclusions obtained from this work will be drawn. 


\section{Chapter 2}

\section{The Sailing Yacht Simulator}

The concept of a sailing boat simulator has to answer all the potential problems that a competitively high-performance sailing team could face. For instance, it has to be:

- The base element for the design team: the principal design asset in the decisionmaking process.

- A tool that will improve and facilitate the process of testing from a design solution to a handling strategy.

- A tool that will improve communication and the understanding between the different departments composing a high-performance sailing team (such as sailors, designers, performance analysts and coaches).

- A tool that has to be useful for the sailors' training, assisting their understanding of the boat leading to an improvement of their skills.

The main objective, as with the tools used before, is to be able to present the highest performance of the boat and the crew together across the competition. The simulator can be used to address each of the four ideas just described and present a whole new world of benefits away from the traditional uses of the current simulators in the market ([115]). Every individual or integrated component that combine to form the simulator will answer specific needs and help to address specific problems. All the benefits of such a tool will be presented in this chapter. 


\subsection{A New Design Asset}

\subsubsection{Advanced Performance Assessment}

The simulator is formed by different tools that can help the performance assessment. When the boat performance is known from on-the-water testing, using an actual full dynamic simulation of the boat, complete with other analysis tools, could help to obtain the information needed to determine the explanation on why the actual performance differs from the expected one.

In advanced stages of the design process, when the boat is already built and the athletes are actually training on board making fine adjustments to all the equipment, having such information could help and speed up the process of analysing the data generated while sailing.

If the situations and settings that could degrade the boat performance are identified while using the dynamic simulation, a system analysing all the telemetry data on-board could identify and generate warnings in real time helping the sailors to avoid poor performance situations in advance, maximizing the overall performance of the boat and therefore serving as a performance target advisor for the sailors.

While comparing real data and virtual data, some level of uncertainty is expected to come from the real world. To have a more realistic base for comparison between both sets of data, it is possible to add some signal noise to the latter. Since normally the probability density function of the noise is unknown, a comparison between onboard measured data and simulated data in the same conditions can be used to establish an approximate noise distribution, although this is not a trivial task. This process can be done with every measured variable and then taken into account while running the full simulation routine, potentially obtaining a more realistic environment.

\subsubsection{Early Testing of Design Features}

If the correct physical models are implemented in the dynamic VPP, the sailing simulator can be used as the main decision-making tool in early stages of the design. This will speed up and assist design decision processes without having to wait for construction time or on the water testing.

This aspect of the simulator is not only limited to the design evaluation of different alternatives from the performance perspective. It could also be used as the platform that will allow evaluation of the actual feasibility and on-board use of different design 
alternatives. This will avoid possible mistakes when expecting a certain performance from certain solutions that cannot be realized while sailing the boat.

\subsubsection{Assistance to Energy Management}

As previously discussed, sometimes a good design solution presents a good performance but could be infeasible on board. As observed in the 35th America's Cup, the main problem with cutting-edge extreme performance technology, like unstable foiling platforms, is the huge consumption of energy that drives their control systems. Normally, sailing being a sport, all the energy has to be generated on board by the crew and normally is limited in storage and in time. The amount of energy on board and how it is used while sailing could be critical and can dictate the only way to perform a manoeuvre. The energy management could transform very good design solutions into very inefficient ones if the energy is not handled well. Normally, you have to wait to have the yacht built with all the systems to test if a solution could be good from the energy point of view. With the simulator, this could be done before the system is even built. With the right models implemented it is possible to do this in a deterministic way allowing for the establishment of the energetic viability of a certain solution or even to determine the best strategy for an energy efficient adjustment while keeping the performance in mind.

\subsubsection{Continuous Dynamic VPP Verification}

One of the advantages of the simulator is the ability to make a subjective evaluation of the dynamic VPP results outside the usual process of numerical verification. Sometimes, it is difficult to identify strange patterns and behaviours by only looking at the raw dynamic data. In some situations, even using specific data representation is not enough. Being able to actually sail a virtual boat could give subjective information about the quality of the physical models implemented and their validity.

\subsubsection{Two Boat Performance Evaluation with Only One Real Boat}

A two-boat campaign usually allows on-the-water performance comparisons of different elements or settings. Sometimes, the rules forbid this kind of campaign, allowing only the construction of one boat. In such situations, the second boat could be substituted by a virtual boat in the simulator, sailing with the same weather data collected by the real boat. In fact, the use of a virtual boat will speed up the tests and allow 
swapping of different configurations easily without having to make any change on a real boat.

\subsection{The Perfect Testing Platform}

\subsubsection{No Dependency on Weather Conditions}

As introduced before, one of the main problems when planning a series of tests onthe-water is the lack of control over the main conditions that affect the behaviour of a sailing boat. These conditions are mainly related to the weather and can be predicted but not controlled. Inside the simulator, these weather conditions could be arbitrarily set, making the test independent from the weather and therefore highly productive.

\subsubsection{Evaluate Safety Limits and Corrections}

To address the limitation of the lack of movement range from the physical platform, the inputs are normally filtered using a washout algorithm which will be explained in the following chapters. Extreme situations, like capsizing, will not be fully reproduced physically, but this setback could be transformed into an advantage. The simulator allows reaching such limits, knowing that you are approaching them, without risking the crew or the boat integrity. This is the perfect tool to evaluate possible reactions, settings and handling corrections to avoid dangerous situations before reaching the point of no return. It can also be used to help inform sailors' perceptions when reaching the boat limits.

\subsubsection{Evaluation of Optimal Response to Soft Transitions and Patterns of Changes}

Even if a dynamic VPP could be used to analyse and determine what is the optimal procedure to manage transitions while sailing (tacking, gybing, facing a wind gust or a wind shadow, etc.) through a sequence of actions and adjustments, those need to be executed within the capabilities and reaction time of the crew sailing the boat. The simulator offers a sufficiently realistic environment to test and validate strategies and crew suggestions, or to let the sailor reach the best solution, following his intuition. Such tests can be evaluated and analysed by the design team in real-time, offering almost immediate feedback to the simulator user without waiting to be back on shore to analyse on-the-water data. This testing could be extended to the training of the 
interaction between every member of the crew, helping to determine which ways of communication are best to achieve the highest performance in those transitions. In the majority of cases, manoeuvres where interaction between crew members is needed can be trained and assessed.

\subsubsection{Evaluation of Situations with Similar Performances but Different Executions}

Sometimes, two different sailing procedures may lead to similar yacht performance. These procedures consist of a combination of adjustments, trimming settings and steering changes following a certain order. These days, in some competitions, the training time is limited by the competition rule so it has to be as efficient as possible. The simulator provides the possibility to try such procedures in a safe environment and in realistic conditions without compromising the security and integrity of the boat. The sailors will then feel more comfortable and open to test further options and select the actions sequences that will give them the most confidence. This pre-acquired confidence can maximize the chances to sail the yacht at maximum performance even in on-the-water training and testing. Another important feature is related to energy management, specifically the ability to check if such procedures are compatible with the amount of energy stored on board.

\subsubsection{Evaluate Early Signs for Required Corrections}

While sailing the boat, a huge amount of trimming and setting corrections are made by the crew when they perceive changes in the conditions affecting the boat. Sometimes, these changes, which need time and energy to be completed, could be anticipated by the observation of early signs of performance degradation. A proper simulation tool can help to identify such early signs and later create on-board auto-pilots or, if not allowed, careful instructions on how to anticipate and how to address such corrections. The sailors will be able to anticipate their actions to produce the best performances or optimize the energy consumption process allowing for energy regeneration on-board in readiness to face other race situations.

\subsubsection{Test Deck Gear}

The actual process of designing a deck layout consists of making a number of decisions and assumptions that later will be confirmed, or not, once the real one is built. If the project is big enough, a mock-up of the deck can be built on a one-to-one 
scale. Thanks to the mock-up and through a process of trial, error and discussion between the sailors and the designers, a lot of different ergonomic aspects can be addressed. When building a mock-up is not possible, the changes to improve comfort and ergonomics are made within the real boat when tested on-the-water. These changes, once the boat is already built, are more expensive in terms of money and time, but give the real answers to the problems directly related to kinetic and kinematic effects. Until now, the best approach was to use the mock-up to make the most important decisions and then fine tune such decisions with the experience acquired with on-the-water testing.

With the simulator, the testing time with the actual boat could be reduced significantly or even completely eliminated using the mock-up mounted over the physical platform to reproduce the actual motion or accelerations of the boat. This allows the user to optimize the efficiency of the deck layout or even the selection of the proper gear in a more realistic situation without having to build the boat or wait for the optimal weather conditions to carry out a real test in a safe environment.

\subsubsection{Provide a Test Platform for Augmented Reality}

A key aspect of sailing is the way information is given to the sailors when they are sailing the boat. This is even more important in high-performance sailing where every piece of information, allowed by the competition rules, can make a difference in performance. Since the first introduction of navigation instruments and on-board electronics, used to provide essential data such as the apparent wind speed (AWS), apparent wind angle (AWA) and boat speed (BSP), the amount of measured data available has increased drastically.

This amount of information could be overwhelming since the sailor has to deal with his own perceptions and sensations, communications and all the data available and processed in real time on-board. All these elements can interfere with each other and this may alter the sailors' capacity to make the right decisions. This could be solved using augmented reality, personalising the information and communication interface for each member of the crew. The amount of personalisation is vital because every person needs a different kind of stimuli and different kind of information. To be able to answer all the needs of each crew, the person in charge of designing an augmented reality interface has to test a huge amount of different combinations and possibilities. These tests will give the design team the correct way to select and supply the right information, using the right channel, at the right moment and with minimal disruption to other tasks the crew has to perform. The main challenge is to 
emulate the real situation on-board when on-the-water testing is not a viable option. A more flexible platform could be used to explore and evaluate specific gestures or actions from the crew when using the augmented reality interface to face different racing situations. Such triggers could change the information displayed and adjust it to improve tactics when doing a pre-start sequence, or crossing the starting line, etc.

In this case, the full sailing simulator could be used to accomplish these tasks, allowing the design team to directly measure the effect of the supplied information and how it is delivered to the crew regarding the yacht performance.

\subsection{A New Interface for Improved Communica- tions Between Athletes and Designers}

One of the main benefits of using a simulator in a high-performance sailing team is that it can be used as an effective tool to help the sailors and the designers to understand each other.

When talking to the athletes, the design team has to relay any kind of knowledge or technical information in a way that is understood by everybody. Even with highly technical crews, the sensations for the athletes when sailing remains an important assessment tool. When the performance of the boat depends on sailing a boat in a way the crew considers unusual or not normal, the design team could, by using the simulator, show how the boat could perform when such way is used.

In the opposite way, sometimes when a sailor tries to explain how it feels sailing the boat, it is hard to really express every sensation in a technically articulate manner.

In early 2000, with the America's Cup class monohulls, this was done during sea trials with members of the design team on board. Unfortunately, with foiling, the boats are weight and space sensitive making them more dangerous and sea trials less viable due to the risk involved. The simulator can replace this on-board shared time between the design team and the sailor with a new common ground where both of them can evaluate and discuss, quickly and safely, different solutions regarding design decisions or yacht handling. This ideal environment increases the opportunities to share information with each other, multiplying the number of new ideas and making the design evolution easier. 


\subsection{A Sailor's Training Tool}

\subsubsection{Assistance Towards a Steeper Learning Curve}

From the sailor's perspective, when the first boat is built it is always hard to be completely confident with the use of the boat. This problem is even more significant when competition rules force the teams to limit their training time on the water or when using the new technology on advanced and difficult-to-sail foiling boats. With limited training time, the pressure on the sailor in relation to learning how to handle and control the boat is immense. The on-the-water training has to be prepared in advance in order to minimize or even avoid mistakes, which can lead to big setbacks during the design, training and testing process.

With the ability to reproduce the behaviour of the yacht, the simulator is able to give a proper sense of how the boat is sailed, with the big advantage of being in a safe and controlled environment. This is the reason why, when the sailing team uses the simulator they are able to obtain immediate feedback and evaluate how their actions on board affect the boat behaviour and performance. The simulator then facilitates the understanding of the yacht and consequently provides assistance towards the steeper learning curve that actual foiling boats present. This is translated into a higher quality of on-the-water testing and training time with a huge improvement in the overall productivity.

\subsubsection{Training for Subsequent On-The-Water Tests}

One of the main parts of a high-level sailing campaign is the on-the-water tests. They are always very time consuming and the data extracted from them can be difficult to interpret. Sometimes, the actions required for a specific test are difficult to perform correctly in real time without the proper crew preparation.

The simulator can help to practice those tests and give an idea about what kind of results will be expected or if it is even possible to make them by the crew. With all this information and with the sailors ready to perform such tasks, the number of final tests can be reduced avoiding repetitions or even eliminating useless or inconclusive tests before even touching the water. This allows improvements to the test schedule in advance and improved use of the time spent on the water. 


\subsubsection{Race Preparation}

During an international competition such as the America's Cup, teams from all across the world compete in a unique and specific location. Sometimes the teams can choose to develop their boat far from this location in their home bases. This could have some advantages, mainly related to the comfort of working in a known place and sailing in a known venue. From the technical point of view, this can also present an advantage, specifically privacy regarding development which would hinder and discourage espionage attempts by rival teams.

The main disadvantage is that sailing in a different venue than the one to be used during the competition, means sailing in a different environment with different points of reference and essentially different weather, sea and wind patterns. Normally the teams have to make a trade-off, so the early stage development and training is made in the home base and then the whole team moves to the actual competition venue.

With the use of a simulator, if the environmental patterns are properly identified and modelled, then it is possible to train the crew in the same conditions that will be expected during the competition but without the need to move to the actual regatta venue. A clear example of this situation is the strategy followed by Emirates Team New Zealand during the 35th America's Cup in 2017 in Bermuda, training in their home base until just several weeks before the competition and being able to win the Cup.

\subsubsection{Model Racing Situations}

Following the same idea presented in the previous section, with the simulator it is not only the venue characteristics which could be reproduced. With proper racing data, using a race modelling program (RMP) or simply simulating two boats with two crews controlling the simulator, it is possible to reproduce and then evaluate specific racing situations, from wind changes to actual interactions with the opponent boat.

As previously stated, such interactions could be modelled by means of different elements. To test realistic tactical situations where the decision-making process is vital, the RMP could model the opponent behaviour under varying wind conditions or configurations. If the RMP modelling is not enough to simulate specific situations, two boats competing against each other could be used within a single simulation, running one instance of the dynamic VPP for each boat and providing visual feedback related to the opponent yacht's behaviour back to the VR environment. 


\subsubsection{Training of Natural Reactions}

During an actual racing situation, external conditions may change affecting the boat behaviour. The sailors have to perceive these changes and act as quickly as possible regarding boat handling and settings to maintain the best performance. Minimizing this reaction time is vital in order to reduce the performance loss.

Using the simulator, such changes can be consistently repeated and the effect of reactions of the crew evaluated. After some repetitions and using a trial and error strategy, some reactions can be trained in order to favour some behaviours in the crew achieving the best performance but maintaining a reasonable amount of safety. It is possible then, to train and improve responses from the crew to trim to target directives using the simulator capability of implementing autopilots. Even if some part of this training could be done using only the virtual environment (mainly visual stimuli), with the usage of a physical platform to reproduce boat accelerations the inertial effects in manoeuvres on the crew could be included.

\subsubsection{Training Anticipation Regarding what to Expect from Unusual Situations}

High-level sailing is a sport where every mistake or bad reaction to an unusual situation can lead to an obvious disadvantage. When this happens, the opponent will use this to his advantage to win. It is therefore important to be able to work on minimizing that risk of being exposed to a disadvantageous situation. Within the simulator, such unusual situations can be created to evaluate and train the sailor's reaction without putting the boat or the crew in a dangerous situation, making this tool ideal for the evaluation of "what if?" scenarios.

\subsubsection{Anticipate the Behaviour of a New Design or Type of Yacht}

From the point of view of a sailor, a new type of yacht or even a new concept could be a challenge, taking him away from his usual comfort zone. Once the boat is built, reeducating all his natural reactions while being on board could be time-consuming and affects the final training and on-the-water testing. A realistic simulator can provide a huge amount of information that can help sailors to understand how the boat will behave when they are not familiar with it. It could even be used as an early stage educational tool to train and monitor perceptions and reactions of the sailor. 


\subsubsection{Virtual Repetition of Real On-The-Water Training and Regattas}

After training on the water or after a regatta, in high-level events, a briefing is held between the crew and the coaches to analyse the training session and the quality of manoeuvres using the video recorded by the on-board chase boat cameras. If the boat and wind data are recorded, the training session can be reproduced inside the simulator. This methodology presents some advantages over the video-only briefing. It allows to repeat and train specific parts and moments of the sessions, handling the boat if necessary, to improve and learn how to avoid or correct mistakes and poorperformance situations. It also allows the coaches to better understand the boat behaviour and crew feedback, improving future training planning.

\subsection{A Modular Approach of the Sailing Yacht Sim- ulator}

As previously explained the sailing yacht simulator can be used to fulfil a wide range of the design and sailing team needs. It is important to point out that the simulator components may be different for each of the different uses. The simulator has to be designed as a flexible tool that can be adapted fast and easily to the technical needs associated with each of the design and training stages. The software side has to be modular, allowing to increase the level of complexity and the elements and cues reproduced. For instance, the same simulator software will be used:

- For design purposes and basic boat behaviour exploration:

- The simulator has to be able to run on one machine using a simple visualization system, such as a conventional screen or projector.

- It uses the time-domain VPP modules and sub-modules (including autopilots) and the virtual reality (VR) engine to handle the visual part.

- For design purposes, the VR engine can be switched off. It is not always necessary and data can be analysed using simple charts and plots.

- For advanced design purposes and basic training:

- The simulator has to be able to run on one machine, using a simple visualization system or a VR headset for training. 
- It uses the time-domain VPP module and sub-modules, the VR engine (with the possibility of using a VR headset) and the user operation input modules allowing boat handling and settings adjustment.

- For advanced training and extended boat behaviour analysis:

- The simulator has to be able to run on one or multiple machines using a complex visualization system (multi-screen configuration or VR headset), with the possibility of running the motion platform system and implementing advanced operation interfaces.

- It can use all the modules of the simulator: the time-domain VPP, the VR engine, the motion algorithm and the operation interface input module.

- For improved performance, the main modules can be run in independent machines communicating between each other using efficient network hardware and protocols.

Other configurations are possible, activating or not any of the modules (except the time-domain VPP, which is the core of the simulator). All the items cited previously will be described and analysed all along this thesis. A general diagram of the modular implementation of the different modules of the simulator is presented in Figure 2.1. 


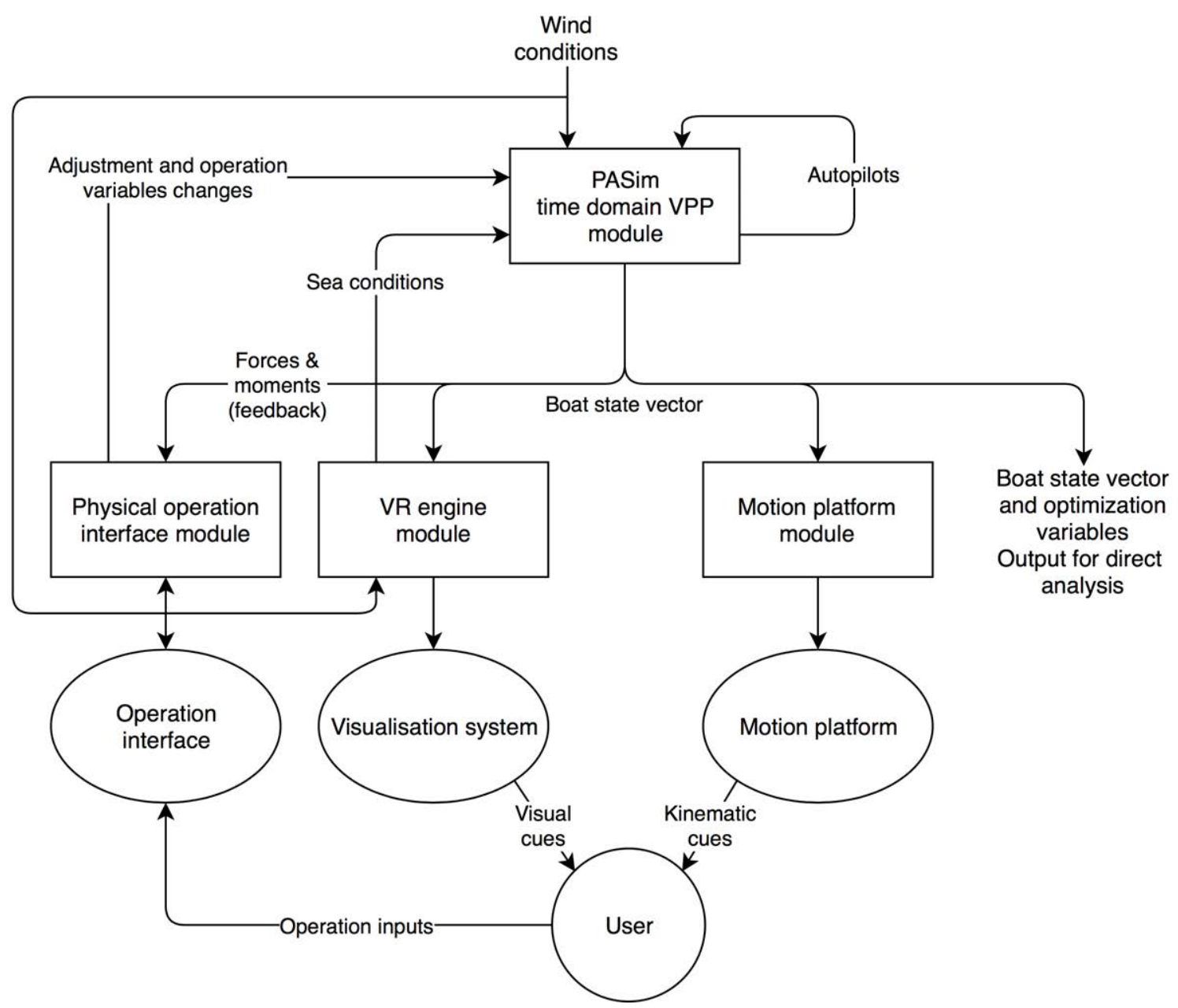

Figure 2.1: Simulator general diagram of the modular implementation.

\subsection{The Concepts of Immersion, Presence and Fi- delity}

In order to evaluate and describe the different elements of the simulator three specific concepts will be used:

- Immersion

- As explained in Cummings et al. in [36], Immersion "can be regarded as a quality of the system's technology, an objective measure of the extent to which the system presents a vivid virtual environment while shutting out physical reality". From the simulator point of view, it will be related to the capabilities of the different systems to shut out user from the reality into a vivid sailing experience. 
- Presence

- Presence can be defined in different ways as presented by Lombard et al. in [100]. Within this paper, the definition that suits better the thesis context is the one that defines presence as the idea of "perceptual and psychological immersion". The perceptual immersion is described by Biocca et al. in [15] as "the degree to which a virtual environment submerges the perceptual system of the user", in other words, the degree to which the simulator is able to replace the physical environment sensed inputs with the ones generated by the different simulator systems. The psychological immersion is related to how the user feels and reacts to immersion ([100]).

- Fidelity

- Fidelity is defined by Rehmann in [149] as "a function of the degree to which the equipment and environmental cues relate of the real airplane", in the context of this thesis it is how they relate to the ones of the real sailing boat. Fidelity can be objective (measurable differences between simulator generated and real cues) or perceptual (user perceived differences between simulator generated and real cues) as explained in the AGARD Advisory Report No. 159 [91]. The lower the fidelity, the bigger the differences between simulator generated cues and real sailing boat ones are and vice versa. 


\section{Chapter 3}

\section{PASim, the Simulator Core}

\subsection{The VPP as the Heart of a 6-DOF Sailing Sim- ulator}

The main objective when developing a simulator is to find a way to produce a virtual reality as close as possible to actual reality. In the case of the sailing boat simulator, the goal is to be able to reproduce virtually the behaviour of the boat under certain circumstances. In other words, the key is to be able to mimic the boat using models of the physics involved in the act of sailing. Such models have to represent the forces and moments acting on the different elements of the boat. Different levels of reality could be achieved depending on how many degrees of freedom (DOF) you can reproduce. The DOF refers to each of the three linear motions and the three rotations that the boat can make regarding the system of reference used and they are a consequence of the three resultant forces (Fx, Fy, Fz) and three resultant moments (Mx, My, Mz) applied to the boat. For example, for a conventional, slow monohull, it could be enough to reproduce 4 degrees of freedom (X-motion, heel, pitch and yaw rotations) to achieve a good amount of reality. However, to reproduce the true nature of foiling boats, six degrees of freedom have to be used.

Since the beginning of yacht design, the need for a powerful performance prediction was paramount. At the beginning of the twentieth century, yacht design was mostly based on pure observation and trial-and-error. Such techniques gave birth to incredible machines, such as the J-Class boats. The improvement of the physical knowledge and technology proved that a specific performance assessment tool was needed. The first serious attempt to develop a specific VPP tool was presented by Justin E. Kerwin in his 1976 paper "A Velocity Prediction Program for Ocean Racing Yachts" [87]. With two degrees of freedom, it was able to solve the forces and 
moments equilibrium for the hull resistance with the sail-plan driving force and for the sail-plan heeling moment with the hull stability. This work was embedded in a combined project by the Offshore Committee and US Sailing to create a new handicap system to replace International Offshore Rule. The new Measurement Handicap Rule (MHS) was then developed within Boston MIT under the supervision of Professors Kerwin and Newman [88]. This proposal was the seed of the actual International Measurement System (IMS) measurement system and its more refined VPP tool [33].

Even for the slower conventional monohulls, the sailing boat attitude and speed obtained using VPP equilibrium calculation could be enough from a performance prediction point of view. For fast foiling boats, knowing the boat dynamics and all the available data regarding multiple DOF stability is paramount to making a proper assessment of the boat performance.

For a general overview of PASim implementation within the simulator please refer to the diagram in Figure 2.1.

\subsection{From a Static Equilibrium to a Time-Domain Calculation}

As explained by M. Ruiz de Elvira all across his PhD thesis [156], the general 6-DOF general approach used in PASim is the most adaptable way to evaluate performance in a sailing yacht. With the ability to select the number of degrees of freedom, every type of boat calculation could be done: from simple situations to more complex configurations. For each calculation, the problem consists of solving the system of non-linear equations presented below in equation 3.1 (with up to the six equations presented or less depending on the number of DOF selected) using simple numerical schemes like the Newton-Raphson or more complex non-linear optimization solvers. The nomenclature used in 3.1 is the same as the one used by Ruiz de Elvira in [156]:

- $\mathrm{F}$ for forces.

- $\mathrm{M}$ for moments.

- S sub-index for static (For models related to gravitational and hydrostatic effects).

- D sub-index for dynamic (For models depending on the incoming flow velocity).

- The usual spacial variables $\mathrm{X}, \mathrm{Y}, \mathrm{Z}$. 
- $\phi$ for heel angle, $\theta$ for pitch angle and $\varphi$ for yaw angle.

- $V_{S}$ for the boat speed.

- $\delta_{S}$ for the steering parameter (rudder angle or others acting on the yaw moment).

- $Z_{S K}$ for the boat sinkage.

$$
\left\{\begin{array}{l}
\sum F_{X D}\left(V_{S}, Z_{S K}, \phi, \theta, \varphi, \delta_{S}\right)=0 \\
\sum M_{X D}\left(V_{S}, Z_{S K}, \phi, \theta, \varphi, \delta_{S}\right)+\sum M_{X S}\left(V_{S}, Z_{S K}, \phi, \theta\right)=0 \\
\sum F_{Y D}\left(V_{S}, Z_{S K}, \phi, \theta, \varphi, \delta_{S}\right)=0 \\
\sum M_{Y D}\left(V_{S}, Z_{S K}, \phi, \theta, \varphi, \delta_{S}\right)+\sum M_{Y S}\left(V_{S}, Z_{S K}, \phi, \theta\right)=0 \\
\sum F_{Z D}\left(V_{S}, Z_{S K}, \phi, \theta, \varphi, \delta_{S}\right)+\sum F_{Z S}\left(V_{S}, Z_{S K}, \phi, \theta\right)=0 \\
\sum M_{Z D}\left(V_{S}, Z_{S K}, \phi, \theta, \varphi, \delta_{S}\right)=0
\end{array}\right.
$$

VPP equilibrium calculations give the designer essential information about how the boat will sail in a specific situation. The main disadvantage is related to the information that is missing outside this specific sailing point. For instance, even if the VPP generated information could be considered as physically true, different questions arise. Is it possible to reach this specific state? Will the boat be able to accelerate to reach such equilibrium sailing point? How can the boat be sailed to reach such state? Is it easy or difficult to sail in such situations?

All the questions proposed above could be assessed using time-domain calculations, solving Newton's laws of movement in a time-dependent scheme. As explained in the sixth chapter of [156], everything is determined by solving a system of secondorder non-linear differential equations (presented in expression 3.2) using numerical tools and schemes which will be discussed later.

$$
\left\{\begin{array}{l}
\sum F_{X D}\left(Z, \phi, \theta, \varphi, \dot{X}, \dot{Y}, \dot{Z}, \dot{\phi}, \dot{\theta}, \dot{\varphi}, V_{A S}\right)=\ddot{X} m \\
\sum M_{X D}\left(Z, \phi, \theta, \varphi, \dot{X}, \dot{Y}, \dot{Z}, \dot{\phi}, \dot{\theta}, \dot{\varphi}, V_{A S}\right)+\sum M_{X S}\left(\dot{X}, Z, \phi, \theta, V_{A S}\right)=\ddot{\phi} I_{\phi} \\
\sum F_{Y D}\left(Z, \phi, \theta, \varphi, \dot{X}, \dot{Y}, \dot{Z}, \dot{\phi}, \dot{\theta}, \dot{\varphi}, V_{A S}\right)=\ddot{X} m \\
\sum M_{Y D}\left(Z, \phi, \theta, \varphi, \dot{X}, \dot{Y}, \dot{Z}, \dot{\phi}, \dot{\theta}, \dot{\varphi}, V_{A S}\right)+\sum M_{Y S}\left(\dot{X}, Z, \phi, \theta, V_{A S}\right)=\ddot{\theta} I_{\theta} \\
\sum F_{Z D}\left(Z, \phi, \theta, \varphi, \dot{X}, \dot{Y}, \dot{Z}, \dot{\phi}, \dot{\theta}, \dot{\varphi}, V_{A S}\right)+\sum F_{Z S}\left(\dot{X}, Z, \phi, \theta, V_{A S}\right)=\ddot{X} m \\
\sum M_{Z D}\left(Z, \phi, \theta, \varphi, \dot{X}, \dot{Y}, \dot{Z}, \dot{\phi}, \dot{\theta}, \dot{\varphi}, V_{A S}\right)=\ddot{\varphi} I_{\varphi}
\end{array}\right.
$$


The nomenclature used in expression 3.2 is also the same as the one used in expression 3.1 also including:

- I for inertia along the different rotation axis.

- $V_{A S}$ for all the adjustment variables that could be changed through the time history.

- $m$ for the boat mass (instead of using $\Delta$ that could lead to confusion).

During time-domain calculations, settings and adjustments have to be set and changed along the duration of the simulation. A proper strategy regarding how to manage them has to be made before the calculations are done. In fact, sail trimming and handling autopilots or prescribed strategies have to be implemented in order to reproduce the reality on-board. Such time-domain calculations are used to obtain information under specific circumstances that help the decision-making process. Using autopilots is a clever way to manage the settings control over time but is not always enough. For instance, even if the autopilot is sufficiently intelligent it will never represent the sailor with complete fidelity.

The next iteration of the time-domain dynamic VPP seems clear, if the interaction with the actual sailor is possible, then with one piece of software all the different situations could be examined. This allows obtaining a general purpose dynamic tool that will be handled by the actual user of the designed boat or a combination of sailors and autopilots, which will be explained later in this chapter. To develop the actual general-purpose tool (the simulator), some changes over the actual time-domain VPP have to be made. Instead of using a prescribed maximum duration of the time history (some seconds, some minutes), the new tool has to be able to handle long enough calculations to reproduce, for example, a whole regatta. The calculations have to be done as fast as possible, at least faster than the duration of the time step of each iteration, and reproducing the boat behaviour as closely as possible to the real boat. The tool has to be ready to interface with different elements to improve the relation between the tool and the user, such as VR engines, user interfaces, a hexapod to reproduce boat accelerations, etc. The integration of all, or some, of the elements cited before is what is called "the physical simulator".

The fidelity of such a tool relies heavily on the calculation process and the models used in the dynamic VPP. A fast and accurate time-domain calculation is needed to 
ensure this simulator is useful for the designer as well as the sailor. To achieve this main goal two factors have to be retained:

- To choose a fast and stable numerical scheme to solve expression 3.2.

- To develop proper forces and moments models that describe, quickly and accurately, the physical reality of the boat.

Another interesting use of the time-domain calculations, outside the VPP envelope but related to it, is the use of unsteady Computer Fluid Dynamics (CFD) methods to perform specific analyses. For example, in the paper by L. Mazas et al. [107], they present a RANS hydrodynamic analysis of two different foil configurations of an IMOCA 60 boat in a calm water simulation (like an equilibrium calculation in a VPP analogy) and in 5th order Stokes regular waves (purely time-domain situation). Offshore boats such as the IMOCA 60 will normally sail in waves rather than in calm water. This is the reason why the information extracted from the time-domain CFD simulation in waves combined with stationary information gives the designer a better general picture of the real performance difference between configurations. This information then can be used later to generate suitable VPP forces models.

\subsection{Physical Models for Forces and Moments}

As previously observed, two key elements are the most important foundations of the core of the simulator. The elements in charge of reproducing the physics behind the boat are the forces and moments models. Such models could be classified into three main groups:

- Interpolated models.

- Analytical models.

- Other models.

Ruiz de Elvira in his PhD thesis [156] proposes another classification: the models generated using experimental tools and the ones generated using numerical tools. From the data source point of view, this is the best way to classify them, but from the VPP code implementation point of view, the classification presented before is more convenient. Some of the methods and techniques explained below will be implemented within PASim for the Moth foiler boat and used for different simulations (please refer to Appendix B, C and D). 


\subsubsection{Interpolated Models}

The first group of forces models mainly used in a VPP are the ones that rely on a tabulated set of data. This set of data has to be as complete and accurate as possible. Because data collection is time-consuming and expensive, the testing or numerical simulation matrices have to be chosen carefully. If the data available is sparse, data management techniques could be used to expand or generate sets of data. The tabular data could be obtained using experimental techniques (mainly towing tank and wind tunnel testing) or numerical tools (CFD software ranging from potential codes to Reynolds Averaged Navier Stokes codes (RANS), etc). Normally, the data collected with the numerical tools mentioned here needs special pre and post-processing and a huge amount of computational time. After the data matrix is generated, an interpolation scheme has to be selected. The interpolation methodology should be chosen according to the shape of the data. The main focus when using interpolation schemes in the simulator is calculation speed without losing accuracy. The interpolation methodology used has to offer enough accuracy, to keep the simulator capabilities for reproducing the reality, with the fastest execution per time step. It could be easier to consider only one interpolation scheme that can fit different data topologies, like cubic splines, but this approach could be computationally expensive. A boat with a complex set of appendages and different sailing modes including a step change (for example transitioning from non-foiling to foiling) could lead to a massive amount of interpolation operations. It is then paramount to choose the method accordingly. For example, a set of data which shows a clear linear trend could be modelled using a least-squares fitted line. A general and robust interpolation strategy for sets of data for VPP is proposed by Ruiz de Elvira in the Section 5.2.4 of his PhD Thesis [156] and is the one that will be used across this work.

Another important aspect is how the data is handled and prepared for interpolation. In order to simplify the task of finding the equilibrium, data smoothness has to be kept for VPP equilibrium calculations. Sometimes, even if the data presents a trend or a topology that could suit a simpler interpolation scheme, it is preferable to choose a more complex one that will ensure smoothness in the derivatives.

When the boat presents two modes of sailing (with a foiling boat, for example, the boat could sail in displacement mode or in foiling mode) some strategies have to be set. The data near the transition point of sailing modes will not be smooth enough to easily solve the equilibrium of forces and moments near such a point. In this case two sets of data, one for each mode, could be used and the solver should be forced to 
calculate in both modes to find a solution; first in the potentially slower boatspeed and then when the transition is reached (hull flying or foiling condition), an autopilot should force the solver to calculate in the faster one. The whole strategy is described in the 8.2.3 section of [156].

Regarding data smoothness for time-domain calculations, even if it's not as important as for the VPP equilibrium calculations, some degree of it could be very useful. When the data is complex and a complicated interpolation scheme is needed, for the sake of computational speed, new strategies should be used. As explained in [156], J. Ozanne used a method for subsequent interpolation in situations when the spline coefficients cannot be calculated in advance, as it is the case of a multidimensional forces and moments model with multiple dependent variables. The method consists of generating a dense, smooth, regular grid based on the initial data using the most appropriate interpolation scheme before the time-domain computation. At runtime, a low degree polynomial interpolation is used between the points in the grid. This method provides fast interpolation and low errors when the grid is dense enough.

Another important aspect when dealing with tabulated data is the need for fairing for the sake of smoothness. Surprisingly, the human brain is capable of detecting trends and patterns when looking at data representations. After collecting all the data from experimental or numerical sources it is important to identify single points that are clearly outside the general trend of the data series. Such discrepancies may come from errors in measurement or numerical errors. If the discrepancy is obvious and the set of data is big enough, then it is advisable to eliminate such a point from the tabulated data. But, if every measure taken is double checked, a deep understanding of the physics of the problem will also help to discern if the discrepancy is related to measurement or numerical errors or if it is a part of the actual physical phenomenon. For example, the lift and drag anomaly generated when a sailing-boat keel is close to the free surface for a certain Froude number range (boat speed range)

\subsubsection{Experimental Data}

When studying sailing boats, there are two main sets of forces that describe the problem. From the fluid perspective, there are two of them travelling across the boat. In fact, the movement of the sailing boat is the consequence of the interaction between the forces and moments generated when the submerged part is travelling across the water and the forces and moments due to the emerged part of the boat travelling across the air. An experimental procedure has to be designed to measure both sets of forces. 


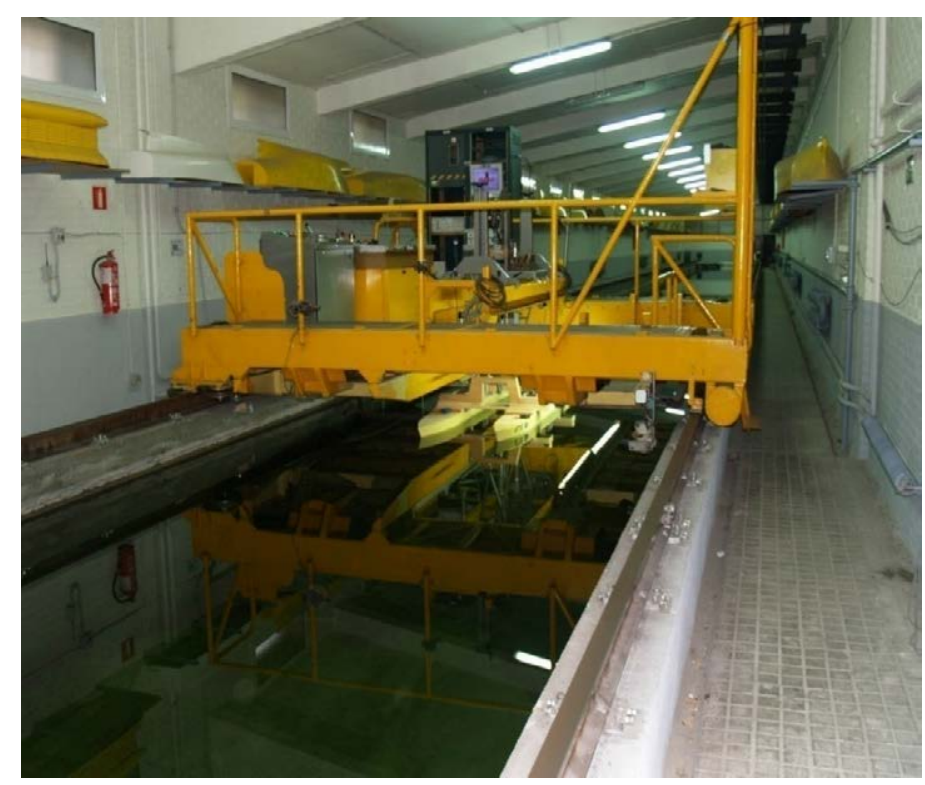

Figure 3.1: CEHINAV Towing Tank Facility in the ETSIN-UPM in Madrid. Photograph by CEHINAV Research Group

In the case of the hydrodynamic part, an extensive work on how to measure hull and appendage forces under the influence of an incoming flow has been developed since the first studies of boat towing tank testing developed by William Froude in the 19th century [61]. An example of a towing tank facility is presented in figure 3.1.

Regarding the aerodynamic testing, as explained by Baals and Corliss in their book "Wind Tunnels of NASA" [6], one of the first machines built to measure aerodynamic properties of a body travelling across an air stream was the Whirling Arm developed by Benjamin Robins in the 18th century. In 1871, Frank H. Wenham was credited as the first person to design and operate a wind tunnel. Proper wind tunnel testing of sails appeared later at the end of the twentieth century.

Towing Tank Testing: The towing tank specific testing procedure for sailing boats was developed in the early 1930s by Professor Davidson and was presented in his 1936 paper [42]. New techniques have arisen since Prof. Davidson's work, but they share the same physical principles. A good analysis of such techniques can be found in Parsons's paper from 1998 [129].

Regarding the simulator, the data acquisition process remains the same as for VPP equilibrium calculations. A very interesting and deep analysis of towing tank data acquisition for VPP calculations is presented in section 4.3 of Ruiz de Elvira's thesis [156]. 
The main concerns presented by Ruiz de Elvira are still valid for the simulator purpose, mainly the ones related to repeatability and accuracy. The model has to be well built and well finished respecting the dimensional tolerances, with the proper scale adapted to the specific towing tank where the test will be carried out (the biggest model accepted but avoiding wall interferences during each test). The model building techniques have to minimize model deformations. All the measurement equipment has to be calibrated and the expected forces acting on the boat must be within the dynamometer specifications. Finally, the testing technique has to match the needs of the designed testing matrix (semi-captive or fully-captive model technique, etc).

The test matrix is the main element that affects the interpolation speed. A balance has to be found between accuracy and the number of variables that compose the testing parametric space. For example, in a foiling boat, enough data must be obtained for the displacement sailing mode while maintaining simplicity. Sometimes variables could be ignored and the number of levels of interpolation reduced, gaining more computational speed for a minimal accuracy loss.

Wind Tunnel Testing: Regarding the aerodynamic data, the main facility used to collect it is the wind tunnel. A deep analysis of the typology of the different wind tunnels is presented in chapter 4 of Patricia Izaguirre $\mathrm{PhD}$ thesis [83]. It explains how the Atmospheric Boundary Layer tunnels or Environmental Tunnels are used to test sails.

These tunnels are capable of reproducing the earth's natural boundary layer and the wind profile in which the sails will travel. Because the boat is moving, the sail will travel under the influence of a combination of two different winds, the true atmospheric one and the one generated by the boat speed. Such a combination is called Apparent Wind (described by Tanner in [169]) and presents a specific aspect that has a huge impact on the sail's behaviour and performance, the wind twist. It is the consequence of having one of the two wind vectors constant with height (wind generated by the boat speed) and the other variable (wind gradient due to the presence of the atmospheric boundary layer), forming different resultant wind vector with a different angle of incidence for each height along the span of the sail, as explained in section 3 of Richard Flay's paper [55].

In order to solve such a problem, special Atmospheric Boundary Layer tunnels were developed. The concept of a Twisted Flow Wind Tunnel (TFWT), in which both velocity profile and twist profile could be reproduced, was originally developed 


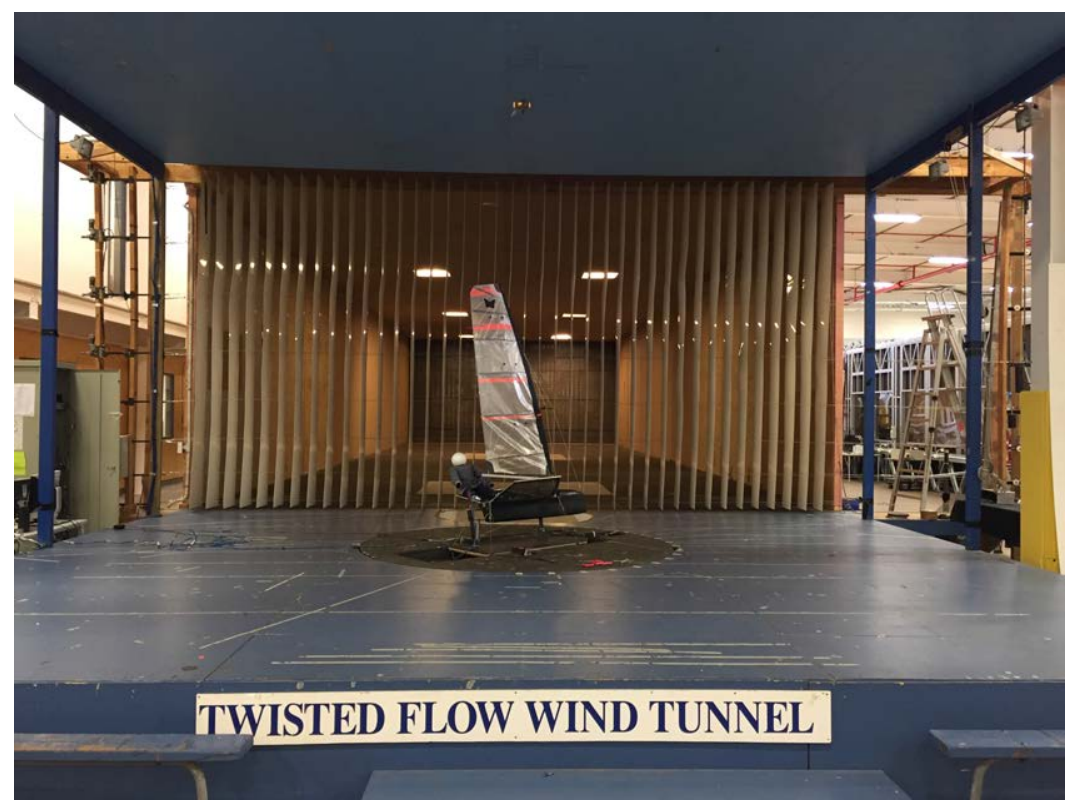

Figure 3.2: Twisted Flow Wind Tunnel of the University of Auckland

by Flay et al. for the New Zealand America's Cup Challenge in 1995 [56]. The actual TFWT of the University of Auckland is presented in figure 3.2.

In this case, an experimental campaign is very similar in comparison to a towing tank one, except with its own associated problems. For instance, the model geometry has to be a scale version of the real one. Not only the geometric aspect of the sails and rig has to be reproduced but also the stiffness of the sail, mast and rig must be scaled down. Mast and rig and sail deformations are a consequence of the coupling between the aerodynamic loads, the structural response and input tensions (trimming). Such deformations strongly affect the sail shape, this is the reason why when the stiffness is not properly scaled wrong aerodynamic forces and moments are obtained.

From the VPP equilibrium calculation point of view, the only information needed is the optimal trim set-up for each apparent wind angle (sometimes a de-powered trim set-up is also needed but analytical de-powering schemes could be used instead).

One of the main aims of the simulator is the capacity for user interaction. For the hydrodynamic elements (such as a lifting surface like a rudder or a foil), the layers of interaction and adjustment range from one variable up to a maximum of three (angle of attack, cant and rake) and they are normally related to the angular position of the element. For a rigid wing sail, such layering is very similar, mainly related to wing elements positions (twist, camber...). However, for a cloth sail, the large number of user inputs available, intrinsic features, such as sail and mast stiffness, and apparent 
wind speed combine to a large number of forces. To allow full control of a cloth sail in the simulator, the number of tests to be carried out is huge. For a foiling boat, not only the boat attitude is important (Pitch, heel, yaw, ride height, etc.), but also all the variables related to sail trim and control (mainsheet, traveller, cunningham, etc). This leads to a massive number of variables and interpolation layers with calculation times slower than the iteration time step. If the calculation time is bigger than the time step, the simulator has a time lag between proper time execution and actual calculation making the simulator useless for real-time usage, but not useless for design simulations (it can still be used to explore crew-work manoeuvres using script files and autopilots, for example) . The solutions to this problem could be:

- Choosing an efficient interpolation methodology like the one used by J. Ozzane.

- If some trimming options are not really used while sailing, these variables could be fixed.

- If other aspects of sailing will be explored, just very basic trimming schemes could be used (mainsheet only or de-powering scheme that reduce the trimming effort to one or two variables).

- The use of analytical de-powering schemes like the one presented inside the ORC IMS VPP (Flat with a twist function and reef parameters) [33] combined with an optimum trimming model based only in APW and boat attitude. A trimming and de-powering autopilot controlling such analytical schemes could be used if needed.

These solutions always present a trade-off between accuracy or level of realism as opposed to fast computation time, but they are only needed in real-time simulation use.

\subsubsection{Numerical Data}

Another source of information to generate forces and moments models are fluid dynamics numerical methods. As with the experimental data, the methods considered here are the ones that could be found as stand-alone codes outside the VPP. Such codes normally need specific pre-process of the geometry analysed and results postprocessing, resulting in the same type of tabular data as generated using experimental methods. In this case, accuracy is paramount and all the information has to be kept. 
The only real limitations regarding the amount of data to be collected are the computational resources available compared to the needs of each specific method, and the levels of complexity of the physical model with the number of levels of successive interpolation needed. Other simpler or more efficient methods (Lifting Line, Vortex Lattice, etc) that can be implemented directly into the VPP for direct execution in time-domain calculations will be analysed later.

Inside this category, different methods can be found, from faster but less accurate potential ones to slower but more accurate viscous methods. They are listed below.

Panel Methods: A wide variety of panel codes are used to generate forces and moments information. These tools are based on the assumption that the velocity of an irrotational and inviscid flow derives from a scalar potential, allowing the continuity equation to be reduced to the Laplace equation. Using the superposition principle for linear equations, the flow problem can be written as the sum of an external uniform flow and a body perturbation flow. The Laplace equation can be solved using a distribution of elementary solutions (associated to each panel) on the problem boundaries that defines the body analysed and satisfying all the boundaries conditions associated with it, as explained in Chapter 3 of Katz et al. "Low-Speed Aerodynamics" book [86]. These elementary solutions can be a source, a doublet (combination of source and sink) or a vortex.

Panel methods can be used to solve two-dimensional problems (such as obtaining the pressure distribution of an airfoil section) or to solve three-dimensional problems (such as obtaining the pressure drag of a hull including free surface specific panelization like the method presented in Raven's thesis [148]).

The main disadvantage is that these panel methods only solve non-viscous flows. All the viscous effects have to be taken into account using different solutions, such as:

- For hull resistance analysis, a dynamic wetted surface determination that allows viscous correction by using a viscous line and a form factor.

- For lifting surfaces, stripping the surface in those cases where there is a significant variation in the chord length along the span, a similar procedure can be used with calculated viscous drag coefficients and an estimated form factor.

- For lifting surfaces or airfoil section calculations, a more detailed approach can be used such as the one implemented in Mark Drela's XFOIL program [47], offering a strong coupling between the boundary layer equations and the 
inviscid panel method. When strongly coupled, a single solution of the boundary layer equations gives the complete solution including the viscous effects on the pressure distribution.

These methods, even if they present less accuracy than others (for example RANSE), work well with attached flows in lifting surfaces or slender hulls. With technological advancement, the advantage is that currently they are very lightweight computationally and a large amount of data can be generated very fast. This means that such methods can be used when the level of accuracy required is lower, such as for preliminary design tests or multiple geometry comparisons, taking into account their limitations.

Euler Method: These methods offer a different approach to solving a non-viscous flow. They solve a simplified version of the Navier-Stokes (NS) equations. This simplification is called the Euler equations, which are the NS equations without the viscous and the heat conduction terms. Unlike the panel methods, they admit vorticity and can capture flow discontinuities (shocks) as explained by Baker et al. in [8]. Because the fluid region around the studied bodies is discretized (as opposed to the panel method where the body itself is discretized) wake interactions between different bodies can be captured. Used in aerodynamics for supersonic flows, they require more computer resources than other potential methods. They don't offer an increased level of accuracy in comparison to panel methods (except for wake interaction) for the problems usually associated with the physics of a sailing boat. This is the reason why they are not widely used for VPP data generation.

RANSE Method: When high accuracy is needed and enough computational resources are available, the most commonly used method is the Reynolds Average Navier Stokes Equations (RANSE). With a similar approach to the Euler method, RANSE methods can solve Navier Stokes equations using some simplifications, such as a Reynolds-averaged form of the NS equations combined with a model of turbulence (solving even some of the turbulences scales is too expensive computationally from a practical point of view). Unlike the Euler method, it is capable of solving full viscous flow problems directly. It can solve (virtually) all the flow problems around elements found in a sailing yacht, ranging from a specific geometry like a foil fully submerged, up to the whole boat geometry with the rig and sail in the presence of a free surface (using multiphase techniques like the Volume of Fluid (VOF)). It can be used to simulate steady or unsteady problems. In fact, RANSE methods are not 
only used for data generation to build forces and moments models but also to study the time-domain behaviour of some configurations in certain situations (with a higher computational cost than the steady simulations). The main problem is that RANSE results are dependent on the quality of the domain discretization (mesh quality and size). When time-domain simulations are carried out and imply a great amount of movement, special meshing techniques have to be used (such as mesh morphing or Overset mesh) resulting in higher computational cost. From the accuracy point of view, direct RANSE time-domain unsteady simulations used during time-domain VPP execution time would seem the best solution as the main source of forces and moments for the simulator. But, for the moment, it is not possible to match simulation time to execution time because of the computational and time costs. For data generation (associated with the same problems explained before, related to interpolation complexity in the model) it is one of the best numerical tools available. An example of RANSE method applied to foiling catamarans can be found in Caponnetto et al. [25].

An in-depth analysis of such methods relating to their use for VPP forces model generation is presented in section 4.5 of [156]. A comparison between different methods for force and moment generation for a VPP of a foil (kite foil) is presented in Ocaña et al. [124] and could serves to illustrate the previous sections.

\subsubsection{Special Considerations}

It is important to remark on two considerations regarding interpolated forces and moments models. The first is to carefully select the frame of reference for forces and moments before deciding the interpolation scheme. This is important in order to avoid more changes in frame of reference while calculating and adding complexity to the interpolation strategy. In fact, the VPP used and developed for the simulator, PASim, which is based on the work of Ruiz de Elvira and described in section 2.2 of [156], could work in four main systems of reference and can be adapted for taking into account even more. These four frames of reference refer to the different elements involved:

- Local Axes for each calculated yacht element (keel, rudder, hull, sail plan, foils, etc), oriented with the element geometry. It is specially adapted for physical model generation and is the simplest to use while acquiring the data of individual elements. 
- The Boat Axes: fixed to the boat and defined in the same way as used in naval architecture. Mainly used for VPP equilibrium calculations (orientation is based on the boat geometry).

- Track Axes, oriented with the movement of the boat but with the origin in the boat and associated with the boat hydrodynamics (Normally and historically used in VPP calculations). The frame of reference that is preferable to use while acquiring data of a group of elements together for the sake of simplicity.

- Wind Axes, oriented with the apparent wind direction (at the selected reference height of the aerodynamic model) but with the origin in the boat and associated with the boat aerodynamics.

Sometimes it is difficult, when using experimental facilities, to adapt the frame of reference of the measurement instrument to the one used in the VPP. For example, the data collected in the TFWT will use the frame of reference of their own 6-DOF scale.

Another important consideration is that the information collected using these methods is usually steady state. As previously explained in the RANSE method paragraph, generating unsteady time-domain data with high accuracy in execution time is currently impossible. Some corrections of the models will be necessary. For example, the use of analytical unsteady correction functions for the steady state data like indicial functions for lifting surfaces as will be explained later.

\subsubsection{Analytical Models}

When the physics involved can be described using less sophisticated models but maintaining some level of accuracy, they can be solved using analytical models or more complex but computationally lightweight numerical methods such as generalized Lifting Line or Vortex Lattice Methods. To describe some of the phenomena involved in sailing, it is possible to use simplified physics, losing accuracy, but without losing the sense of realism when the simulator is used. These types of models allow swift testing, without any major schedule, new geometries and configurations or even more specific situations regarding handling or communications on board.

They allow the retention of computational resources during time-domain calculations that can be allocated to other tasks, more computationally demanding, executed in the same time step. 


\subsubsection{Analytical Models}

Since the first use of VPPs, analytical models have been implemented as a very practical option when more complex numerical methods were not available. For instance, the first VPP developed by Justin E. Kerwin in his 1976 paper [87] was based on simple analytical models combined with simple interpolation schemes (to obtain some coefficients needed to calculate such models) accounting for:

- Hull Upright Resistance.

- Hull Upright Viscous Resistance.

- Resistance increase due to Heel and Side Force.

- Hull Stability.

- Sail Forces

Kerwin showed that with a low level of complexity it was possible to obtain reasonable results. Following the same philosophy, it is possible to obtain reasonable results in the simulator using analytical models (as enumerated hereunder), but knowing the assumptions made in each one of them.

Analytic Hull Models: Fundamentally associated with the Froude decomposition of the resistance, (equation 3.3), the analytic forces models for the hull give an estimate of the residuary drag $\left(C_{R}\right.$ or $\left.R_{R}\right)$.

$$
C_{T}=C_{F f p}+C_{R}
$$

Where $C_{T}$ is the total drag coefficient, $C_{F f p}$ is the flat plate friction coefficient and $C_{R}$ is the residuary drag coefficient.

Such models are calculated using geometric data from the hull and could also be dependent on the speed of the boat. Historically, the most commonly used are:

- The model developed by Kerwin [87].

- The model developed by Hazen [76].

- The Delft Systematic Yacht Hull Series (DSYHS) developed by Keuning et al. (a regression based on an extensive towing tank campaign and with the last version presented in 2008 in [89]) 
- The model implemented by ORC in their VPP [33].

To give a more accurate estimate, all these models are normally completed with drag corrections either due to the heel or trim of the boat or due to added resistance in waves. Some models also offer an estimate of the hull wetted surface (like the DYSHS) if such information is not available.

Analytic Lifting Surface Models: The lifting surface simple model could be implemented for a first estimate of the forces and moments generated by the appendages (Rudder, keel, generic hydrofoil, etc). Some of the models discussed before (DYSHS) also include an estimate of the drag of the appendages. However, for a proper calculation of lift, the analytic solution for an elliptical distribution of the Lifting Line combined with a strip drag calculation based on 2D section information (calculated with an external 2D panel method) can offer good results whilst being very inexpensive computationally.

Analytic Sails Models: The International Measurement System (IMS) model implemented in the ORC VPP [33] is the most complete analytic sails model available based on basic geometric parameters of the sail plan. It is based on an extensive wind tunnel testing campaign with different combinations and configurations of the sail plan.

In fact, the model is able to combine each specific geometry and coefficient of each individual sail, in the defined sail plan, and calculate a general coefficient taking into account the sails in use working together. It also takes into account the blanketing effects between them, etc.

After obtaining the global coefficients, the global forces are calculated and the centre of effort is estimated. It also calculates a coefficient accounting for the efficiency of the sail plan. To take into consideration the de-powering normally used while sailing, the IMS incorporate two elements: flat (with a twist function depending on flat value) and reef, that are intended to work together similar to the way de-powering works on-board.

\subsubsection{Numerical Methods Solved in Execution Time}

It is possible to run, in execution time, simple fluid numerical methods. For example, it is possible to run more complex lifting line than the analytical solution of the elliptic distribution or even more complex numerical schemes such as the Vortex 
Lattice Method (VLM). Both techniques are widely used to calculate hydrodynamic lifting surfaces and sails (One of the most used codes for aerodynamics calculations, North Sails "Flow" [157] uses VLM coupled with a structural code called "Membrain" to calculate flying shape sails coefficients).

Complex Lifting Line: The lifting line theory was first presented by Lanchester in 1907 [94] and then Prandtl in 1918 [142]. It is a mathematical model that aims to predict the span load over a three-dimensional lifting surface. The initial theory used a single bound vortex usually positioned on the quarter chord line of the planform associated with a vortex-wake where Kutta-Joukowski and Helmholtz's second theorem are applied. Modern implementations take into account different geometrical aspects such as chord and twist spanwise distribution [138] up to a generalized version like the one presented by Guermond et al. in [68] or the one presented by Phillips et al. in [139]

The main disadvantage of such theory is that it is limited to high aspect ratio lifting surfaces with small loading conditions as explained by Van Dyke in his 1964 paper [179].

One of the main advantages of the chord distribution implementation is the way it easily adapts to viscous drag strip methods and unsteady indicial correction explained in section 3.3.2.4. Other developments of unsteady calculations are available such as the scheme presented by Guermond et al. in [69].

VLM: For more complex lifting surface geometries such as complex foils ( $\mathrm{J}$ foils, IMOCA 60 Dali foils, etc.) the most convenient method that is easy to use and can run in execution time is the Vortex Lattice Method first presented by Falkner in [50].

Instead of using one single bound vortex or a combination of it along the span, as in the lifting line method, such vortices could also be distributed along the chord. Associated with each of the bound vortices, a system of trailing edge vortices is also considered for each element.

Following this idea, the lifting surface could be defined using a succession of horseshoe vortices associated with control points forming panels. The geometry is described by such panels forming a lattice of vortices. Then it is expressed numerically, after applying the flow-tangency condition and Biot-Savart Law, using an algebraic system where the vortex strengths are the unknowns. The solution reached with this algebraic system gives the lift force and the induced drag forces. For a complete 
forces calculation, viscous forces could be added by using strip theory with data from Drela's XFOIL [47], for example.

One very interesting implementation that allows the pre-computation of all the problem matrices before execution time is the compact VLM implementation by Bunge et al. [22]. For fully submerged appendages this is the ideal method regarding accuracy versus execution speed because it allows the use of lifting surfaces with control elements (flaps, rotating surfaces, etc) reducing the calculation to matrix multiplication in each time step, resulting in small calculation times. It is coupled with a strip theory code and it could be modified to take into account end-plating effects from the hull, for example. One of the main disadvantages is that when the lifting surface is not fully submerged and moving close to the free surface the submerged geometry varies with time and a new panelization is needed for each time step. This new panelization leads to an increase in computational time that may make the use of such a method inappropriate for execution time. A possible solution to this problem will be described qualitatively in 3.3.2.3. The other main problem is the free surface proximity effect in the lift distribution. Other schemes or corrections could be adopted to solve such problems, such as the ones based on the use of boundary conditions for horizontal hydrofoils working close to the free surface as explained by Thiart in [174] and [175].

\subsubsection{Numerical Methods Combined with Interpolation Schemes}

As introduced in the last section, when a piercing foil moves along the free surface and is solved with the VLM method, for each time step the submerged geometry changes and has to be re-panelled. For a geometry with a large number of panels, that implies the computation of large algebraic systems for each time step. From the computational point of view, the VLM method, even with such problems, is still a fast method and can be used for stationary calculations. But, for time-domain calculations, the computation of such large algebraic systems normally needs more time to execute than a reasonable time step (usually not much higher than 0.1 seconds). The VLM method can still be used if such matrices are pre-computed prior to the time-domain VPP execution time for a set of different foiling heights (from fully submerged to foiling mode). The problem is then reduced to some matrix multiplications (to obtain forces for three different heights) and a very simple interpolation scheme (six interpolations of three points each) of all the forces and moments of the foil. Such methodology could be extended to other models that allow pre-computation of some elements before execution time. 


\subsubsection{Unsteady Model Correction}

As noted previously, the VPP models usually rely on steady state data to generate the forces and moments needed to calculate both equilibrium and time-domain solutions. For time domain, the assumption of using steady information instead of unsteady could be considered inadequate. The boat dynamics and the force generation in the appendages over the time-domain simulation present a different behaviour than the one obtained using the steady force models.

In the first commercial sailboat simulator, the Virtual Sailing VS-1 presented in [12], a simple analytical correction of lift generation, proposed by Marchaj on page 207 of [102], was implemented. It was based on the assumption that the foil has to travel 6 chord lengths after a change in angle of attack to develop $90 \%$ of the full lift.

In the same direction as the one followed in the VS-1, in order to generate unsteady information for all the force models available for lifting surfaces in the VPP, the approach presented in [178] by Van der Wall et al. (with a generalized methodology presented in section 8.14 in [97] by Leishman) will be adopted. This approach consists of the calculation of an effective angle of attack based on the indicial response ${ }^{1}$ of 2-D sections using Wagner functions. The classic approach when using Wagner indicial functions ([182]) is to model the lift response for a step change in the angle of attack. But, it could be also used to model the lift response for a step change in the incidence velocity combined with a change in angle of attack.

Following Van der Wall et al. [178] the numerical scheme implemented takes into account two contributions (as explained in Theodorsens theory [173]). The first contribution is related to non-circulatory lift (added-mass term) due to the instantaneous acceleration of the fluid around the 2-D section. The second contribution is the circulatory lift which is time-dependent. It could be treated using a Wagner indicial function for a time-varying incident velocity and angle of attack in the time domain by the means of Duhamel superposition.

For the sake of simplicity in solving the Duhamel integral, the Wagner function is approximated using a linear combination of two exponential terms like the one Jones presented in [85] for the flat plate. A recurrent scheme is used to solve the Duhamel integral using the Simpson's rule as suggested in section 8.14.1 of [97]. This algorithm is efficient and fast, keeping all the system time history information only in the previous calculated iteration, with no need to retain any other information. A result of this implementation is presented in figure 3.3 showing the unsteady indicial

\footnotetext{
${ }^{1}$ The indicial function describes the system response when it is instantaneously submitted to a step function disturbance
} 
effective angle of attack calculation (in red) and the steady one (in blue) of a flat plate under the influence of a sinusoidal oscillation of the real angle of attack followed by suddenly fixing its value.

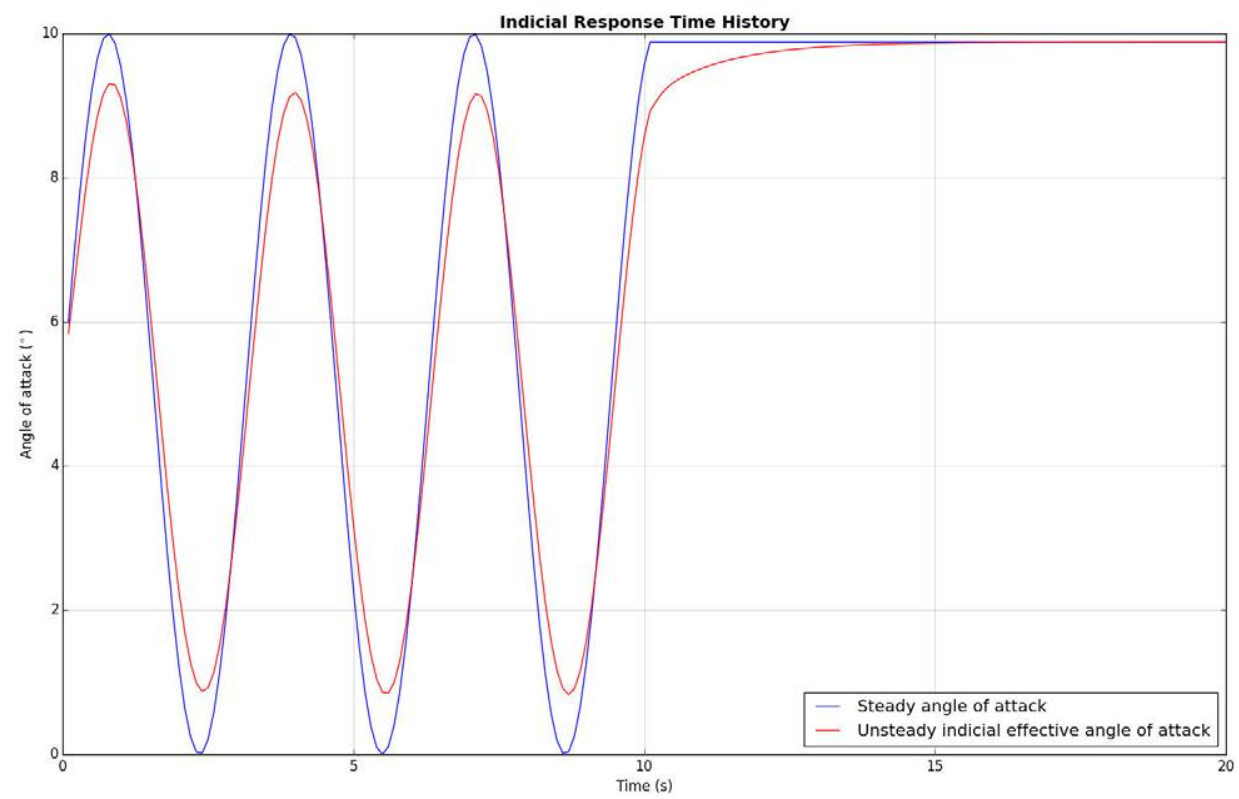

Figure 3.3: Flat plate effective AoA under the influence of a sinusoidal oscillation followed by suddenly fixing the value of the angle of attack

To adapt such a two-dimensional method to the three-dimensional geometries, three methods were developed and implemented within PASim:

- A strip methodology for use with the strip-based lift calculation models (Lifting Line, etc).

- A strip methodology with an effective Angle of Attack (AoA) chord averaged to obtain an approximation of a global effective AoA of the lifting surface.

- A fast method based on the calculation of the effective angle of attack using the mean chord.

Each approach allows the use of steady state generated data in an unsteady scheme maintaining computational efficiency. For the sake of accuracy, if numerical models are used in execution time and the calculation time is lower than the time step used in the time-domain calculations, the fully unsteady approach should be used. For 
example, the unsteady Lifting-Surface Method using Vortex Ring Elements as described in section 13.12 of the book by Katz et al. - "Low-Speed Aerodynamics" [86] (it can be used with fully submerged lifting surfaces with very little modification but the wake panelling implementation of this method cannot deal with piercing foils moving vertically in the free-surface). If the computational effort is too big to run a full unsteady model, the approach mentioned above can be used with one of the three options implemented using indicial functions.

The proposed methodology can be considered as a general approach to generate unsteady data for all the lifting surface models (from interpolated data to analytic and numerical schemes). More studies can be found for specific lifting elements available on board, such as the sails. Gerhardt et al., for example analyse the two-dimensional problem of the unsteady interaction between two sails in a yacht [64].

\subsubsection{Other Models}
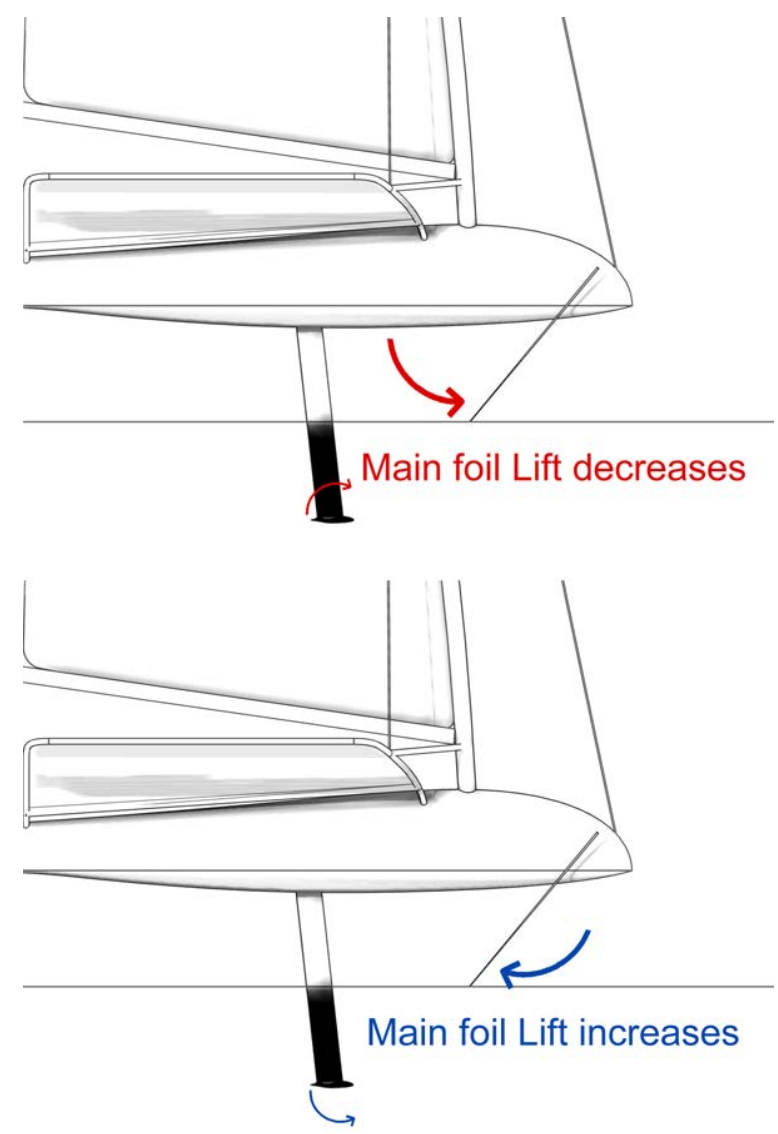

Figure 3.4: Moth Foiler Wand Control System Explanation 
Some special models have to be implemented to attend to specific needs of special boats or sailing modes. One of these cases would be when using lifting surface analytical models with a piercing foil. These simple models do not take into account the drag generated by the effect of the foil piercing the free surface (generating waves and spray) or by the horizontal part of the foil working close to the free surface (generating wave drag). These specific problems are included when using complex numerical tools such as RANSE codes combined with interpolation schemes. For simpler models, some analytical corrections can be used. In their Moth VPP [18], in Appendix C.3.4.3, Bögle et al. present some corrections based on appendage geometry or appendage coefficient correction based on existing data. More corrections on lifting surfaces can also be used, such as an effective angle of attack calculation based on the effect of the presence of a flap or a simple end-plating correction for piercing and non-piercing lifting surfaces.

Specific models regarding other elements have to be taken into account. For instance, the possibility of calculating a movable crew affecting the position of the centre of gravity of the boat and the righting moment should be implemented.

If the boat has some kind of foil control system or flap control system (such as the wand in the Moth foiler (Figure 3.4)), it has to be modelled. In Appendix A of [18], Bögle et al. present their analytical model based on forces and moments applied to the wand and the system geometric characteristics.

\subsection{Time-Domain Solver Numerical Schemes}

As previously discussed with the forces models, the other major element that has an important influence on the Simulator behaviour is the way the time-domain Second Order Non-linear Differential Equations system 3.2 is solved. PASim implements the following numerical schemes (from simple to more complex):

- Euler scheme.

- Backward Euler (Fixed Point) scheme.

- Backward Euler (Newton) scheme.

- Midpoint scheme.

- Runge-Kutta $4^{\text {th }}$ order scheme.

- Implicit Runge-Kutta $4^{\text {th }}$ order scheme. 
The details about each method and an explanation of how to implement the numerical schemes is found in "Numerical Recipes. The Art of Scientific Computing" [143], or other similar books about numerical computation.

The level of complexity (as ordered above) is directly related to the numerical order of the scheme and the level of accuracy and stability (Higher complexity equals higher order and higher level of accuracy and stability). The same order is similarly associated with code implementation complexity and computational requirements, higher order means higher implementation complexity and higher computational requirements.

The same problems explained for the forces and moments models are repeated here. The numerical scheme has to be chosen carefully, looking for the one that offers the best accuracy and stability versus its computational requirements, but always choosing a method that can be solved quicker than the actual time-domain iteration time step if the simulator needs to run in real-time.

A specific study has been carried out to illustrate such a dilemma. Even if the boat chosen as the example for the thesis is the Moth Foiler, the models used are the ones of a generic America's Cup 72 boat presented in chapter 8 of Ruiz de Elvira's $\mathrm{PhD}$ [156]. The reason for this was that when the study was carried out, the models of the AC72 were more robust and were already tested.

The study consisted of calculating, under specific conditions of True Wind Speed (TWS) and True Wind Angle (TWA) and reasonable initial values for the adjustment variables (but without using a pre-calculated VPP equilibrium solution for such values), a time-domain simulation using the different numerical schemes. The goal was to verify how the system evolved and whether it was able to reach an equilibrium state. The initial conditions were a TWS of 7 knots and TWA of 130 degrees. The initial boat speed was 11 knots and the boat was set sailing in displacement mode (not in foiling mode), behaving like a conventional catamaran.

For the first time-domain simulations, a large time step (0.5 seconds) was arbitrarily chosen to test the potential instabilities of the different numerical schemes in unfavourable conditions. The total amount of time simulated was set to 50 seconds. Then the calculated variables were plotted for the different numerical methods. Figure 3.5 shows the time history of boat speed in knots versus the time in seconds. The plots of the rest of the variables (heel angle, pitch angle, yaw angle, sinkage, AWS and AWA) are included in Appendix A. 


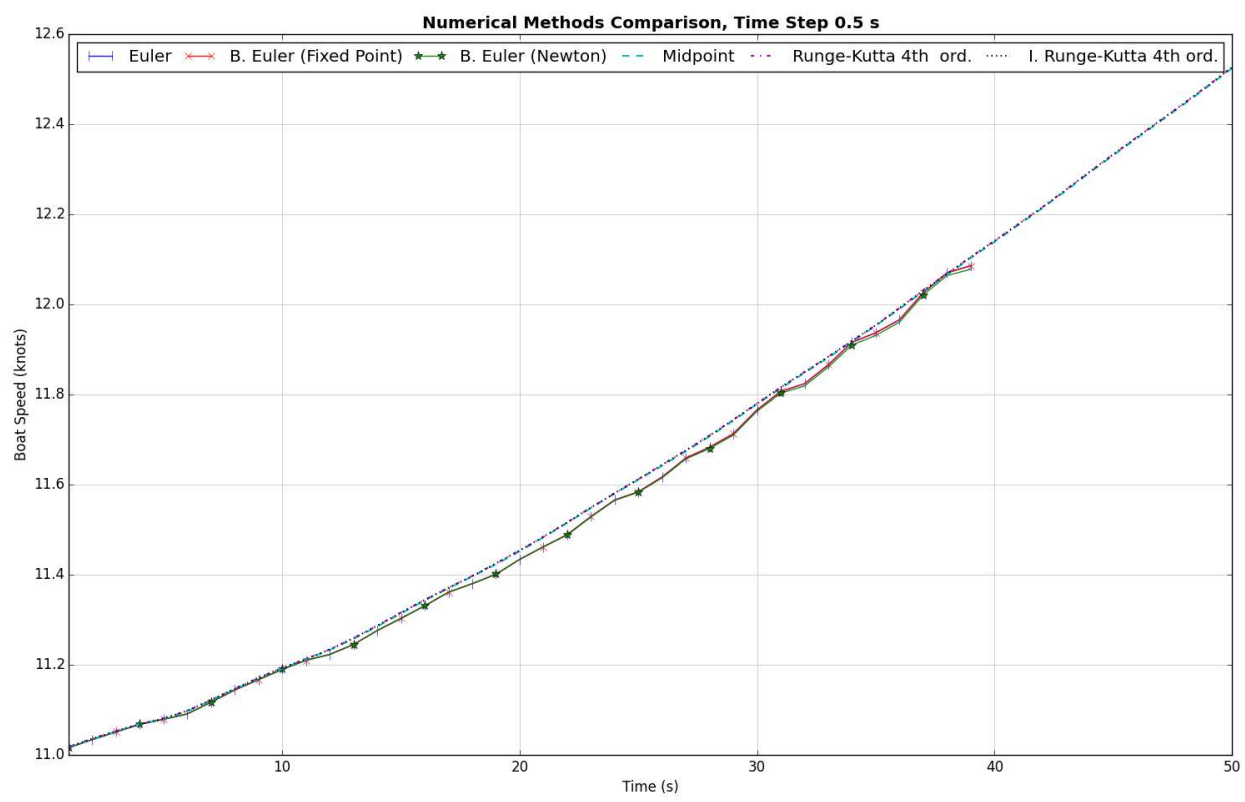

Figure 3.5: AC72 boat speed time history for the different numerical schemes

Because the initial values used for the AC72 time-domain calculations did not define a situation in which there was an equilibrium of forces and moments, in some of the calculated variables an initial physical oscillation was induced. In Figure 3.5 for BSP, low order schemes tend to start to oscillate (due to numerical error) to the point where the models fail to calculate after 39 seconds of simulated time. When higher order methods are used (Midpoint method and subsequent) the calculations didn't present oscillations in BSP and models were able to calculate all the simulated time. As shown in Appendix A, for the rest of variables, oscillations are present for all the methods, but for higher order the amplitude tends to decrease while for low order methods the oscillations amplitude increases especially for the sinkage. It is important to point out how the different DOF are coupled in the AC72. For example, when the boat is submitted to a variation in the pitch angle, the amount of vertical force generated by the foils is changed due to a change in the local AOA. This change in the vertical force modifies the sinkage of the boat affecting the lateral area, modifying the lateral forces and consequently changing the yaw and heel angles. If sinkage and pitch are plotted together, when using high order methods, the oscillations in both variables are out of phase, helping the boat's heave stability. If low order methods are used, due to the numerical induced oscillations, both variables start to enter in phase amplifying sinkage oscillations as it can be observed in Figure 3.6. 


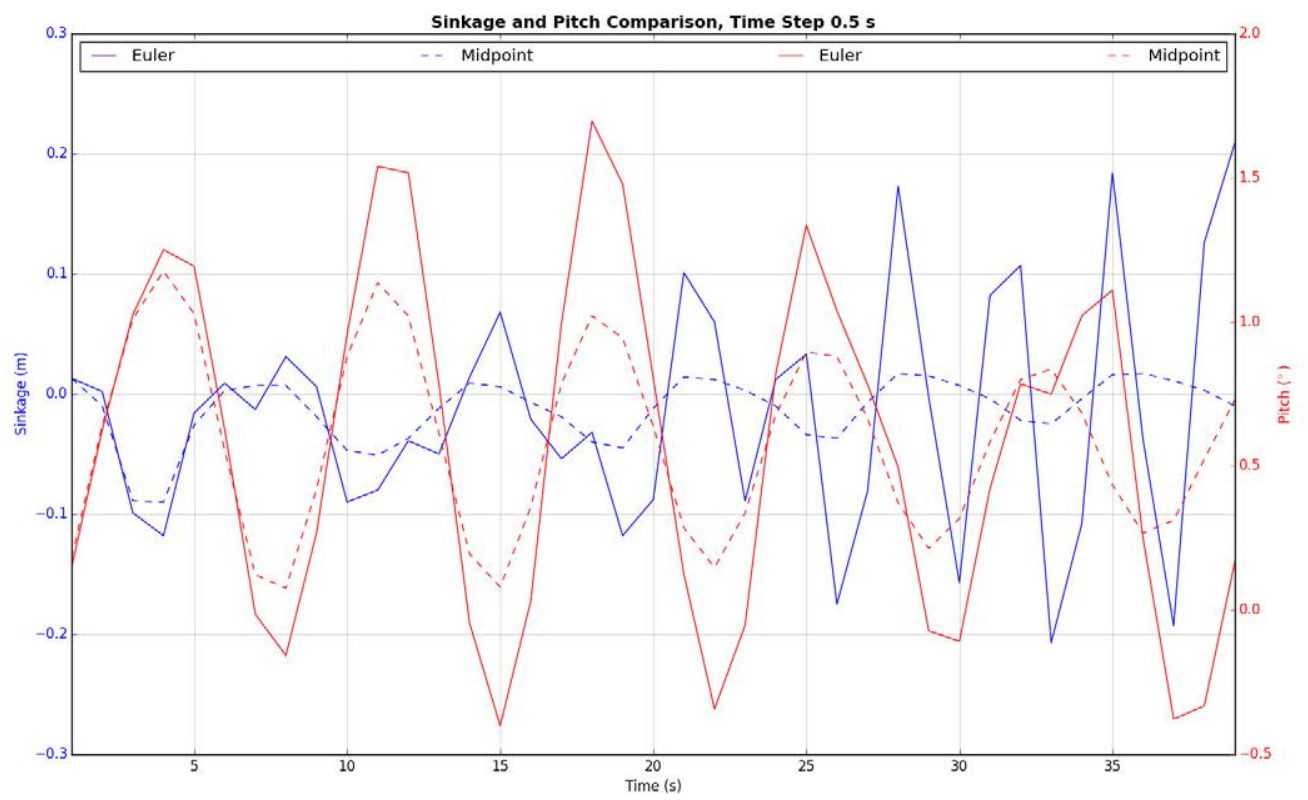

Figure 3.6: AC72 pitch and sinkage time history comparison using Euler and Midpoint methods

To verify if the aforementioned BSP oscillations and the amplitude increase for oscillations in the other variables, that appear when using low order schemes, are related to a numerical error, different time steps were tested for two different methods, the low order Euler method and the higher order midpoint method. As it can be observed in Figure 3.7 (the plots of the rest of the variables are included in Appendix A), when the time step for the Euler scheme is reduced to 0.1 seconds, the forces and moments models are able to calculate all the time history up to 50 seconds but the oscillations in BSP and the amplitude increase for oscillations in the other variables is still present. If the time step is reduced 50 times (from 0.5 seconds to 0.01 seconds) when using the Euler method, the oscillations in BSP disappear and the time history matches the one obtained using higher order numerical schemes. Proving that aforementioned oscillations are related to a wrong selection of the time step value for low order methods and purely numerical. The midpoint method was tested with a larger time step of 2 seconds matching the time history obtained with the same method and a time step of 0.5 seconds (Figure 3.7). This shows that higher order schemes could be used with larger time steps without losing accuracy for this particular case. 


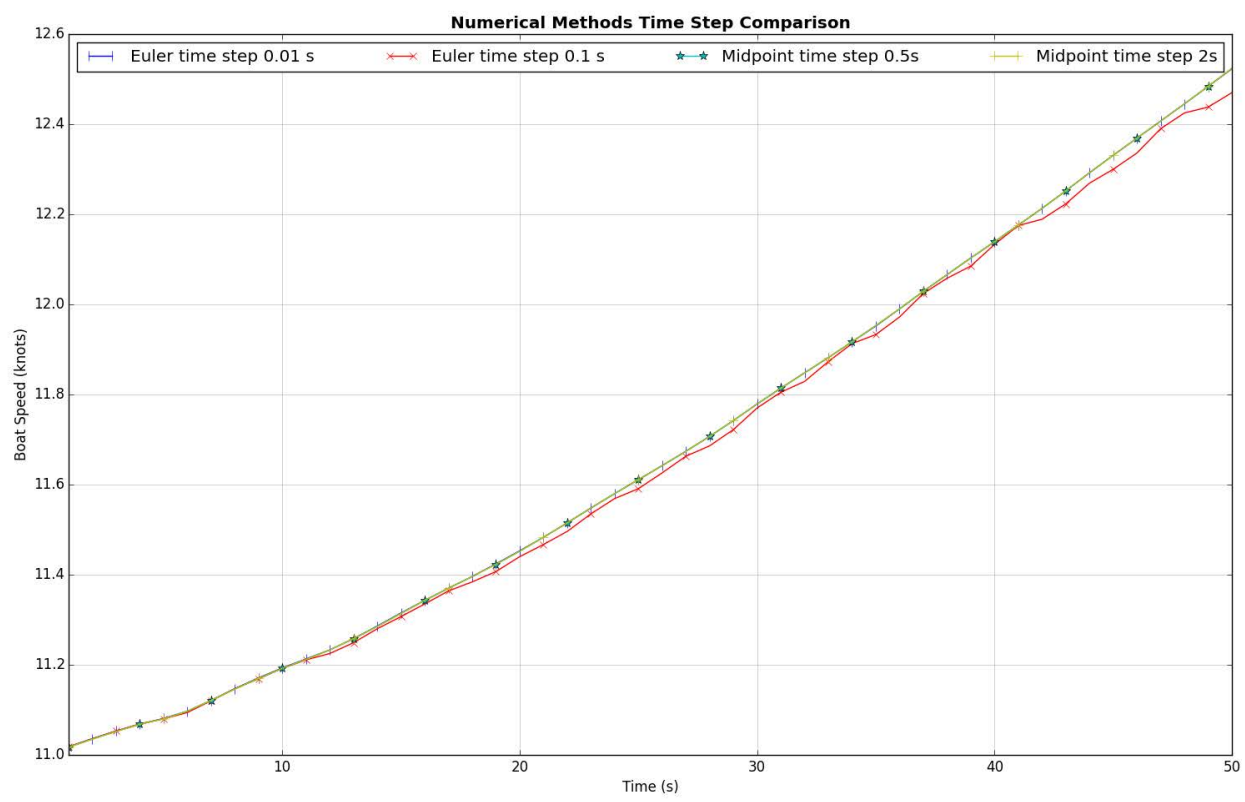

Figure 3.7: AC72 boat speed time history comparison using Euler and Midpoint methods with different time steps

The more reasonable selection for a numerical scheme seems to be the higher order method that implies the lowest computational effort. For the AC72, the midpoint method gives the same results as higher order methods such as 4th order RungeKutta. Such a method will be used as the baseline for different boats and situations.

Regarding the selection of the time step to use it is important to point out its dependency on the inertial properties of the boat, the computational time required by the calculation of all the forces and moments, the rate of motion cue generation needed for a proper acceleration reproduction with the Hexapod, the acquisition frequency of the interface that allows the user to control the simulator and the Virtual Reality (VR) engine calling rate for updating the boat state vector to the VPP .

A reasonable initial time step that normally satisfies all the exigencies and was tested with the example boat (Moth Foiler) chosen for the simulator is 0.1 seconds. Different computational time measurements when executing the Moth models are presented in Appendix C. They show that 3.3301326 seconds are needed for 1000 executions of a complete set of Moth force models (in this case, a selection of the simpler models described in Appendix D and characterising all the elements and parts of the boat) using a single thread execution scheme (please refer to task 7 in 3.6). Therefore, for executing one time the complete set of force models only 0.0033 
seconds are needed, showing that the execution time is below the time step chosen for the Moth and ensuring real-time execution. In fact, it gives extra time for executing the rest of the modules (motion cue generation, Virtual Reality (VR) engine, etc). Even if there is margin to decrease the time step (from 0.1 to 0.0033 seconds), when using higher order numerical scheme there is a point where the model did not offer better results (showed in Appendix A.2 figures). A sufficiently large time step that allows obtaining a proper solution with the selected numerical scheme is preferable in order to provide enough margin to fulfil the rest of the simulator computational needs when solving in real-time, minimizing the number of time steps that have to be calculated.

It is important to notice that for the selected VR engine (Unigine), described in Chapter 5, the refresh time for the boat state vector to be reproduced is fixed to a certain number of frames (images displayed by the engine) processed. The time step used has to be lower than the refresh time for a specific graphic-card/engine combination to minimize the error between what it is shown and what it is displayed, which is the case for the time step selected for the Moth. However, the VR engine can handle the odd skipped calculations that may happen sometimes in a non-real-time operating system, such as Windows, in an imperceptible way for the user.

\subsection{Autopilots}

One of the components that allows the time-domain VPP, and hence the simulator, to work without multiple human external operation (sailors using the simulator) is the autopilot. The idea behind the autopilot for a simulator is based on two different objectives:

- To handle the adjustments of the boat elements in a simulation without the need for user inputs. For example, in early design stage when analysing the dynamic behaviour of the boat under certain circumstances, the design team has to be able to mimic the handling and trimming of the boat using predefined data and specific algorithms to generate data fast and not depending on the design team sailing skills and judgement. When the simulator is used for training, the autopilots allow the simulator to be operated by a reduced sailing crew. For example, if the training is focused on improving steering of the helmsman, the other sailors in the boat can be replaced by autopilots. They have a large impact on the simulator usability and help to reduce the economic cost, becoming an essential part of the simulator. 
- To use intelligent autopilots combined with optimization algorithms to determine the optimum sequence of actions to obtain the best performance in specific regatta situations such as manoeuvres.

Autopilots are normally related to guidance, Fossen in Chapter 1 of [58] define guidance "the action of determining the course, attitude and speed of the vehicle, relative to some reference frame (usually the earth), to be followed by the vehicle". He also defines control as "the development and application to a vehicle of appropriate forces and moments for operating point control, tracking and stabilization". In Ruiz de Elviras thesis [156], the autopilots definition is expanded as the "criteria established to the adjustment parameters and/or constraints" to achieve a defined objective. Even if the autopilot concept is mainly used for the control system in charge of guidance, in a sailing boat, the course, attitude and speed of the boat depend on the combination of the different tasks carried out by the different crew members (steering, sail trimming, foils operation) onboard. This is the reason why a new definition of the autopilot concept will be used all along the thesis associated with the way the different systems are handled on board the real boat. When a system is meant to be handled by humans in the real boat, following the more general idea presented by Ruiz de Elvira in [156], the algorithm in charge of that system will be referred as an autopilot. If the system is controlled on board by other means than human sailors, the algorithm in charge of that system within the simulator will be referred as a control system. However, this definition is not clear for elements controlled by humans with a pseudo control-system assistance (mainly due to design rule restrictions regarding control systems on board). For the sake of practicality, these hybrid elements will be considered as control systems.

The autopilots or control systems will use different information, in order to achieve the predefined objective. For instance:

- The values of the boat variables to study such as position, velocities, etc (referred in [156] as space state variables).

- The values of the adjustment variables regarding all the adjustments that can be made on board to modify the boat behaviour and response (referred in [156] as adjustment state variables).

- The environmental conditions.

- Different performance and action sequences criteria related to the design goals and boat reality. 
This information will be processed by a certain algorithm in order to generate the optimum response towards the autopilot objective initially defined. For a more detailed description of the autopilot scheme implementation suitable for the time-domain VPP please refer to section 6.3.3 of [156].

One important aspect that has to be implemented inside the autopilots is the one related to how the adjustments can be made on board. For instance, the autopilot algorithm can change different adjustments when facing a specific situation but each one of them needs a certain time of operation until the adjustment is effective on board (trimming time, energy available on board, etc.). If the time of operation is not respected then the autopilot will be unable to reproduce the real boat behaviour. To model the time of operation of a certain element on board, Manuel Ruiz de Elvira introduced the rate of change of the adjustment in section 6.3.3 of [156], which is used to limit the amount of change of a certain parameter by time step.

The autopilots have to be adapted for each one of the elements suitable to be controlled on board. Each one of them will have a specific objective, adjustment parameters, rates of change and constraints. In fact, the autopilot will be different not only for each element but it has to be adapted for each situation it will be involved. For example, when analysing the exit of a tack, the objective (in this case maximizing boat VMG acceleration) will be different than when maximizing the VMG in a predefined course. This will also apply to the adjustment parameters, the constraints and the rates of change of these adjustments.

\subsubsection{Boat Elements}

For a foiling sailboat the following elements present on board can be suitable to be used with an autopilot or control system:

- Steering devices:

The principal steering device used onboard is the rudder and its associated adjustment variable is the rudder angle. When the autopilot is used to control this type of device, the objective will depend on the task to fulfil. When used in the dynamic analysis of certain manoeuvres, the objective can be as simple as maintain the boat heading, maximize VMG under certain environmental conditions, minimize the distance between two geographical waypoints, etc. For a simulation of a complete regatta, the autopilot has to be connected with an RMP module in combination with the autopilot objective (normally maximize VMG) to have enough strategic information to generate a realistic handling 
behaviour. This simulator autopilot is what is usually known as an autopilot in other industries.

- Sails:

Normally the objective is to generate the amount of driving force and heeling moment with the available righting moment to generate the fastest boat speed or the maximum acceleration. Other objectives for manoeuvres such as a tack can be minimizing the loss of distance to windward during the tack. The autopilot can use a simple de-powering scheme (like the one presented in [33]) as adjustment variables or if the aerodynamic sail model implemented is based on multiple trimming parameters as part of the model parametric space it can use such trimming parameters as adjustment variables.

- Foils:

Related to aircraft flight controls, the foils objective normally aims to maintain a certain ride height or a specific boat attitude to minimize dynamic instability and drag. The adjustment variables depend on the adjustment capabilities of the foils, the most complex systems nowadays allow to rotate the foils in three axes (cant, rake and toe) and one translation (foil up and down). The foils can be controlled by sailors or a control system, in fact, if the control system is based on mechanical devices using force inputs, such control systems can be modelled as special forces and moments models as explained in 3.3.3.

- Energy generation and management system:

The energy on board is normally limited and generated by human power (whose generation capacity decreases quickly with time and sustained load, a factor that has to be taken into account in the system model). The objective of the energy control system is to generate, manage and deliver the maximum amount needed in such a way that all the consumers on board (all the electric, hydraulic or pneumatic powered elements) receive what they need at any time, to fulfil specific operations, without consuming the total amount available, and knowing the energy generation capabilities (using the time operation concept introduced previously). If different operations have to be done at the same time and the energy available does not fulfil all the instantaneous requirements, the control system has to be prepared to deliver the energy to the element as soon as is available or to prioritize the delivery to a specific consumer based 
on its importance in the manoeuvre being made. The adjustment variables are normally related to the number of grinders available and how the energy is distributed to the different onboard systems.

- Crew position and moveable masses on board:

The position of the centre of gravity of the boat affects the amount of righting moment available and the boat's attitude. The objective with such elements is to maximize the righting moment available (to be able to generate more driving force with the sails) or to keep a certain boat attitude (for example to control pitch dynamics in the Moth foiler changing, the foils angle of attack leading to dynamic instabilities). The adjustment variables are the centre of gravity position over the deck for the crew or the quantity and the centre of gravity position inside the boat for a movable mass.

Each autopilot represents an added layer of complexity inside the VPP. In fact, each crew member (or following the simulator analogy each autopilot) communicates to each other in order to execute the task that he is assigned to. The main difficulty with autopilots is to allow the communication and interaction between each autopilot in order to obtain the best performance. This can be relatively easy when the objective for all the working autopilots for a specific simulation is the same. Otherwise, this can be addressed using a general autopilot with a weighted objective function taking into account all the objectives for each individual autopilot.

\subsubsection{Algorithms}

All the autopilots and control system algorithms are based on control theory, but an in-depth analysis of such topic is out of the scope of the thesis. An extensive research has been already done regarding marine vehicles control and guidance, such as the work made by Tristan Perez in ship motion control (course keeping and roll stabilization) in [131], or the work carried out by Fossen in his book "Handbook of Marine Craft Hydrodynamics and Motion Control" [59]. Other research has been done on specific marine vehicles, such as autonomous underwater robots like the one carried out by Somolinos in his thesis [162], or autonomous sailing boats like the one made by Cruz et al. in [34]. Different control algorithm options can be used and applied for the different autopilots and control systems, at least with the conventional displacement sailing boats, and some of them will be highlighted in the following list:

- Pre-introduced data: 
It is the simplest way to implement an autopilot but it is not considered as a control algorithm. It is based on a predefined time-domain set of actions, regarding the adjustment variables, to be performed along the simulation.

- Proportional-Integral-Derivative (PID) control algorithms:

The PID controller is a control loop feedback mechanism that can be applied to the different elements present onboard (mainly steering devices). Fossen in Chapter 12 of [59] explores the use in marine applications. Ruiz de Elvira in [156] explores their implementation in the time-domain VPP autopilots.

- Advanced Control algorithms:

As introduced by Fossen in Chapter 13 of [59], optimal and non-linear control algorithms and techniques can be used in marine applications. Among those advanced control algorithms, we can find linear quadratic optimal control, sliding mode control, state feedback linearization and integrator backstepping.

- Neural networks algorithms:

As part of intelligent control, the neural networks can be used to generate autopilots for the different systems onboard. To be able to use them, the neuronal networks that will handle the different elements have to be trained using a set of input-output data. This data describes the behaviour of the element to be controlled under the influence of a certain input. Priandana et al. in [144] present the usage of such type of control for a double propeller boat model.

It is important to note that foiling sailboats are dynamically closer to aeroplanes than to the conventional displacement sailing boats. This is the reason why autopilots and control systems for conventional boats have to be adapted for the dynamic problems of foiling boats, or other solutions have to be implemented. Foiling sailboats present a wider range of dynamic stability problems that have been already studied in aircraft. For example, the pitch stability in a foiling boat resembles an aircraft, the control system implemented for the aircraft to avoid this specific issue can be used for the same purpose in a foiling boat. Extensive research has been made regarding aircraft stability and control systems and is presented in a large number of publications: [49],[29], [163], [110]. 


\subsubsection{A General Approach for Neural-Network-Based Au- topilots}

The neural network can potentially be used for the different elements autopilots within the simulator. A similar implementation to the one proposed by Lewis et al. in [98] for robots can be adapted to mimic a specific sailor behaviour performing a specific task on board. A generalized approach based on a multilayer neural network controller combined with simulator generated input-output data obtained by repeatedly simulating manoeuvres and regattas in different situations during crew training is potentially the most general approach that can be implemented inside the simulator. Using the sailors that will sail the real boat to generate input-output data during the simulator training time allows obtaining good quality neural network training data (avoiding on the water measurement problems) that can potentially mimic implicitly the strategies and reflexes of the crew within the generated autopilots following the idea of modelling human performance using neural networks presented by Fix et al. in [54]. The generalized approach presented before uses an off-line learning procedure for an open-loop autopilot. An advanced version can be implemented when combining off-line preliminary learning with sailor simulator training generated input-output data and an on-line learning procedure combining neural networks with adaptive control theory in a closed loop scheme, introduced in section 1.3 of Lewis et al. [98] and developed later in the same work for robot applications, when the autopilots are being used.

\subsubsection{The Simulator as a Safe Test Environment for Control Systems}

Control systems are an important part in some of the new foiling sailboats, in fact, they are used to control onboard foils and other elements and systems. Real scale testing of such control systems is expensive and potentially dangerous for the material and the crew sailing the boat. The simulator not only uses the autopilots and control system models but it can also be inexpensive and a safe environment to test the behaviour of the different control systems present on board. In fact, it can be used in combination with other tools such as the genetic algorithms to optimize and finetune the control system parameters to improve its robustness and the overall boat performance. 


\subsubsection{Use of Autopilots to Improve Performance}

Autopilots allow making a wider use of the simulator even if the different tasks on board are not performed inside the simulator by a human user. In some situations, their use can also help to improve boat performance. Specific manoeuvres or regatta situations can be reproduced multiple times using intelligent neural-network based autopilots with an on-line learning scheme, like the one explained in 3.5.3 and time history data is generated. Then, the same data is collected when the sailors reproduce the same manoeuvres, training in the real boat or in the simulator. If the collected data shows different performance in comparison with data generated using autopilots, the time history of the different elements can be analysed to determine corrections to the way sailors are performing their specific tasks on board simpler.

Following the same idea, the sequence of actions (the order of the different actions made on board to face a manoeuvre or a regatta situation) towards a better performance can be determined using autopilots combined with Genetic Algorithms(GA). In fact, the sequence of actions can be modelled associating a different integer value for each action and generate with those integers a vector that will be the input parameter of the GA algorithms. The GA algorithms will iterate changing the order of the elements present in the vector (and therefore changing the sequence of actions) towards fulfilling the problem objective of maximizing performance. The performance objective will be expressed via a merit function that has to be determined to fit each manoeuvre or regatta situation.

\subsubsection{Unstable Boats}

As it will be explained later, the boat chosen for the thesis is the Moth foiler, a small dinghy equipped with foils and unstable behaviour. For the time-domain simulations presented in [18], a PID controller is used to maintain boat course. To avoid roll instabilities, in [18] the roll is fixed in order to allow a proper control of the PID in charge of the rudder. Following a similar approach, a specific heel angle control scheme using a PID controller is developed. In order to simplify the problem, the heading is locked during the PID tuning and testing stages. The numerical scheme used for the tests is a fourth order Runge-Kutta with a time step of 0.1 seconds, in order to avoid inducing large numerical errors in the simulation. The heel angle control scheme is based in two elements, a roll moment variation procedure using the elements available on board (the sail trim via the flat variable from the depowering 
model implemented, as described in Appendix D, ranging from 0.1 to 1 , and the crew position via the $\mathrm{Y}$ position variable of the crew centre of gravity referred to the sail boat centreline, ranging from 0 to 1.250 meters) and the PID. The PID controller calculates a control variable, ranging from -1 (overpowered situation) to 1 (underpowered situations), based on the heel error and managing the roll moment variation procedure. This procedure consists of:

- When the boat is underpowered (PID generated variable $<0$ ):

- If the sail trim parameter can be increased (not at its maximum value), increase its value until the PID variable requirement is fulfilled.

- If the sail trim parameter reaches its maximum value move the crew inboard until the PID variable requirement is fulfilled or the $\mathrm{Y}$ crew minimum position is reached.

- When the boat is overpowered (PID generated variable $>0$ ):

- If the sail trim parameter can be decreased (not at its minimum value), decrease its value until the PID variable requirement is fulfilled.

- If the sail trim parameter reaches its minimum value, move the crew outboard to increase righting moment until the PID variable requirement is fulfilled or the Y crew maximum position is reached.

Because the effects on roll moment of the sail trim parameter and the crew position are different (the flat variable affects more the roll moment than the crew position per unit change), the change rate on both variables is normalised in order to generate the same response per unit change in the transitional area every time the PID variable changes.

After implementing the heel control procedure, a PID tuning has been held. The main problem found when trying to obtain the different PID coefficients was related to the Moth inherent roll instability. In fact, the boat was not able to reach a stable state after a step change in the heel PID setpoint (target heel) or in the wind conditions (TWS), leading to a boat capsize. The traditional tuning methods were difficult to apply because of the large oscillations obtained and subsequent simulated boat capsize. Using a minimal step change (0.1 degrees of heel in the setpoint) with the Ziegler-Nichols tuning scheme combined the Pessen Integral Rule (as introduced by Pessen in [134]) allowed to determine the critical gain $(K u=0.25)$ and roughly estimate the corresponding period of sustained oscillation $(T u=7 s)$. This tuning 
procedure presented the best results among the traditional techniques when obtaining an initial PID coefficients estimation, that was later refined by manual fine-tuning to improve the PID behaviour. In order to obtain better results, a Genetic algorithm training scheme for the controller coefficients has been implemented using the Differential Evolution algorithm (a mono objective optimization algorithm presented by Storn et al. in [166]) applied to a PID setpoint (target) step change situation. The objective function, in this case, was the sum of the absolute value of the PID error each time step. In order to account for early capsizes, these situations were penalized using a high error value for each time step after the capsize, even if the total time-domain simulation time was not reached. Both techniques lead to similar coefficient results (the order of magnitude of the coefficients obtained is: $10^{-4}$ for $\mathrm{Kp}$, $10^{-4}$ for $\mathrm{Ki}, 10^{-1}$ for $\mathrm{Kd}$ ) and the heel PID controller allowed the simulated boat to maintain its behaviour for more than 30 seconds when it was not subject to changes in the external conditions. The PID controller managed to maintain the boat's heel during the first 18 seconds by changing flat and crew position variables in steps lower than $10^{-3}$ as it can be observed in table B.1. The last simulated seconds, the PID tried to correct larger changes in heel leading to an uncontrolled oscillation of the total moment around the X-axis, as it can be observed with more detail in table B.2. With larger values of the coefficients, the simulated boat crashed after a few seconds of simulation. When the PID controller with the determined coefficients was used in simulations where external conditions changed (a larger variable change rate is needed), it was not able to control the heel and avoid capsize. This suggests that in highly unstable boat situations, like the roll unstable behaviour of the Moth due to its lack of roll inertia, a simple PID controller is not a suitable option. In these situations, an adaptive method has to be used in order to adapt to the changing conditions, such as a PID combined with fuzzy logic or more advanced control systems based on stochastic control theory such as a Linear-Quadratic-Gaussian controller (a combination of a Kalman filter, a linear-quadratic estimator and a linear-quadratic regulator). In Table B.1 of Appendix B, the time history of the simulated Moth run using the heel PID controller described above and the force models (described in Appendix D) are shown with a time interval of one second. 


\subsection{A Note on Multi-Threading Execution and Multi- Computer Configuration}

The main goal for a practical simulator is to be able to calculate forces and moments models and to solve numerically the second order non-linear differential equations (3.2) in less time than the actual time step. When a high level of accuracy is needed (for instance being able to solve numerical schemes), a multi-threading approach has to be used. Being able to run all the models in multi-thread configuration creates the capacity to substantially reduce the execution time of an individual iteration. The main problem is related to the way the VPP is structured. For instance, in each time step, every forces model has to be calculated before the time-domain equation system will be solved. With an optimum configuration of one thread per model, the minimum execution time to calculate the models will be the time needed to calculate the slowest one. Some models depend on other models, like when a foil with a flap model has to be calculated after the model of the control system that manages the flap behaviour, reducing the value of using multi-threading. The reduction in computational time will be useful but limited (never divided by the number of threads available).

Nine tests were carried out to measure the time-saving improvement using multithreading when calculating with the Moth foiler models. The forces and moments models (described in Appendix D) were divided into blocks, containing each of the boat elements to be calculated, for instance:

- Block 1: Calculating the models related to the wand control system.

- Block 2: Calculating the models related to the rudder and its horizontal foil.

- Block 3: Calculating the models related to the hull.

- Block 4: Calculating the models related to Board and its horizontal foil.

- Block 5: Calculating the models related to the sail.

- Block 6: Calculating the models related to the windage.

- Block 7: Calculating the models related to the gravity forces.

- Block 8: Calculating the models related to hydrostatics.

Because of some model dependence (mainly between the wand control system and the board) different execution procedures were used: 
- Running the models in blocks.

- Running the models in tasks (based on model dependence):

- Task 1: Execution of block 1 and 4.

- Task 2: Execution of block 2.

- Task 3: Execution of the rest of the blocks.

The final tests configurations used are:

- Test 1: Running 1000 times each block using one independent loop per block. Using a thread for each block loop iteration in a thread pool architecture with a 4 threads processor with all the threads available. Waiting for each block loop to be finished to start the following one.

- Test $1^{*}$ : Running 1000 times each block using one independent loop per block. Using a thread for each block loop iteration in a thread pool architecture with a 4 threads processor with all the threads available. Executing all the loops at the same time.

- Test 2: Running 1000 times each block using one independent loop per block. Using a thread for each block loop iteration and waiting for each block loop iteration to be finished to start the following one.

- Test 3: Running 1000 times each task using one loop per task with a 4 threads processor with three threads available, one thread for each task. Waiting for each task loop to be finished to start the following one.

- Test $3^{*}$ : Running 1000 times each task using one loop per task with a 4 threads processor with three threads available, one thread for each task. Executing all the task loops at the same time.

- Test 4: Running 1000 times each task using one general loop for all the task with a 4 threads processor with three threads available, one thread for each task.

- Test 5: Running 1000 times each block using one independent loop per block without using multi-threading.

- Test 6: Running 1000 times each task using one loop per task without using multi-threading. 
- Test 7: Running 1000 times each block using one general loop for all the blocks without using multi-threading.

All the time measurements are presented in Appendix C. The most realistic situation taking into account other processor loads and model dependence is the configuration used in test $3^{*}$ presenting a time saving of approximatively $45 \%$ in comparison with the same configuration without using multi-threading which correspond configuration 6 .

When looking at the general picture, the simulator uses more elements than the actual time-domain VPP. It needs computational resources for the generation of motion cues for the Hexapod, the Engine managing virtual reality and visualisation and the acquisition system behind the user physical interface. In a scenario where even the execution of the core of the simulator (PASim) could present a lack of resources, a new approach has to be designed. Having the ability to use multiple dedicated computers to run each of the components individually seems the best solution. In order to ensure proper and fast communication the network protocol UDP described in [141] is implemented in each simulator component and modern network architecture has to be used (Gigabit Ethernet) in each computer and network hardware.

\subsection{An Example : The Moth Foiler}

When the idea behind the simulator was first started the boat candidate for testing the concept was chosen. Instead of choosing a more conventional boat, the approach was to select an apparently simple and little foiling boat but very complex dynamically and very unstable to really pushing the boundaries of the technology in existence. The chosen candidate was the Moth Foiler. 


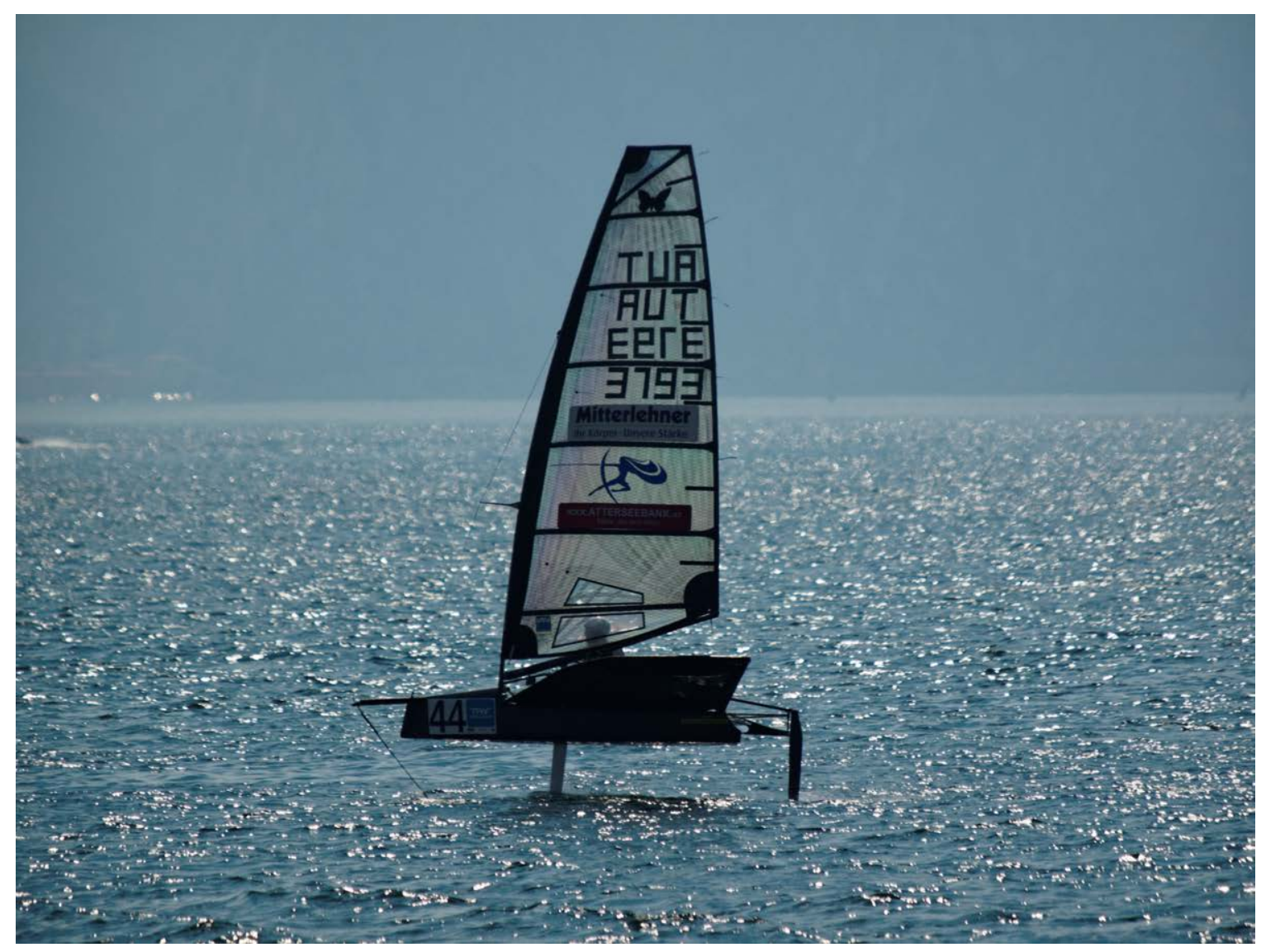

Figure 3.8: Moth sailing in foiling mode. Photograph by Manuel Ruiz de Elvira

\subsubsection{The Boat}

The International Moth Class is a boat class that manage the Moth foiler fleets. As specified in their website [4], the Moth is a sailing dinghy with the following general characteristics:

- Length overall: $3.355 \mathrm{~m}$.

- Beam: $2.250 \mathrm{~m}$.

- Max. luff length: $5.185 \mathrm{~m}$.

- Max. mast length: $6.250 \mathrm{~m}$.

- Hull weight: Unrestricted, general weight range 10-20kgs.

- Rigged Weight: as little as 26kgs. 
- Sail area: $8.25 \mathrm{~m}^{2}$.

- Restrictions: Multihulls, trapezes, moveable seats and sailboards are prohibited.

- Optimum skipper weight: 60-80kgs.

As explained in the class rule[3] number 1, "The International Moth is a singlehanded development class boat" with "open class rules". It is the result of many years of development since its initial conceptualization in 1928, that has included, across this history, the use of lightweight hulls and big sail area. Presently, it is one of the fastest single-handed monohull dinghies in production, capable of reaching more than 30 knots sailing supported on its foils. The class has contributed to the foiling boat's development since the 1990s as explained in [177] and now is one of the most active foiling boat classes in the world

The boat configuration consists of:

- A narrow carbon fibre hull with hiking wings.

- A carbon fibre mast.

- Only one mainsail.

- A daggerboard T foil with a flap controlled by a Wand system.

- A rudder $\mathrm{T}$ foil.

The boat could be observed sailing in foiling mode in figure 3.8.

\subsubsection{Forces and Moments Models}

The following forces and moments models try to represent the physics involved while sailing the Moth. All the details regarding those models are explained in Appendix D. The models are sorted by the type of element:

- Aerodynamic Models

- Sails

* Bögle et al. [18] Aerodynamic Model based on North Sails Flow data combined with Heikki Hansen work [72] sails coefficient curves modified to include the last IMS flat and associated twist function depowering scheme. (Mixed model) 
* Aerodynamic model based on the TFWT (University of Auckland) data collected by the author. (Interpolated Model)

* IMS model. (Analytical model)

- Windage

* General model based on Bögle et al. [18] work with improvements and corrections (For rig, crew, hull and emerged appendages below the hull). (Analytical model)

- Hydrodynamic Models

- Lifting Surfaces (Daggerboard and Rudder T-foils)

* Elliptical distribution lifting line. (Analytical model)

* Complex Lifting Line. (Numerical model calculated in execution time)

* VLM. (Numerical model calculated in execution time)

* End-plating and Piercing corrections. (Analytical model)

- Hull

* Hull model based on raw data from Beaver et al. paper [10] (with hull hydrostatics incorporated). (Interpolated Model)

- Other Models

- Control Models

* Improved and completed version of Bögle et al. [18] Wand control model, using a Newton numerical scheme for finding equilibrium of the Wand system forces and moments. Normal or paddled wand version with different level of complexity available. (Analytical model)

- Other hydrostatics and weight stability models

* Yacht and crew weight model (with crew optimization). (Analytical model)

* Appendage hydrostatic forces model based on immersed volumes and centres calculation. (Analytical model)

The following selection of the models presented above was used to generate the polar curve for the boat of Figure 3.9 and during Moth time-domain simulations such as the ones presented in Appendix B: 
- Bögle et al. [18] Aerodynamic Model modified to include the last IMS flat and associated twist function de-powering scheme.

- Improved and corrected Windage model based on Bögle et al. [18] work.

- Complex Lifting Line with End-plating and Piercing corrections for all the lifting surfaces.

- Hull forces model based on raw data from Beaver et al. paper [10].

- Improved and completed version of Bögle et al. [18] wand control model.

- Yacht and crew weight model (with crew optimization).

- Appendage hydrostatic forces model based on immersed volumes and centres calculation. 


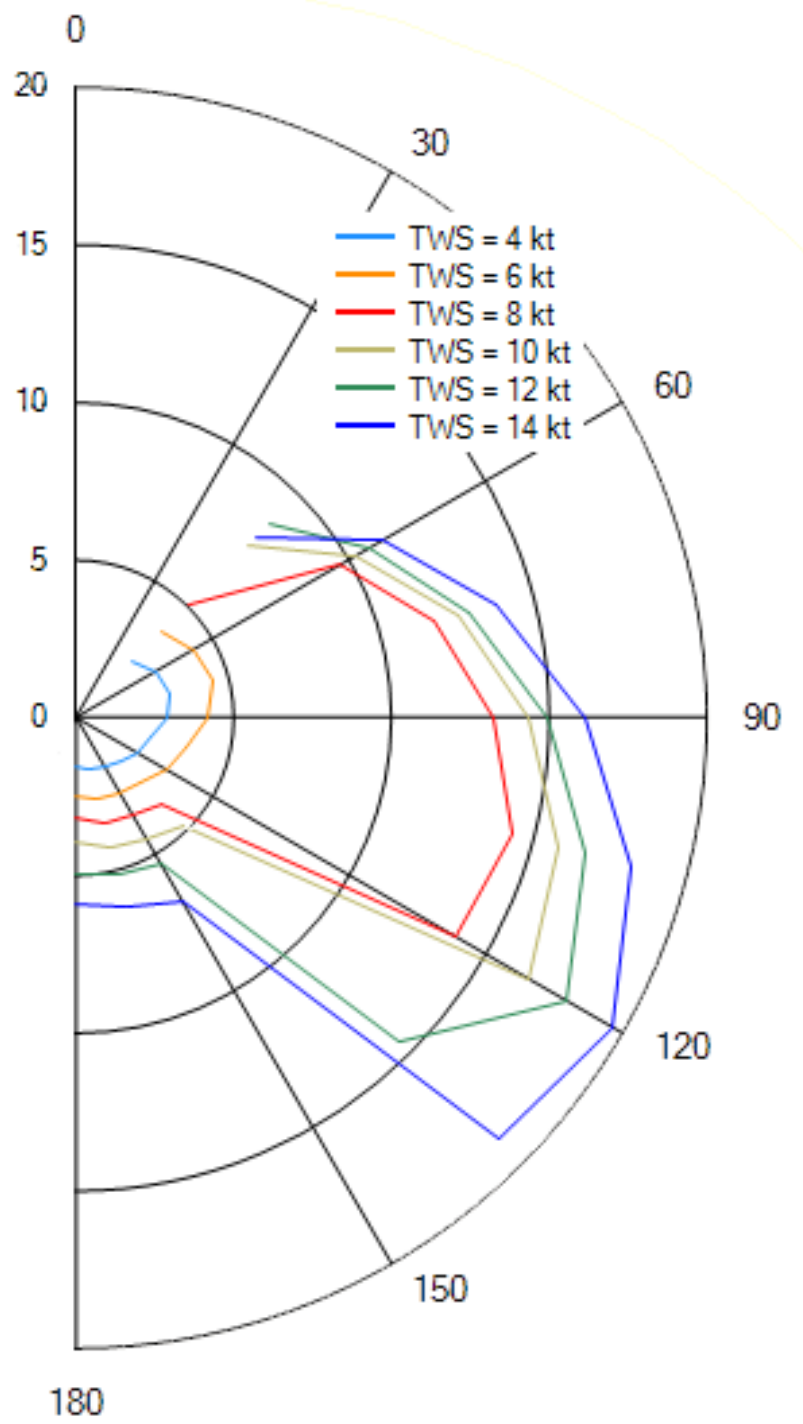

Figure 3.9: Moth polar curve generated using PASim.

\subsubsection{An Example of the Foiling Moth Simulator Usage: The Transition Study From Non-Foiling to Foiling}

The simulator can be used to study specific dynamic situations that will affect foiling boat performance on the water. For example, the simulator was used to study the acceleration capability of the foiling Moth while transitioning from a non-foiling to a foiling situation caused by an increase in the wind speed.

The time-step used in this simulation was 0.1 seconds combined with the midpoint numerical scheme. The initial values were obtained from a pre-calculated equilibrium 
condition of the boat sailing in 6.7 knots of TWS, a TWA of 90 degrees and 4.892 knots of BSP with the yacht sailing in displacement mode. A gust was generated changing the TWS from 6.7 knots 10 knots during the first 6 seconds of the simulation, maintaining the higher wind speed after that. In order to maintain the heading and the heel angle, a set of settings (rudder angle, crew position and flat sail trim setting) were adjusted in every time-step to maintain the total moments around the X-axis (Mxx) and Z-axis (Mzz) equal to zero. In order to help the transition, the crew $\mathrm{X}$ position was moved from the initial equilibrium position near the centre of the hiking wings to the rear of those wings, correcting the boat pitch angle. The crew Y position and flat sail trim variable were used to correct Mxx. The rudder angle was used to correct Mzz. The total simulated time was 35 seconds.

As it can be observed in Figure 3.10 the boat was able to accelerate from 4.892 knots to 12.686 knots of BSP after 35 seconds, foiling after 16 seconds at 8.949 knots of BSP. The wand system was able to maintain the boat height close to 0.55 meters above the water after taking off (Figure 3.11). Repeating this simulation with different parameters of the wand control system can be used to evaluate its effect on the transition. The boat pitch angle increased during the transition to reach a new position after the transition.

This is just a simplified example to show how this type of study can be used to optimize crew movements and onboard adjustments to refine the athletes technique using all the simulator resources available (visual cues, kinematic cues, etc) for an easier and more efficient training. However, it is important to note that despite the simplicity of this simulation, it involves some not-obvious considerations such as the fact that the movement of the crew causes a change in the yachts inertias (with the crew representing $2 / 3$ of the total weight) and the generation of a force on the yacht by the crew to make that movement happen.

The data of the complete simulation (in time-steps of 1 second) can be found in Table B.3 Appendix B. 


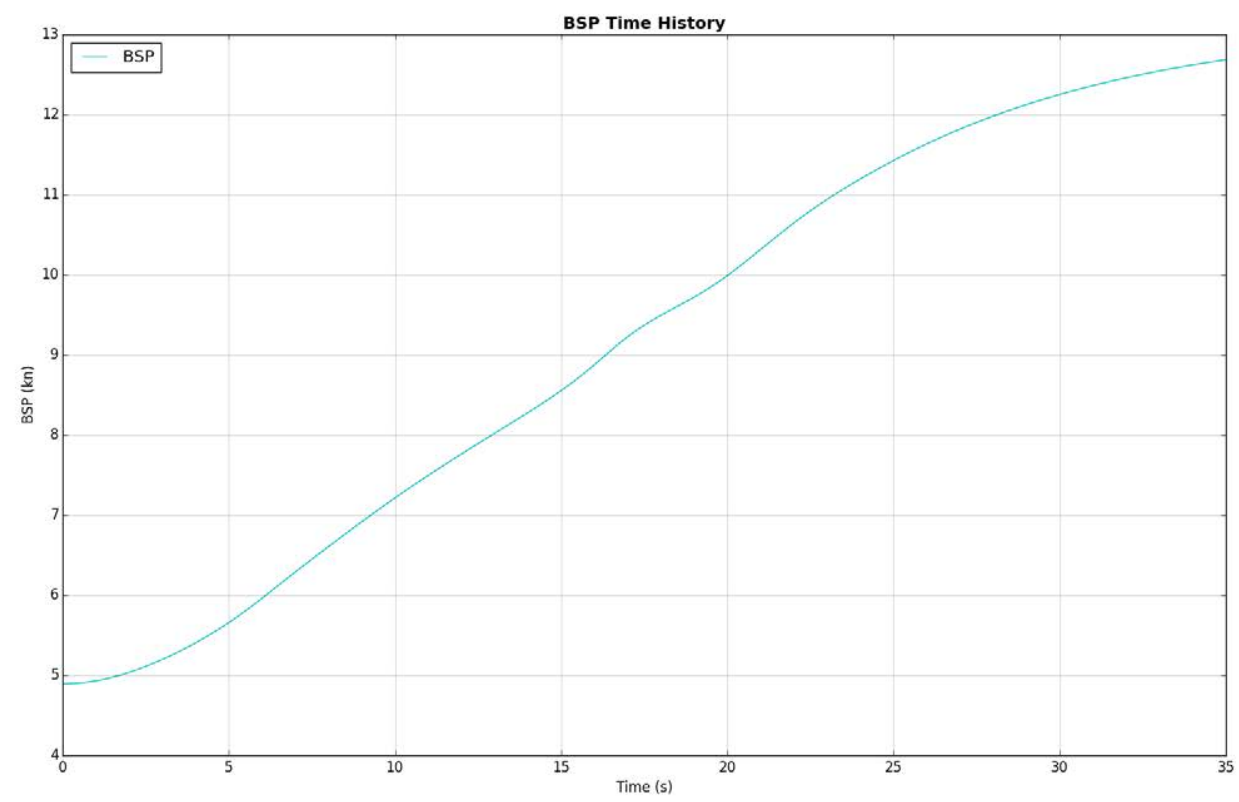

Figure 3.10: BSP time history of the Moth foiling transition.

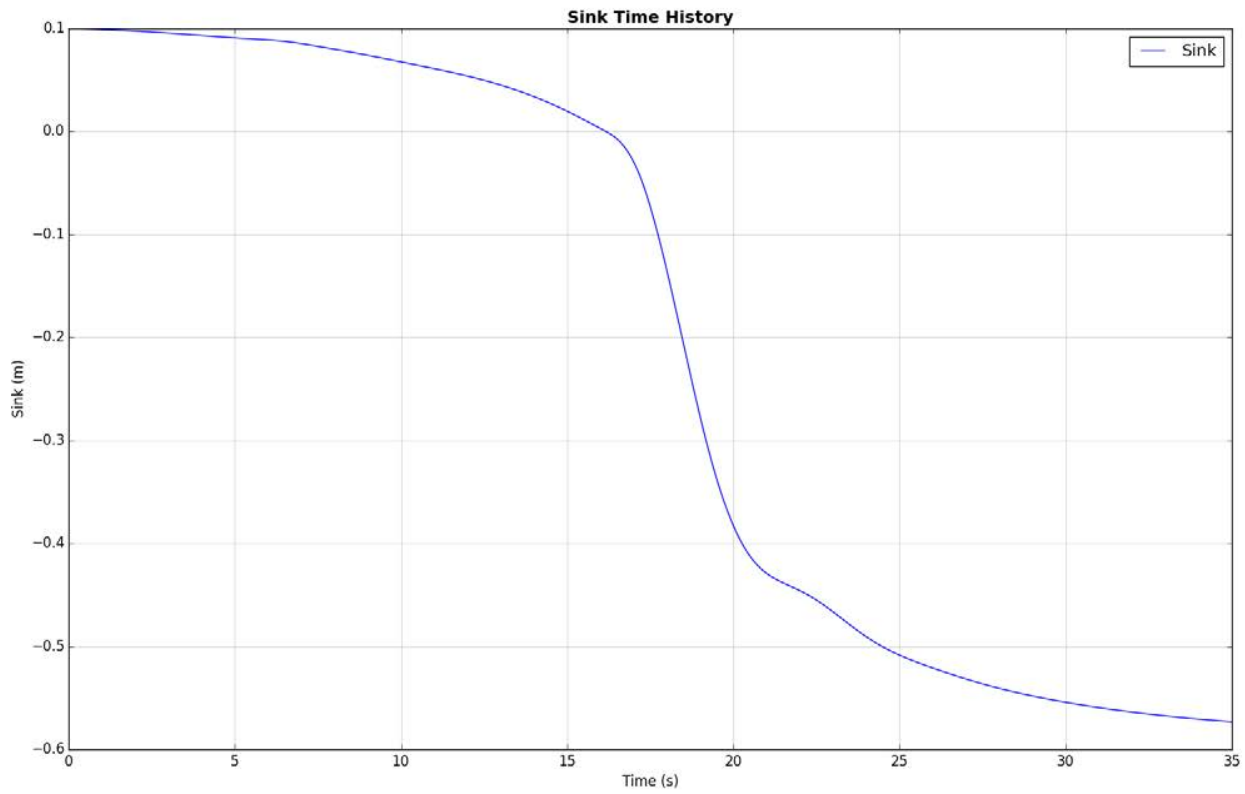

Figure 3.11: Sink time history of the Moth foiling transition. 


\section{Chapter 4}

\section{The Motion Platform}

As explained in chapter 1 , the simulator is a combination of different components in charge of reproducing specific aspects of the physics behind sailing. The component in charge of reproducing the physical sensations related to movement or acceleration is the motion platform. This platform will be different depending on the degrees of freedom needed for each type of boat. The simulator as a general tool has to be able to calculate all the cues needed for every configuration or at least the boat variables needed for it. For a general overview of the motion platform implementation within the simulator please refer to the diagram in Figure 2.1. For a specific overview of this module implementation please refer to the diagram in Figure 4.1. All the elements within this module will be described in the following sections. 


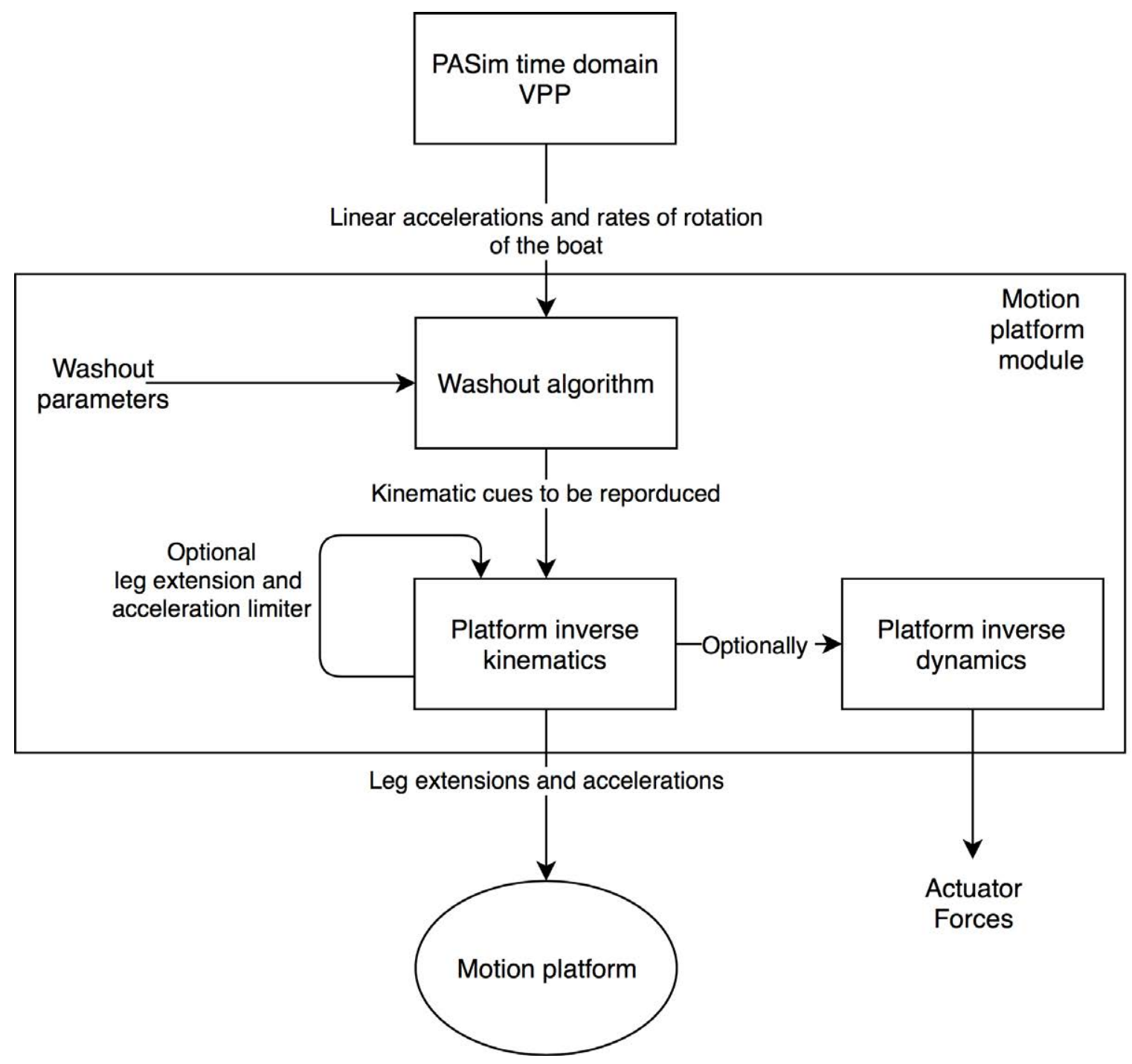

Figure 4.1: Motion platform module block diagram

\subsection{The Degrees of Freedom}

The first aspect that will determine the number of kinematic cues to be reproduced is the type of boat that will be simulated.

\subsubsection{Conventional Monohulls and Slow Multihulls}

As explained in chapter 3, even if the time-domain VPP could carry up to 6 DOF calculations, for slower boats the reality could be reproduced with no more than 3 or 4 degrees of freedom (ORC VPP uses 2.5 degrees of freedom with successful boat performance prediction [33]). In fact, when trying to simulate such situations, in the past, only one kinematic cue was reproduced (at least in more or less calm sea 
conditions, because waves could induce boat movements and accelerations). This approach was used for the first commercial simulator, the VS-1, presented in [12]. In this case, a real dinghy hull was mounted into a specific rig able to reproduce the heel angle motion. If waves are taken into account or a more realistic reproduction is desired, a rig capable of reproducing heel and pitch motion could be used. A full 6-DOF approach could be useful for such boats and was used in past monohull America's Cup campaigns. In fact, when a fully dynamic situation has to be studied (for example how the boat will accelerate after some situations) a 6-DOF approach will be the option to choose. Normally, the number of kinematic cues reproduced has to be equal or lower than the DOF calculated by the VPP. In fact, even if a 6-DOF approach is used, the number of kinematic cues reproduced are related to the dynamic characteristics of the boat and the goal of the study carried out with the simulator (sometimes simpler rigs could be used). However, for fast planing boats such as the $49^{\text {er }}$ dinghy (observed sailing in figure 4.2) a full 6-DOF/6-DOF approach could be preferable (using the sailboat 6-DOF for the calculations within the VPP and a platform capable of reproducing 6-DOF of motion). For slow multihulls (like big cruising catamarans) the same principles declared for monohulls have to be applied.

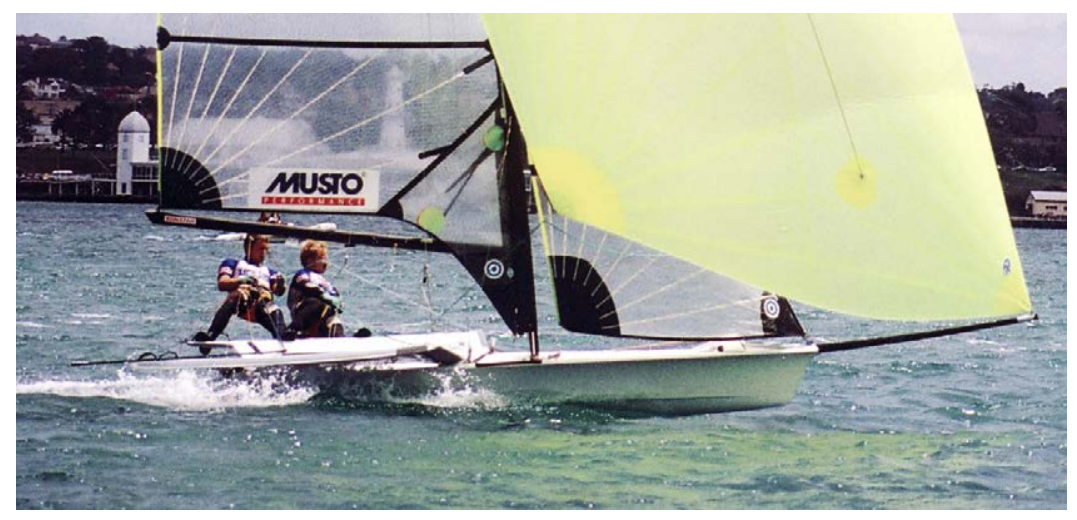

Figure 4.2: $49^{\text {er }}$ dinghy sailing.

Photograph by local Geelong yachtsman.

Source: https://commons.wikimedia.org/wiki/File:

49er_skiff_sailing_AUS_nationals_Geelong.jpg

\subsubsection{Fast Multihulls and Foiling Boats}

When dealing with fast boats, which sail by generating high AWS with low AWA (generally fast multihulls and foiling boats), fewer options are available. The sailors of these kinds of boats are used to sailing the boat according to their own trained way to feel the boat's physical behaviour. Proper reproduction of every kinematic 
source available is needed to be sensed by the simulator user. Different solutions could be adopted to reproduce the boat motions. Even if the usual solution is to use a Stewart 6-DOF parallel robot (Hexapod) [164], special configurations could be developed when a specific degree of freedom is out of the motion and acceleration range of the platform or it is predominant over the others (motion platforms 6-DOF expanded approach). For example, in sailboats heel could be considered separately because of its higher amplitude range. Special prototypes have been developed to face this kind of problem, like using a combination of a Hexapod moving over a linear rail (such as the Mercedes-Benz Driving Simulator [113]) or the special cabledriven parallel robot developed by Fraunhofer IPA and Max Planck Institute for Biological Cybernetics (MPI) [106], where highly dynamic simulations with complex acceleration patterns could be reproduced. For these kinds of boats (like the Moth Foiler, the AC50 or displacement catamarans such as the AC45 version without foils presented in figure 4.3) the 6-DOF/6-DOF approach (using the sailboat 6-DOF for the time-domain calculations inside the VPP combined with a platform capable of reproducing 6-DOF of motion) or 6-DOF/6-DOF expanded configuration approach should be used.

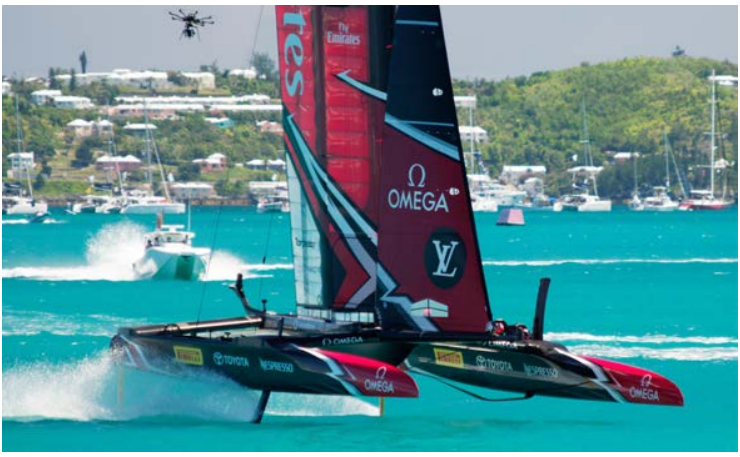

(a)

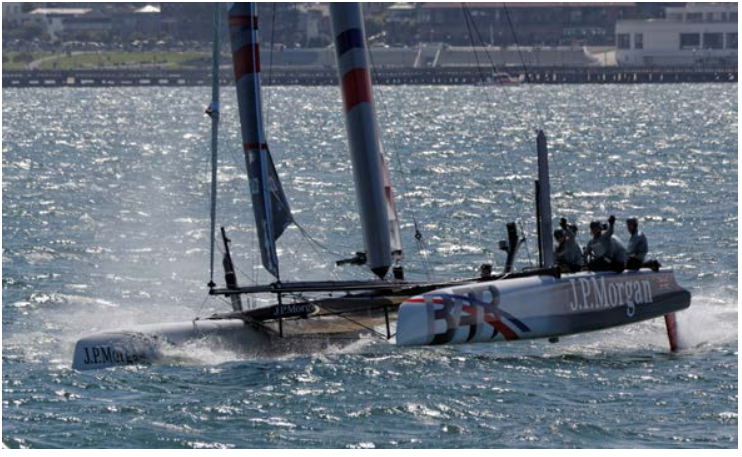

(b)

Figure 4.3: AC50 (a) and AC45 (b) sailing.

Photographs by Manuel Ruiz de Elvira

\subsection{Reproduction of Motion and Position Versus Reproduction of Rotational Velocities and Lin- ear Accelerations}

Other considerations have to be taken into account, to determine which type of kinematic motions will be reproduced. Two different philosophies could be used, to reproduce the full motion (which implies also the reproduction of the acceleration) or 
to reproduce linear accelerations and rotation velocities. It will depend mainly on the number of DOF to reproduce with a specific technology and also on how the visualisation system will be mounted and used.

\subsubsection{A Tool to Reproduce Motions and Position}

When a low number of kinematic cues have to be reproduced (one or two cues) simpler rigs could be used. For instance, a frame combined with one or two electric linear actuators could be enough to reproduce the boat exact angular motion and position for heel and pitch (in a boat sailing in normal circumstances) without reaching the rig angular limits. This setup is similar to the ones used in [12] and [116]. When the calculated boat motion is reproduced, after evaluating the feedback from experienced sailors, Mulder et al. in [117] concluded that the most engaging motion to contribute the sense of presence for conventional boats is the roll motion and the pitch motion (but only when combined with the roll motion). During Mulder's et al. study, other motions, such as heave, degraded the sense of presence. This can be related to the fact that the visualization system is not offering the level of realism needed (the screen is not large enough or the motion cues do not follow the graphics) or the VPP models do not generate the proper cues. In fact, when the visualization system is mounted outside the moving platform (for example using screens fixed to the wall instead to the platform in charge of reproducing the kinematic cues like in [117]) it is important to be able to reproduce the motion precisely following the graphics movement. If the user is able to identify the visualization system as something uncoupled from the platform, he has more visual references that help him to know exactly if the motion system is acting in a proper way. If the platform is able to reproduce a large number of motion cues, the user looking at the screen will be more aware of moving differently than the fixed screen, degrading the sense of presence. In foiling and fast boats, sensing boat behaviour is a vital part when handling the boat. In fact, it is important not only to the helmsman but also to the crew that does not have a visual reference of the boat moving (for example a trimmer looking to the sail). In this case, kinematic cues have to be reproduced for the 6-DOF and other techniques have to be used to avoid that presence degradation.

Motion and position reproduction should be used for some specific tasks when using the Simulator. One such task, described in section 2.2.6, is testing deck gear and deck layout configurations. With the one-to-one scale deck mock-up mounted on top of the 6-DOF platform, the skipper and the engineers could test the gear layout while moving the platform to specific sailing positions (could be obtained 
by calculating VPP equilibrium solutions) or reproducing predefined accelerations patterns (generated by specific time-domain analysis with the VPP) cyclically.

\subsubsection{A Tool to Reproduce Rotational Velocities and Linear Accelerations}

To overcome the problem previously presented, while trying to reproduce a big number of motion cues, a different approach has to be used. In fact, it has been present in the aeronautic simulation industry for years (used in Full-Flight Simulators (FFS) for pilot training). The approach consists of mounting the visual system inside a closed space on top of the simulator to isolate the user from the outside. Being isolated prevents the user from taking spatial references external to the simulator. In vehicles with closed cockpits the problem is easily solved. For example, for jetliners, a reproduction of the nose of the plane mounted on top of the platform with projected images in the plane's windows will be enough. For open cockpits configurations, which is the case with sailing boats, the task becomes more complicated and a wide range of view has to be covered (in fact, for an ideal experience the whole 360-degree range of view has to be represented). The full range of view could be covered using Virtual Reality headset, but such devices have their own associated problems as will be described in the following chapter.

As explained by Dichgans et al. in [46], self-motion perception is mainly related to the interaction of the human visual and vestibular systems subjected to visual stimulations, rotational velocities and linear accelerations. Harris et al. in [75] explain that linear acceleration can be also be sensed by:

- Specialised visceral graviceptors (that can be found specifically in the region of the kidney) but with a poor directional estimate. Such receptors will be stimulated at the same time as the vestibular system while using motion cue generation. Because the motion platform will stimulate the whole body at the same time the effects of these receptors are considered included in the vestibular system.

- The somatosensory system via pressure and stretch detection on the skin and in muscles, joints and visceral organs (for example when a subject is seated and undergoing vertical acceleration, the body parts in contact with the seat will have the cutaneous receptor stimulated because of the forces generated). Such perceptions are located in specific body parts and could be stimulated in absence of motion by exerting pressure over certain areas. Such methodology 
could be useful for seated handling situations (for example in planes) but not for the usual sailing boat handling positions.

They also explain that self-motion perception could be sensed by the so-called proprioceptors (some of the systems and receptors described above could be included inside), but the proprioceptive system is normally in charge of determining relative movement of body parts. Like the somatosensory system, this system will not be specifically stimulated.

If we consider the simulator as a global self-motion perception problem (where the goal is moving the whole user body), then the solution seems to be to generate a combination of visual and vestibular system stimulations. The visual stimulus should be reproduced using a realistic visualization system and rotational velocities and linear acceleration should be reproduced using a physical device in a full 6-DOF or extended 6-DOF configuration (using a Stewart 6-DOF parallel robot or a combination of the same robot with linear rails or angular movement devices). Instead of developing a specific approach with specific body parts stimulation (that could be used in a seated simulator handling situation), the approach proposed for this thesis will be to stimulate the whole body at the same time, allowing the reproduction of multiple types of boats using the same simulator configuration with very few changes. This is the same philosophy adopted in the time-domain VPP.

Within the simulation industry there is discussion regarding whether or not it is necessary to generate all the stimulations described previously or if a good amount of realism can be achieved using only the simulator visualization system. BürkiCohen et al., in [23], discuss the problems associated with the usage of a Stewart platform for vestibular cueing and explain the limited reliability of the vestibular system (loss of sensitivity after repeated stimulation and spatial disorientation after unusual motion cues). They propose the use of other motion cues than the visual and vestibular ones (the proprioceptive and tactile stimulations). They also present and compare other advanced motion simulation technologies such as the NASA Vertical Motion Simulator (VMS), the centrifuge motion simulator - Wyle's Dynamic Flight Simulator (WDF), the Desdemona Simulator combining a 6-DOF platform with a centrifugal design or even the In-flight Simulator using actual aeroplanes to mimic other aeroplanes. Such systems try to overcome the limited capability of the Stewart platform when generating sustained acceleration, following the same concept as the already mentioned 6-DOF expanded configuration. The selection of a system based 
on a 6-DOF motion platform implies a high economic cost, a cost-saving alternative is explored in [23], the dynamic seat which is able of generating some vestibular, proprioceptive and tactile motion cues in a fixed-based trainer. These solutions are oriented for aeroplane simulation but motion patterns in boats are different from the patterns in aeroplanes. The cockpit layout and user position in a sailing boat are not suitable to solutions like the dynamic seat so more conventional approaches such as the Stewart platform, where the user has more freedom to move, are preferable.

Other problems (not associated with the concept of presence and realism) are involved. For example, as explained by Howard in [78], the visual, vestibular and proprioceptive information received by humans help to solve ambiguities when dealing with an orientation and self-motion situation. McCauley et al. in [109] explain that such ambiguities could be created by the Virtual Environments when generating visual, but not vestibular, stimulation. Welch, in [187] and [188], explains that the human perception system is able to adapt itself and compensate for changing conditions. Such stimulation ambiguities could also trigger motion sickness instead of adaptation.

Following a general approach, the configuration of an integrated visualization system combined with a 6-DOF motion platform is the solution chosen for this thesis. More precisely the physical platform used and implemented in the simulator will be a modern, electric actuated version (cheaper in maintenance and more reliable than the hydraulic implementation) of the 6-DOF parallel robot known as the Stewart platform. To overcome the inherent limitations of such a configuration a washout filtering system is also implemented and will be explained later. As a modular system, when using the simulator, motion cues could be deactivated if needed (when a motion system is not in use because of economic reasons or due to lack of space). Alternative motion cue generation algorithms could be implemented to fit other motion systems like the 6-DOF expanded configuration.

\subsection{The Stewart Platform: A Six Degrees of Free- dom Parallel Robot}

The motion platform selected as the base case in the thesis is the Stewart platform manipulator. A brief description of such a manipulator will be developed and the chosen inverse kinematic and dynamic algorithm will be described qualitatively. 


\subsubsection{Description}

The main solution adopted in the state of the art simulators (at least outside the development of experimental simulators) is the 6-DOF parallel robot (Hexapod) known as the Stewart (or Stewart/Gough) manipulator (or platform). As comprehensively described by Dasgupta et al. in their platform review [41], the Stewart platform appears for the first time in Stewart's paper [164], where a 6-DOF device was used to simulate flight conditions. The device presented in this paper had a different geometry than the one we know today (a triangular upper platform combined with three adjustable legs combined with two-axis joints). In fact, Gough proposed the use of a fully parallel approach using a combination of six linear actuators (closer to the configuration of the tyre testing machine presented by Gough et al. in [67]).

Far from the initial designs and proposals, presently the generalized Stewart platform or manipulator is composed of two rigid bodies (the platform and the base), six linear actuators with two joints each, attaching each actuator to the base and the platform. It could be found in two main joints configurations:

- The 6-SPS (spherical-prismatic-spherical) configuration (Figure 4.4a) using:

- A spherical joint to attach the lower part of the actuator to the base.

- A spherical joint to attach the upper part of the actuator to the platform.

- The 6-UPS (universal-prismatic-spherical) configuration using (Figure 4.4b):

- A universal joint to attach the lower part of the actuator to the base.

- A spherical joint to attach the upper part of the actuator to the platform.

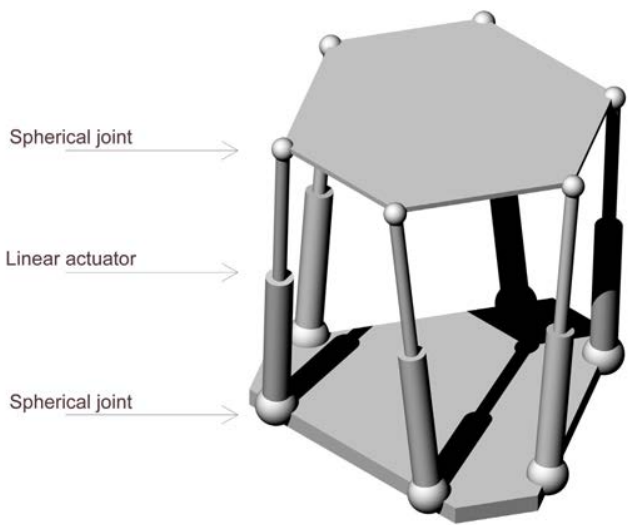

(a)

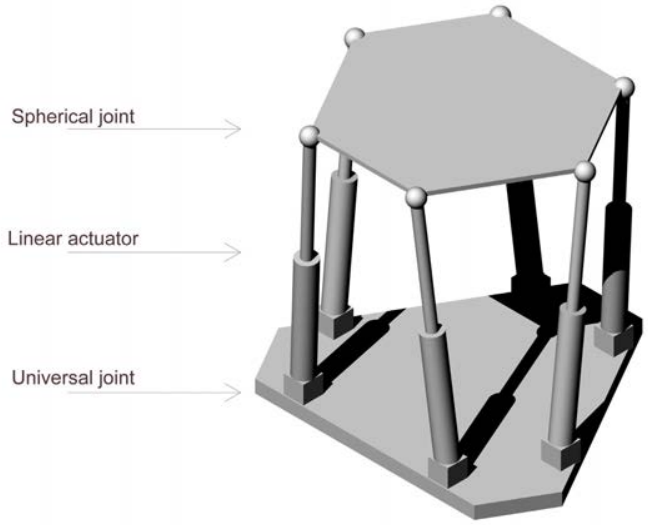

(b)

Figure 4.4: 6-SPS (a) and 6-UPS (b) platforms 
All the configurations present the same behaviour regarding the input/output results. The 6-SPS also presents a passive DOF in each leg related to the rotation about their own axis.

The Stewart platform is the subject of intensive research, not only in the simulator industry but also in general robotic applications, since Hunt's book [79] publication where he suggested the advantages of such devices in comparison with conventional serial robots. In fact, the first Stewart platform developed for other purposes than the simulator use was presented by McCallion et al. in [108], including some theoretical and numerical analysis regarding mobility and direct kinematics. Fitcher et al. in [53] presented a solution for inverse position kinematics of each individual leg using the conventional method of serial manipulators with the Stewart platform. Fitcher derived in [52] the kinematic equations of the general Stewart platform and also presented the first simplified dynamic formulation (using legs without mass and joints without friction) and the singularity condition. Merlet in [114] dealt with the solutions of kinematic equations, developed the Jacobian and derived more general dynamic equations.

For further information please refer to Dasgupta et al. review [41].

\subsubsection{Motion Cue Generation and Analysis Algorithms}

Since its first introduction in [164], a big effort was made to be able to move and control the Stewart platform using computer software and communication interfaces. In the following sections the algorithms used for the platform kinematics and dynamics implemented in the Simulator will be described qualitatively. The platform kinematics problem can be solved in two different ways. The first one is when the platform position is known and the leg extension has to be determined (inverse kinematics problem). The second one is when the 6 leg extensions are known and the platform position has to be determined (direct kinematics problem). All the algorithms described qualitatively in the following section were selected for their implementation simplicity and performance. The platform configuration chosen for the Simulator was the 6-UPS configuration because its simplicity compared to the 6-SPS configuration (no passive degrees of freedom in the actuators). 


\subsubsection{Inverse Kinematics}

The simulator motion cue generation can be observed as a simple inverse kinematics of the Stewart Platform. Knowing the position of the Simulator platform, which is related to the rotation velocities and linear acceleration calculated by the timedomain VPP, the cues needed to move the platform to the desired position will be calculated using inverse kinematics. The implemented algorithm for the inverse kinematics is the one presented by Dasgupta et al. in section 2.1 of [40], being reasonably efficient and computationally inexpensive (non-iterative algorithm based on basic matrix operations). This implementation calculates leg extension, leg sliding velocity and leg sliding acceleration, information normally needed by the actuators (at least extension and sliding acceleration). A block diagram of the implemented algorithm is presented in Figure 4.5.

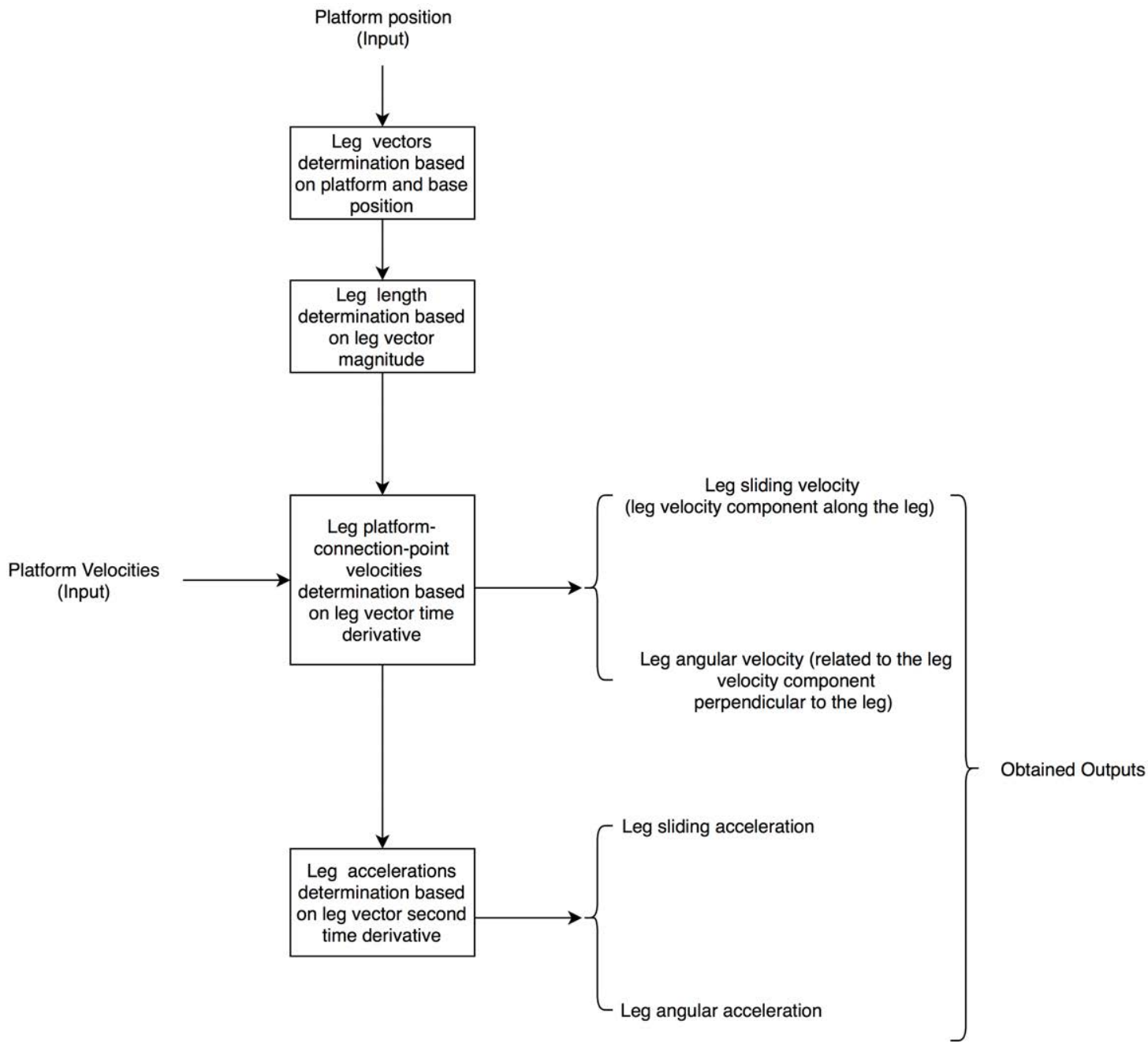

Figure 4.5: Block diagram of the Inverse Kinematics implementation. 


\subsubsection{Direct Kinematics}

From the motion platform cues generation point of view, since the position of the platform is known, the inverse kinematics are enough to move the platform. But to avoid reaching the platform limits in actuator length, velocity and acceleration, a limiting algorithm that correct the platform cues to operate within the platform limits needs to be used. Because the limiting algorithm checks and modifies directly the cues generated by the motion algorithm, a direct kinematic implementation has to be used to know the platform exact position that will be used for further calculations. The direct kinematics allows calculating the platform position when the actuator lengths are known.

These methods can lead to up to 40 solutions (complex or real) platform positions for the same leg extension configuration as explained in section 6 of [41]. Different numerical schemes are presented in section 6.2 of [41], allowing to find the possible real platform position solutions. In order to avoid the problem described, the following comparison scheme is proposed in this thesis. The different solutions are compared to the last known position of the platform in the last time step of the motion algorithm. The selected solution is the one that is the closest to that known position (present the lowest error in the 6 degrees of freedom of the platform). This solution is selected and set as the next platform position to be used in further calculations within the platform motion algorithm. For a more accurate selection, inclinometers and accelerometers can be installed to deduce the platform angles in order to compare if the selected solution will match the measured platform angles.

One example of the limiting algorithm previously explained is the implementation made by Raid and Nahon in [150], [151] and [151], included in the platform cue generation part of their washout algorithms.

\subsubsection{Inverse Dynamics}

The inverse dynamics method to determine the platform dynamics consists in calculating the upper platform dynamics based on the platform accelerations and inertia to be able to determine the force in each leg. Even if the dynamics are not directly involved in the motion cue generation, it is a valuable tool to verify the validity of a certain configuration. In fact, using inverse dynamics when knowing the characteristics of a certain Stewart platform and the payload, help to assess if a certain platform solution will comply with the needs of the motion simulation regarding leg 
forces and acceleration. The implementation chosen was the Newton-Euler formulation approach for the inverse dynamics presented by Dasgupta et al. in [40] for a 6-UPS Stewart Platform. The formulation for the 6-SPS is presented by Dasgupta et al. in [39]. This implementation divides the problem into two parts:

- Leg dynamics - described in section 2.2 and 2.3 of [40], allowing the determination of the leg forces used in platform selection and validation assessment.

- Platform dynamics - described in section 3 of [40], mainly used for the mechanical design.

For initial platform selection and validation purposes, the leg forces could be estimated using motion cues generated by a time-domain calculation with the VPP (a pre-recorded virtual regatta or an extreme sailing situation reproduced using the time-domain VPP) or with real sailing information collected during on-the-water boat testing.

This Newton-Euler formulation was selected because of its simplicity and also because of its reasonably inexpensive computational cost. It runs in execution time along with all time-domain calculations, washout and cue generation, if needed. A block diagram of the implemented algorithm is presented in Figure 4.6. Other implementations and methodologies can be found in section 11 of [41]. 


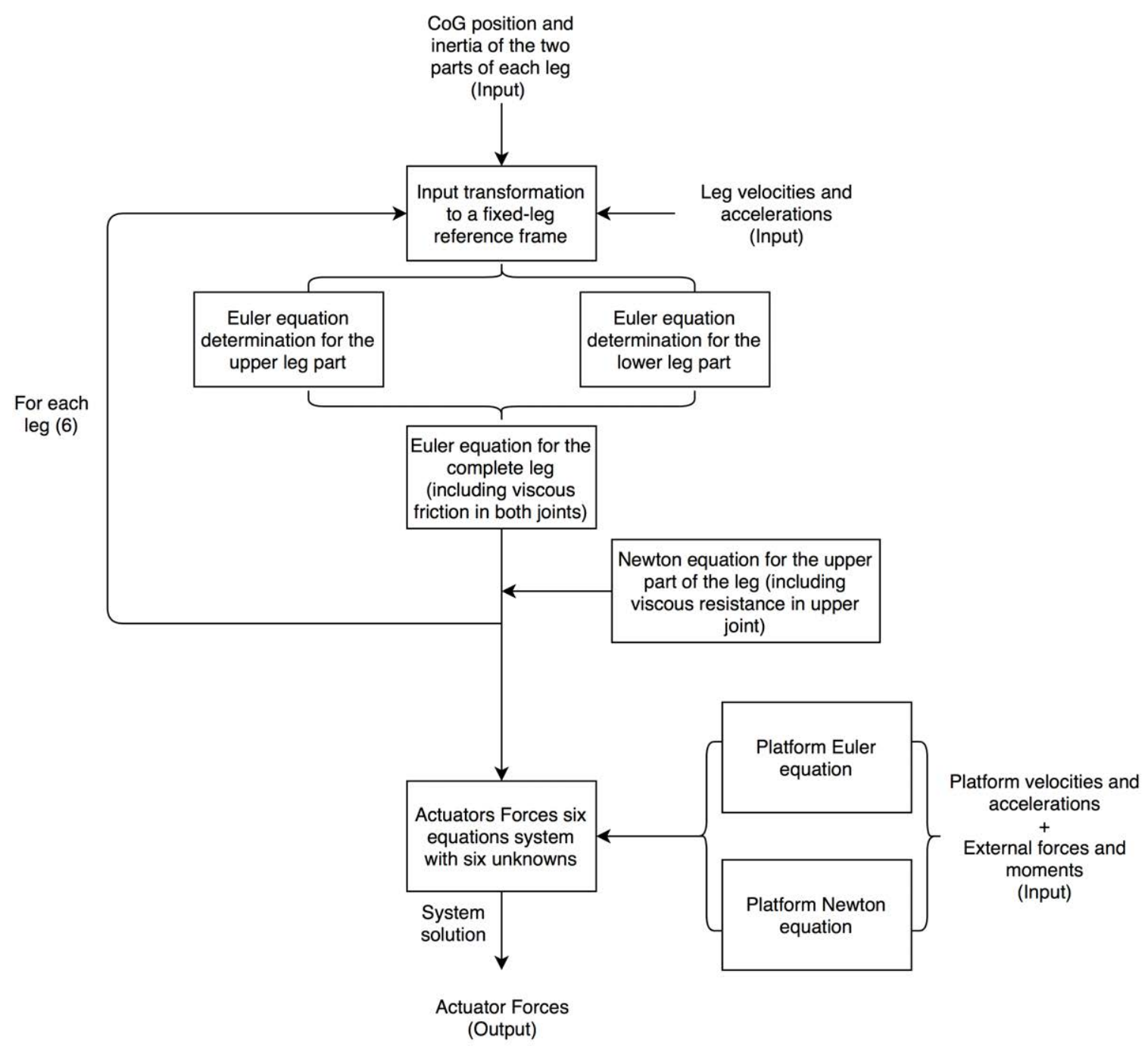

Figure 4.6: Block diagram of the Inverse Dynamics implementation.

\subsubsection{Limitations}

As explained in [23], the use of the Stewart platform for motion reproduction in a Simulator presents specific limitations. Some of them are already solved by means of faster computers allowing a better synchronization between the motion cues and the other cues generated by the simulator. Other limitations are purely related to the geometrical and physical characteristics of the platform. The actuators used in the Stewart manipulator present a limited capability of linear displacement which implies that the motion cues are constrained within the limits of the actuators, both in duration and amplitude. This could lead to an improper representation of the rotational velocities and linear accelerations calculated by the VPP. One of the solutions to this problem is to use specific algorithms called "washout", allowing the 
generation of proper motion cues within the actuator linear displacement range. Such algorithms will be explained later in this chapter as will the solution selected for the implementation in the Simulator.

\subsection{Washout Algorithm}

\subsubsection{General Description}

As previously explained, the main limitation of a Stewart platform used for motion cue reproduction, is the limited capability of linear displacement of the actuators. The washout algorithm is a Motion Cue Algorithm (MCA) that overcomes this problem by treating the angular velocity and linear acceleration, which have to be reproduced, to stay within the movement range of the platform. The washout algorithm consists of a combination of scaling schemes, filtering and a tilt coordination (used to generate linear acceleration cues using platform tilt and gravity). The implementation chosen for this thesis was the one described by Reid et al. in their Flight simulation motionbase drive algorithm reports $([150],[151],[152])$. The final algorithm was implemented using a similar scheme to the one presented in the second version of the washout algorithms described in [151]. Such implementation was modified to take into account the platform cues calculation (leg extension and acceleration) based on [40], instead of using the approximations (leg velocity and acceleration) chosen by Reid et al. in their work. The three algorithms described in [150], [151] and [152] are the Classical Washout, the Optimal Washout and the Adaptive Washout.

\subsubsection{Classical Washout}

The Classical Washout implementation developed by Reid et al. is based on the work presented in [7], [127] and [28]. The Algorithm inputs are related to a specific point of reference in the cockpit that will be related to a point in the Simulator frame of reference. As explained in section 5.3 of [150], for a better low-frequency acceleration reproduction (tilt-coordination) the Simulator reference point has to be coincident with the centroid of the Simulator upper platform (where the motion cue algorithm will be calculated) reducing the associated actuators excursion. The inputs will be of two different types:

- The cockpit specific forces (Linear accelerations without the gravity).

- The vehicle angular rates (Angular velocities). 
As explained in [150] the Algorithm is separated into two motions channels:

- Translational motion.

- Rotational motion.

The Translational Motion Channel: In this channel, the specific forces of the vehicle are passed through a scaling and limiting process acting as a non-linear attenuator. Then the gravity force is added and the intermediate acceleration variable is passed through two high-pass filters. The first filter aims to eliminate the lowfrequency acceleration content (that cannot be reproduced by the motion platform). The acceleration signal is transformed from the vehicle frame of reference to the inertial frame of reference (Earth-fixed, could be placed into the motion platform base but has to be chosen depending on the problem to solve) because it presents fewer disadvantages than filtering in the simulator frame of reference (as explained in section 5.6 .3 of [150], "if the washout process is carried out in Fs it can result in undesirable offsets in the motion-base hydraulic actuators", where Fs is the frame of reference attached to the simulator cab or upper platform). After that, the second high-pass filter is applied to restrict the movement of the platform actuators (as explained in section 5.1 of [150], "by high-pass filtering a2, which represents acceleration components in $\mathrm{Fi}$, it is possible to ensure adequate motion travel restriction at the expense of introducing some degree of cross-coupling of sensed motion stimuli back in Fs", $\mathrm{Fi}$ is the above-mentioned Earth-fixed reference frame). The resulting acceleration is fed into the motion cue calculation algorithm.

The Rotational Motion Channel: The rotational channel generates the platform Euler angles which are separated into two components. The first component is obtained after treating the vehicle angular rates using a similar process to the one qualitatively described in the translational channel (Scaling and limiting the signal, then using the first high-pass filter, transforming the signal to the platform frame of reference and using the second high-pass filter). The second component is obtained from the tilt coordination algorithm. The tilt coordination algorithm simulates translational inertial acceleration that cannot be generated using the translational motion of the platform. Instead, it is simulated using the platform tilt and gravity force. The method is intended to simulate the low-frequency part initially filtered from the vehicle specific forces. Such specific forces are passed through a low-pass filter, and the resultant translational motion needed to reproduce such forces is translated in 
its corresponding platform tilt (Using gravity and trigonometric expressions). The magnitude of these tilt-translated specific forces cannot be fully simulated but it is possible to reproduce the direction of application. The angular motion needed in the tilt coordination has to be kept below the pilot angular motion detection threshold using a rate limiter. The specific Euler angles from the tilt coordination are added to the Euler angles derived from the vehicle angular rates. Then they are fed into the motion cue calculation algorithm.

The motion algorithm is presented in Figure 11.1 of [151] and the original flowchart of the implementation is presented in Figure 4.7. A deeper explanation of this specific implementation of the Classical Washout algorithm is presented in chapter 5 of [150], in chapter 11 of [151], [152] and completed in [120].

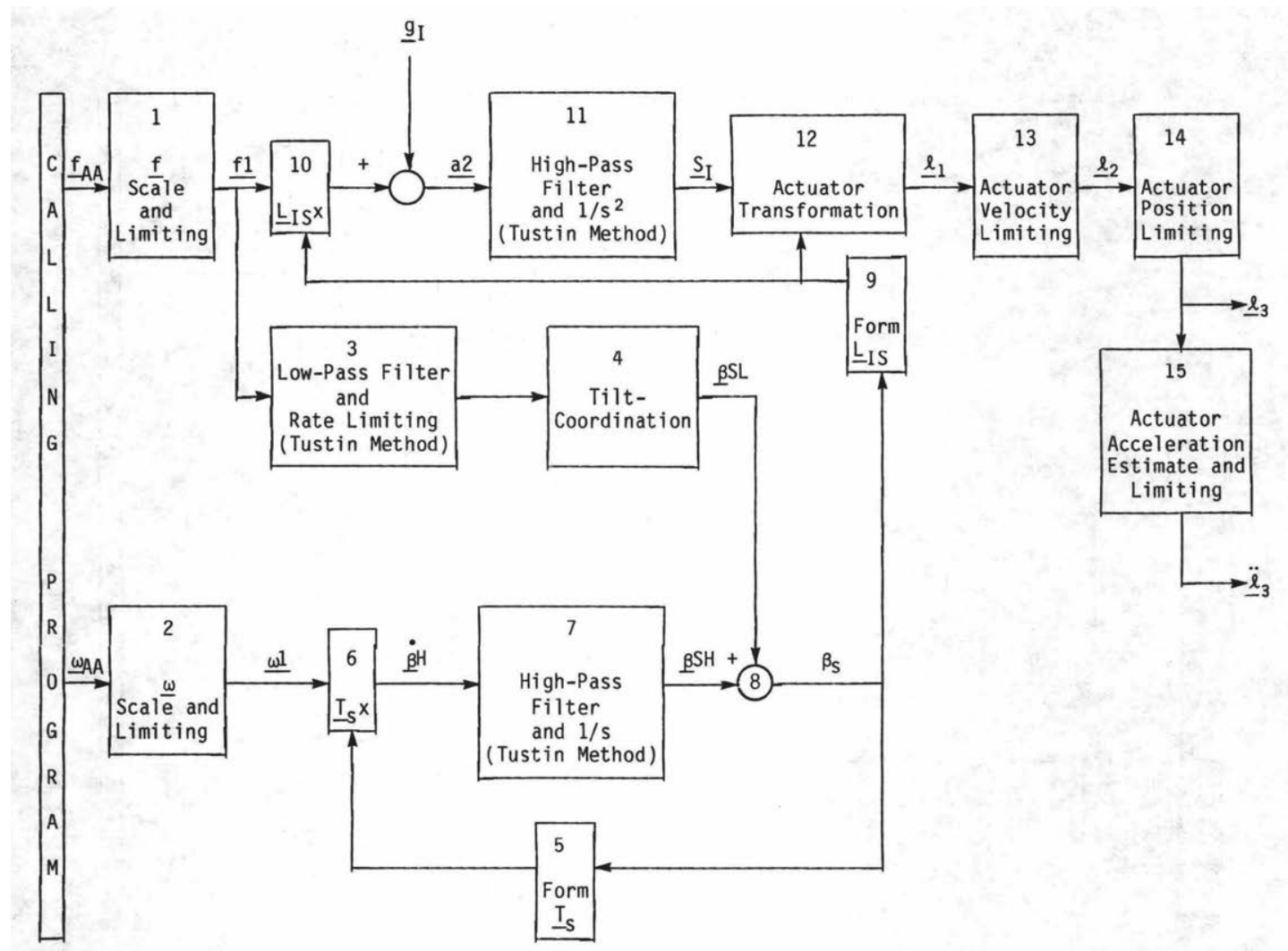

Figure 4.7: Original flowchart of the Classical Washout implementation. Source: Reid et al., Figure 14.1 in [151]

Table B.4 in Appendix B shows the kinematic cues (leg extensions and accelerations) for the Stewart Platform, calculated using the Classical Washout and the platform kinematic algorithms, of the first four seconds of the simulated Moth transition 
from non-foiling to foiling (described in section 3.7.3). Table 4.1 shows the cumulated linear sensed error between the onboard sensed accelerations and the motion platform generated sensed accelerations for the complete simulated transition, showing similar overall performance than the Adaptive algorithm (please refer to Table 4.2). The tuning parameters used are the ones proposed by Reid et al. in [151]. These parameters have not been optimized for this type of simulations (large cumulated error in X-Axis and unwanted initial oscillations in Z-Axis) and new parameters should be used. The platform geometry used is similar to the geometry of the SIROCCO hexapod from Symetrie [168].

Table 4.1: Cumulated linear sensed error for the Classical Washout

\begin{tabular}{|c|c|c|c|c|c|}
\hline Error X & Error Y & Error Z & Error X Rot. & Error Y Rot. & Error Z Rot. \\
\hline 18.624 & 0.872 & 6.590 & 0.041 & 0.825 & 0.001 \\
\hline
\end{tabular}

\subsubsection{Optimal Washout}

The Optimal washout implementation developed by Reid et al. is based on the work presented in [160] and [80]. The input for this algorithm is similar to the input in the Classical algorithm, but the vehicle specific forces are replaced with the vehicle linear accelerations. Inside the algorithm, the input is transformed and expressed in a specific frame of reference located in the pilot's head. The algorithm is based on a combination of an optimal filter coefficient design tool and the washout algorithm itself (implementing such optimal filters). The design tool solves the linear quadratic optimization control problem to obtain the optimal filters (form, order and characteristics) relying on the human vestibular response (the whole procedure is explained in chapter 6 of [150]) to minimize the perception error. Once the filters are obtained, the transformed input (expressed in the pilot's head frame) is treated. Following the same format as the Classical algorithm, it is separated into two motion channels:

The Translational Motion Channel: The accelerations are scaled and limited and translated to the inertial frame of reference (Earth-fixed, could be placed into the motion platform base, but has to be chosen depending on the problem to solve) to avoid sub-optimal control and Simulator displacement offsets. Then they are passed to the first high-pass filtering stage. This stage has a specific high-pass filter for each of the components of the accelerations $(\mathrm{X}, \mathrm{Y}, \mathrm{Z})$. The resulting signal is passed to 
a second high-pass filter (which is the same for all the components). The result is passed to the motion cue calculation algorithm.

The Rotational Motion Channel: The rotational channel is also divided into two components. The first component is derived from the transformed angular rates. Such angular rates are first scaled and the yaw component is then filtered using a high-pass filter. The second component is formed by the Euler angles related to the tilt coordination. Here the scaled and limited X-component and Y-component of the already scaled and limited acceleration are individually filtered using a specific lowpass filter for each one. Then, the tilt angles are determined by the tilt coordination. The two components are summed and expressed in the platform Euler angles. Finally, they are fed into the motion cue calculation algorithm.

The Optimal Washout coefficient design algorithm (as explained by Nahon et al. in [120]) rely on the features of the modelled human motion-sensing apparatus and user position. In fact, inside the washout algorithm the inputs are translated to the pilot's head frame of reference. This is why this washout algorithm will be more suitable to situations where the Simulator user has a clear and fixed handling position (for example: sailing in relatively big boats without changing the tack). The other algorithms present a more general approach and should be used when a certain user movement on the platform is expected, or in a multiple-user situation when the simulator is used at the same time by the helmsman and the trimmer, for example.

The motion algorithm is presented in Figure 12.1 of [151] and the original flowchart of the implementation is presented in Figure 4.8. A deeper explanation of this specific implementation of the Optimal Washout algorithm is presented in chapter 6 of [150], chapter 12 of [151], [152] and completed in [120]. 


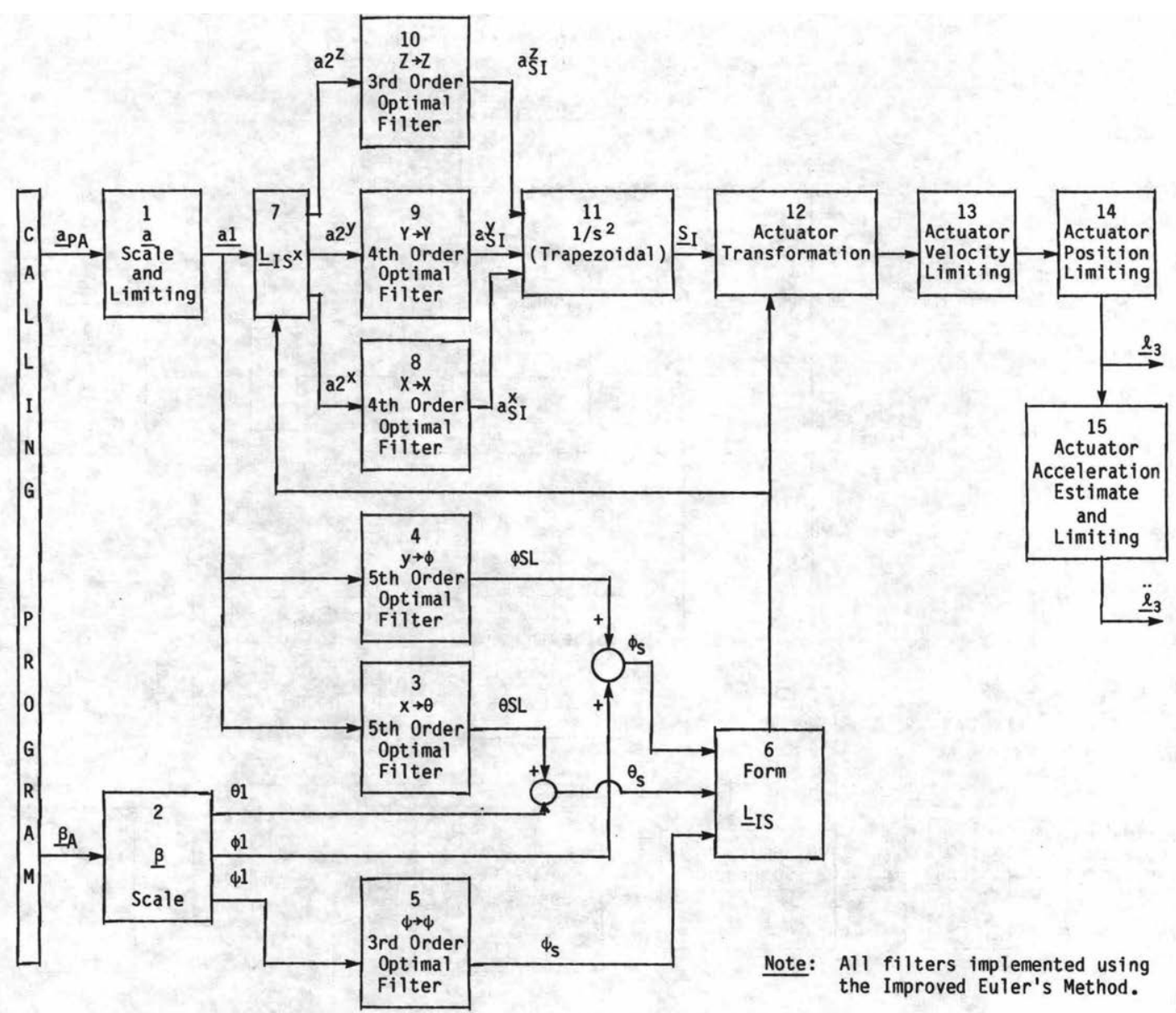

Figure 4.8: Original flowchart of the Optimal Washout implementation. Source: Reid et al., Figure 14.2 in [151]

\subsubsection{Adaptive Washout}

The Adaptive Washout implementation developed by Reid et al. is based on the work presented in [126], [128] and [1]. The algorithm is based on a combination of adaptive filters. Some of the filter's coefficients are changed in each time step in order to minimize a specific cost function. The inputs of the algorithm are the same inputs as the Classical, but the filtering scheme is different. For instance, the specific forces and angular rates are scaled and limited (and the acceleration is added to the specific forces) and then transformed into the inertial frame (the same frame used in the Optimal algorithm). A set of adaptive gain filters is then used separately for heave (Z linear Acceleration) and yaw (angular rate) channels and combined (to obtain a tilt coordination effect) for Surge-Pitch (X linear Acceleration with pitch angular rate) and Sway-Roll (Y linear Acceleration with roll angular rate). The result is fed into the motion cue calculation algorithm. Nahon et al. explain in [120] that this 
algorithm gave the best results based on user evaluations and is the method with a higher computational cost. The Classical algorithm presents a less refined approach and is computationally less expensive.

The motion algorithm is presented in Figure 13.1 of [151] and the original flowchart of the implementation is presented in Figure 4.9. A deeper explanation of the specific implementation of this algorithm is presented in chapter 7 of [150], chapter 13 of [151], [152] and completed in [120].

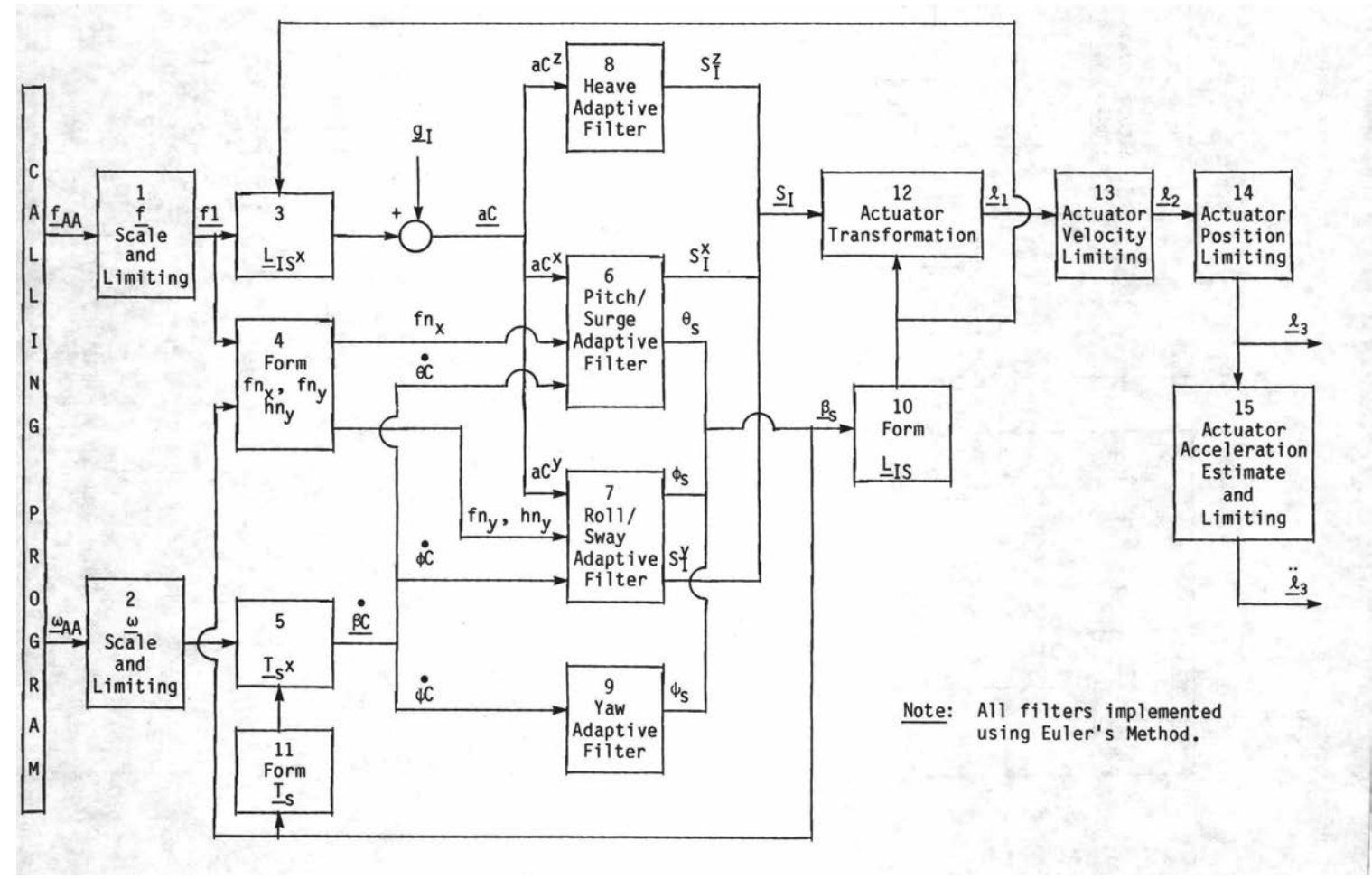

Figure 4.9: Original flowchart of the Adaptive Washout implementation. Source: Reid et al., Figure 14.3 in [151]

Table B.5 in Appendix B shows the kinematic cues (leg extensions and accelerations) for the Stewart Platform, calculated using the Adaptive Washout and the platform kinematic algorithms, of the first four seconds of the simulated Moth transition from non-foiling to foiling (described in section 3.7.3). Table 4.2 shows the cumulated linear sensed error between the onboard sensed accelerations and the motion platform generated sensed accelerations for the complete simulated transition, showing similar overall performance than the Classical algorithm (please refer to Table 4.1). The tuning parameters used are the ones proposed by Reid et al. in [151]. These parameters have not been optimized for this type of simulations (large cumulated error in X-Axis and unwanted initial oscillations in Z-Axis) and new parameters should be used. The 
platform geometry used is similar to the geometry of the SIROCCO hexapod from Symetrie [168].

Table 4.2: Cumulated linear sensed error for the Adaptive Washout

\begin{tabular}{|c|c|c|c|c|c|}
\hline Error X & Error Y & Error Z & Error X Rot. & Error Y Rot. & Error Z Rot. \\
\hline 18.646 & 0.854 & 13.165 & 0.015 & 0.811 & 0.001 \\
\hline
\end{tabular}

\subsubsection{Notes on the Implementation}

In the last years, new approaches have appeared. One of the most promising is the Washout Algorithm based on Model Predictive Control presented by Dagdelen et al. in [38] for automotive driving simulators. The idea behind the algorithm is to reproduce the vehicle motion as long as possible before reaching the limits of the platform which is then washed out to the initial position. In each iteration of the time-domain simulation, the algorithm will try to predict the following platform states, minimizing the squared perception error, while trying to remain within the platform range using a predictive control sequence (starting to wash out the platform below the user perception threshold after two predicted iterations). A predictive convex optimization problem is solved for each iteration, becoming computationally expensive (an approach using pre-calculated states before the time-domain simulation with a single-step optimization process is proposed in [38] to minimize the computational effort). However, this method is very easy to tune. This algorithm has not been implemented based on the suggestion made by Garrett et al. in [63] that "the resultant motion would feel unusual to the driver" in comparison to the more traditional algorithms previously described. Such algorithms were used and tested over the years in different simulators and could be considered a more robust approach.

Similar work has also been carried out for other vehicles (with some examples) such as:

- A motorcycle in [9].

- A helicopter in [159]

- A car in [27]

- A truck in [176] 
The recommended washout algorithm for the sailing boat simulator has to present higher flexibility and better results. Even if the Adaptive Algorithm is more computationally expensive than the others (for each time step it changes some of the filter coefficients in order to minimize a specific cost function), it is the most flexible along with the Classical one (they use a platform-based frame of reference instead of a frame of reference based on the user head position like the Optimal one) and presents the best results in comparison to the two other algorithms, as explained in [120].

\subsubsection{Washout Algorithm Tuning}

One of the main difficulties while dealing with washout algorithms is the selection of a proper set of filter coefficients and parameters. This is one of the most important tasks as choosing the wrong ones could lead to a poor motion reproduction and the generation of false motion cues. In some cases, the coefficients could be determined using special procedures (like the optimal washout algorithm), but even in these cases there are some parameters that have to be chosen. Normally, the parameter and coefficient selection is based on three elements:

- Motion worst-case scenario.

- Tuning engineer experience.

- Simulator user feedback.

In order to make this task easier, a different approach could be proposed: instead of using the three elements listed before for the initial tuning the idea is to use genetic algorithms, the vestibular model and time history information of the motion of the boat to automatically determine the needed coefficients and parameters. All of the elements present in this approach will be described in the following sections.

\subsubsection{Vestibular Model}

The vestibular system, in combination with the vision system, represents the major components of motion recognition of the human body (other human systems are also responsible for motion detection but they are normally related to relative body motion or are of less importance). The peripheral vestibular apparatus is in charge of sensing linear acceleration and rotation velocity of the human head. 
As explained by Khan et al. in their review [92], the linear acceleration, gravitational forces and head tilting are sensed by the utricle and saccule, both located in the static labyrinth in the human inner ear. The utricle is oriented to sense in the horizontal plane and the saccule is oriented to sense in the vertical plane. They contain a sensory structure called macula covered with a gelatinous membrane with calcium carbonate particles called otoliths. When the head is moving, shear forces appear between the macula surface and the aforementioned membrane. Such forces bend the macula hair cells allowing acceleration sensing. Rotation velocity is sensed by three semi-circular ducts, located in the bony semi-circular canals in the inner ear. The superior, posterior and lateral semi-circular ducts are oriented to allow the sensing of angular acceleration and head rotation in the three spatial dimensions. They are filled with a fluid called endolymph and both ends of the duct are connected to an enlarged cavity called the ampulla. The sensory structure (crista ampullaris) is located inside the ampulla which is covered by a gelatinous matter called cupula. When the head experiences a rotational acceleration, the endolymph flows through the duct deflecting the cupula in the opposite direction to the movement and bending the hair cells located of the crista ampullaris. In the case of a rotational deceleration, the flow will force the cupula to deflect in the same direction as the movement.

All of the previously explained systems behaviour can be modelled using mathematical expressions to be able to obtain the sensory response of the vestibular system when the human is subjected to a specific motion. Even if the models do not take into account all the sensory responses of the vestibular system (or other motion sensors of the body) they can be considered a good approximation of the primary sensations (as explained in chapter 3 of Reid et al. [150]). The implementation used in the tuning algorithm is based in the one presented by Reid et al. in [151] which in turn is based in [70], [191], [135] and [112]. The dynamics of each sensory system are modelled using differential equations solved with a modern object-oriented implementation of Gear's method for stiff differential equations. With that in mind both sensory subsystems are modelled using:

- An overdamped torsional pendulum combined with a first order high pass filter for the cupula deflection in the semi-circular ducts.

- The model proposed by Meiry and Young ([191], [112]) for horizontal motion of seated and supine subjects applied to the three axes of motion as explained by Reid et al. in [150]. 
One of the main advantages of the motion reproduction perspective is the fact that the vestibular system generates responses over specific thresholds. They are included in the qualitatively described vestibular models. Following Reid et al. indications in section 3.3 of [150], the selection of such thresholds follows a conservative approach. Lower values measured in the best conditions for detection process (using subjects in isolation) are used instead of the thresholds values measured in normal conditions. This allows generation of a more demanding filter tuning and the consequently motion reproduction. A more conservative approach with even lower values (taken from modern sources) are the ones presented in the vestibular section of Thöndel's paper [176]. They could be used for even more demanding tuning.

\subsubsection{Genetic Algorithms}

As previously explained, genetic algorithms can be used to tune the filter coefficients. This approach is presented by Thöndel in [176] where a genetic algorithm is used to tune the washout parameters, minimizing a fitness function. This function takes into account the sensed quadratic error, the platform jerks (the rate of change of acceleration which humans are sensitive to) and a specific assessment of physiological qualities of the washout filter. The approach proposed here is to individually minimize each of the degrees of freedom sensed quadratic errors (which is the quadratic difference between the vestibular response of the boat acceleration and the platform acceleration) and jerks, using the platform motion and acceleration limits as constraints. Multi-objective algorithms are not suited for optimization problems with more than 3 objectives, this is the reason why many-objective optimization has to be used. The problem could be set by combining the sensed quadratic error and the jerks for each DOF (resulting in minimizing 6 objectives) or individually minimizing all the 12 elements (12 objectives optimization problem).

Three algorithms are then selected:

- The most used multi-objective algorithm NSGA-II, presented by Deb et al. in [44], as the testing base (knowing that is not suitable for many-objective problems).

- A parallelised adaptation of the constrained MOEA/D algorithm based on Asafuddoula et al. paper [2] and Nebro et al. paper [122].

- An adapted version of the NSGA-III presented by Deb et al. in [43] and by Jain et al. [84], using the recommendations proposed in the MOEA framework 
for this specific algorithm (since the actual code is not released to the public). Some of the tests carried out by Deb et al in [43] were replicated with this adapted version showing good correlation (same order of magnitude) for the inverse generational distance metric.

To test which algorithm is the most convenient, a simpler version of the tuning algorithm was used using only linear sensed error and a specific platform motion geometry and characteristics (a commercial Stewart platform was modelled on this occasion). The time history information used for these tests is an on-board recorded sailing data of a Moth foiler regatta sailed by Benoit Marie of France, and kindly provided by him. The first tuning algorithm consisted of a 6 objectives constrained optimization problem. The tabular results are presented in Appendix E.

As expected, both MOEA/D and NSGA-III presented better results than NSGAII since they are meant to be used in many-objective optimization problems instead of multi-objective ones (as the NSGA-II is).

The best performing algorithm was the parallelised constrained MOEA/D version. It presents two main disadvantages: it relies heavily on external weighting vectors to reach a proper solution for a specific number of objectives (as explained by Ishibuchi et al. in [81]), and it needs a large population size for better results.

However, NSGA-III presents similar results with two main advantages: it does not need specific weighting vectors and needs a much smaller population size for similar performance. It is therefore the algorithm chosen in this thesis for the tuning process as it presents less computational effort and more flexibility to set the optimization problem.

\subsubsection{Time History Information Generation for Coefficients Training}

To generate the linear acceleration and rotation velocity time history information needed by the tuning algorithm three methods can be used:

- The easiest source of information is to simulate a controlled run or regatta under certain wind and sea conditions using the Simulator (without the motion platform if pre-existing coefficient data is not available) similar to the timedomain simulated runs for the AC72 and the Moth Foiler presented in Appendix $\mathrm{A}$ and $\mathrm{B}$. 
- If the boat is already built and measured on-board training data is available for different conditions, such data could be used to generate the time history needed.

- If the boat dynamics are well known and the simulator will be used just for training and not for boat designing, then recorded or generated data could be examined to look for the worst-case scenario regarding all the 6-DOF.

All three options present their own advantages and disadvantages and need to be utilized in specific situations.

Regarding the data generated using a controlled run or regatta with the simulator, the main advantage is that all the conditions are controlled and all the information needed is generated easily without needing a constructed boat. If a Race Modelling Program is used or two boats are controlled and simulated in a one-on-one regatta, actual regatta conditions could be reproduced and the information obtained will be more accurate. The main disadvantage is that the information generated relies on forces and moments models. If the models are not validated or the level of reality needed by the crew is not reached, then the motion simulation and the overall Simulator experience will be far from ideal.

Regarding measured on-board acquired data, it potentially presents the highest level of accuracy and realism because the actual phenomena are being measured (knowing that on-board data can be sparse and can present unknown errors derived from measurement drift, wrong calibration, human trim, wind shear. etc.). The main disadvantages are that you need the actual boat to perform the acquisition, the weather conditions cannot be controlled and large on-board acquisition campaigns are very time-consuming and economically expensive when trying to cover the full spectrum of wind and sea conditions. When simulating and designing new configurations, normally the boat is still being built; or, if it is built, the configuration analysed is not, making this procedure useless.

Finally, if enough measured on-board sailing data is available or enough data has been generated using numerical tools and the main goal of the Simulator is training (no further design involved), then a conventional approach of worst-case scenario could be used (after carefully analysing all the data). 


\subsubsection{Filtering Parameter Selection Using a Vestibular Model and Ge- netic Algorithms Training}

The tuning algorithm presents the same approach with slight variations for each of the washout algorithms studied. The methodology implemented is the following:

- The genetic algorithm problem is set (all the optimizing parameters are defined) and the objectives (with 6 or 12 objective functions depending on if sensed quadratic error and jerks are taken into account separately for each DOF) and constraints (motion platform actuator excursion and acceleration) are defined. The optimization process begins:

- A parallelised approach is used and for each execution instance of the genetic algorithm an object containing the boat time history information is generated

- The time history acceleration and velocity information are then processed into two different paths:

* It is directly fed into the vestibular model to obtain the actual boat sensed motion.

* It is fed to the specific washout algorithm and then the platform linear acceleration and angular rates are passed to the vestibular model.

- The maximum sensed quadratic errors and jerks are calculated for each DOF and their value assigned to objective functions and checked by the genetic algorithm.

- The platform maximum and minimum leg extension and acceleration are assigned to the constraints and checked by the genetic algorithm.

- This process is repeated for all the population members of each generation.

The main difference in this methodology for each specific washout algorithm is the parameters to be tuned. For the classical algorithm (qualitatively described in 4.4.1.1), all the parameters related to the filters and scaling stages are tuned. For the adaptive washout (qualitatively described in 4.4.1.3), only the initial filter values and algorithm parameters are tuned. For the optimal washout (qualitatively described in 4.4.1.2), the tuning is different because the filter parameters are calculated by the coefficient design algorithm (chapter 6 of [150]) and the rest of the parameters which are not calculated with the coefficient design algorithm are tuned with the presented 
method (this case could be considered a mixed tuning approach).

One of the key aspects of successful motion platform tuning is the way the boat time history information is generated. Normally, the worst-case scenarios are used for coefficients determination, but this could generate a poor use of the platform ranges of motion since in normal operation such scenarios are not always reached. To create the data different approaches are proposed:

1. To generate data for different wind and sea range windows and calculate in different tuning sessions the washout coefficients for each window (low wind conditions, medium wind conditions, high wind conditions, etc.). Tabular coefficient data is obtained and the best parameters are chosen for each of the simulated conditions. These parameters will be well suited to the usual accelerations and angular rates boat range for each specific condition. This is the reason why, within that range, it maximizes the platform motion range usage, but could lead to higher number of false cues when larger accelerations and angular rates than usual are generated during the simulation.

2. To generate data using all the expected wind conditions that the boat will face (from lower to higher winds) in only one long time-history dataset. It allows possible wind shifts and changes during the regatta to be taken into account. This method could be considered as an intermediate approach between the specific range window and the worst-case scenarios. It presents the best trade-off between platform motion range usage and false cue generation. It is the suitable method when a new design is being developed and the worst-case scenario is unknown.

3. To generate data using the worst-case scenarios for boat linear acceleration and angular rates. The parameters obtained using this method will be able to reproduce extreme situations within the motion range of the platform however usual accelerations will be reproduced using a reduced platform motion range. This is the reason why it presents a poorer platform motion range usage but also a lower false cue generation.

The main disadvantages of this tuning method are directly related to the vestibular model usage. It relies directly on the features of the modelled human motion-sensing apparatus and on the user head position. A trade-off has to be found if a moving user is expected inside the simulator (while changing tack or when the user position 
is not clearly fixed as in dinghy sailing). A multi-position training approach could be used by generating acceleration and velocity data in different cockpit positions and optimizing the washout algorithm parameters for the multiple positions at the same time in a combined time history.

For a wide approach, if the initial washout tuning process is made using the described technique, the recommendation is to use the adaptive washout algorithm with time history data containing information with all the expected wind conditions (approach 2 above) combined with the multiple-position approach. After the initial process, a manual fine-tuning process has to be made based on the Simulator user opinion.

Potentially, similar tuning results could be achieved using other tools. For example, replacing the genetic algorithms by neuronal networks. 


\section{Chapter 5}

\section{Visualisation Interface}

\subsection{Vision as the Main Source for Motion Simula- tion}

The human visual system is capable of recognizing motion patterns based on a succession of images received by the retina. This image pattern succession associated with object-motion or self-motion, known as the optic flow, was first presented by Gibson in [65]. Later studies, like the one carried out by Dichgans et al. in [46], showed that self-motion perception could be induced using large-field optic flow combined with vestibular stimulations.

The optic flow is directly related to the speed and direction of the subject motion but also to the depth distribution all along the perceived scene. As explained by Britten in [21], all the local velocity vectors of the different points in the perceived optic flow emanate from a single point called Focus Of Expansion (FOE) following a radial pattern and matching the actual motion direction (heading). This property allows the subject to determine the motion heading. Motion velocity is determined by the perceived velocity of the optic flow. Finally, distance can also be determined because of motion parallax. The local points velocity will depend on the distance to the subject - the bigger the distance, the slower they will move. For linear self-motion patterns, the optic flow can be enough for heading, velocity and distance perception. But if gaze-changing is involved (via a combination of eyes, head or torso movements) when the subject is following a different point to the FOE in the image, the optic flow is distorted producing a misalignment between the FOE and the motion heading (a phenomenon known as rotation problem). In this case, more information is needed to be able to compute the actual motion heading. Gaze-induced motions produce differ- 
ent motion patterns than linear motions. In the absence of self-motion, gaze-induced motions generate a uniform velocity pattern all across the optic flow which is depthinvariant. In opposition, the translation self-motion is associated with an expansive pattern which is depth-dependant. Theoretically, two different approaches were proposed. Some authors modelled the perceptual mechanism to identify gaze movements only relying on the visual information and the depth dependence (or independence) of the translation (rotation) component (like the model proposed by Perrone et al. in [132] and [133] or the one proposed by Royden in [154]). In such models, the subject will be able to identify the optic flow patterns and decompose the velocity vectors in two components when depth is present in the observed scene - the rotation component and the translation component. Other authors modelled such a mechanism with a combination of visual stimuli information and other extraretinal signals (like the ones presented by Ben Hamed et al. in [11] or by Hanada in [71]). Experimental studies show that the second approach seems more accurate. In Royden et al. paper [155] they noted that under many viewing conditions, when depth information is degraded or in high-speed eye movements situations, the subjects determined the motion heading more accurately than a simulated eye, determining that extra-retinal signals were used in such situations.

Regarding object-motion, the problem is even more complex. Object-motion adds a new layer of complexity to the already existing gaze-moving problem in self-motion (usually induced by the subject fixing the attention onto the moving object instead of the FOE). The optic flow will be formed by combined velocity information of selfmotion and object-motion relative to the subject. The brain also has to identify and separate the effects in the optic flow of the object-moving source (source separation problem). Two different approaches are presented regarding the way the brain solves the problem (like when dealing with rotation problem). Some studies propose that it can be solved by the means of the optic flow visual analysis (like the ones carried out by Matsumiya et al. in [104] or by Warren et al. in [185] and [186]). Others propose that alternative non-visual stimuli are needed (Wexler in [189] or Wexler et al. in [190]) as sources of information to identify and separate object motion from self-motion (like the vestibular response used as an independent information source to identify consistencies in the optic flow compared to pure self-motion). But even if both propositions are viable, the real neural mechanism remains unknown. 


\subsection{The Virtual Reality Engine to Represent the Optic Flow}

As was the case with the motion platform, even if we ignore the real neural mechanism behind motion recognition, from the Simulator point of view the only tool needed is one capable of representing three-dimensional moving images (containing depth information) of the environment. Since the 1980's, the video-game industry has developed the leading tools and techniques to offer a realistic and immersive gaming experience representing rich, visual, three-dimensional virtual environments. In such environments, the user can move and interact with virtual objects as if in the real world. In order to improve the visual experience, first-person video-games were developed to generate moving images that emulate a real optic flow. The user has the opportunity to feel like he is moving around a virtual world using visual cues. The modern tools that allow generation of such three-dimensional first-person visual cues are known as game engines or virtual reality (VR) engines ${ }^{1}$. They offer a wide variety of functions to easily generate a realistic optic flow.

\subsubsection{Simulator Needs}

From the Simulator point of view, modern-age computer graphics and VR engines offer all the tools needed to realistically represent the virtual world. For that reason, they will not be discussed here. Instead, the challenge is to find which available VR engine will suit the sailing Simulator best, mostly because of its own peculiarities and the interfacing with the rest of the elements. That decision was made based on four different requirements:

- Water and waves generation capacity.

- External Dynamic Linked Library (DLL) calling for VPP interfacing.

- Virtual Reality systems integration.

- Integrated Development Environment (IDE) integration and available coding languages.

\footnotetext{
${ }^{1}$ In the simulation industry, these engines are used to generate the visual virtual world, not a complete gaming experience. This is the reason why virtual reality engines will be used instead of game engines in this thesis.
} 
The physics calculation capabilities included inside the VR engines were not taken into account due to the fact that the VPP is responsible for such calculations. It was decided that the calling procedure would be made from the VR engine to a compiled dynamic linked library containing the VPP. This allows implementing multiple boats calling multiple and independent DLL instances (the VPP was developed using Object Oriented Programming (OOP) and can be called in each instance as an independent object) making it simpler and more flexible. The VR engines considered during the selection process were four commercial solutions readily available on the market.

- Unreal 4.12

- Unity 5.3.2

- CryEngine 5.10

- Unigine 2.2.1

It is important to note that even if free options were taken into account, it was decided that the four requirements previously listed were more important than the actual economic cost.

Unreal 4.12: Based on the information provided by the documentation [48], Unreal engine is compatible with multiple platforms. It allows the user to develop the application in $\mathrm{C}++$ and to call external code (in this case the time-domain VPP that handles the physical calculation) using a DLL (written in $\mathrm{C}, \mathrm{C}++$, etc), and can handle Common Language Runtime (CLR) .Net managed and unmanaged code inside the solution. It does not present sea water and wave generation built in but it can be added using a side project called Ocean project (not fully automated and based on Gerstner waves). It also allows VR devices integration.

Unity 5.3.2: Based on the information provided by the documentation [171], Unity is a multi-platform engine that allows $\mathrm{C}++$ and $\mathrm{C} \#$ coding to develop the project. It allows calling external code if it is compatible with Mono (CLR managed code) using a C\# script inside the Unity project. Visual Studio IDE integration is available for the managed code $(\mathrm{C \#})$. It does not present complex water management and wave generation built in, which would have to be achieved with specific coding from the ground-up. It is compatible with VR devices. 
CryEngine 5.10: Based on the information provided by the documentation [66], Cry engine is also multi-platform and it allows the use of $\mathrm{C}++, \mathrm{C} \#$ and LUA coding languages for the project development. External code can be called as a DLL directly if written in $\mathrm{C}++$ or using Cry Mono when written in $\mathrm{C} \#$. The engine allows built in water handling but the waves generation tools support was abandoned in version 2 of this engine. It is also compatible with VR devices.

Unigine 2.2.1: The last of the four VR engines analysed, Unigine, as specified in the engine documentation [30] is a multi-platform VR engine compatible with $\mathrm{C}++$, $\mathrm{C} \#$ and Unigine Script coding languages. It allows external code calling of COM DLL or CLR managed or unmanaged code using its full compatibility with the Microsoft Visual Studio IDE. This IDE can be used as the main development environment greatly simplifying the task of calling VR-engine external code. Because it is more oriented to the simulator industry, it presents a very simple and automated interface for complex water handling and waves generation (using a Beaufort scale) with realistic results. Finally, it is compatible with different VR devices.

For the purpose of this thesis, the VR engine selected was Unigine because of its water and waves generation capabilities and ease of use, the multi-screen and Virtual Reality headset compatibility and simplicity regarding interfacing to the VPP (the possibility of using the same IDE for the VR engine and the VPP in a single project). In fact, Unigine develops a specific VR engine for the simulation industry called UnigineSim with simulator-related specific tools such as landscape generation supporting real geo-coordinates and 64-bit precision coordinates for large scale environments. For a general overview of the VR engine implementation within the simulator please refer to the diagram in Figure 2.1. For a specific overview of this module implementation please refer to the diagram in Figure 5.1. 


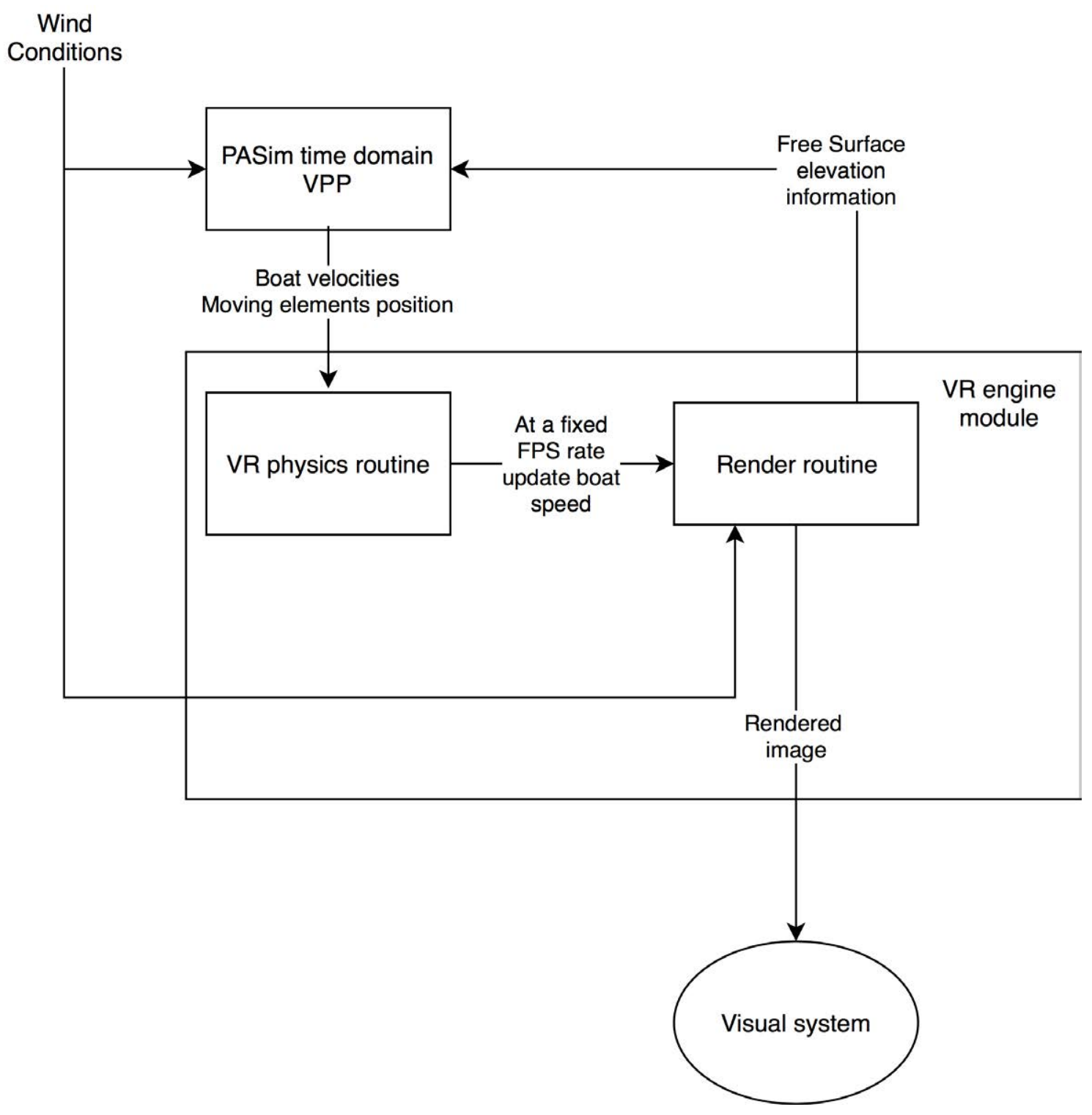

Figure 5.1: VR engine module block diagram

\subsubsection{A Tool to Generate Environmental Cues}

When a boat is sailing in the sea, it is under the influence of certain environmental conditions. The principal conditions that affect sailing are the true wind speed, true wind angle and the sea state as defined by wave size and steepness.

These environmental conditions present their own associated problems regarding their reproduction, but the real wind is the easiest part to handle. In fact, knowing only its angle and speed the time-domain VPP can perform all the apparent wind calculations affecting the sails and windage performance. There are no geometrical 
wind characteristics to represent visually other than how true wind speed and true wind angle affect the waves pattern (changing waves direction and associated with waves height). This is the reason why the wind information is mainly handled by the VPP. In fact, these two wind parameters could be handled in different ways:

- Reading a pre-set data file with true wind direction and speed linked to the coordinates where the boat is sailing in the virtual world.

- Both variables could be changed in real time by the engineer controlling the Simulator normally using slides that could be implemented inside or outside the VR engine interface.

Other information such as the wind speed and direction indications around the boat could be generated by the means of visual indicators within the VR engine (arrows or other) or by using voice indications to the Simulator user as if it were the on-board tactician.

As previously explained, one of the main requirements while choosing the VR engine was the ability to generate visually appealing and realistic water and waves. In this case, the geometrical representation will affect not only the visual aspect of the Simulator experience but also the boat behaviour. In fact, the waves influence on the boat could be modelled inside the VPP using the wave height information generated by the VR engine (along different boat sections modifying its hydrostatics and resistance). The actual wave behaviours can be modelled using complex mathematical fluid-dynamic models (for example the NHWAVE model developed by Derakhti et al. [45] based on RANSE). But, these approaches need a large amount of computational resources and they cannot be solved in execution time. They are more suitable for off-line simulations in specific or local studies than for execution-time wave state generation. Another problem associated with real sea states is that they are not regular and a certain amount of randomness is needed. Knowing all this information, different approaches could be used to address that task. Jerry Tessendorf in [172] presents the methodologies used for generating the geometric height map of the free surface that defines its represented final shape and all the visual techniques used to generate a reasonably realistic visual result (the optical process of reflection and refraction, colour behaviour and lighting effects). Regarding the geometrical height map generation and tesselation, different approaches could be used. The simpler one is to use a combination of sine functions to describe the height of the free surface as it is explained in Chapter 1 of [51] (In Isidoro et al. [82] this method is applied using 4 
sine and cosine waves). A more realistic approach consists of calculating the height map using a combination of Gerstner waves (also known as trochoidal waves). It was first applied in computer graphics by Fournier et al. in [60] and then modified by Peachey in [130]. The Gerstner waves describe the free-surface motion based on an exact solution of the Euler equations for surface gravity waves (for an incompressible fluid and infinite depth). Following this solution, each point of the free surface describes a circular motion as the waves pass by that can be calculated, obtaining the height map of the free surface that will be tessellated. The main advantage of such a technique is that it is very lightweight computationally. It is possible to improve the visual quality of the simpler methods while maintaining computational efficiency using the Vertex Texture Displacement methodology. It is presented in chapter 18 of [136] and it involves combining (usually no more than four) simpler harmonic or octave height maps tiled in space and time. To generate a more realistic representation of the waves field it is possible to use a complex approach based on statistical wave models and the Fourier transform. This statistical approach explained in section 4.3 of [172], expresses the wave height at a specific position in the free surface using a wave spectrum (analytical or semi-analytical) and a Fast Fourier Transform (FFT) based representation. In order to generate random wave height maps using this approach, Gaussian random numbers with spatial spectra of a prescribed form are used (section 4.4 of [172]). The main disadvantage of this method is that it requires more computational resources than Gerstner waves.

However, as explained by Tessendorf in [172], FFT method can be considered a "fairly realistic representation of typical waves on the ocean when the weather is not too stormy". In order to solve this limitation a specific procedure to generate choppy waves is introduced in section 4.6. The methods described earlier consider infinite or constant depth and flat bottom, but Tessendorf introduces in section 5.1 some modifications to take into account variable depth shallow waters.

The calculated water height map is then used to generate a grid of vertices (placing each of the vertices in the plane at the corresponding height information provided by the height map). Then triangles will be formed using those vertices, creating a surface mesh that will be rendered.

The visual aspects of the waves will not be discussed because they are usually implemented natively in the modern VR engines (at least in the selected one) and they do not affect the boat behaviour calculated by the VPP (some explanations about the different techniques used could be found in [172] and Chapter 2 of [51]). 
The main advantage of the selected VR engine is that the developer interface regarding the wave generation is simplified and related to a Beaufort scale (different sea states can be found in Figure 5.2). Each part of the scale could be associated with a range of real wind speeds, provided by the VPP or changed in real time by the Simulator engineer, and could be directly linked to the true wind direction (again provided by the VPP or changed in real time by the Simulator engineer) in order to change wave trajectories.

When implementing the VR engine, a number of free surface height probes were distributed in different places of the boat, obtaining the deformed free surface of the water in the boat geographic position. During a simulator iteration, after the VR engine has received and rendered the new boat position and attitude, the free surface height information from the probes is sent back to the time-domain VPP. This information affects the different force models during the following VPP calculation (in the case of the moth, hydrostatics of hull and foils, wand control and foil force models). 


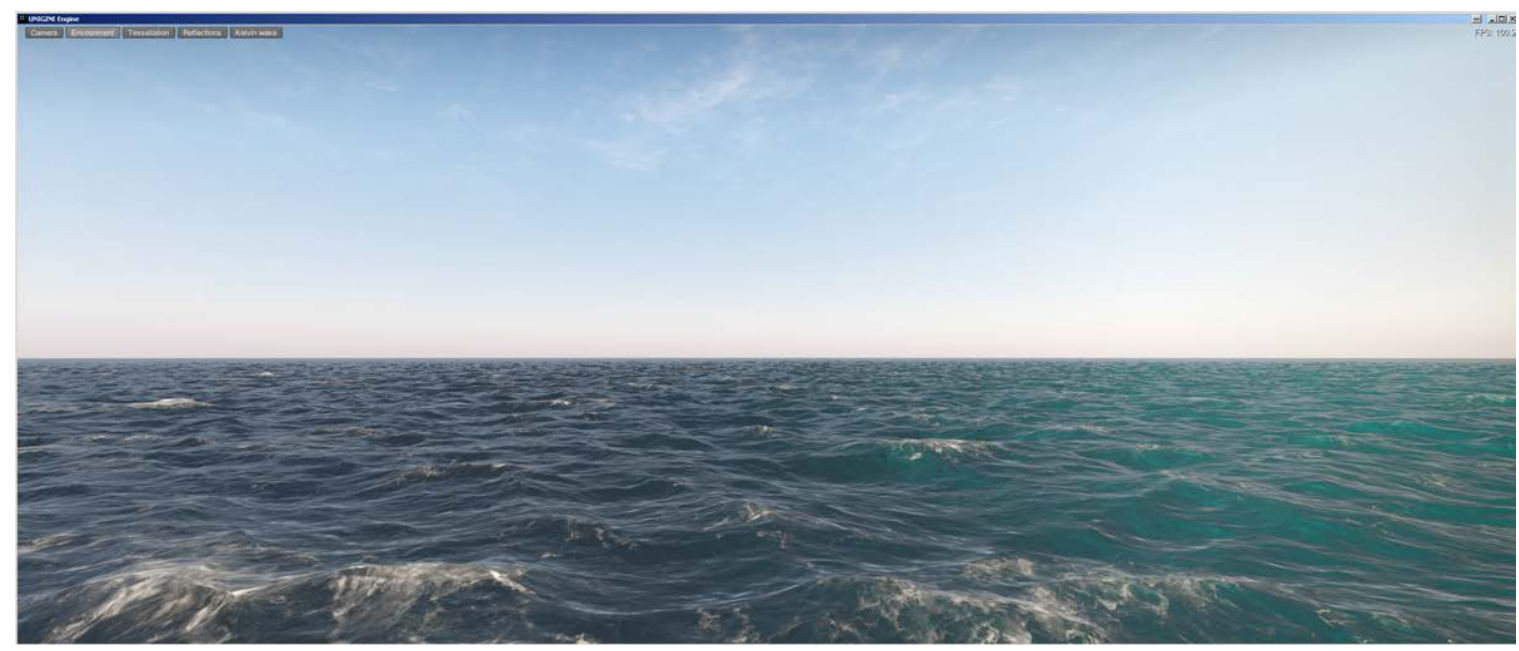

(a)

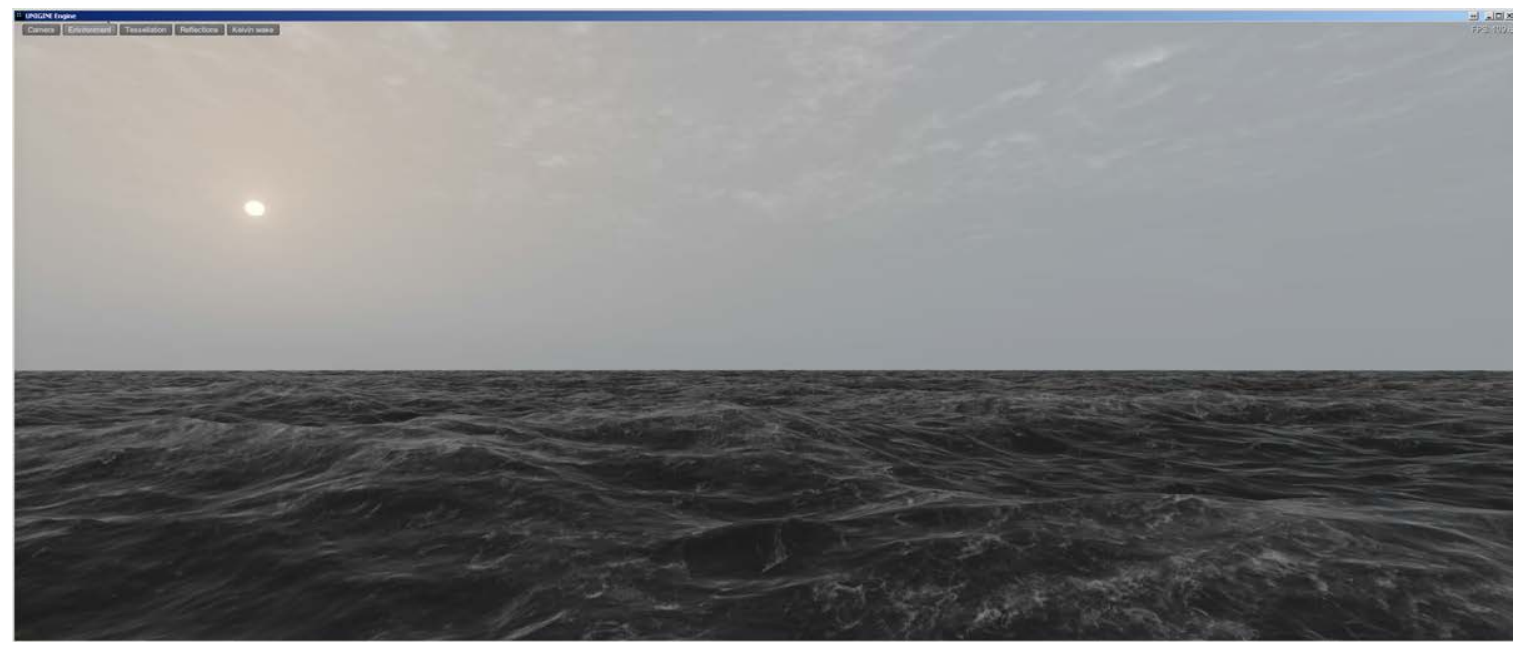

(b)

Figure 5.2: Beaufort 4 (a) and Beaufort 8 (b) sea states generated with Unigine

\subsubsection{A Tool to Show Vital Information}

When sailing, whether it be in highly advanced foiling boats, such as the AC50s used in the 2017 America's Cup, simpler ones, like a 10-meter sailing yacht cruiser, or even a dinghy, on-board electronics and displays provide essential sailing data (from basic AWS, AWA and BSP to a more complex set of data regarding navigation, timing information, boat attitude, course boundaries, etc). When using the simulator to improve design knowledge and training effectiveness, the same data (or more) available on-board has to be shown via the visualisation system (by means of the VR engine) using simple numerical indicators (like the one presented in figure 5.3) or more complex schemes and patterns. In fact, the platform can be used to plan and discuss 
how visual data is represented on-board. Building and testing display information systems prototypes is expensive and time-consuming because they involve hardware and software specific developments. The big advantage of the VR engine is that every crew or designer suggestion about on-board display position, the information available and how it is shown, can be easily modelled and assessed. There is no need for any physical modification of the instruments on board. In fact, a lot of work on how the information will be shown on-board could be made before construction of the cockpit has even started. Every hardware or software modification could be decided based on the Simulator run tests. Here the time and economic savings are double, there is no need to build any prototype or use the on-the-water training for such design tasks. It allows a faster development time of a very specific tool that has become more and more critical (one of the reasons for Emirates Team New Zealand's success in the $35^{t h}$ America's Cup was the way the crew interacted with visual information and changed settings on-board during the regattas).

As explained in Chapter 2 section 2.2.7, new VR engine technology and its compatibility with VR Headsets allows development of specific strategies for each of the crew regarding how and which information is shown using augmented reality. The VR Headsets allow specific reproduction of the field of view that each person will expect to have while sailing. The engineer in charge of the VR engine can work side by side with each crew member to design the best strategy for showing information. In fact, to be able to determine such strategy a huge amount of different possibilities have to be tested (taking into account all the sensory information that could be generated on-board and that will saturate crew perceptions). The VR engine will be the tool to specifically develop the most efficient and least disruptive visual strategy. It will also allow direct evaluation of the design process and how new display information strategies affect crew and boat performance. 


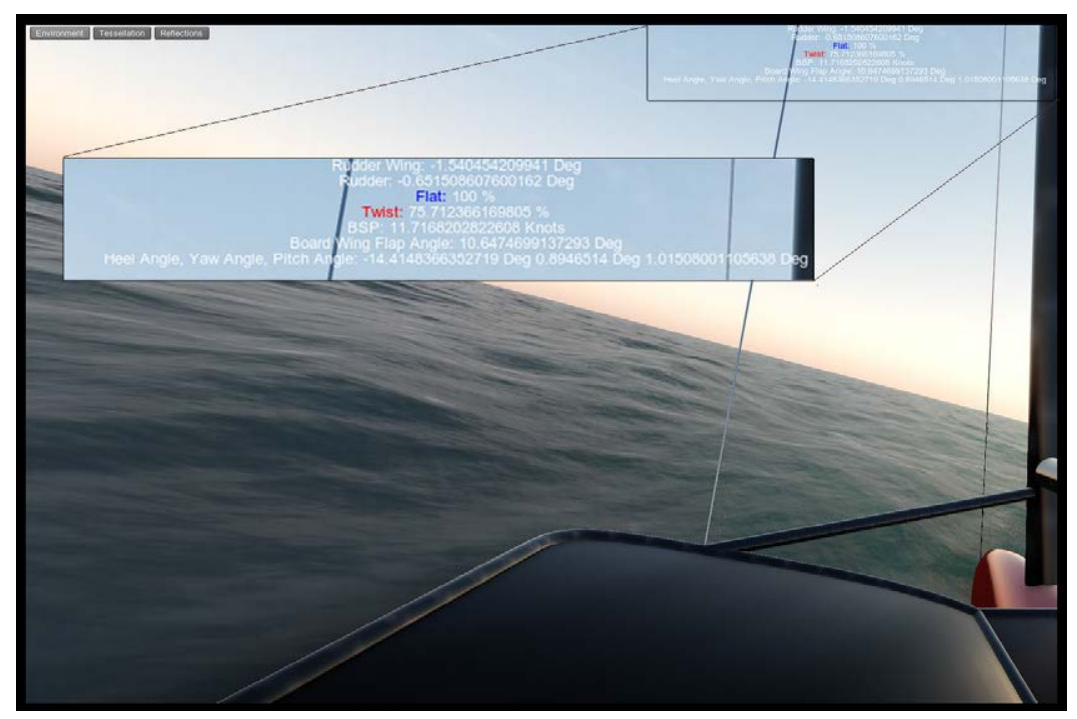

Figure 5.3: Basic way to show data for training and designing purposes using Unigine

\subsubsection{A Tool that Improves Two Boat Interaction Awareness}

The main sense used to assess two boat interaction is vision. In fact, while sailing with high Apparent Wind Speed, the noise, due to wind turbulence around the head, makes it difficult for the crew to sense the surroundings and the interaction between different boats using senses other than vision. The simulator could allow for early visual awareness training with how the crew is positioned in the boat and the overall boat dimensions and handling capabilities before the actual boat is built. Even if the VR engines sometimes offer a distorted sense of depth or one that is not truly realistic, visual training in the simulator will help the helmsman to improve judgement and on-board position as well as developing specific visual routines when approaching a mark or manoeuvring close to other competitors. Another interesting aspect of the visual system in a simulator is that visual ergonomic studies could be performed for each of the crew. Such studies allow for testing the actual cockpit position visibility and making changes on-board to improve comfort, visibility and situational awareness for each crew member. For example, checking tactician and helmsman visibility, or testing specific windage reduction strategies. 


\subsection{Specific Challenges of a Realistic Visual Expe- rience}

One of the main challenges when developing visual assets in sailing boat simulators is the need to reproduce non-rigid large elements like conventional sails. In other simulators, like the ones used in aviation the vehicles are usually represented by fixed or moving solid elements. Sail shape is something that will change over time because it is affected by the AWA, AWS and trim conditions.

Sail shape could be calculated using Fluid-Structure Interaction (FSI) codes. These types of codes need big amounts of computational resources and cannot be used in execution time. However, because it is only for generating visual cues, high accuracy is not needed and this method could be used to generate flying shapes for different AWA in successive simulations reproducing different conditions (Renzsch et al. in [153] presented the last version of their FLEXSAIL framework that allows obtaining downwind sails flying shapes using FSI) that later will be implemented within the VR engine

Another source of flying shape geometries is the visual geometric flying shape camera acquisition systems used with a scaled model of the boat inside a Twisted flow wind tunnel or with a real boat when sailing. These acquisition systems are based on different technologies such as:

- Photogrammetry, used by Mausolf et al. in [105] using a scaled model inside a wind tunnel and by Clauss et al. in [26] using a real boat (the TU Berlin's sail force dynamometer DYNA).

- Coordinate Measuring Machine (CMM), used by Ranzenbach et al. in [147].

- Time Of Flight (TOF), proposed by Fossati et al. in [57].

- Visual Sail Position and Rig Shape (V-SPARS), proposed by Le Pelley et al. in [96].

When generating the VPP forces and moments models (described in Appendix D) for the example boat, the Moth foiler, an experimental campaign was held in the Twisted Flow Wind Tunnel of the University of Auckland to obtain a complete aerodynamic model of the boat. During this campaign, the V-SPARS method was used to recover the flying shape of the Moth mainsail as can be seen in Figure 5.4. 


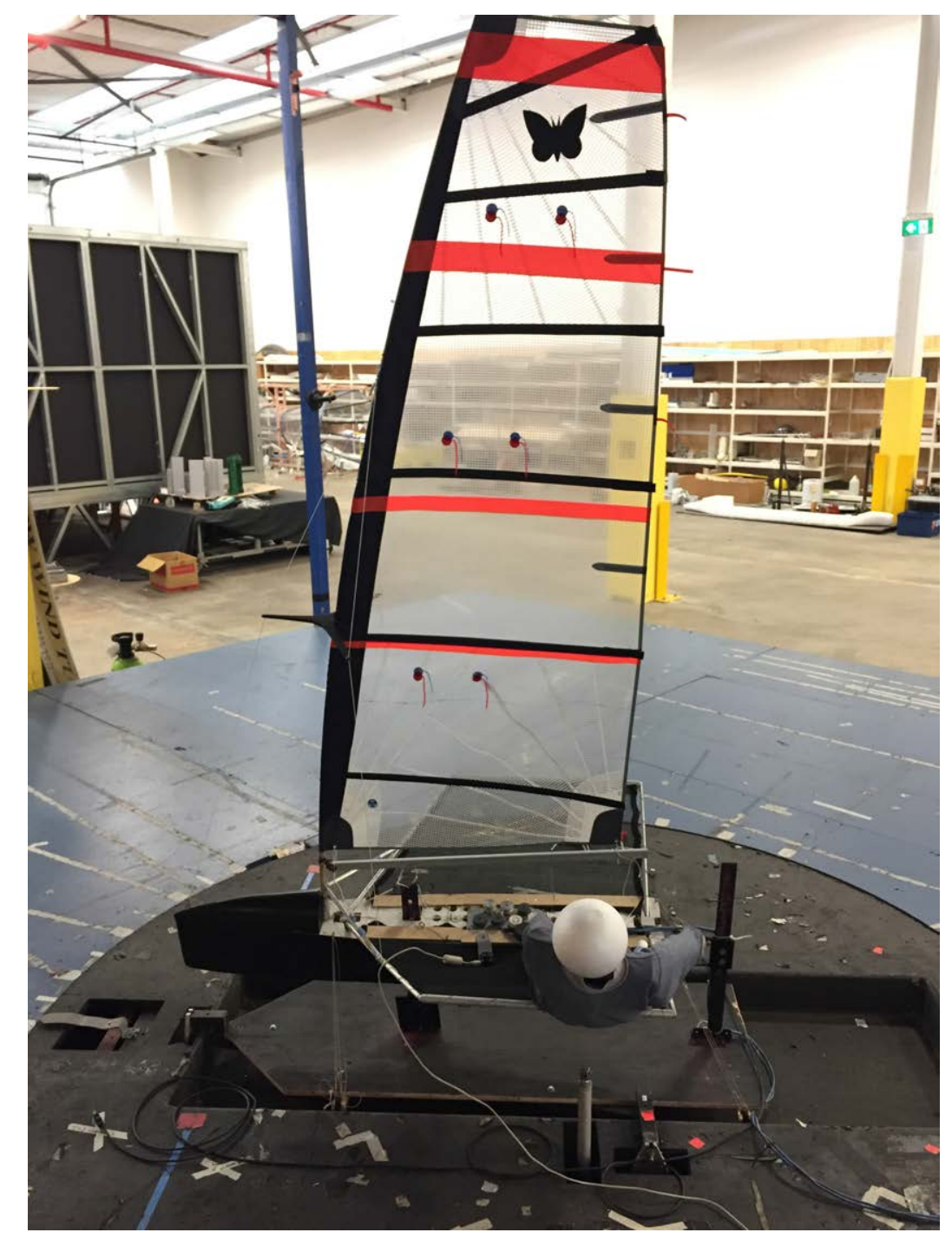

Figure 5.4: Moth foiler Scaled model with V-SPARs in the Twisted Flow Wind Tunnel of the University of Auckland

The V-SPARS campaign consisted of measuring the flying shape for a range of AWA in two different conditions: under straight flow and using the twisting vanes to generate wind twist. The idea behind this procedure was to verify if the assumption of straight flow testing was correct for high-speed boats. The main advantage of this system is its simplicity, with a limited number of cameras (one camera per sail and tack) and the use of reflective stripes, different slices and rig position could be determined with little pre-processing and post-processing work. Even if another method could be more accurate (part of the sail has to be "extrapolated" because only a limited number of sail slices are measured) for the purpose of visualisation and shape comparison it was considered more than enough. An example of the processed 
image and the preliminary three-dimensional result (with a partial reconstruction of the mainsail) for a twisted flow at 45 degrees of AWA is shown in figures 5.5.

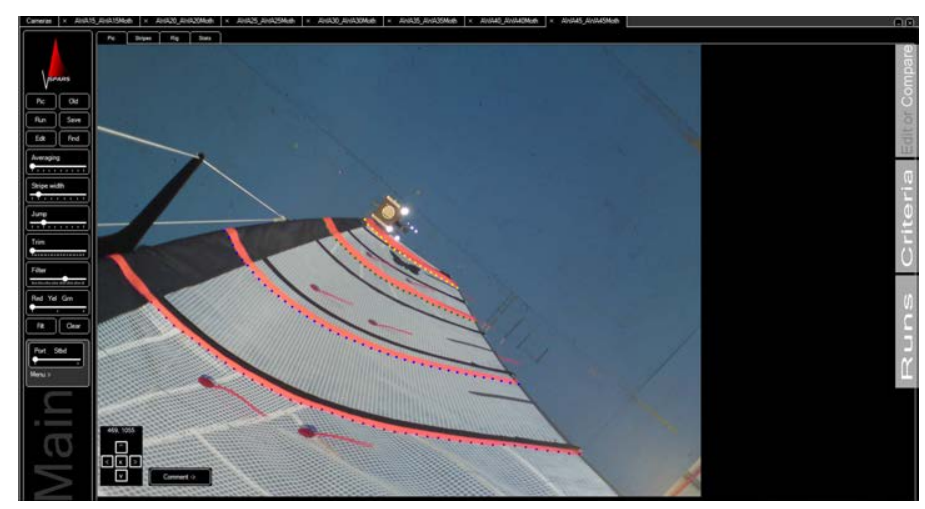

(a)

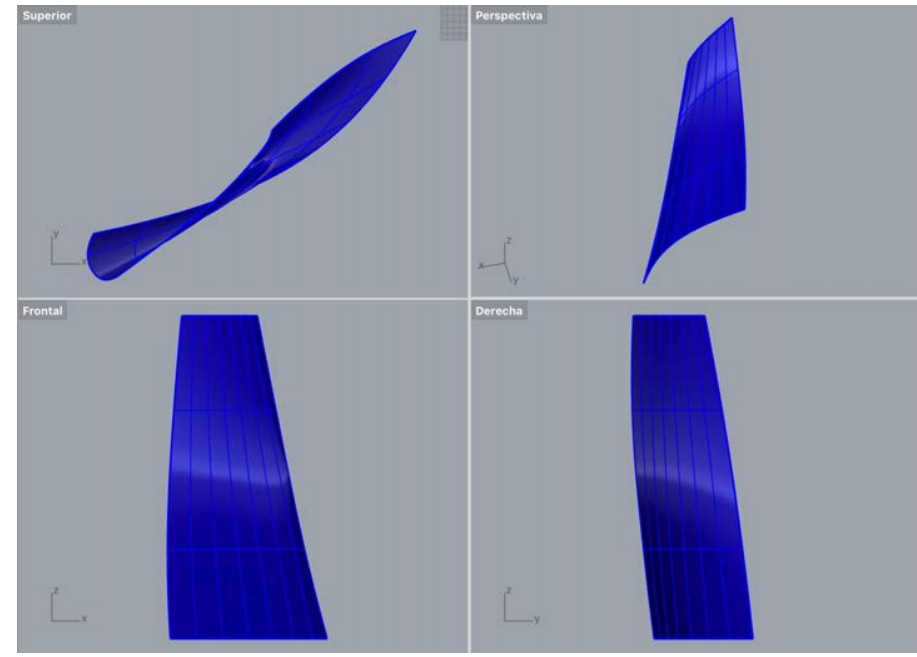

(b)

Figure 5.5: V-SPARS processed image (a) and preliminary three-dimensional (b) of a V-SPARS analysis for the Moth mainsail in a twisted flow at 45 degrees of AWA

When all the different shapes are collected (optimum trimmed flying shape) they could be introduced into the VR engine using procedural mesh elements (for example the dynamic mesh object in Unigine) that will be dependent on the AWA provided each time step by the time-domain VPP. This approach has the main disadvantage of only being visual and not allowing direct sail trimming capabilities interaction. With other configurations such as rigid wing-sails this procedure could be used for all the trimming situations because all the possibilities are based on determined basic geometric transformations of each of the wing elements. Different trimming elements positions could be reproduced using more parameters (wing sail camber, wing ele- 
ment position and rotation, wing element twist) into the VR engine procedural mesh generation and visual trimming indications are relatively straightforward from the integration point of view (even the deformed elements position of the wing sail due to the twist are well defined). An even simpler approach could be implemented if the wing sail elements are not deformed when twisting and trimming, and static mesh(involving simple geometric transformations) could be used instead of procedural mesh generation.

When a conventional sail has to be visually modelled to take into account all the trimming effects, the procedural mesh generation becomes very complex and a large amount of flying shapes have to be collected (in optimum and non-optimum trimming conditions) to be able to relate specific sail shape changes with specific trimming settings and changes.

An alternative to wind tunnel measured sail shapes is the generation of a complete sails aerodynamic model that includes wind and trimming parameters (different AWS, AWS, downhaul, mainsheet and mast bending positions, etc.) using precomputed data from a fast FSI package for sails, such as the North Sails Design Suite. The model interpolates sail shapes, forces and moments from the generated lookup table, containing the FSI generated data, for a specific AWS, AWA and trim parameters. This alternative offers enough level of visual accuracy for the sail shapes, but lower in comparison with the shapes generated with VSPARS or RANSE codes.

For a lower level of immersion and accuracy, a mixed approach could be used. The first approach (only dependant on the AWA and using the optimum trimming digitized flying shape) could be combined with visual trimming indications like the ones normally used in conventional sails (wool telltales presented in figure 5.6). Each visual trimming indication will be managed to switch from a specific position (oriented with the flow for a wool telltale) when well trimmed to another position when poorly trimmed (stalled situation for a wool telltale) based on VPP information. The main downside of these kinds of visual trimming procedures is that the aerodynamic forces and moments sail model inside the VPP has to be capable of taking into account the specific trimming parameters and generate a specific response. All the visual trimming indicators could be replaced with a trim to target visual system for the different trim variables indicating if the sail is well trimmed or not. 
All the described elements and approaches needed to achieve a certain level of visual trimming capabilities involve complex tasks and the results achieved could not answer the level of realism needed by the trimming crew. This is the reason why in some situations and simulator uses it is preferable to use a sail trimming autopilot.

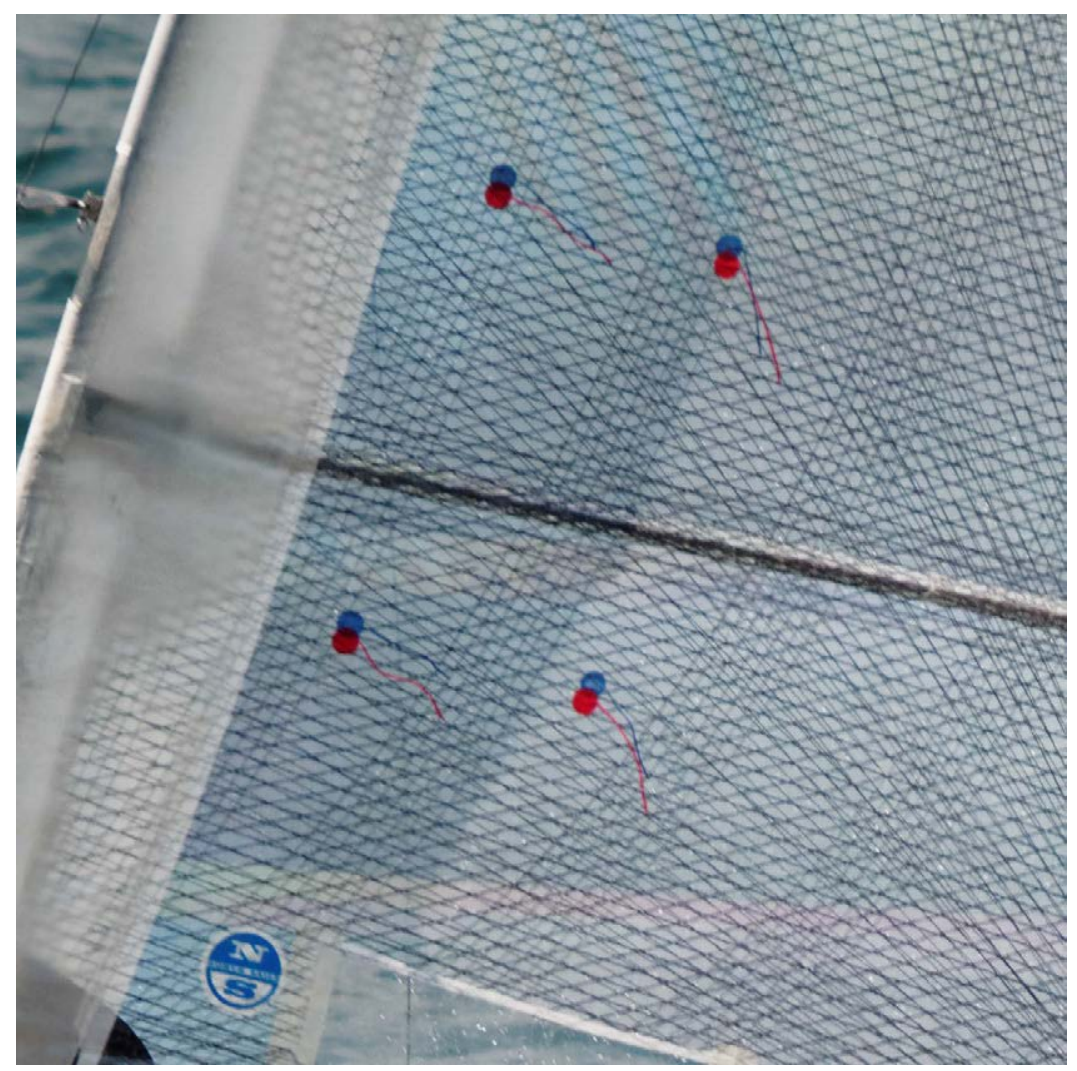

Figure 5.6: Wool telltales

\subsection{Virtual Reality Representation Systems}

As explained in chapter 4 , for a fully immersive simulator experience it is preferable to use a visual system that is able to isolate the user from the outside, avoiding the perception of external spatial cues that could degrade the kinematic motion cues generated by the motion platform. For a better visual experience, a closed environment should be used and the virtual surroundings have to be reproduced, far beyond the usual user vision field, to allow free head movement. In an aeroplane simulator or other closed cockpit vehicle, the field of view is limited to the windows (where the visual system is used) and the interior. When changing to sailboats, the cockpit is usually fully open and ideally, the user has to have the ability to look in 360 degrees around himself (in a regatta the sailor has to be able to look around to see the com- 
petitors). There are two main solutions to face that problem, to use a multi-display (or a curved one) setup or a VR Headset. The technical aspects of each technology will not be discussed because it is considered out of the scope of the thesis but the advantages and disadvantages of each technology related to the sailing boat Simulator will be discussed. Both technologies are normally compatible with modern VR engines and could be both adopted easily and fast depending on the task to develop when using the Simulator.

Multi-Display Systems: Usually based on the combination of three or more display or projectors it is the technology used in closed simulators. It allows reproducing large field of view using multiple projection or representation devices (normally they are limited to a 180-degree field of view). There are different solutions:

- A simpler combination of front and side conventional displays using asymmetric projection.

- Multi-channel image generation based on multiple PCs and projectors synchronized to generate different view-ports:

- Using multiple separated screens.

- Using continuous or curved screens with non-linear image correction and multi-projector edge blending.

- Fully immersive CAVE system (described in[35]).

The integration with all these different technologies usually combined with stereoscopic imaging is normally found in modern simulator-oriented VR engines such as Unigine [31], the one selected for the thesis. The main advantage of such setup is the capability of generating a fully immersive 360-degrees visual projection in closed environments. This allows to even introduce inside the simulator real physical elements such as a mock-up of the deck of the boat or even a real hull with its deck gear (for example when dealing with dinghy simulations as it was used in the VS-1 simulator [12]) allowing direct physical interaction between the user and the Simulator. This option will improve user spatial awarenesses because the subject will be placed inside and use the same elements of the actual boat. The main disadvantages are the price and the amount of space needed. If a motion platform is used sometimes it is difficult to mount a fully 360-degrees visual system because of the weight and space limitations and the need of running a significant amount of cables on top of the moving platform. 
Virtual Reality Headset: There is a compact alternative to the expensive multidisplay systems, the Head-mounted display (HMD) or VR Headset. This device was first developed by Sutherland in [167]. It presents different advantages:

- It can reproduce the complete 360 degrees surrounding scene with only one device (based on head's spatial position).

- It is compact.

- There is no need for a multiple-PC rendering configuration.

- It is one of the cheapest alternatives.

- It allows user head and position tracking within the device.

One of the main disadvantages of such technology was the weight of the device affecting the user comfort. Modern VR headsets, such as the Occulus rift ([125]) or HTC Vive ([32]) solve that problem even integrating an advanced head tracking position and specific hand controllers. Another main disadvantage is that the user is completely isolated from every external physical item. This is the reason why it is more complicated to combine such visualisation device with a boat deck mock-up or real deck gear. The interface has to be adapted and usually has to be simplified or specifically developed for the user because it will be only aware of the physical elements around him by touch when wearing the headset. If the input simulator operation device is always in contact with the user hands (helm wheel, tiller) the problem is partially mitigated (is only suitable when the user remains sailing in the same tack). Another disadvantage in comparison with the multi-displays systems is that it offers a more restricted field of view. This kind of device is more suitable then for using the simulator in zones with limited available space or when a limited user operation is needed (Design process and testing, specific visual cues training, etc.). For a fully immersive visual, spatial and tactile experience the more expensive multidisplay alternative is preferred. There is a similar technology called Binoccular Omni Orientation Monitor (BOOM) that offers a wider field of view but requires the user to hold the device with their hands, making it useless for the Simulator application. A review of the different technologies explained made by Latoschik could be found in $[95]$. 


\section{Chapter 6}

\section{The Physical User Interface}

\subsection{A User Interface to Allow the Simulator Op- eration}

The time-domain VPP can be fully controlled using autopilots, but in such situation, it cannot be considered a Simulator. It is just a tool suitable for analysing, from the performance perspective, the boat dynamic behaviour. It is a limited tool in comparison with the Simulator which adds a new layer of usability and extended capabilities. The main advantage regarding such layer is the possibility to adapt it to any needs that can arise during the use of the Simulator. For instance, for simple tasks, a simple user interface can be used (even just based on a basic controller such as a keyboard). For complex situations (very reactive boats, high-level training, detailed performance tuning) the complexity of the interface can be raised to fulfil the specific needs of each situation and usage (using steering elements, realistic sail controls, deck mock-ups, etc.). When dealing specifically with advanced training with high-level athletes, this Simulator component becomes a fundamental part. There is an increasing opinion in the simulator scientific community that the full flight simulators are not really necessary for early training stages of novice pilots (in favour of lower fidelity simulators) as explained by Stewart et al. in [165]. They also pointed out that "experts require (and prefer) higher levels of fidelity" and also that when talking about expert pilots "it is not new learning per se but assessment and refreshment of skills which have already been mastered". From the sailing boat Simulator perspective, high-level athletes can be considered as expert pilots and require the same level of fidelity including a realistic operation interface and cockpit and deck layout reproduction. In early stage training or specific design and performance assessment tasks, a simplified interaction layer can be used in order to facilitate the learning curve to new equipment and configurations, 
and simplicity of use.

The adaptability is also enhanced with the use of the VPP autopilots that can take care of specific tasks (Trimming autopilots, steering, etc). This allows reproducing specific situations and developing and analysing the specific performance, actions chain and decision-making process of each individual crew member. It improves the time usage of the machine and avoid unnecessary economic expenses (bigger platforms, the use of multiple platforms at the same time for different crew members, etc.).

Before each time step calculation within the time-domain VPP, the interface sends all the settings and adjustments changed by the user in order to update their values inside the VPP for the immediate time step calculation. For a general overview of the physical user interface implementation within the simulator please refer to the diagram in Figure 2.1.

\subsection{From a Simple Control to a More Realistic Ex- perience}

As explained before, in some situations the interface between the user and the simulator has to be adapted to fulfil specific requirements (that can be related to the simulator use, space available, economic resources, boat design stage, etc.). There are some specific situations where the rest of the hardware responsible for the other user inputs during the simulation is the element that will define the proper way to build and design the user operation interface. In chapter 5 two visualisation system were presented, the multi-display system and the VR headset. The multi-display setup allows any kind of configuration that can fit inside the space surrounded by the display (for example the complete cockpit in aeroplane simulators or a car inside a driver simulator), giving the highest flexibility. For such type of setups, the only decision in which the simulator team is involved is to choose the level of fidelity that is wanted for the user in term of spatial perception and operation. However, the VR headset does not allow the same approach because of the way the VR visual technology is implemented. In fact, when using a headset, the field of vision of the user is completely covered, the spatial awareness of the physical elements surrounding him is replaced by the space represented visually by the VR engine. A normal interaction between the user and the physical elements around him is not possible. The only 
sense that the user can use to reach and manipulate any object closer to him is the touch. Usually in seated cockpits, even in no-vision situations, steering and control devices can be easily reached by hand because they are fixed in a certain known position (this is the case for a steering wheel in a car or for the pedals in a plane or in a car which are normally used only by the feel of the feet with the pedal with no vision involved). This can be only applied to the steering elements (wheel or tiller) and for some trimming devices (only if they are held by the hand constantly) for boat simulations sailing always in the same tack (almost fixed user position). A solution that allows an increased level of interaction (for example adjusting more trimming and foils settings onboard) is proposed by the manufacturers of the different headsets. This solution consists of a pair of ergonomic hand-held controllers with buttons and a hand tracking position system (figure 6.1). The main inconvenience with this solution is that even if it allows an increased level of the virtual boat operation, it will not help to improve cockpit spatial awareness and deck and onboard elements familiarity of usage, resulting in poorer quality advanced training and overall experience.
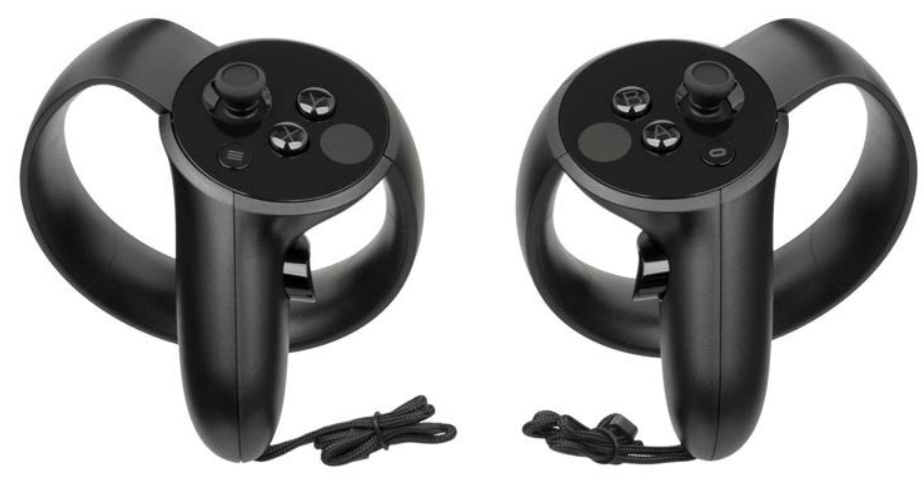

Figure 6.1: Occulus Rift Touch hand-held controller. Photograph by Evan Amos.

Source: https://upload.wikimedia.org/wikipedia/commons/d/d8/ Oculus-Rift-Touch-Controllers-Pair.jpg

Having noticed the problems related to other simulator components, the different elements that can be used to operate the virtual boat will be presented. 


\subsubsection{Steering Elements}

To reproduce the steering devices realistically there are three main interaction layers that have to be taking care of. For an improved sense of fidelity, the shape of the steering element has to be reproduced exactly as it is present in the boat. This task is usually simple because a spare of the actual boat steering can be adapted easily for that purpose. The second layer that has to be reproduced is the one related to the mechanical behaviour of the steering element. This task is normally solved using an angular encoder to know the steering element angular position. The mechanical system that will translate this angular position into the rudder position can be easily modelled because it exists a direct relation between both positions. The last layer to be reproduced is the moment feedback transmitted by the steering element on-board related to the lift and drag forces generated by the rudders. This can be accomplished using a brushless electric motor that can generate a specific torque. In fact, the VPP calculations involve the force and moments calculations of all the appendages, such information can be directly transformed into steering torque information and fed into the electric motor. The main advantage of using the described elements is that normally brushless motors (Figure 6.2) include with their controllers an encoder that provides the motor shaft angular position. The only operation when implementing such system when no buttons over the steering element are involved is to adapt the wheel or tiler on-board mounting system to the motor shaft. The communication between the motor and the simulator is normally done using specific protocols such as CANopen (a high-level protocol for CAN-bus described in [19]) that can be easily implemented. 


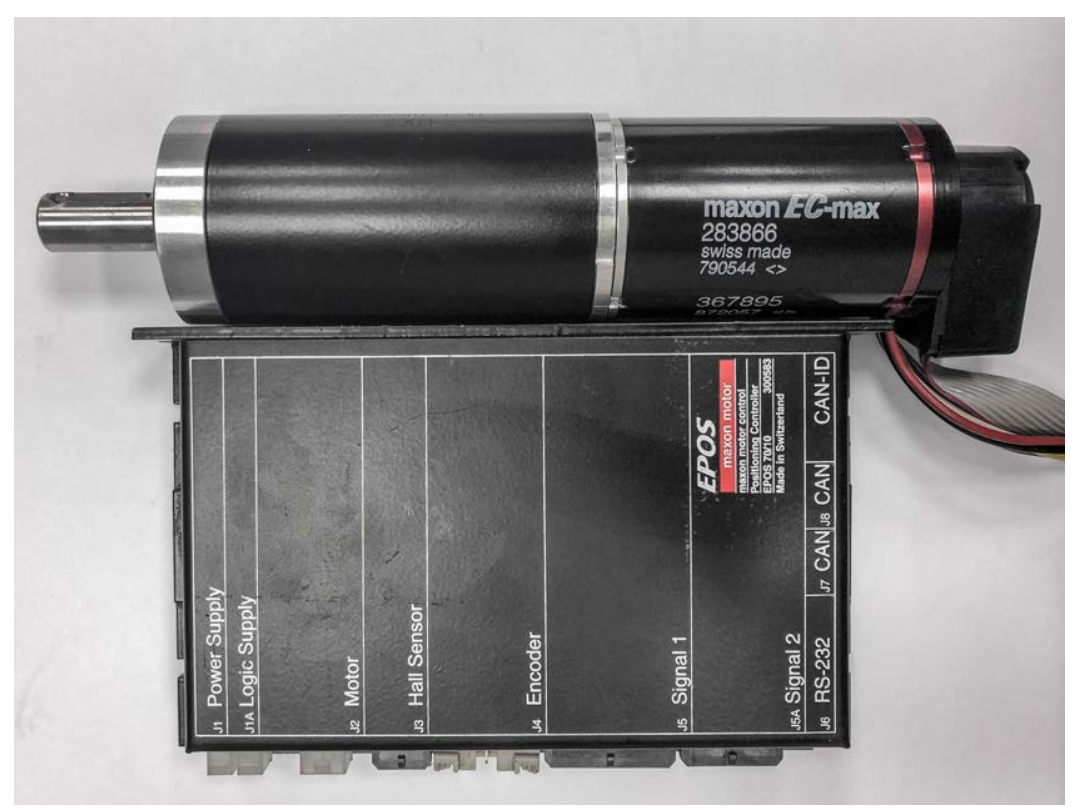

Figure 6.2: Brushless electric motor with controller and integrated angular encoder

In early design stages, for specific non-training usage and early stage training, simpler steering interfaces can be implemented, such as a joystick or a keyboard. The problem related to such interfaces is the limited amount of control and degraded sense of interaction with the virtual boat. For fast and responsive boats these problems are more evident and realistic steering elements are preferred.

\subsubsection{Sails Operation}

The same methodology applied to the steering elements can be applied to the sail controls. Sail controls are usually based on ropes and deck gear. Depending on the level of fidelity to be reproduced, it may be necessary to implement up to three interaction layers when dealing with rope based trimming devices. However, it is more complex to obtain a realistic result in comparison with the steering devices because they present a real behaviour that is more difficult to model. The three interaction layers cited before are the deck gear, the tension transmitted by the rope and the effect on sail trimming when the rope is "eased" or "trimmed". The tensions transmitted by the ropes can be obtained using simple analytic models (for example Harken offers simple load models for the genoa sheet and the mainsheet in [74]) that depend on basic data (mainly sail geometrical data and AWS) or using experimental data measured on board. If these models are combined with a brushless electric motor then, the calculated tension can be generated using the motor torque. The piece connecting the rope and the motor can be a rope drum and it is possible to calculate the amount 
of rope going inside or outside when trimming based on the motor angular encoder and the rope drum geometry. Tension reproduction is only necessary for the principal trimming elements which are normally submitted to higher tensions (mainsheet, jib sheet, etc.). For minor trimming elements (principally the ones that did not need winches or de-multiplication sets of blocks and runs directly through a cleat or rope clutch, such as the spinnaker pole outhaul, etc.) the tension mechanism can be simplified with a spring loaded one (pre-calculated based on experience or measured rope tension) and an encoder. The main challenge appears when trying to model the effects on trimming per amount of rope "eased" or "trimmed", this can be made using the user experience for parameter tuning, if he has already sailed the boat, or using measured relations between the sail trimming effect and the geometry of the running rigging, deck layout and deck and trimming gear.

It is recommended for high-level and elite athletes, in order to increase the level of fidelity, allow muscle memory training and improve user spatial awareness with the deck layout and gear usage, that all the ropes have to pass through the same elements (winches, blocks, cleats, clutches, etc.) present in the real boat and have to be positioned in the mock-up as they are or will be placed in the real boat.

When there is no need of trimming operation or the models implemented in the VPP relies on simplified trimming schemes (for example the de-powering and trimming scheme that can be found in the IMS aerodynamic model [33]), simple control based on simple buttons and switches can be used (or even a keyboard).

\subsubsection{User Position}

When dealing with dinghies, the weight and position of the crew directly affect the boat performance and behaviour (for larger boats this effect has to be also taken into account, but because normally only a part of the deck is reproduced, this effect can be reproduced using a specific force model inside the VPP). If a complete cockpit mockup is built, then the movement and position of the crew can be tracked using optical tracking devices. When using VR headsets, they have already built-in a head position (and sometimes also hands via the hand-held controller) optical tracking system, but it is more suitable for the head tracking for visual purposes than for full body location tracking. This type of technology is widely used in other fields such as movie making and animation (a review of the use of this technology in the film industry is presented in [20]), experimental motion measurements for research (for example it is used in offshore seakeeping research [192], sport research [146] and unmanned aircraft system research [119]), etc. That is the reason why the industry has developed a standard 
protocol called Virtual-Reality Peripheral Network (VRPN), presented by Taylor et al. in [170], that simplify the communication between the tracking device and the VR engine (as it is the case of Unigine that implements natively VRPN) or the tracking device and the VPP directly. With the specific body part tracked information, the position of the centre of gravity of the user can be determined and fed into the VR engine and the VPP. There are various commercial solutions based on optical systems that fit this kind of application, for example:

- Optitrack, developed by Natural Point, Inc [121].

- PhaseSpace Impulse X2E, an optical system developed by Phasespace [137].

- PST Iris, an optical system developed by PS-Tech [145].

- Vicon Vantage developed by Vicon Motion Systems Ltd [101]

\subsubsection{New Interface Systems and Other Settings Adjustment}

The appearance of the foiling boats equipped with advanced control systems have allowed using faster but unstable foils configurations. New interfaces were developed to interact with the foils control system and to change settings on-board quickly and effectively in order to maintain boat speed. In fact, one of the reasons that explain Team New Zealand's victory in the $35^{\text {th }}$ America's Cup was the decision to switch from manual grinding to pedal energy generation. With crew hands being free to execute other tasks, they implemented a specific interface to operate the foil rake and cant in a faster and more efficient way. To improve the wing-sail operation, they also developed a full hydraulic system, eliminating the winches and operating all the trimming settings through a hand-held controller.

The simulator can be used to develop and test, in realistic conditions, the devices that allow interacting with all the different systems on-board. The layout and position of the buttons can be analysed and tested, before the boat construction, inside the simulator. The prototypes also have to be installed inside the simulator during training to help the crew to be familiarized and to improve their skills with these specific operation devices, as a complement to the on-the-water testing and training. One of the main advantages of being able to test such interfaces before being on the water is to avoid possible malfunction or low performance that can put at risk the boat and the crew integrity. The only disadvantage is that the operation interface position is linked to the boat's cockpit, so in order to exploit these simulator capabilities at least a partial cockpit mock-up has to be built. 


\subsubsection{Cockpit Reproduction}

From the elite athlete point of view, being able to reproduce the boat cockpit (deck mock-up) will help the crew to be familiarized sooner with the tools and the space they will use when sailing and competing. For dinghies, if the hull fits over the motion platform and inside the simulator cabin, it can be directly used for that purpose (as it was used in the VS-1 Simulator [12]).

The usage of a cockpit reproduction or a deck layout mock-up is also influenced in the sailboat simulator by the design and optimization perspective usage. In fact, modifications over the layout of the deck gear and ergonomic and practical studies can be carried out in the mock-up inside the Simulator (without modifying the structure of the real boat potentially increasing its final weight). From the safety perspective, advanced and innovative ideas can be developed using the simulator in a safer environment without compromising the boat and the crew integrity. 


\section{Chapter 7}

\section{Conclusions}

The goals of the thesis were to study the applicability of the concept of a sailing boat simulator in a high-performance sailing environment and to provide the framework that will aim to demonstrate a fully immersive, simulated foiling sailing boat experience.

In order to answer these general goals, the concept of the sailing yacht simulator has been discussed. The different components have been identified (time-domain VPP, motion platform, visualisation system and the physical user interface) in order to generate the different cues and stimulations required by the simulator user. The possible uses of this concept as the general performance tool within a highperformance design and sailing campaign have been studied. Different uses of the simulator have been proposed in order to achieve the highest possible performance of the designed boat. Different areas have been detected where this tool can offer a series of benefits in comparison with the more conventional tools and procedures used nowadays. A general modular approach has been proposed in order to adapt the tool to the specific needs during the whole design and training process.

Every component has been evaluated in order to understand its specific needs and to identify the different solutions available to develop the simulator framework that is provided all across this work.

The main component of the simulator, PASim, a 6-DOF time-domain VPP in charge of the physics calculation of the sailing boat, has been analysed exploring the different types of forces and moments models and sources available. Specific solutions have been proposed in order to answer some problems related to the sailing boat simulator. 
The motion platform component has been evaluated in order to select the right platform solution to generate the simulator kinematic cues by analysing the different kinds of boats and their own needs. A general approach has been proposed using a Stewart Platform covering the full range of possible sailing boats to be simulated. Platform kinematics and motion cue generation have been explored and an implementation offering different possibilities using washout algorithms has been proposed. In order to facilitate the washout algorithm tuning, a specific procedure has been proposed based on genetic algorithm optimization problems.

The visualization system cues have been analysed in order to answer the simulator user physiological needs in that area. A general solution has been proposed based on available technology to generate virtual worlds answering the simulator needs and the integration with the VPP. Specific issues and challenges have been analysed regarding the special nature of the vehicle and environment to be reproduced. Later, different virtual reality representation devices and configurations have been studied in order to know the advantages and disadvantages of each device when used within the simulator.

Finally, the different needs of the physical user interface allowing the simulation operation of the virtual boat have been studied. Simple solutions to solve the sailing boat physical operation needs have been proposed. The convenience of each one of implementation and usage of the different interfaces (boat steering, sails operation, user position, etc) has been discussed for the different modular configuration proposed covering the needs of the different design and training stages.

Contributions: Different contributions have been made during the thesis for each of the areas studied in order to answer the goals and the different problems that have appeared while developing the simulator framework.

Regarding the possible uses of the simulator within a high-performance boat design and sailing campaign, four main areas have been identified where it can provide a strategic advantage in performance, with specific tasks:

- The simulator has been identified as a new design asset allowing: advanced performance assessment, early testing of design features, two boat performance evaluation with only one real boat, etc. 
- It has also been identified as the perfect testing platform allowing: to be independent on weather conditions for training and testing, to evaluate boat limits safely, to test new deck gear, etc.

- It is also a new interface for improved communications between athletes and designers.

- And finally, it has also been identified as a sailor's training tool, allowing: race preparation, modelling of race situations, giving assistance toward a steeper learning curve, etc.

To improve flexibility when using the simulator to cover all the possibilities explored above, a modular approach has been described and proposed as the way to follow when implementing the simulator.

Regarding the time-domain VPP component:

- Suitable models and autopilots schemes for foiling boat VPP have been identified and proposed to offer different possibilities when reproducing the sailing boat physics.

- Different model corrections have been proposed to solve various problems related with the foiling boat physics such as the different time-domain corrections implemented, the piercing foiling calculation strategies, the methods implemented to improve computational efficiency, etc

- Various numerical schemes have been analysed showing that it is preferable to use higher order numerical schemes for foiling boats. Specific recommendations regarding time step and computational needs have been introduced.

- A selection of specific models for forces and moments have been generated and implemented for the Moth Foiler allowing future VPP development.

Multi-threading and multi-computer setups have been also proposed to reduce computation time and possible execution-time lag problems.

Regarding the motion platform: 
- A general approach for all kind of sailing boats has been developed including the use of a Stewart Platform combined with a cue generation module based on washout algorithms. The best washout algorithms have been identified for different boat types and situations.

- A specific washout parameter tuning scheme and algorithm have been implemented in order to facilitate the tuning task. The algorithm is based on genetic algorithms and can use motion data for training based on different sources. It can be adapted for the different uses of the simulator.

Regarding the visualisation system:

- The best suitable VR engine has been identified to answer the simulators needs and different solutions have been proposed to solve representation problems regarding sail cloth reproduction.

- The usage of the VR engine to generate environmental cues has been studied, proposing its use for generating sea state cues.

- Some innovative possible uses of that component of the simulator framework have been introduced in order to show vital information and to improve two boat interaction awareness.

Finally, regarding the user control interface:

- Simple solutions for the different elements present in this component have been developed based on available technology to facilitate later development and integration.

- Following the general modular approach for the simulator framework, a specific strategy for each control interface element has been developed.

All the contributions enumerated previously have been the elements helping to build the sailing boat simulator framework presented in this thesis.

Future work: The modular simulator framework has been defined in order to solve and fulfil all the problems and needs that have been evaluated and identified during the development of this work. The solution proposed aim to offer a fully immersive, simulated foiling sailing boat experience. But other ideas and further development have emerged, that has not been solved or addressed during the thesis, such as: 
- With the whole system physically built, testing and fine-tuning every system component while using the tool within a design and sailing campaign. Using feedback from designers and sailors to change, improve and correct the modular framework proposed in this thesis.

- New solutions have to be investigated, regarding the way the user will interact with the simulator, from the designer and the sailor perspective. This way, new visualization physical interface solutions can be proposed widening the application of the system.

- New systems and needs have to be identified to cover new specific design problems and innovative sailboat configurations such as the kite foil.

- A study has to be carried out to explore and identify if the simulator framework modular approach provided here can be expanded to different kinds of marine vehicles and the needs that this expansion will imply.

- The concept of simulator autopilots has to be further developed, studying new control techniques for multiple adjustment variables and objectives, in order to deal with dynamically complex boats. New uses and methodologies for autopilots need to be explored to increase simulator flexibility and capacity, as well as their use as a verification tool for the simulator operation itself. 


\section{Appendix A}

\section{AC72 Dynamic Data Time History Plots}

As indicated in section 3.4, the graphs show a time-domain simulation of an AC72 boat using different numerical schemes, under specific conditions of True Wind Speed (TWS $=7$ knots) and True Wind Angle (TWA $=130$ degrees $)$ and reasonable initial values for the adjustment variables (but without using a pre-calculated VPP equilibrium solution for such values). The initial boat speed was 11 knots and the boat was set sailing in displacement mode (not in foiling mode), behaving like a conventional catamaran. The BSP plots are also presented in section 3.4. 


\section{A.1 Time History Comparison for Different Nu- merical Schemes}




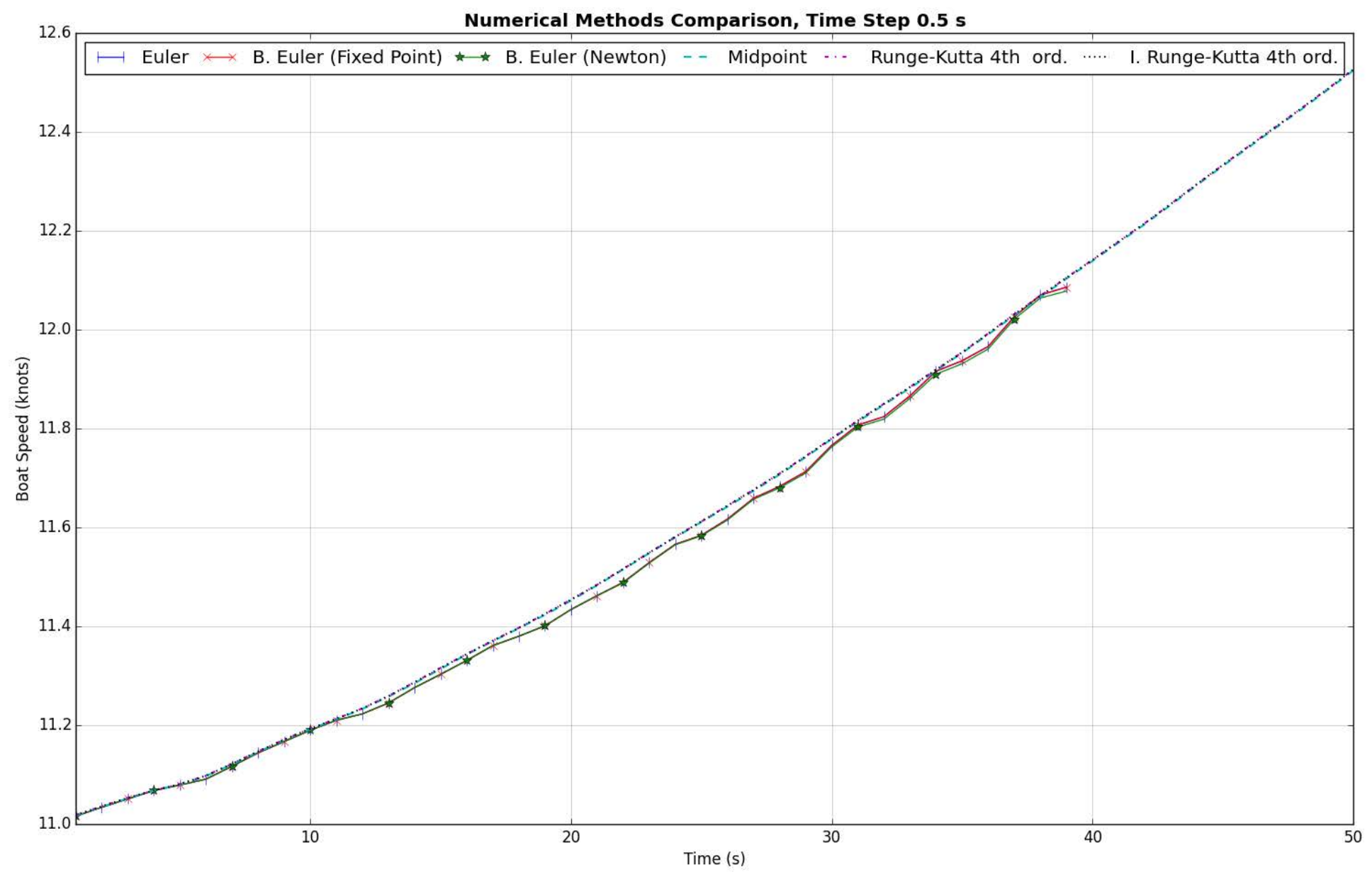

Figure A.1: Time history comparison for different numerical schemes - BSP 


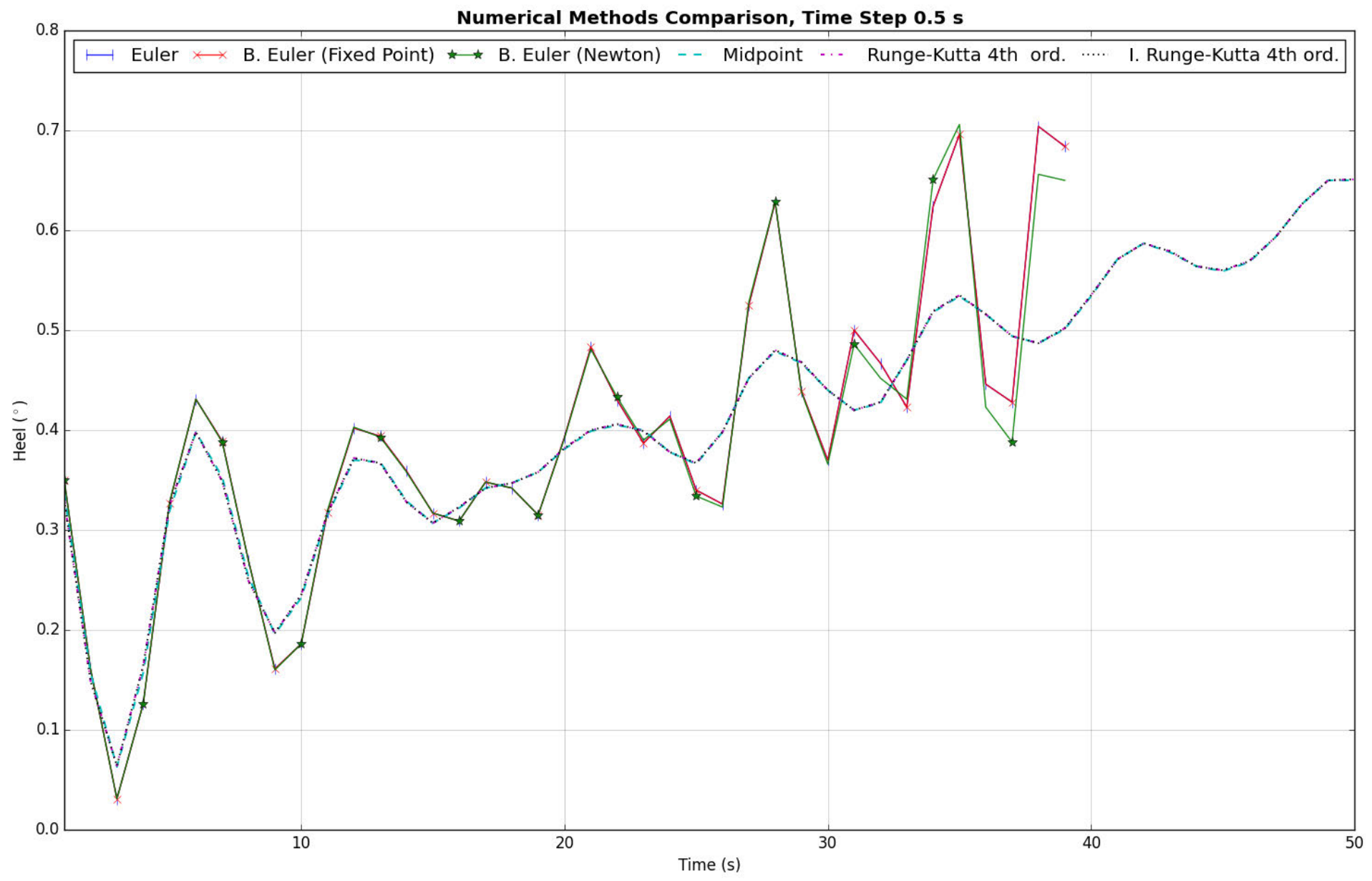

Figure A.2: Time history comparison for different numerical schemes - Heel 


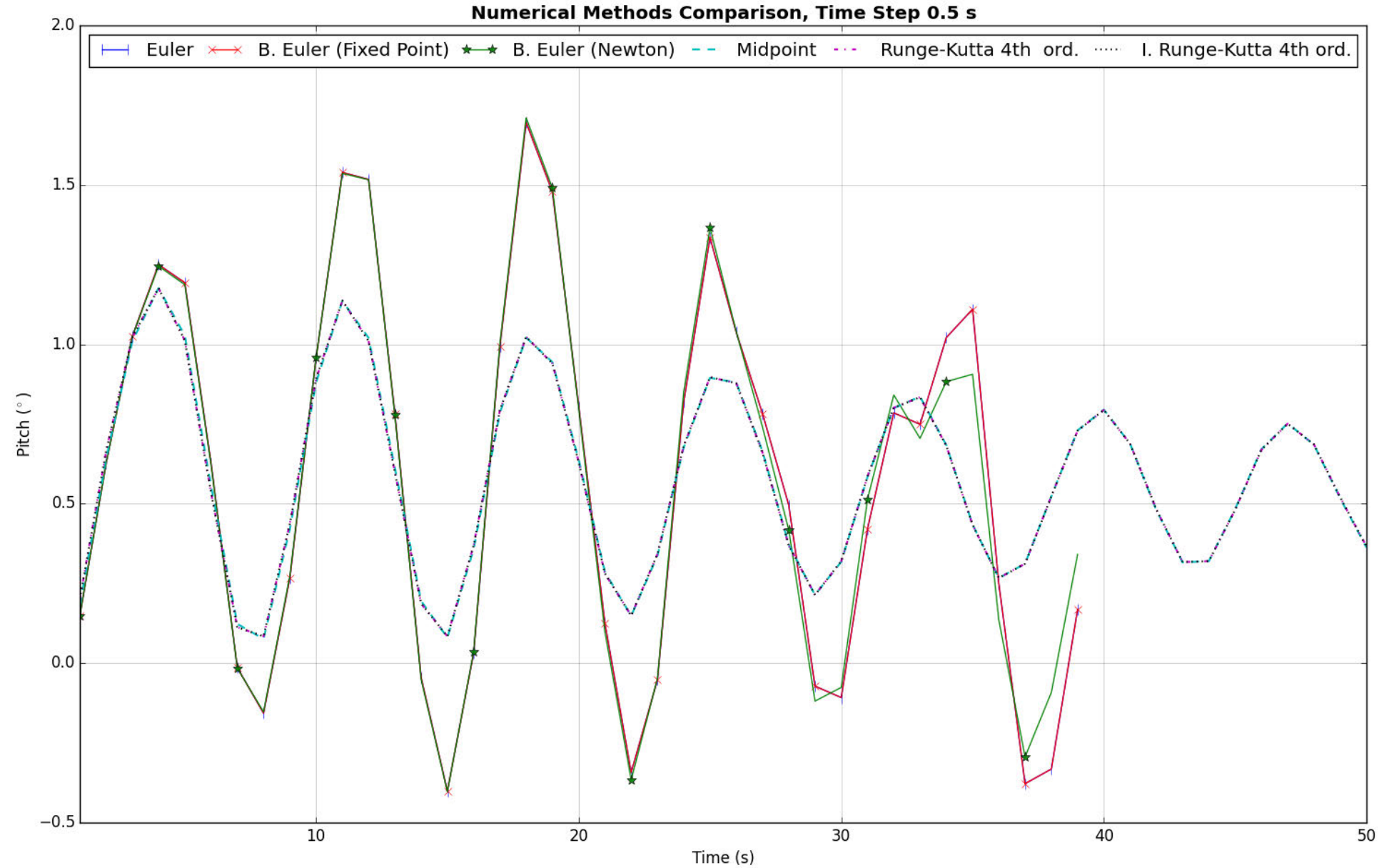

Figure A.3: Time history comparison for different numerical schemes - Pitch 


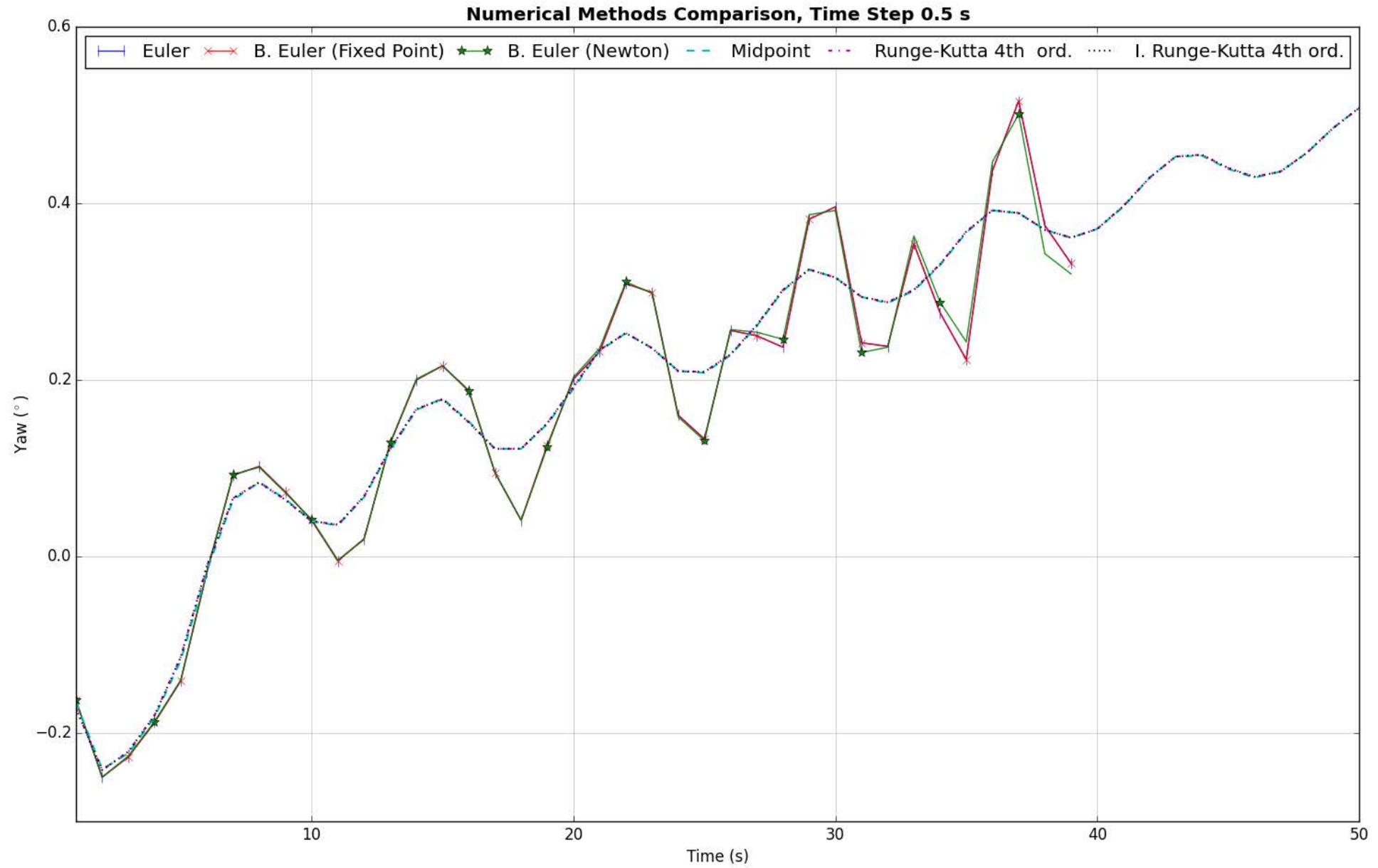

Figure A.4: Time history comparison for different numerical schemes - Yaw 


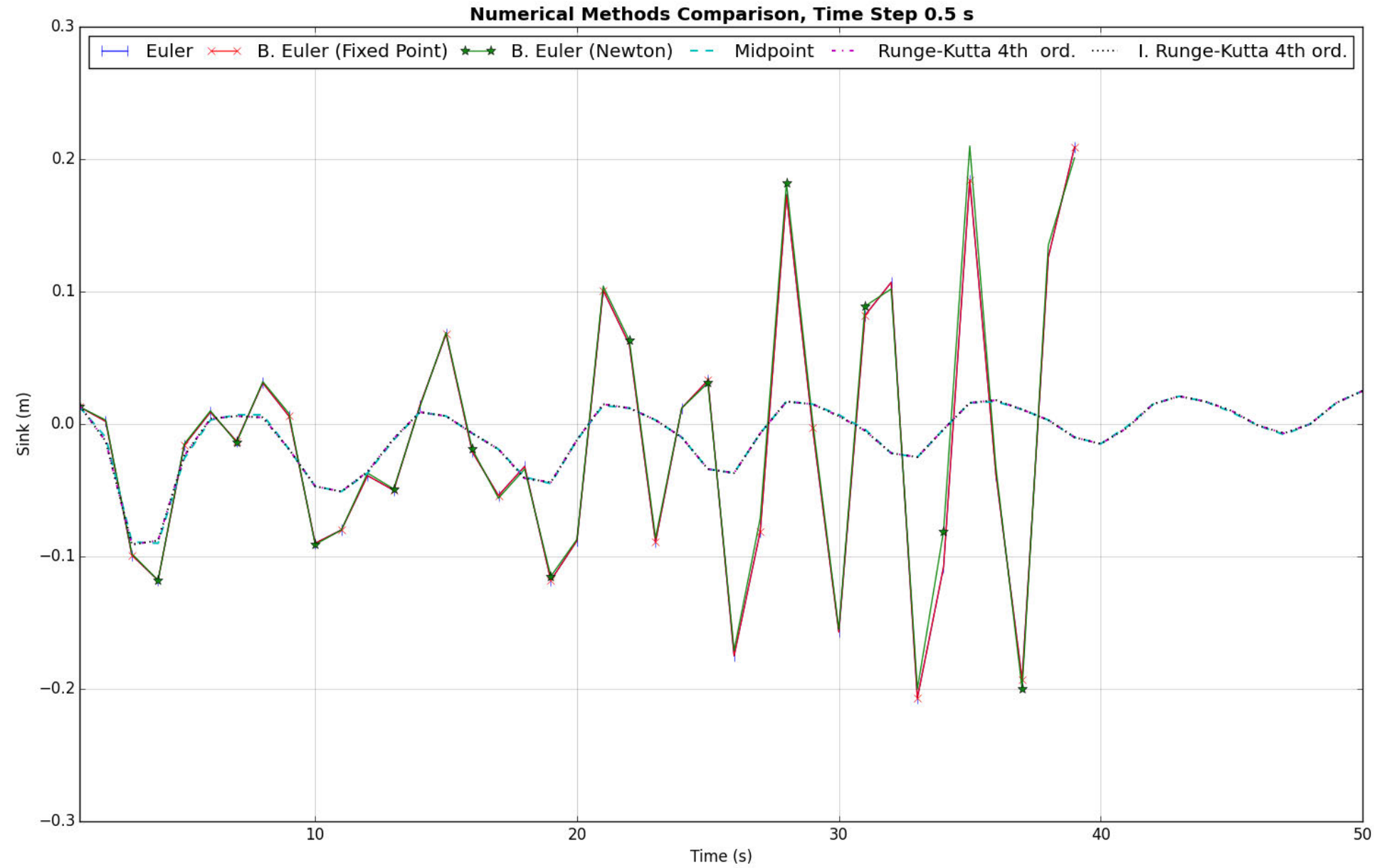

Figure A.5: Time history comparison for different numerical schemes - Sinkage 
Numerical Methods Comparison, Time Step 0.5 s

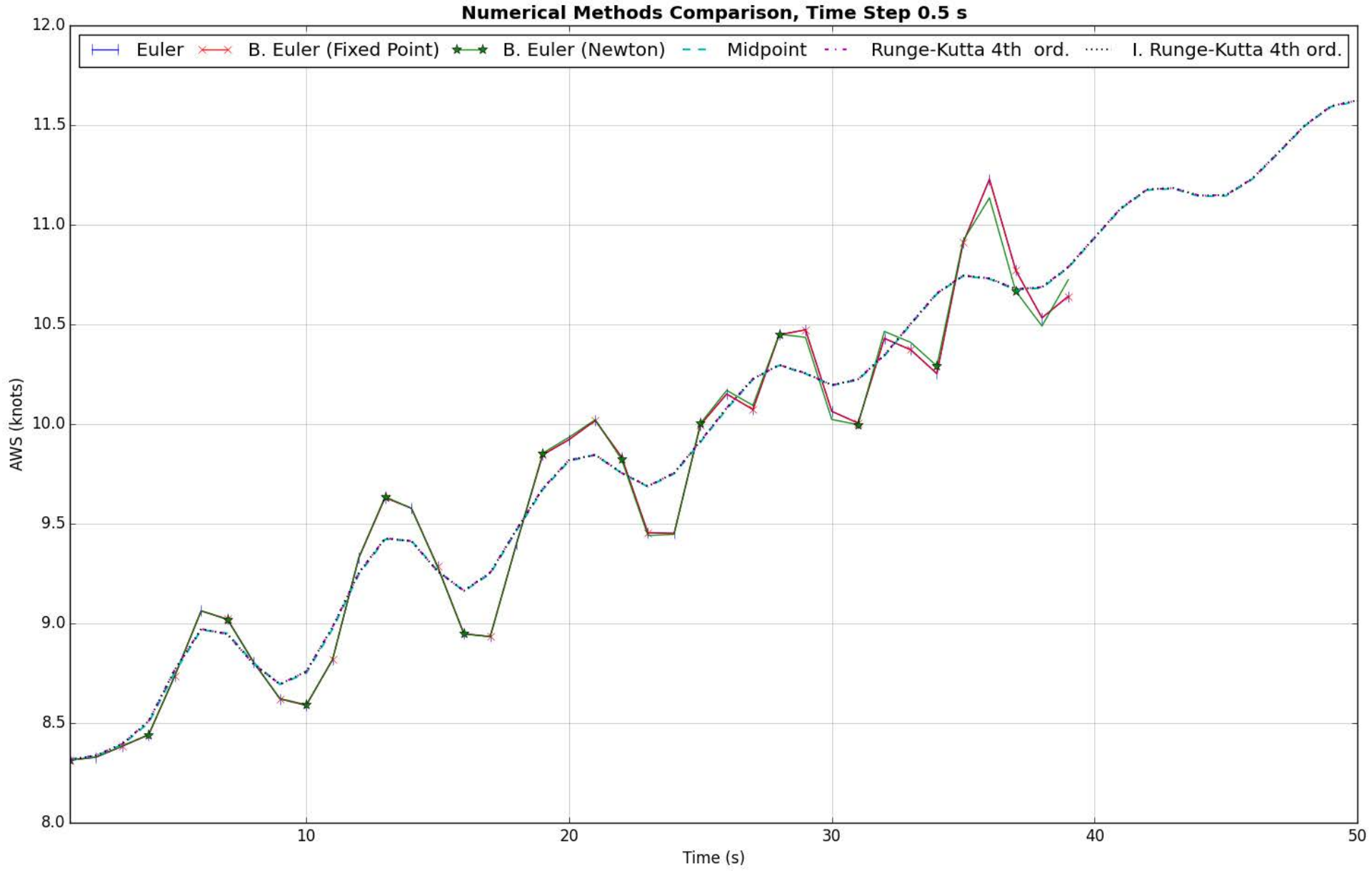

Figure A.6: Time history comparison for different numerical schemes - AWS 


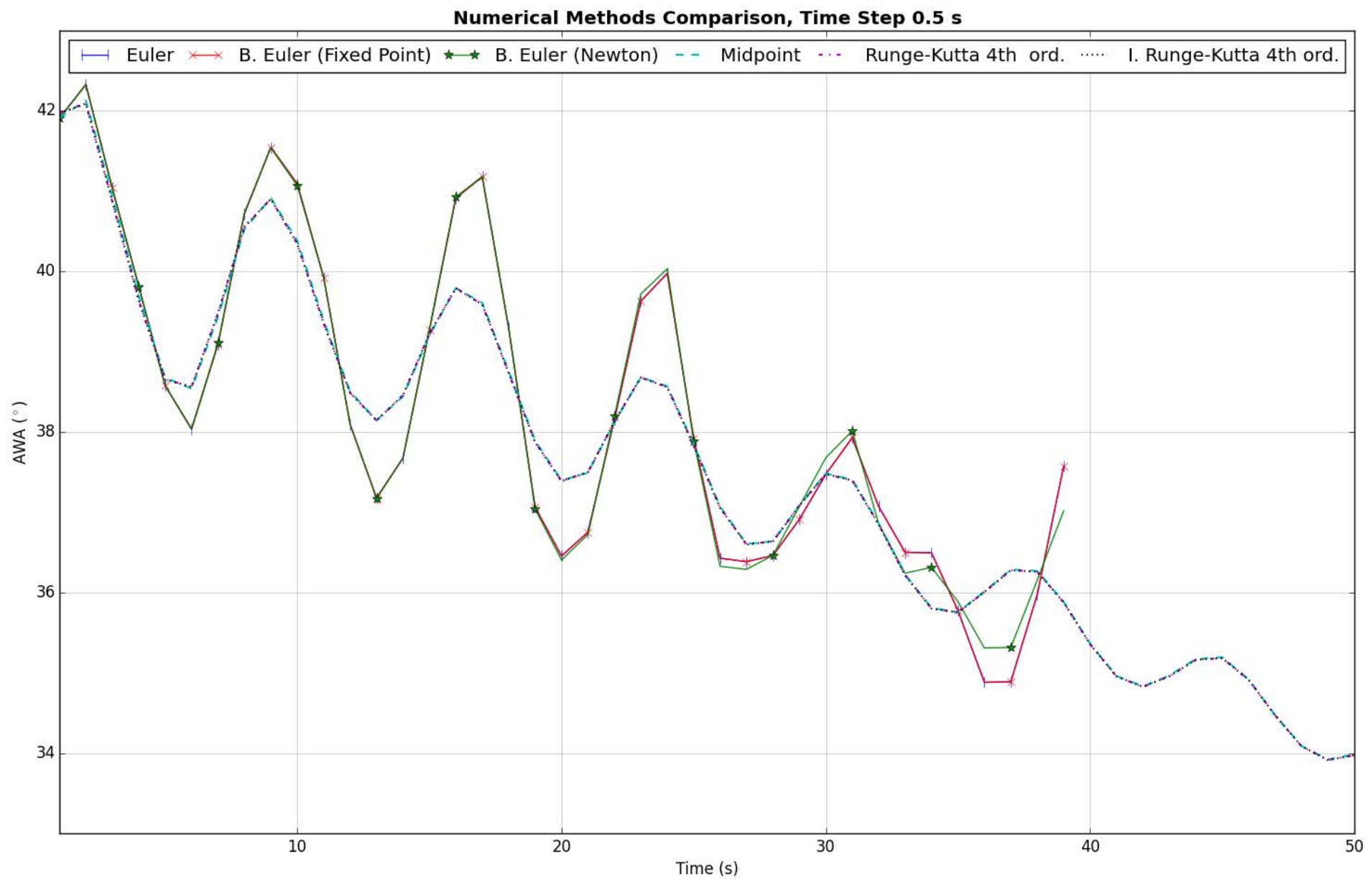

Figure A.7: Time history comparison for different numerical schemes - AWA 


\section{A.2 Time History Comparison Using Euler and Midpoint Methods with Different Time Steps}




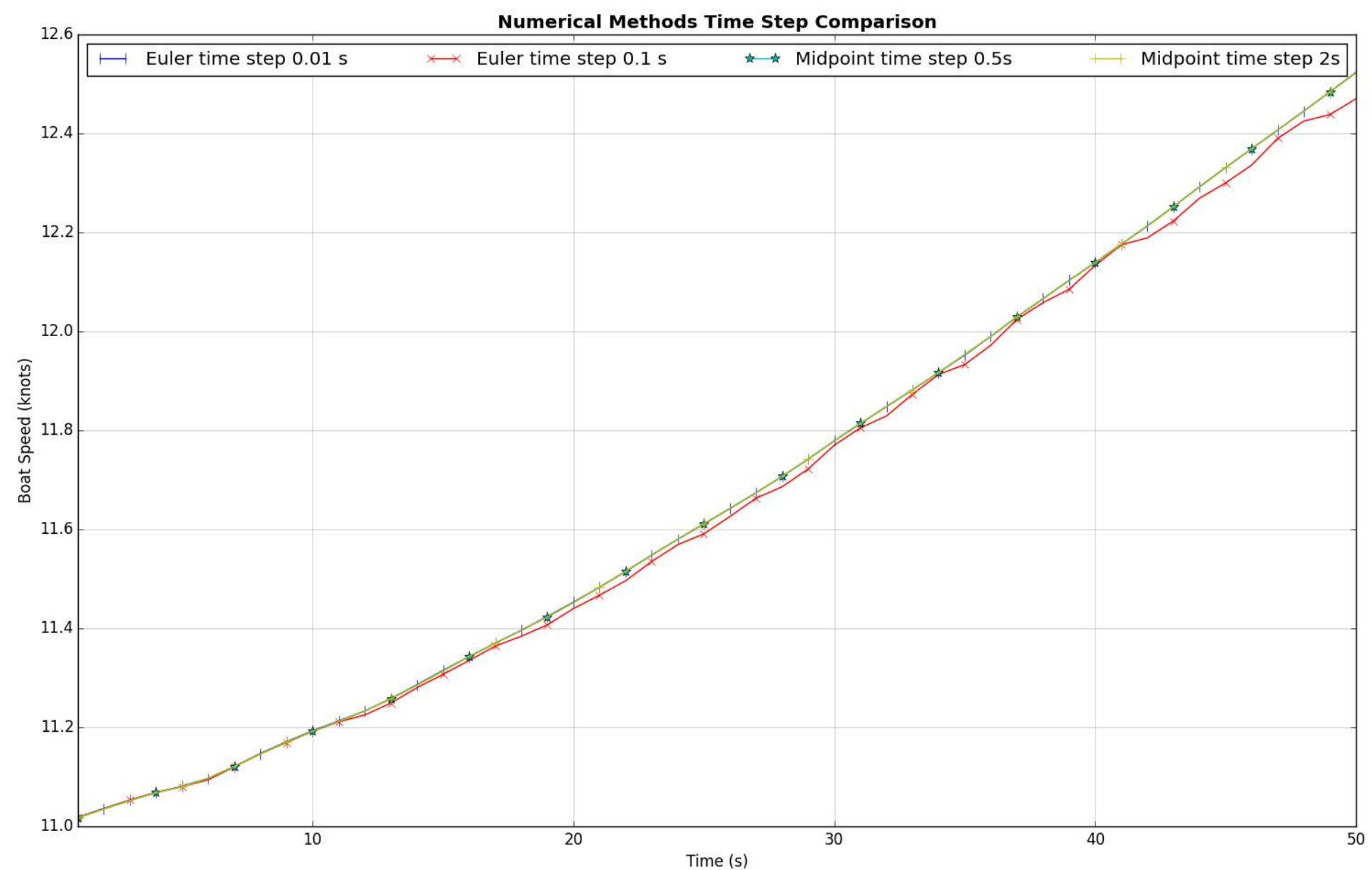

Figure A.8: Time history comparison using Euler and Midpoint methods with different time steps - BSP 


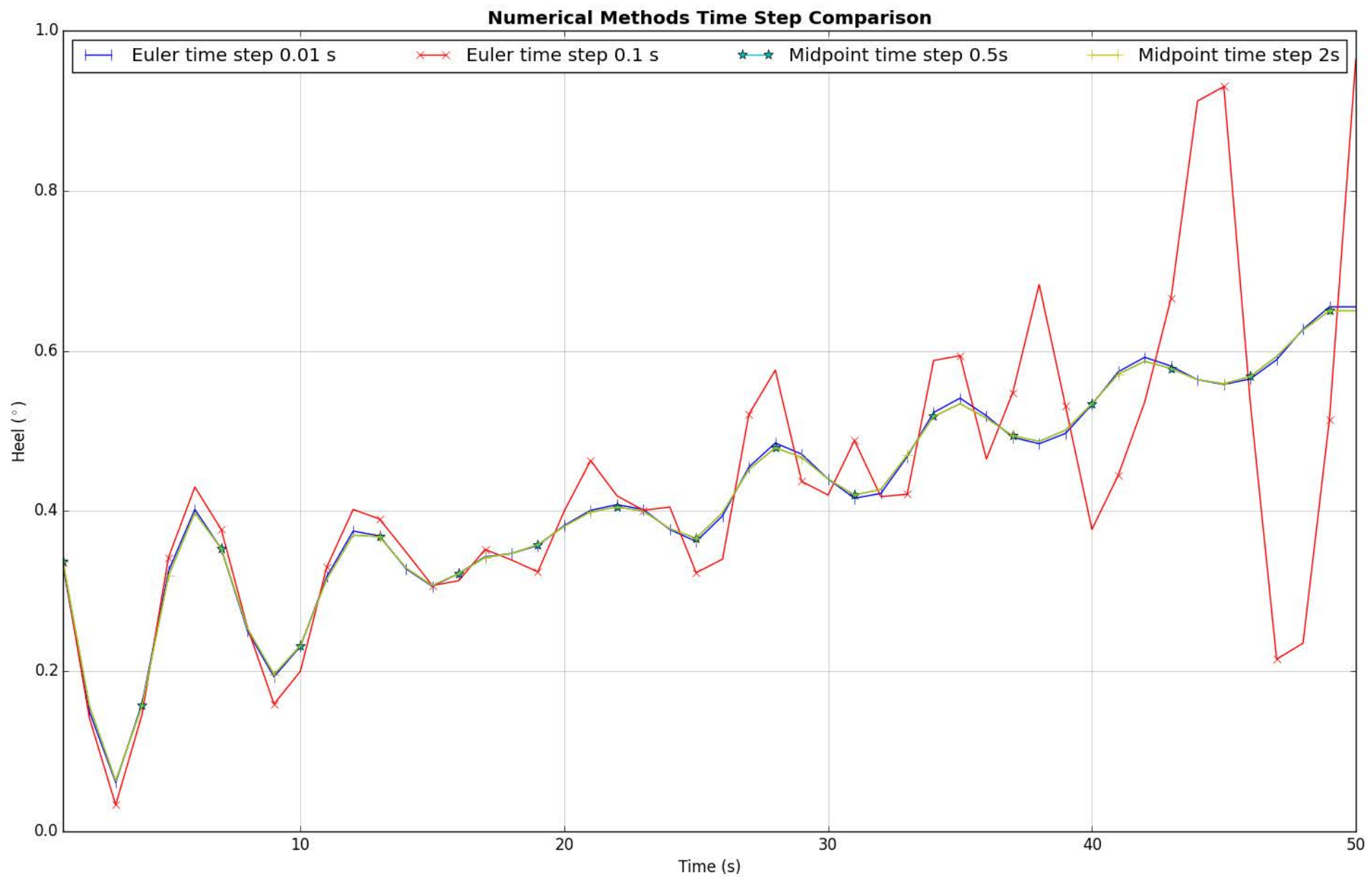

Figure A.9: Time history comparison using Euler and Midpoint methods with different time steps - Heel 


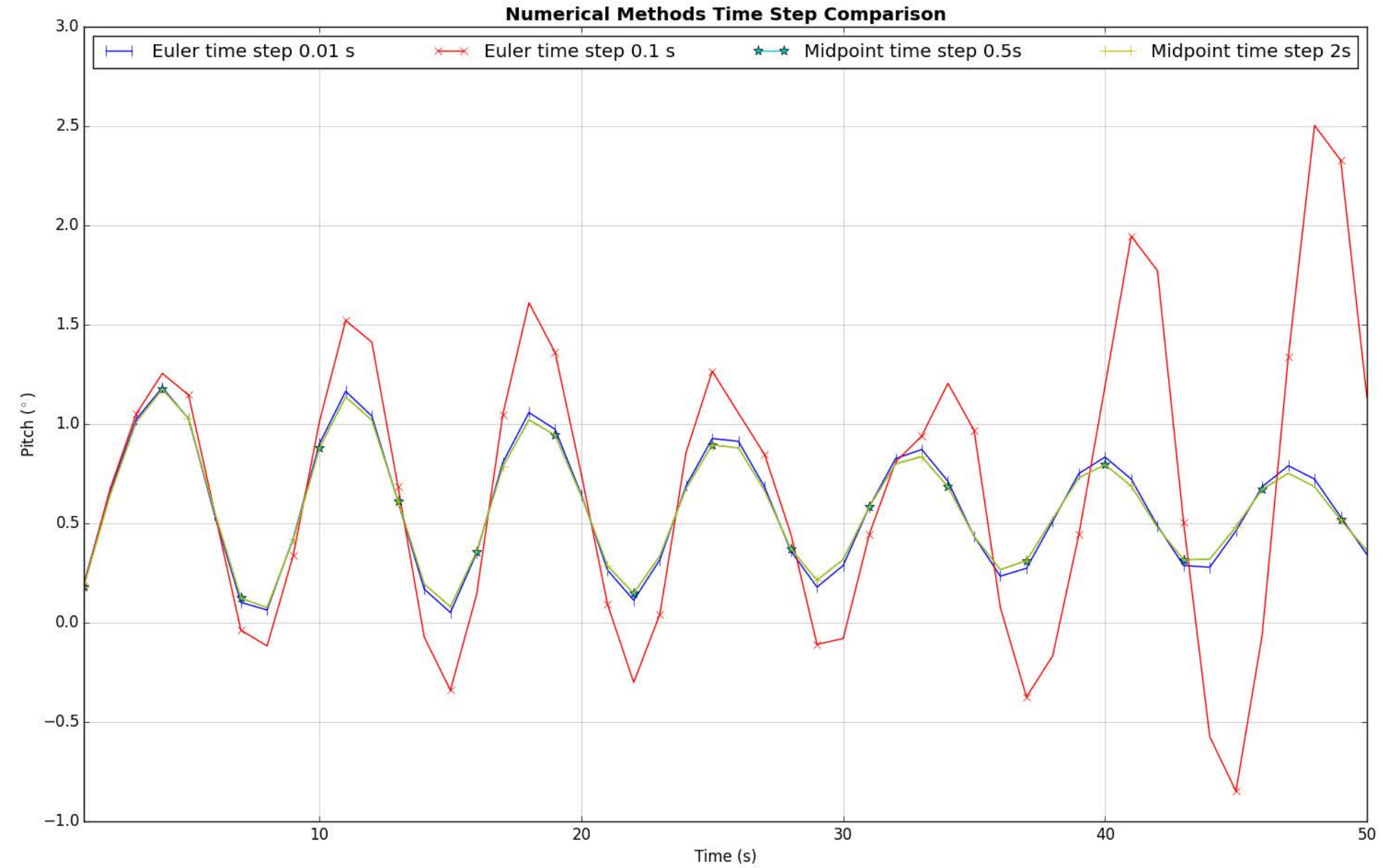

Figure A.10: Time history comparison using Euler and Midpoint methods with different time steps - Pitch 


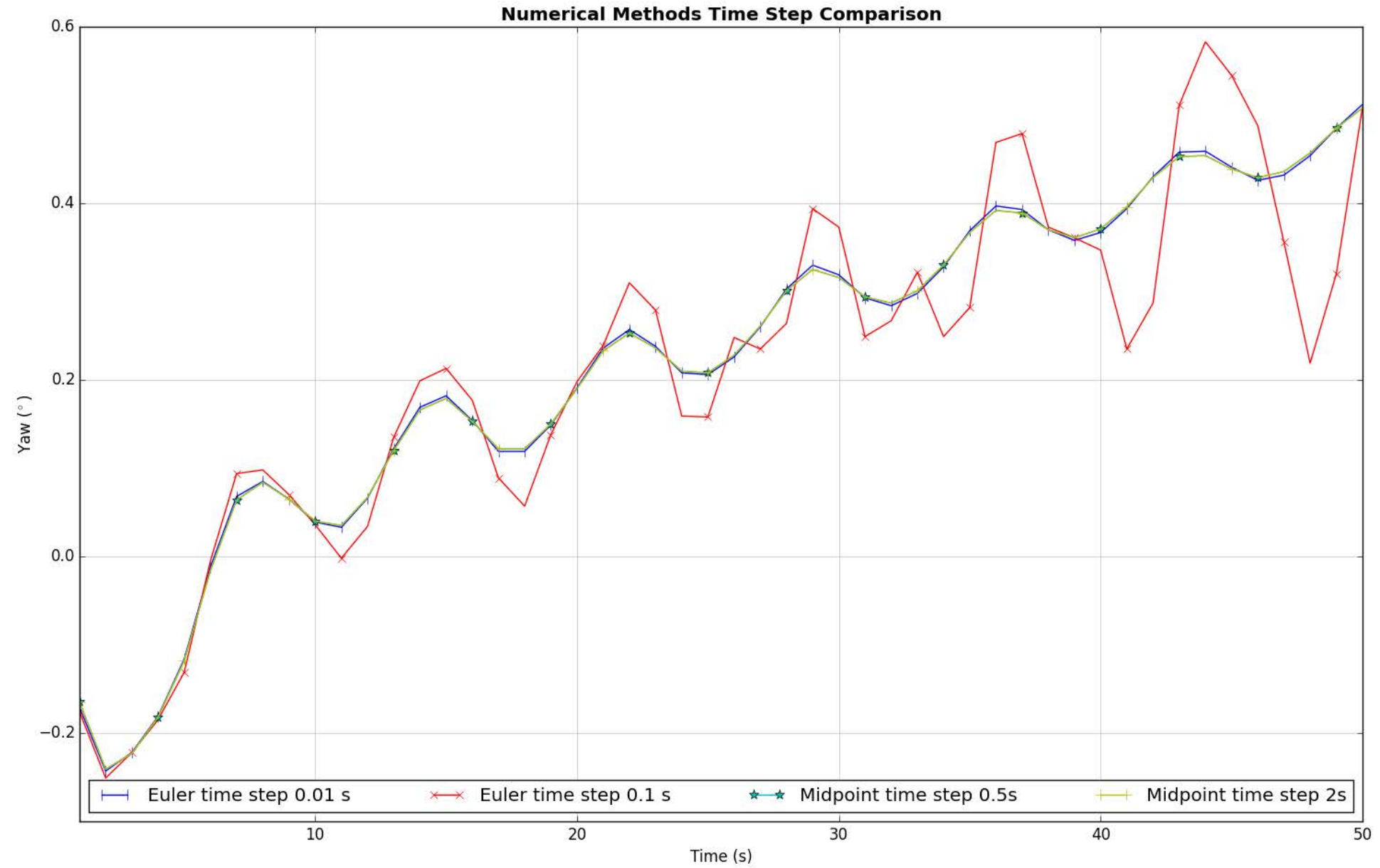

Figure A.11: Time history comparison using Euler and Midpoint methods with different time steps - Yaw 


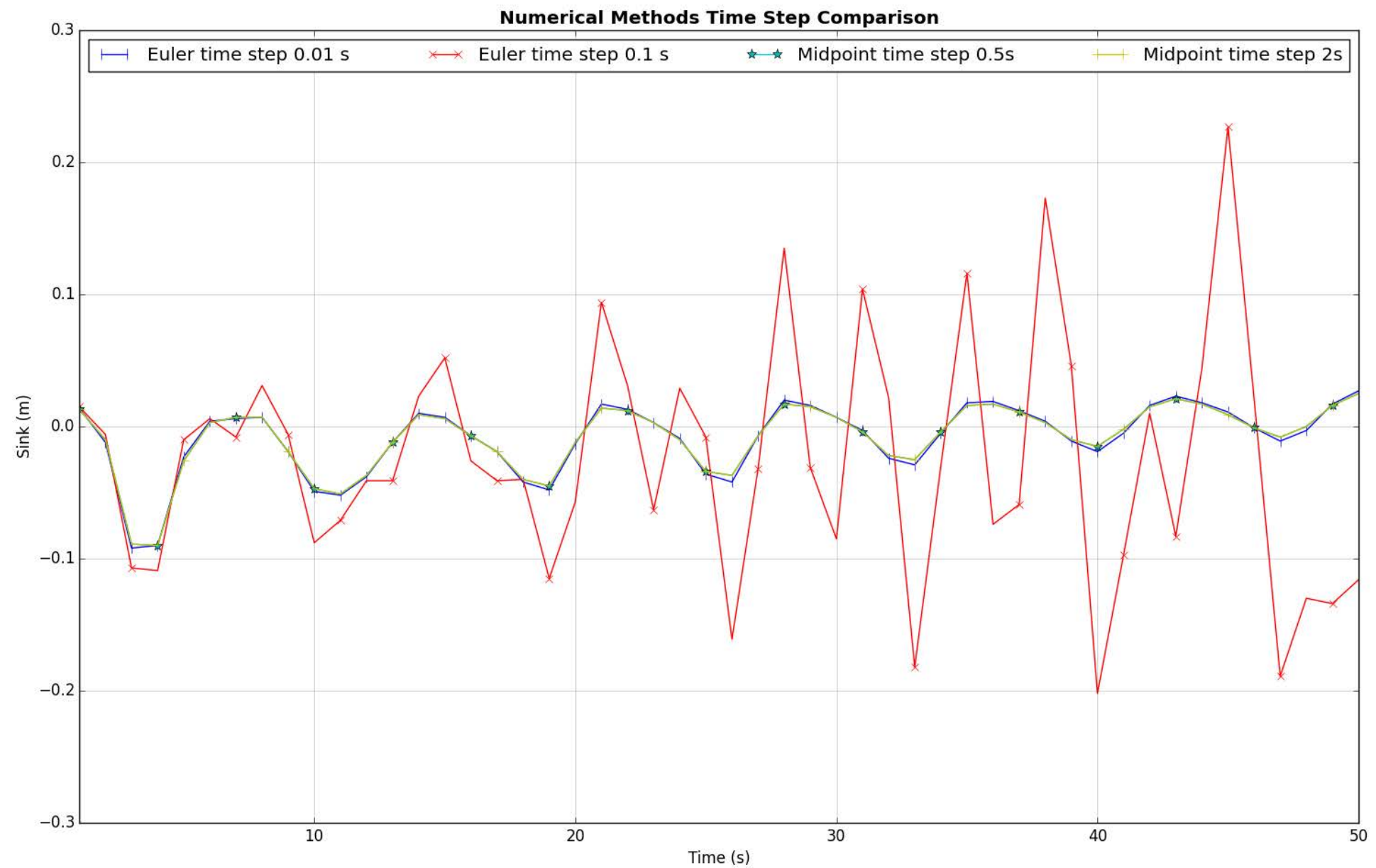

Figure A.12: Time history comparison using Euler and Midpoint methods with different time steps - Sinkage 
Numerical Methods Time Step Comparison

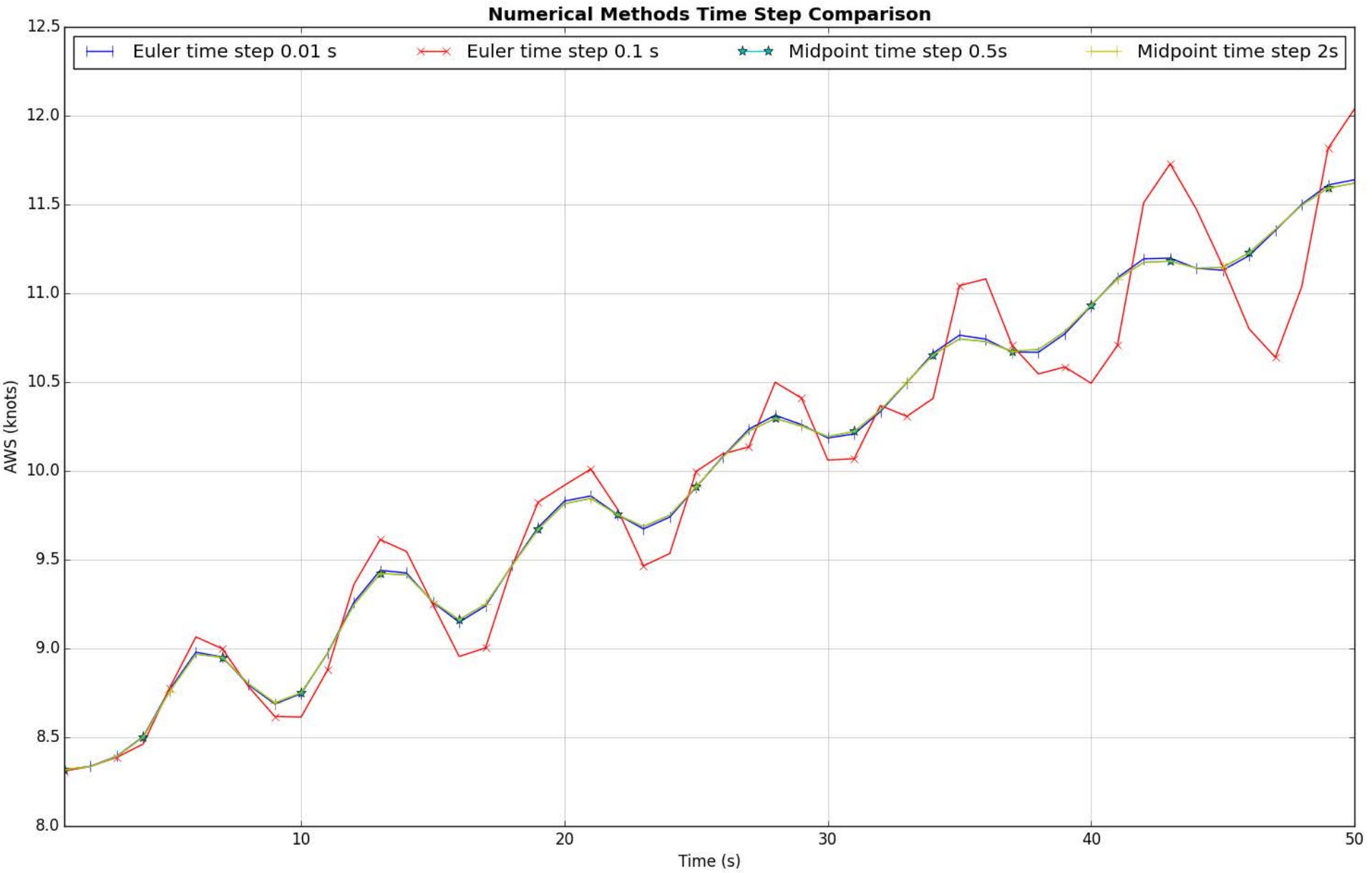

Figure A.13: Time history comparison using Euler and Midpoint methods with different time steps - AWS 


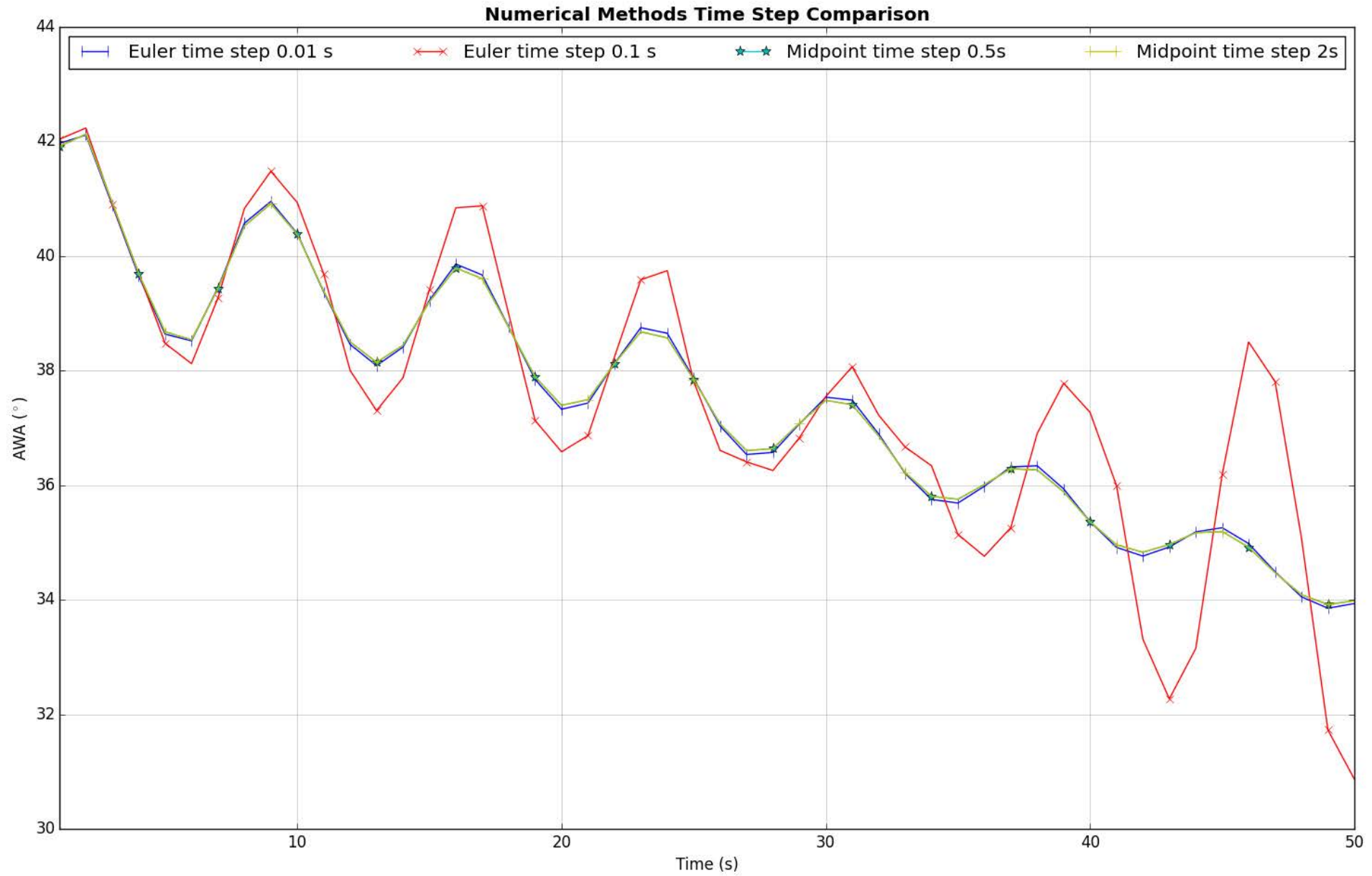

Figure A.14: Time history comparison using Euler and Midpoint methods with different time steps - AWA 


\section{Appendix B}

\section{Time History Data of the Simulated Foiling Moth Runs}

\section{B.1 Time History Data of the Simulated Foiling Moth Run Using a Heel PID Controller}

The following tables show the time history of some of the calculated variables during the Moth foiler time-domain simulation using the heel PID controller presented in section in section 3.5.6. 
Table B.1: Time history of the simulated foiling Moth run using a heel PID controller

\begin{tabular}{|c|c|c|c|c|c|c|c|c|c|}
\hline Time $(\mathrm{s})$ & $\mathrm{BSP}(\mathrm{kn})$ & Heel $\left(^{\circ}\right)$ & Crew Y Pos.(m) & Flat & TWS (kn) & TWA $\left(^{\circ}\right)$ & AWS (kn) & AWA $\left(^{\circ}\right)$ & $\mathrm{Mx}(\mathrm{N} . \mathrm{m})$ \\
\hline 0.0 & 8.407 & -1.426 & -1.250 & 0.868 & 8.000 & 70.000 & 13.442 & 34.004 & 0.000 \\
\hline 1.0 & 8.407 & -1.426 & -1.250 & 0.868 & 8.000 & 70.000 & 13.442 & 34.004 & 0.000 \\
\hline 2.0 & 8.407 & -1.426 & -1.250 & 0.868 & 8.000 & 70.000 & 13.442 & 34.004 & 0.000 \\
\hline 3.0 & 8.407 & -1.426 & -1.250 & 0.868 & 8.000 & 70.000 & 13.442 & 34.004 & 0.000 \\
\hline 4.0 & 8.407 & -1.426 & -1.250 & 0.868 & 8.000 & 70.000 & 13.442 & 34.004 & 0.000 \\
\hline 5.0 & 8.407 & -1.426 & -1.250 & 0.868 & 8.000 & 70.000 & 13.442 & 34.004 & 0.000 \\
\hline 6.0 & 8.407 & -1.426 & -1.250 & 0.868 & 8.000 & 70.000 & 13.442 & 34.004 & 0.000 \\
\hline 7.0 & 8.407 & -1.426 & -1.250 & 0.868 & 8.000 & 70.000 & 13.442 & 34.004 & 0.000 \\
\hline 8.0 & 8.407 & -1.426 & -1.250 & 0.868 & 8.000 & 70.000 & 13.442 & 34.004 & 0.000 \\
\hline 9.0 & 8.407 & -1.426 & -1.250 & 0.868 & 8.000 & 70.000 & 13.442 & 34.004 & 0.000 \\
\hline 10.0 & 8.407 & -1.426 & -1.250 & 0.868 & 8.000 & 70.000 & 13.442 & 34.004 & 0.000 \\
\hline 11.0 & 8.407 & -1.426 & -1.250 & 0.868 & 8.000 & 70.000 & 13.442 & 34.004 & 0.000 \\
\hline 12.0 & 8.407 & -1.427 & -1.250 & 0.868 & 8.000 & 70.000 & 13.442 & 34.004 & 0.000 \\
\hline 13.0 & 8.407 & -1.426 & -1.250 & 0.868 & 8.000 & 70.000 & 13.442 & 34.004 & 0.000 \\
\hline 14.0 & 8.408 & -1.427 & -1.250 & 0.868 & 8.000 & 70.000 & 13.442 & 34.004 & 0.000 \\
\hline 15.0 & 8.407 & -1.426 & -1.250 & 0.868 & 8.000 & 70.000 & 13.442 & 34.004 & 0.000 \\
\hline 16.0 & 8.407 & -1.427 & -1.250 & 0.868 & 8.000 & 70.000 & 13.442 & 34.004 & 0.000 \\
\hline 17.0 & 8.408 & -1.426 & -1.250 & 0.868 & 8.000 & 70.000 & 13.442 & 34.004 & 0.000 \\
\hline 18.0 & 8.407 & -1.427 & -1.250 & 0.868 & 8.000 & 70.000 & 13.442 & 34.004 & -0.010 \\
\hline \multicolumn{10}{|c|}{ Continue on next page } \\
\hline
\end{tabular}


Table B.1 - Continued from previous page

\begin{tabular}{|c|c|c|c|c|c|c|c|c|c|}
\hline Time (s) & $\mathrm{BSP}(\mathrm{kn})$ & Heel $\left(^{\circ}\right)$ & Crew Y Pos. $(\mathrm{m})$ & Flat & TWS (kn) & TWA $\left(^{\circ}\right)$ & AWS (kn) & AWA $\left(^{\circ}\right)$ & $\mathrm{Mx}$ (N.m) \\
\hline 19.0 & 8.408 & -1.427 & -1.250 & 0.868 & 8.000 & 70.000 & 13.442 & 34.004 & 0.010 \\
\hline 20.0 & 8.407 & -1.425 & -1.250 & 0.867 & 8.000 & 70.000 & 13.442 & 34.004 & -0.030 \\
\hline 21.0 & 8.408 & -1.429 & -1.250 & 0.868 & 8.000 & 70.000 & 13.442 & 34.004 & 0.050 \\
\hline 22.0 & 8.407 & -1.422 & -1.250 & 0.867 & 8.000 & 70.000 & 13.442 & 34.004 & -0.070 \\
\hline 23.0 & 8.408 & -1.432 & -1.250 & 0.868 & 8.000 & 70.000 & 13.442 & 34.004 & 0.080 \\
\hline 24.0 & 8.408 & -1.419 & -1.250 & 0.867 & 8.000 & 70.000 & 13.442 & 34.004 & -0.060 \\
\hline 25.0 & 8.407 & -1.434 & -1.250 & 0.868 & 8.000 & 70.000 & 13.442 & 34.004 & 0.000 \\
\hline 26.0 & 8.408 & -1.423 & -1.250 & 0.868 & 8.000 & 70.000 & 13.443 & 34.003 & 0.150 \\
\hline 27.0 & 8.407 & -1.419 & -1.250 & 0.867 & 8.000 & 70.000 & 13.442 & 34.005 & -0.400 \\
\hline 28.0 & 8.409 & -1.455 & -1.250 & 0.869 & 8.000 & 70.000 & 13.443 & 34.001 & 0.780 \\
\hline 29.0 & 8.406 & -1.365 & -1.250 & 0.865 & 8.000 & 70.000 & 13.441 & 34.007 & -1.250 \\
\hline 30.0 & 8.409 & -1.532 & -1.250 & 0.871 & 8.000 & 70.000 & 13.444 & 34.000 & 1.700 \\
\hline 31.0 & 8.406 & -1.272 & -1.250 & 0.863 & 8.000 & 70.000 & 13.441 & 34.006 & -1.890 \\
\hline 32.0 & 8.407 & -1.615 & -1.250 & 0.872 & 8.000 & 70.000 & 13.442 & 34.006 & 1.410 \\
\hline 33.0 & 8.412 & -1.250 & -1.250 & 0.865 & 8.000 & 70.000 & 13.446 & 33.993 & 0.360 \\
\hline 34.0 & 8.397 & -1.482 & -1.250 & 0.865 & 8.000 & 70.000 & 13.433 & 34.029 & -4.320 \\
\hline 35.0 & 8.426 & -1.652 & -1.250 & 0.881 & 8.000 & 70.000 & 13.458 & 33.960 & 10.710 \\
\hline
\end{tabular}


Table B.2: Detailed last 4 seconds of the simulated foiling moth run using a heel PID controller

\begin{tabular}{|c|c|c|c|c|c|c|c|c|c|}
\hline Time (s) & $\mathrm{BSP}(\mathrm{kn})$ & Heel $\left(^{\circ}\right)$ & Crew Y Pos.(m) & Flat & TWS (kn) & TWA $\left(^{\circ}\right)$ & AWS (kn) & AWA $\left(^{\circ}\right)$ & $\mathrm{Mx}$ (N.m) \\
\hline 31.0 & 8.406 & -1.272 & -1.250 & 0.863 & 8.000 & 70.000 & 13.441 & 34.006 & -1.890 \\
\hline 31.1 & 8.405 & -1.260 & -1.250 & 0.863 & 8.000 & 70.000 & 13.441 & 34.009 & -2.480 \\
\hline 31.2 & 8.404 & -1.259 & -1.250 & 0.862 & 8.000 & 70.000 & 13.440 & 34.011 & -2.930 \\
\hline 31.3 & 8.404 & -1.272 & -1.250 & 0.862 & 8.000 & 70.000 & 13.439 & 34.013 & -3.180 \\
\hline 31.4 & 8.403 & -1.299 & -1.250 & 0.863 & 8.000 & 70.000 & 13.439 & 34.014 & -3.200 \\
\hline 31.5 & 8.403 & -1.338 & -1.250 & 0.863 & 8.000 & 70.000 & 13.438 & 34.015 & -2.980 \\
\hline 31.6 & 8.403 & -1.387 & -1.250 & 0.865 & 8.000 & 70.000 & 13.438 & 34.015 & -2.490 \\
\hline 31.7 & 8.403 & -1.443 & -1.250 & 0.866 & 8.000 & 70.000 & 13.439 & 34.014 & -1.760 \\
\hline 31.8 & 8.404 & -1.503 & -1.250 & 0.868 & 8.000 & 70.000 & 13.439 & 34.012 & -0.830 \\
\hline 31.9 & 8.405 & -1.562 & -1.250 & 0.870 & 8.000 & 70.000 & 13.440 & 34.009 & 0.250 \\
\hline 32.0 & 8.407 & -1.615 & -1.250 & 0.872 & 8.000 & 70.000 & 13.442 & 34.006 & 1.410 \\
\hline 32.1 & 8.408 & -1.658 & -1.250 & 0.874 & 8.000 & 70.000 & 13.443 & 34.002 & 2.550 \\
\hline 32.2 & 8.410 & -1.686 & -1.250 & 0.875 & 8.000 & 70.000 & 13.444 & 33.998 & 3.580 \\
\hline 32.3 & 8.412 & -1.695 & -1.250 & 0.876 & 8.000 & 70.000 & 13.446 & 33.994 & 4.410 \\
\hline 32.4 & 8.413 & -1.683 & -1.250 & 0.876 & 8.000 & 70.000 & 13.447 & 33.990 & 4.960 \\
\hline 32.5 & 8.414 & -1.650 & -1.250 & 0.876 & 8.000 & 70.000 & 13.448 & 33.988 & 5.150 \\
\hline 32.6 & 8.415 & -1.597 & -1.250 & 0.875 & 8.000 & 70.000 & 13.448 & 33.986 & 4.950 \\
\hline 32.7 & 8.415 & -1.525 & -1.250 & 0.873 & 8.000 & 70.000 & 13.448 & 33.986 & 4.330 \\
\hline 32.8 & 8.414 & -1.439 & -1.250 & 0.871 & 8.000 & 70.000 & 13.448 & 33.987 & 3.320 \\
\hline
\end{tabular}


Table B.2 - Continued from previous page

\begin{tabular}{|c|c|c|c|c|c|c|c|c|c|}
\hline Time $(\mathrm{s})$ & $\mathrm{BSP}(\mathrm{kn})$ & Heel $\left(^{\circ}\right)$ & Crew Y Pos. $(\mathrm{m})$ & Flat & TWS (kn) & TWA $\left(^{\circ}\right)$ & AWS (kn) & AWA $\left(^{\circ}\right)$ & $\mathrm{Mx}$ (N.m) \\
\hline 32.9 & 8.413 & -1.345 & -1.250 & 0.868 & 8.000 & 70.000 & 13.447 & 33.990 & 1.970 \\
\hline 33.0 & 8.412 & -1.250 & -1.250 & 0.865 & 8.000 & 70.000 & 13.446 & 33.993 & 0.360 \\
\hline 33.1 & 8.410 & -1.161 & -1.250 & 0.862 & 8.000 & 70.000 & 13.444 & 33.998 & -1.420 \\
\hline 33.2 & 8.407 & -1.085 & -1.250 & 0.859 & 8.000 & 70.000 & 13.442 & 34.004 & -3.220 \\
\hline 33.3 & 8.405 & -1.029 & -1.250 & 0.857 & 8.000 & 70.000 & 13.440 & 34.011 & -4.930 \\
\hline 33.4 & 8.402 & -1.001 & -1.250 & 0.855 & 8.000 & 70.000 & 13.438 & 34.017 & -6.400 \\
\hline 33.5 & 8.399 & -1.003 & -1.250 & 0.854 & 8.000 & 70.000 & 13.436 & 34.023 & -7.490 \\
\hline 33.6 & 8.397 & -1.039 & -1.250 & 0.854 & 8.000 & 70.000 & 13.434 & 34.028 & -8.090 \\
\hline 33.7 & 8.396 & -1.109 & -1.250 & 0.855 & 8.000 & 70.000 & 13.433 & 34.031 & -8.110 \\
\hline 33.8 & 8.395 & -1.210 & -1.250 & 0.857 & 8.000 & 70.000 & 13.432 & 34.033 & -7.490 \\
\hline 33.9 & 8.396 & -1.337 & -1.250 & 0.861 & 8.000 & 70.000 & 13.432 & 34.032 & -6.220 \\
\hline 34.0 & 8.397 & -1.482 & -1.250 & 0.865 & 8.000 & 70.000 & 13.433 & 34.029 & -4.320 \\
\hline 34.1 & 8.399 & -1.635 & -1.250 & 0.869 & 8.000 & 70.000 & 13.435 & 34.024 & -1.900 \\
\hline 34.2 & 8.402 & -1.784 & -1.250 & 0.874 & 8.000 & 70.000 & 13.438 & 34.017 & 0.900 \\
\hline 34.3 & 8.406 & -1.918 & -1.250 & 0.879 & 8.000 & 70.000 & 13.441 & 34.008 & 3.870 \\
\hline 34.4 & 8.410 & -2.023 & -1.250 & 0.883 & 8.000 & 70.000 & 13.444 & 33.998 & 6.790 \\
\hline 34.5 & 8.414 & -2.089 & -1.250 & 0.887 & 8.000 & 70.000 & 13.448 & 33.988 & 9.410 \\
\hline 34.6 & 8.418 & -2.107 & -1.250 & 0.889 & 8.000 & 70.000 & 13.451 & 33.978 & 11.460 \\
\hline 34.7 & 8.422 & -2.071 & -1.250 & 0.889 & 8.000 & 70.000 & 13.454 & 33.970 & 12.750 \\
\hline \multicolumn{10}{|c|}{ Continue on next page } \\
\hline
\end{tabular}


Table B.2 - Continued from previous page

\begin{tabular}{|c|c|c|c|c|c|c|c|c|c|}
\hline Time $(\mathrm{s})$ & BSP $(\mathrm{kn})$ & Heel $\left(^{\circ}\right)$ & Crew Y Pos. $(\mathrm{m})$ & Flat & TWS $(\mathrm{kn})$ & TWA $\left(^{\circ}\right)$ & AWS $(\mathrm{kn})$ & AWA $\left(^{\circ}\right)$ & Mx $($ N.m) \\
\hline 34.8 & 8.424 & -1.981 & -1.250 & 0.888 & 8.000 & 70.000 & 13.456 & 33.964 & 13.100 \\
\hline 34.9 & 8.426 & -1.839 & -1.250 & 0.885 & 8.000 & 70.000 & 13.458 & 33.960 & 12.430 \\
\hline 35.0 & 8.426 & -1.652 & -1.250 & 0.881 & 8.000 & 70.000 & 13.458 & 33.960 & 10.710 \\
\hline
\end{tabular}




\section{B.2 Time History Data of the Simulated Foiling Moth Transition Run}

Table B.3 shows the time history of some of the calculated variables during the Moth foiler time-domain simulated transition from non-foiling to foiling presented in section in section 3.7.3.

Tables B.4 and B.5 show the associated kinematic cues for the Stewart Platform of the first four seconds of the simulated transition, calculated using the Classical Washout and the Adaptive Washout respectively, and the platform kinematic algorithms described in Chapter 4. The platform geometry used is similar to the geometry of the SIROCCO hexapod from Symetrie [168]. 
Table B.3: Time History of the Simulated Foiling Moth Transition Run

\begin{tabular}{|c|c|c|c|c|c|c|c|c|c|c|}
\hline Time (s) & TWS (kn) & AWS (kn) & AWA $\left(^{\circ}\right)$ & $\mathrm{BSP}(\mathrm{kn})$ & $\operatorname{Pitch}\left(^{\circ}\right)$ & Yaw $\left(^{\circ}\right)$ & Sink $(\mathrm{m})$ & Crew Y Pos. $(\mathrm{m})$ & Flat & Rud. Ang. $\left({ }^{\circ}\right)$ \\
\hline 0 & 6.700 & 8.296 & 53.863 & 4.892 & -2.793 & 0.879 & 0.100 & -0.368 & 1.000 & -0.486 \\
\hline 1 & 7.349 & 8.849 & 56.147 & 4.929 & -2.748 & 0.946 & 0.099 & -0.407 & 1.000 & -0.441 \\
\hline 2 & 7.999 & 9.452 & 57.808 & 5.036 & -2.758 & 1.024 & 0.098 & -0.458 & 1.000 & -0.432 \\
\hline 3 & 8.499 & 9.962 & 58.553 & 5.197 & -2.827 & 1.066 & 0.096 & -0.508 & 1.000 & -0.430 \\
\hline 4 & 8.999 & 10.497 & 59.012 & 5.405 & -2.931 & 1.090 & 0.093 & -0.566 & 1.000 & -0.429 \\
\hline 5 & 9.499 & 11.056 & 59.225 & 5.657 & -3.106 & 1.102 & 0.091 & -0.634 & 1.000 & -0.434 \\
\hline 6 & 9.999 & 11.642 & 59.194 & 5.962 & -3.376 & 1.109 & 0.089 & -0.712 & 1.000 & -0.455 \\
\hline 7 & 9.999 & 11.812 & 57.831 & 6.289 & -3.508 & 1.042 & 0.085 & -0.759 & 1.000 & -0.458 \\
\hline 8 & 9.999 & 11.984 & 56.547 & 6.606 & -3.423 & 0.967 & 0.080 & -0.804 & 1.000 & -0.433 \\
\hline 9 & 9.999 & 12.158 & 55.329 & 6.916 & -3.326 & 0.903 & 0.074 & -0.849 & 1.000 & -0.414 \\
\hline 10 & 9.999 & 12.330 & 54.192 & 7.214 & -3.228 & 0.848 & 0.068 & -0.893 & 1.000 & -0.396 \\
\hline 11 & 9.999 & 12.497 & 53.144 & 7.496 & -3.141 & 0.801 & 0.061 & -0.936 & 1.000 & -0.381 \\
\hline 12 & 9.999 & 12.659 & 52.175 & 7.763 & -3.055 & 0.761 & 0.054 & -0.978 & 1.000 & -0.367 \\
\hline 13 & 9.999 & 12.819 & 51.264 & 8.021 & -2.961 & 0.725 & 0.045 & -1.020 & 1.000 & -0.351 \\
\hline 14 & 9.999 & 12.982 & 50.376 & 8.279 & -2.863 & 0.691 & 0.034 & -1.062 & 1.000 & -0.334 \\
\hline 15 & 9.999 & 13.159 & 49.452 & 8.554 & -2.774 & 0.659 & 0.020 & -1.109 & 1.000 & -0.316 \\
\hline 16 & 9.999 & 13.370 & 48.404 & 8.876 & -2.721 & 0.626 & 0.003 & -1.165 & 1.000 & -0.303 \\
\hline 17 & 9.999 & 13.605 & 47.301 & 9.226 & -2.528 & 0.664 & -0.029 & -1.224 & 1.000 & -0.334 \\
\hline 18 & 9.999 & 13.788 & 46.483 & 9.494 & -1.582 & 0.709 & -0.135 & -1.250 & 0.973 & -0.270 \\
\hline & & & & & & & & & \multicolumn{2}{|c|}{ Continue on next page } \\
\hline
\end{tabular}


Table B.3 - Continued from previous page

\begin{tabular}{|c|c|c|c|c|c|c|c|c|c|c|}
\hline Time $(\mathrm{s})$ & TWS $(\mathrm{kn})$ & AWS $(\mathrm{kn})$ & AWA $\left(^{\circ}\right)$ & BSP $(\mathrm{kn})$ & Pitch $\left(^{\circ}\right)$ & Yaw $\left(^{\circ}\right)$ & Sink $(\mathrm{m})$ & Crew Y Pos. $(\mathrm{m})$ & Flat & Rud. Ang. $\left(^{\circ}\right)$ \\
\hline 19 & 9.999 & 13.945 & 45.808 & 9.721 & -0.896 & 0.890 & -0.277 & -1.250 & 0.937 & -0.303 \\
\hline 20 & 9.999 & 14.135 & 45.025 & 9.990 & -1.114 & 1.099 & -0.383 & -1.250 & 0.899 & -0.412 \\
\hline 21 & 9.999 & 14.366 & 44.108 & 10.315 & -1.780 & 1.192 & -0.429 & -1.250 & 0.859 & -0.504 \\
\hline 22 & 9.999 & 14.606 & 43.202 & 10.647 & -2.169 & 1.175 & -0.446 & -1.250 & 0.820 & -0.531 \\
\hline 23 & 9.999 & 14.822 & 42.424 & 10.941 & -2.167 & 1.164 & -0.466 & -1.250 & 0.789 & -0.536 \\
\hline 24 & 9.999 & 15.011 & 41.766 & 11.197 & -2.162 & 1.186 & -0.491 & -1.250 & 0.764 & -0.553 \\
\hline \multirow{8}{*}{5} & 9.999 & 15.184 & 41.187 & 11.427 & -2.254 & 1.203 & -0.509 & -1.250 & 0.743 & -0.571 \\
\hline 26 & 9.999 & 15.341 & 40.676 & 11.635 & -2.329 & 1.205 & -0.521 & -1.250 & 0.724 & -0.582 \\
\hline 27 & 9.999 & 15.481 & 40.232 & 11.819 & -2.365 & 1.206 & -0.532 & -1.250 & 0.708 & -0.588 \\
\hline 28 & 9.999 & 15.606 & 39.846 & 11.981 & -2.397 & 1.207 & -0.541 & -1.250 & 0.694 & -0.594 \\
\hline 29 & 9.999 & 15.716 & 39.511 & 12.125 & -2.428 & 1.206 & -0.548 & -1.250 & 0.683 & -0.599 \\
\hline 30 & 9.999 & 15.814 & 39.220 & 12.251 & -2.452 & 1.205 & -0.554 & -1.250 & 0.673 & -0.602 \\
\hline 31 & 9.999 & 15.900 & 38.967 & 12.362 & -2.472 & 1.203 & -0.560 & -1.250 & 0.664 & -0.605 \\
\hline 32 & 9.999 & 15.976 & 38.747 & 12.460 & -2.489 & 1.201 & -0.564 & -1.250 & 0.657 & -0.606 \\
\hline 33 & 9.999 & 16.043 & 38.555 & 12.546 & -2.504 & 1.199 & -0.568 & -1.250 & 0.651 & -0.608 \\
\hline 34 & 9.999 & 16.101 & 38.389 & 12.620 & -2.516 & 1.197 & -0.571 & -1.250 & 0.645 & -0.609 \\
\hline 35 & 9.999 & 16.153 & 38.245 & 12.686 & -2.527 & 1.195 & -0.573 & -1.250 & 0.641 & -0.610 \\
\hline
\end{tabular}


Table B.4: Kinematic cues of the first four seconds of the Foiling Moth Transition Run Using Classical Washout

\begin{tabular}{|c|c|c|c|c|c|c|c|c|c|c|c|c|}
\hline \multirow{2}{*}{ Time (s) } & \multicolumn{2}{|c|}{ Leg 1} & \multicolumn{2}{|c|}{ Leg 2} & \multicolumn{2}{|c|}{ Leg 3} & \multicolumn{2}{|c|}{ Leg 4} & \multicolumn{2}{|c|}{ Leg 5} & \multicolumn{2}{|c|}{ Leg 6} \\
\hline & Ext. (m) & Acc. $\left(m / s^{2}\right)$ & Ext. (m) & Acc. $\left(m / s^{2}\right)$ & Ext. (m) & Acc. $\left(m / s^{2}\right)$ & Ext. (m) & Acc. $\left(m / s^{2}\right)$ & Ext. (m) & Acc. $\left(m / s^{2}\right)$ & Ext. (m) & Acc. $\left(m / s^{2}\right)$ \\
\hline 0.1 & 2.931 & 1.957 & 2.931 & 1.958 & 2.931 & 1.959 & 2.931 & 1.959 & 2.931 & 1.958 & 2.931 & 1.957 \\
\hline 0.2 & 2.973 & 1.396 & 2.973 & 1.399 & 2.973 & 1.403 & 2.973 & 1.404 & 2.973 & 1.401 & 2.973 & 1.397 \\
\hline 0.3 & 3.016 & 0.151 & 3.016 & 0.154 & 3.016 & 0.160 & 3.016 & 0.163 & 3.016 & 0.159 & 3.016 & 0.153 \\
\hline 0.4 & 3.055 & -0.419 & 3.055 & -0.416 & 3.056 & -0.410 & 3.056 & -0.408 & 3.056 & -0.412 & 3.055 & -0.417 \\
\hline 0.5 & 3.088 & $\begin{array}{l}-0.625 \\
\end{array}$ & 3.089 & $\begin{array}{l}-0.621 \\
\end{array}$ & 3.089 & -0.617 & 3.089 & $\begin{array}{l}-0.617 \\
\end{array}$ & 3.089 & $\begin{array}{l}-0.621 \\
\end{array}$ & 3.088 & $\begin{array}{l}-0.625 \\
\end{array}$ \\
\hline 0.6 & 3.115 & -0.647 & 3.115 & -0.644 & 3.116 & -0.641 & 3.116 & -0.642 & 3.116 & -0.646 & 3.115 & -0.648 \\
\hline 0.7 & 3.135 & -0.584 & 3.136 & -0.582 & 3.137 & -0.581 & 3.137 & -0.582 & 3.137 & -0.585 & 3.136 & -0.586 \\
\hline 0.8 & 3.151 & $\begin{array}{l}-0.492 \\
\end{array}$ & 3.152 & -0.490 & 3.153 & -0.490 & 3.154 & -0.491 & 3.153 & -0.493 & 3.151 & -0.493 \\
\hline 0.9 & 3.163 & -0.396 & 3.164 & -0.395 & 3.166 & -0.395 & 3.166 & -0.396 & 3.165 & -0.397 & 3.163 & -0.396 \\
\hline 1.0 & 3.172 & -0.309 & 3.173 & -0.309 & 3.175 & -0.310 & 3.175 & -0.310 & 3.174 & -0.310 & 3.172 & -0.309 \\
\hline 1.1 & 3.178 & -0.236 & 3.180 & -0.237 & 3.182 & -0.237 & 3.182 & -0.238 & 3.180 & -0.237 & 3.178 & -0.236 \\
\hline 1.2 & 3.182 & $\begin{array}{c}-0.177 \\
\end{array}$ & 3.184 & -0.178 & 3.187 & -0.179 & 3.187 & $\begin{array}{c}-0.179 \\
\end{array}$ & 3.185 & $\begin{array}{l}-0.179 \\
\end{array}$ & 3.183 & $\begin{array}{c}-0.177 \\
\end{array}$ \\
\hline 1.3 & 3.186 & -0.131 & 3.188 & -0.132 & 3.190 & -0.133 & 3.191 & -0.133 & 3.188 & -0.133 & 3.186 & -0.131 \\
\hline 1.4 & 3.188 & $\begin{array}{l}-0.096 \\
\end{array}$ & 3.190 & $\begin{array}{ll}-0.097 \\
\end{array}$ & 3.193 & $\begin{array}{l}-0.098 \\
\end{array}$ & 3.193 & $\begin{array}{l}-0.098 \\
\end{array}$ & 3.191 & $\begin{array}{l}-0.097 \\
\end{array}$ & 3.188 & $\begin{array}{l}-0.096 \\
\end{array}$ \\
\hline 1.5 & 3.190 & -0.070 & 3.192 & -0.071 & 3.195 & -0.072 & 3.195 & -0.072 & 3.193 & -0.071 & 3.190 & -0.070 \\
\hline 1.6 & 3.191 & $\begin{array}{l}-0.050 \\
\end{array}$ & 3.193 & -0.051 & 3.196 & -0.052 & 3.197 & -0.052 & 3.194 & -0.051 & 3.191 & $\begin{array}{l}-0.050 \\
\end{array}$ \\
\hline 1.7 & 3.191 & -0.036 & 3.194 & -0.037 & 3.197 & -0.038 & 3.198 & -0.038 & 3.195 & -0.037 & 3.192 & -0.036 \\
\hline 1.8 & 3.192 & -0.025 & 3.195 & -0.026 & 3.198 & -0.027 & 3.199 & -0.027 & 3.195 & -0.027 & 3.192 & $\begin{array}{l}-0.026 \\
\end{array}$ \\
\hline 1.9 & 3.192 & -0.018 & 3.195 & -0.019 & 3.199 & -0.019 & 3.199 & -0.020 & 3.196 & $\begin{array}{c}-0.019 \\
\end{array}$ & 3.193 & $\begin{array}{c}-0.018 \\
\end{array}$ \\
\hline 2.0 & 3.192 & -0.013 & 3.195 & -0.013 & 3.199 & -0.014 & 3.200 & -0.014 & 3.196 & -0.013 & 3.193 & -0.013 \\
\hline 2.1 & 3.193 & -0.009 & 3.196 & -0.009 & 3.200 & -0.010 & 3.200 & -0.010 & 3.196 & -0.010 & 3.193 & -0.009 \\
\hline 2.2 & 3.193 & -0.006 & 3.196 & -0.006 & 3.200 & -0.007 & 3.200 & -0.007 & 3.196 & -0.007 & 3.193 & -0.006 \\
\hline 2.3 & 3.193 & -0.004 & 3.196 & -0.005 & 3.200 & -0.005 & 3.201 & -0.005 & 3.197 & -0.005 & 3.193 & -0.004 \\
\hline 2.4 & 3.192 & $\begin{array}{l}-0.003 \\
\end{array}$ & 3.196 & -0.003 & 3.200 & -0.004 & 3.201 & $\begin{array}{l}-0.004 \\
\end{array}$ & 3.197 & $\begin{array}{l}-0.003 \\
\end{array}$ & 3.193 & $\begin{array}{l}-0.003 \\
\end{array}$ \\
\hline 2.5 & 3.192 & $\begin{array}{l}-0.002 \\
\end{array}$ & 3.196 & -0.002 & 3.201 & -0.002 & 3.201 & -0.003 & 3.197 & $\begin{array}{l}-0.002 \\
\end{array}$ & 3.193 & $\begin{array}{l}-0.002 \\
\end{array}$ \\
\hline 2.6 & 3.192 & -0.001 & 3.196 & -0.002 & 3.201 & -0.002 & 3.201 & $\begin{array}{l}-0.002 \\
\end{array}$ & 3.197 & $\begin{array}{l}-0.002 \\
\end{array}$ & 3.193 & $\begin{array}{l}-0.001 \\
\end{array}$ \\
\hline 2.7 & 3.192 & -0.001 & 3.196 & $\begin{array}{l}-0.001 \\
\end{array}$ & 3.201 & -0.001 & 3.201 & -0.001 & 3.197 & -0.001 & 3.192 & -0.001 \\
\hline 2.8 & 3.192 & $\begin{array}{c}-0.001 \\
\end{array}$ & 3.196 & $\begin{array}{c}-0.001 \\
\end{array}$ & 3.201 & -0.001 & 3.201 & $\begin{array}{c}-0.001 \\
\end{array}$ & 3.197 & $\begin{array}{c}-0.001 \\
\end{array}$ & 3.192 & $\begin{array}{c}-0.001 \\
\end{array}$ \\
\hline 2.9 & 3.192 & 0.000 & 3.196 & -0.001 & 3.201 & -0.001 & 3.202 & -0.001 & 3.197 & 0.000 & 3.192 & 0.000 \\
\hline 3.0 & 3.192 & 0.000 & 3.196 & 0.000 & 3.201 & -0.001 & 3.202 & 0.000 & 3.197 & 0.000 & 3.192 & 0.000 \\
\hline 3.1 & 3.192 & 0.000 & 3.196 & 0.000 & 3.202 & -0.001 & 3.202 & 0.000 & 3.197 & 0.000 & 3.192 & 0.000 \\
\hline 3.2 & 3.192 & 0.000 & 3.196 & 0.000 & 3.202 & -0.001 & 3.202 & 0.000 & 3.197 & 0.000 & 3.192 & 0.000 \\
\hline 3.3 & 3.192 & 0.000 & 3.196 & 0.000 & 3.202 & -0.001 & 3.202 & 0.000 & 3.197 & 0.000 & 3.192 & 0.000 \\
\hline
\end{tabular}


Table B.4 - Continued from previous page

\begin{tabular}{|c|c|c|c|c|c|c|c|c|c|c|c|c|}
\hline \multirow{2}{*}{ Time (s) } & \multicolumn{2}{|c|}{ Leg 1} & \multicolumn{2}{|c|}{ Leg 2} & \multicolumn{2}{|c|}{ Leg 3} & \multicolumn{2}{|c|}{ Leg 4} & \multicolumn{2}{|c|}{ Leg 5} & \multicolumn{2}{|c|}{ Leg 6} \\
\hline & Ext. (m) & Acc. $\left(m / s^{2}\right)$ & Ext. (m) & Acc. $\left(m / s^{2}\right)$ & Ext. (m) & Acc. $\left(m / s^{2}\right)$ & Ext. (m) & Acc. $\left(m / s^{2}\right)$ & Ext. (m) & Acc. $\left(m / s^{2}\right)$ & Ext. (m) & Acc. $\left(\mathrm{m} / \mathrm{s}^{2}\right)$ \\
\hline 3.4 & 3.192 & 0.000 & 3.196 & 0.000 & 3.202 & 0.000 & 3.202 & 0.000 & 3.197 & 0.000 & 3.192 & 0.000 \\
\hline 3.5 & 3.191 & 0.000 & 3.196 & 0.000 & 3.202 & 0.000 & 3.202 & 0.000 & 3.197 & 0.000 & 3.192 & 0.000 \\
\hline 3.6 & 3.191 & 0.000 & 3.196 & 0.000 & 3.202 & 0.000 & 3.202 & 0.000 & 3.197 & 0.000 & 3.192 & 0.000 \\
\hline 3.7 & 3.191 & 0.000 & 3.196 & 0.000 & 3.202 & 0.000 & 3.202 & 0.000 & 3.197 & 0.000 & 3.192 & 0.000 \\
\hline 3.8 & 3.191 & 0.000 & 3.196 & 0.000 & 3.202 & 0.000 & 3.203 & 0.000 & 3.197 & 0.000 & 3.191 & 0.000 \\
\hline 3.9 & 3.191 & 0.000 & 3.196 & 0.000 & 3.202 & 0.000 & 3.203 & 0.000 & 3.196 & 0.000 & 3.191 & 0.000 \\
\hline 4.0 & 3.191 & 0.000 & 3.196 & 0.000 & 3.203 & 0.000 & 3.203 & 0.000 & 3.196 & 0.000 & 3.191 & 0.000 \\
\hline
\end{tabular}


Table B.5: Kinematic cues of the first four seconds of the Foiling Moth Transition Run Using Adaptive Washout

\begin{tabular}{|c|c|c|c|c|c|c|c|c|c|c|c|c|}
\hline \multirow{2}{*}{ Time (s) } & \multicolumn{2}{|c|}{ Leg 1} & \multicolumn{2}{|c|}{ Leg 2} & \multicolumn{2}{|c|}{ Leg 3} & \multicolumn{2}{|c|}{ Leg 4} & \multicolumn{2}{|c|}{ Leg 5} & \multicolumn{2}{|c|}{ Leg 6} \\
\hline & Ext. (m) & Acc. $\left(m / s^{2}\right)$ & Ext. (m) & Acc. $\left(m / s^{2}\right)$ & Ext. (m) & Acc. $\left(m / s^{2}\right)$ & Ext. (m) & Acc. $\left(m / s^{2}\right)$ & Ext. (m) & Acc. $\left(m / s^{2}\right)$ & Ext. (m) & Acc. $\left(m / s^{2}\right)$ \\
\hline 0.1 & 2.894 & 0.000 & 2.894 & 0.000 & 2.894 & 0.000 & 2.894 & 0.000 & 2.894 & 0.000 & 2.894 & 0.000 \\
\hline 0.2 & 2.894 & -0.004 & 2.894 & 0.000 & 2.894 & 0.003 & 2.894 & 0.003 & 2.894 & 0.000 & 2.894 & -0.003 \\
\hline 0.3 & 2.943 & 4.831 & 2.943 & 4.835 & 2.943 & 4.838 & 2.943 & 4.838 & 2.943 & 4.835 & 2.943 & 4.831 \\
\hline 0.4 & 3.003 & 1.159 & 3.003 & 1.163 & 3.003 & 1.168 & 3.003 & 1.168 & 3.003 & 1.164 & 3.003 & 1.159 \\
\hline 0.5 & 3.062 & $\begin{array}{l}-0.061 \\
\end{array}$ & 3.062 & -0.058 & 3.062 & -0.054 & 3.062 & $\begin{array}{l}-0.054 \\
\end{array}$ & 3.062 & $\begin{array}{l}-0.057 \\
\end{array}$ & 3.062 & $\begin{array}{l}-0.061 \\
\end{array}$ \\
\hline 0.6 & 3.110 & -1.052 & 3.111 & -1.049 & 3.111 & -1.045 & 3.111 & -1.045 & 3.111 & -1.049 & 3.111 & -1.051 \\
\hline 0.7 & 3.135 & -2.393 & 3.136 & -2.391 & 3.136 & -2.388 & 3.136 & -2.388 & 3.136 & -2.391 & 3.135 & $\begin{array}{l}-2.392 \\
\end{array}$ \\
\hline 0.8 & 3.130 & -3.019 & 3.131 & -3.018 & 3.131 & -3.015 & 3.131 & -3.015 & 3.131 & -3.018 & 3.130 & -3.019 \\
\hline 0.9 & 3.106 & -1.817 & 3.107 & -1.817 & 3.108 & -1.814 & 3.108 & -1.814 & 3.107 & -1.817 & 3.106 & -1.817 \\
\hline 1.0 & 3.079 & -0.415 & 3.080 & -0.415 & 3.081 & -0.413 & 3.081 & -0.413 & 3.080 & -0.415 & 3.079 & -0.415 \\
\hline 1.1 & 3.052 & 0.049 & 3.053 & 0.049 & 3.054 & 0.051 & 3.054 & 0.051 & 3.053 & 0.049 & 3.052 & 0.049 \\
\hline 1.2 & 3.026 & 0.191 & 3.028 & 0.191 & 3.030 & 0.192 & 3.030 & 0.192 & 3.028 & 0.191 & 3.026 & 0.191 \\
\hline 1.3 & 3.003 & 0.224 & 3.005 & 0.223 & 3.007 & 0.224 & 3.007 & 0.224 & 3.005 & 0.223 & 3.003 & 0.224 \\
\hline 1.4 & 2.982 & 0.220 & 2.984 & 0.219 & 2.987 & 0.220 & 2.987 & 0.220 & 2.984 & 0.219 & 2.983 & 0.220 \\
\hline 1.5 & 2.964 & 0.205 & 2.965 & 0.204 & 2.968 & 0.205 & 2.968 & 0.205 & 2.965 & 0.204 & 2.964 & 0.205 \\
\hline 1.6 & 2.947 & 0.187 & 2.948 & 0.186 & 2.952 & 0.187 & 2.952 & 0.187 & 2.949 & 0.186 & 2.947 & 0.187 \\
\hline 1.7 & 2.931 & 0.170 & 2.933 & 0.169 & 2.937 & 0.170 & 2.937 & 0.170 & 2.934 & 0.169 & 2.932 & 0.170 \\
\hline 1.8 & 2.918 & 0.203 & 2.920 & 0.202 & 2.924 & 0.203 & 2.924 & 0.203 & 2.920 & 0.202 & 2.918 & 0.203 \\
\hline 1.9 & 2.908 & 0.296 & 2.910 & 0.295 & 2.915 & 0.296 & 2.914 & 0.296 & 2.910 & 0.295 & 2.908 & 0.296 \\
\hline 2.0 & 2.902 & 0.385 & 2.904 & 0.385 & 2.909 & 0.385 & 2.909 & 0.385 & 2.904 & 0.385 & 2.902 & 0.385 \\
\hline 2.1 & 2.900 & 0.443 & 2.902 & 0.443 & 2.907 & 0.443 & 2.907 & 0.443 & 2.902 & 0.443 & 2.900 & 0.443 \\
\hline 2.2 & 2.902 & 0.462 & 2.905 & 0.462 & 2.910 & 0.462 & 2.910 & 0.463 & 2.905 & 0.462 & 2.903 & 0.462 \\
\hline 2.3 & 2.909 & 0.442 & 2.912 & 0.442 & 2.918 & 0.443 & 2.918 & 0.443 & 2.912 & 0.442 & 2.910 & 0.442 \\
\hline 2.4 & 2.920 & 0.388 & 2.923 & 0.388 & 2.929 & 0.388 & 2.929 & 0.388 & 2.923 & 0.388 & 2.921 & 0.388 \\
\hline 2.5 & 2.934 & 0.306 & 2.937 & 0.307 & 2.944 & 0.307 & 2.944 & 0.307 & 2.937 & 0.306 & 2.935 & 0.306 \\
\hline 2.6 & 2.951 & 0.206 & 2.953 & 0.206 & 2.960 & 0.206 & 2.960 & 0.206 & 2.954 & 0.206 & 2.951 & 0.205 \\
\hline 2.7 & 2.968 & 0.095 & 2.970 & 0.096 & 2.978 & 0.096 & 2.978 & 0.096 & 2.971 & 0.096 & 2.968 & 0.095 \\
\hline 2.8 & 2.984 & $\begin{array}{l}-0.016 \\
\end{array}$ & 2.987 & $\begin{array}{l}-0.016 \\
\end{array}$ & 2.995 & $\begin{array}{l}-0.015 \\
\end{array}$ & 2.995 & $\begin{array}{l}-0.015 \\
\end{array}$ & 2.988 & $\begin{array}{l}-0.016 \\
\end{array}$ & 2.985 & $\begin{array}{l}-0.016 \\
\end{array}$ \\
\hline 2.9 & 3.000 & -0.119 & 3.003 & -0.119 & 3.011 & -0.119 & 3.011 & -0.119 & 3.003 & -0.119 & 3.001 & -0.119 \\
\hline 3.0 & 3.014 & -0.208 & 3.017 & -0.208 & 3.026 & -0.207 & 3.026 & -0.207 & 3.017 & -0.208 & 3.014 & -0.208 \\
\hline 3.1 & 3.025 & -0.276 & 3.028 & -0.276 & 3.037 & -0.276 & 3.037 & -0.276 & 3.028 & -0.276 & 3.025 & -0.276 \\
\hline 3.2 & 3.032 & -0.319 & 3.036 & -0.320 & 3.045 & -0.320 & 3.045 & -0.320 & 3.036 & -0.320 & 3.033 & -0.319 \\
\hline 3.3 & 3.037 & -0.337 & 3.040 & -0.338 & 3.050 & -0.338 & 3.050 & -0.338 & 3.040 & -0.338 & 3.037 & -0.337 \\
\hline
\end{tabular}


Table B.5 - Continued from previous page

\begin{tabular}{|c|c|c|c|c|c|c|c|c|c|c|c|c|}
\hline \multirow{2}{*}{ Time $(\mathrm{s})$} & \multicolumn{2}{|c|}{ Leg 1} & \multicolumn{2}{|c|}{ Leg 2} & \multicolumn{2}{|c|}{ Leg 3} & \multicolumn{2}{|c|}{ Leg 4} & \multicolumn{2}{|c|}{ Leg 5} & \multicolumn{2}{|c|}{ Leg 6} \\
\hline & Ext. (m) & Acc. $\left(m / s^{2}\right)$ & Ext. (m) & Acc. $\left(m / s^{2}\right)$ & Ext. (m) & Acc. $\left(m / s^{2}\right)$ & Ext. (m) & Acc. $\left(m / s^{2}\right)$ & Ext. (m) & Acc. $\left(m / s^{2}\right)$ & Ext. (m) & Acc. $\left(m / s^{2}\right)$ \\
\hline 3.4 & 3.038 & -0.330 & 3.041 & -0.331 & 3.051 & -0.331 & 3.051 & -0.331 & 3.041 & -0.331 & 3.038 & -0.330 \\
\hline 3.5 & 3.036 & -0.300 & 3.039 & -0.301 & 3.050 & -0.301 & 3.050 & -0.301 & 3.039 & -0.301 & 3.036 & -0.300 \\
\hline 3.6 & 3.031 & -0.251 & 3.035 & -0.252 & 3.046 & -0.252 & 3.046 & -0.252 & 3.035 & -0.252 & 3.032 & -0.251 \\
\hline 3.7 & 3.025 & -0.188 & 3.028 & -0.189 & 3.040 & -0.189 & 3.040 & -0.189 & 3.029 & -0.189 & 3.025 & -0.188 \\
\hline 3.8 & 3.017 & $\begin{array}{l}-0.116 \\
\end{array}$ & 3.021 & $\begin{array}{l}-0.117 \\
\end{array}$ & 3.033 & -0.118 & 3.033 & -0.118 & 3.021 & $\begin{array}{l}-0.117 \\
\end{array}$ & 3.018 & $\begin{array}{l}-0.116 \\
\end{array}$ \\
\hline 3.9 & 3.009 & -0.043 & 3.013 & -0.043 & 3.025 & -0.044 & 3.025 & -0.044 & 3.013 & -0.043 & 3.010 & -0.043 \\
\hline 4.0 & 3.002 & 0.028 & 3.005 & 0.027 & 3.018 & 0.027 & 3.018 & 0.027 & 3.006 & 0.027 & 3.002 & 0.028 \\
\hline
\end{tabular}




\section{Appendix C}

\section{Multi-Threading Tests Time Results}

The following table shows the results of nine tests carried out to measure the timesaving improvement when using multi-threading calculating with the Moth foiler models. The forces and moments models were divided into blocks. For a complete description of the tests carried and the block division please refer to section 3.6.

Table C.1: Multi-threading tests time results using Moth Foiler models

\begin{tabular}{|c|c|c|c|}
\hline Test Number & Iteration Values Initialization & Block Number & Time (s) \\
\hline & & 2 & 0.4907864 \\
\hline & & 3 & 0.0107857 \\
\hline & & 4 & 0.7292886 \\
\hline & & 5 & 0.0038587 \\
\hline & & 6 & 0.0098918 \\
\hline & & 7 & 0.0011640 \\
\hline & & 8 & 0.0037806 \\
\hline & & All & 1.2727258 \\
\hline & \multirow{7}{*}{ Yes } & 1 & 0.0568333 \\
\hline & & 2 & 0.5727148 \\
\hline & & 3 & 0.0097887 \\
\hline & & 4 & 0.5727770 \\
\hline & & 5 & 0.0011242 \\
\hline & & 6 & 0.0027489 \\
\hline & & 7 & 0.0003613 \\
\hline
\end{tabular}


Table C.1 - Continued from previous page

\begin{tabular}{|l|c|c|c|}
\hline Test Number & Iteration Values Initialization & Block Number & Time $(\mathrm{s})$ \\
\hline & & 8 & 0.0003201 \\
\cline { 3 - 4 } & & All & 1.2166683 \\
\hline \multicolumn{2}{|c}{} \\
\cline { 3 - 4 }
\end{tabular}


Table C.1 - Continued from previous page

\begin{tabular}{|c|c|c|c|}
\hline Test Number & Iteration Values Initialization & Block Number & Time (s) \\
\hline $1^{*}$ & No & All & 1.0302808 \\
\hline \multirow{18}{*}{2} & \multirow{9}{*}{ No } & 1 & 0.0246999 \\
\hline & & 2 & 1.7335569 \\
\hline & & 3 & 0.0643968 \\
\hline & & 4 & 1.6961903 \\
\hline & & 5 & 0.0158485 \\
\hline & & 6 & 0.0369715 \\
\hline & & 7 & 0.0036877 \\
\hline & & 8 & 0.0034380 \\
\hline & & All & 3.5787896 \\
\hline & \multirow{9}{*}{ Yes } & 1 & 0.1737755 \\
\hline & & 2 & 1.7488922 \\
\hline & & 3 & 0.0650333 \\
\hline & & 4 & 1.7729522 \\
\hline & & 5 & 0.0142658 \\
\hline & & 6 & 0.0304211 \\
\hline & & 7 & 0.0026785 \\
\hline & & 8 & 0.0047040 \\
\hline & & All & 3.8127226 \\
\hline \multirow{4}{*}{3} & \multirow{4}{*}{ No } & Task 1 & 1.6593321 \\
\hline & & Task 2 & 1.6008096 \\
\hline & & Task 3 & 0.0525195 \\
\hline & & All & 3.3126612 \\
\hline $3^{*}$ & No & All & 1.8242162 \\
\hline 4 & No & All & 1.0652782 \\
\hline \multicolumn{4}{|c|}{ Continue on next page } \\
\hline
\end{tabular}


Table C.1 - Continued from previous page

\begin{tabular}{|c|c|c|c|}
\hline Test Number & Iteration Values Initialization & Block Number & Time (s) \\
\hline \multirow{9}{*}{5} & \multirow{9}{*}{ No } & 1 & 0.0090601 \\
\hline & & 2 & 1.6625537 \\
\hline & & 3 & 1.5831202 \\
\hline & & 4 & 0.0327341 \\
\hline & & 5 & 0.0059248 \\
\hline & & 6 & 0.0148495 \\
\hline & & 7 & 0.0009524 \\
\hline & & 8 & 0.0011864 \\
\hline & & All & 3.3103812 \\
\hline \multirow{4}{*}{6} & \multirow{4}{*}{ No } & Task 1 & 1.6197502 \\
\hline & & Task 2 & 1.6102061 \\
\hline & & Task 3 & 0.0512762 \\
\hline & & All & 3.2812325 \\
\hline 7 & No & All & 3.3301326 \\
\hline
\end{tabular}




\section{Appendix D}

\section{Moth Foiler Models}

\section{D.1 Aerodynamic Models}

\section{D.1.1 Sails}

Modified Sail model presented in Bögle et al. [18]:

This model adapts the coefficient curves and calculation procedure presented in Heikki Hansen's work [72] with specifically calculated coefficients for a limited range of AWA of a Moth mainsail using the North Sails software "Flow". It has been adapted to include the last IMS VPP [33] de-powering scheme using the flat parameter and the associated twist function but deactivating the reef parameter (the Moth mainsail is not reefable). The model only contemplates the mainsail but not the windage of the boat. It can be considered a mixed model, adapting external data but using a specific calculation procedure and an analytic de-powering scheme.

Aerodynamic model based on the TFWT experimental data:

This model is a pure interpolated model. It was generated during an experimental campaign held in the TFWT of the University of Auckland. A one-third scale model of the Moth was used for testing, including a scaled model of the crew (figure D.1). The boat trimming parameters can be remotely changed during testing. The data collected consists of the forces and moments measured by a six-degrees of freedom force balance. The experimental data matrix parameters are related to the boat position and wind characteristics:

- Boat foiling height (2 tested, 0.42 and 0.60 meters).

- Boat heel (5 tested ranging from -20 to 5 degrees). 


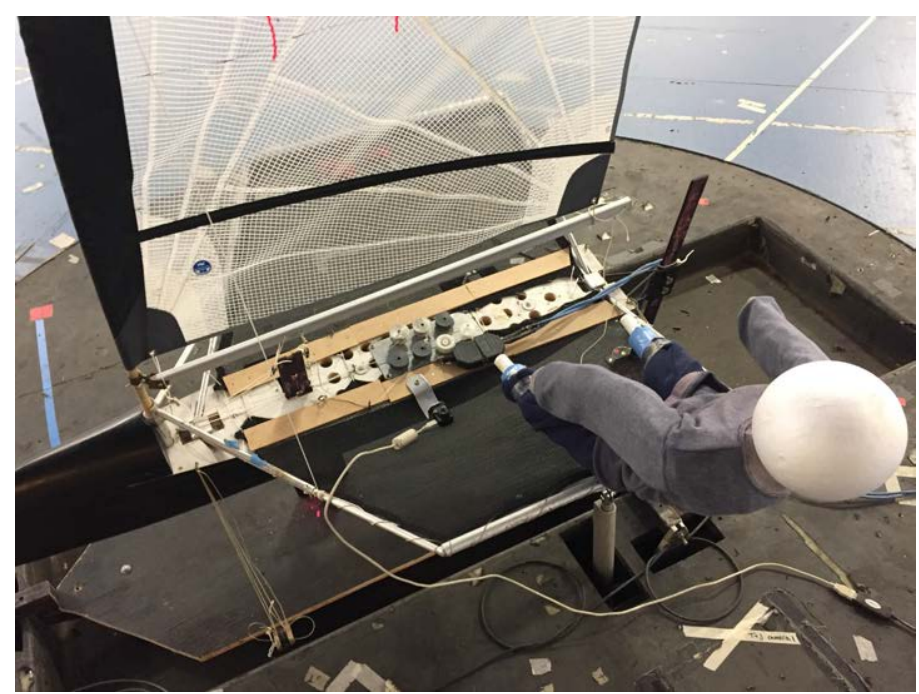

Figure D.1: Setup used in the TWFT for Moth aerodynamic model measurements

- Boat pitch (3 tested ranging from -4 to 4 degrees).

- AWA (8 tested ranging from 15 to 50 degrees).

- No vanes and two twisted flow distributions using vanes.

The initial idea was to cover all the parametric space but the resultant matrix was excessively large. The solution was to test the pitch parameter and the twisted flow distributions using a boat base position (0.42 meters of foiling height and -10 degrees of heel) for the complete AWA range. The effects on the pitch were measured to determine how it affects the force and moment coefficients. From the VPP perspective, the aerodynamic coefficients are determined for the principal matrix parameters (height, heel and AWA) using an interpolation scheme, and then they are affected by the pitch model presented previously. All the measurements were made for the best sail trim, maximizing the sail driving force in each measurement run. The model also includes a depowering scheme based on one trimming parameter (to reduce the number of data matrix parameters) using the main sheet to over-trim and under-trim the mainsail to change the heeling moment measured in best sail trim conditions (targeting a certain percentage increase or reduction of that value). The different vanes configurations were used to check how the twisted flow distribution affects the coefficients when sailing at low AWAs in comparison with the straight flow configuration (no vanes). The results obtained confirmed that straight flow measurements are close enough to twisted flow data and can be used for the model. Only one wind speed was used when testing the whole parametric space 
to minimize the test matrix size. A speed independence study was carried on and different speeds were tested with the boat base position showing minimal changes in the coefficients with speed, confirming the use of the one-speed hypothesis. The model obtained takes into account sail and windage forces, being a complete aerodynamic model of the boat.

IMS model:

The IMS sails model [33] can be used to determine sail forces and moments using the Moth main sail geometric dimensions. It is based on wind tunnel data (for the coefficient data used) in combination with an analytic model describing sails aerodynamics. If a specific Moth sail force model is not available this option is a good compromise solution when used with reef parameter deactivated in the trimming optimization loop.

\section{D.1.2 Windage}

Modified Windage Bögle et al. [18] model:

This model implements the original one by Bögle et al. [18] based on raw data from Beaver et al. paper [10] for crew and hull. It is completed to take into account emerged appendages below the hull, using foil sectional drag coefficients, and rig elements. Except for the interpolation of the foil sectional drag data, it can be considered as an analytic model.

\section{D.2 Hydrodynamic Models}

\section{D.2.1 Lifting Surfaces}

Elliptical distribution lifting line:

This is an analytical model combined with XFoil interpolated data of $2 \mathrm{D}$ foil lift and drag coefficients. The implemented equations D.1 and D.2 take into account 2D sectional lift coefficient for a given AoA instead of the CL slope. Drag is calculated using a chord-weighted strip method using the 2D drag coefficient for a given AoA.

$$
\begin{gathered}
C_{L 3 D}=C_{L 2 D}\left(\frac{\mathrm{AR}}{\mathrm{AR}+2}\right) \\
C_{D_{i}}=\frac{C_{L}{ }^{2}}{\pi \mathrm{AR}}
\end{gathered}
$$


Complex Lifting Line:

It is the simplest numerical model that can be calculated in execution time. It is based on the Lifting Line implementation that takes into account the hydrodynamic twist (camber induced AoA) distribution, the geometric twist distribution and the chord distribution using a station discretization of the foil. Two implementations of this method are available in PASim. A modern numerical implementation based on Multhopp coefficient approach [118] that uses the CL slope of the sections to obtain the three-dimensional lift coefficient. A non-linear implementation (recommended in high AoA or high flap angle cases) that uses non-linear section data with a modified version of the method presented by Sivells et al. in [161] using an iterative scheme based on the determination of the induced angle of attack distribution.

VLM implementations:

This is the most complex numerical model for lifting surface implemented that can be calculated in execution time.

One of three VLM codes available is based in QuadAir, a Matlab implementation of Bunge et al. work [22]. It combines a VLM for multiple lifting surfaces (that can have control surfaces) with a strip theory drag calculation. In a piercing foil situation, the foil is re-meshed using the underwater geometry and the calculation is executed for the new geometry. For a faster execution, a multiple foiling height matrix can be precomputed for the different foiling surfaces and then the force coefficients can be interpolated based on the precomputed data. For fully submerged appendages is the most computationally efficient implementation (the so-called aerodynamic coefficient matrix inversion is only done once).

Two more vortex ring VLM implementations for steady and unsteady calculations based on the work of Katz et al. [86] are also available in PASim. The unsteady implementation is suitable only for fully submerged appendages or fixed position piercing foils because the changing mesh with height methodology is no compatible with the wake meshing approach of this implementation.

End-plating and Piercing corrections: 
To take into account the effect of the piercing foils and end-plating using Lifting line and VLM methods, three different corrections have been implemented.

The first correction is related to the effective aspect ratio of the lifting surface. The real aspect ratio is multiplied by a constant fAR that depends on the foil situation (following the corrections presented in Bögle et al. [18]):

- When the foil is fully submerged $\mathrm{fAR}=2$, due to the end-plating effects of the hull.

- When the foil is piercing the surface fAR $=1$.

- When the foil bottom is end-plated by another foil $\mathrm{fAR}=1.5$.

If more than one of the situations described previously occurs at the same time, the value of fAR will be the product of the fAR values for each occurring situation (for example for a fully submerged foil end-plated at the bottom fAR $=3$ ).

The second correction is related to the appearance of two new components in the foil resistance. The first component is due to the wave train generated by the proximity of the horizontal foil to the free surface. The second component is related to the added resistance due to the foil piercing the free surface (spray generation, etc). The two drag components are added to the previously calculated foil drag, obtaining the total foil drag. This correction is based on the one presented in Bögle et al. [18].

The third correction is a vertical and horizontal foil interference model based on the foils section geometry characteristics near the intersection and proposed by Hoerner in his book "Fluid-Dynamic Drag: theoretical, experimental and statistical information" [77].

\section{D.2.2 Hull}

Hull model:

The model to generate the hull forces is an interpolated model using the hull resistance for different displacements data available in Beaver et al. paper [10]. The model is completed with the addition of the hydrostatic vertical force for each displacement situation. 


\section{D.3 Other Models}

\section{D.3.1 Control Models}

Modified version of Bögle et al. [18] Wand control model:

The model of the control wand is developed taking into account all the geometric relations presented in Bögle et al. [18] allowing the determination of the forces and moments acting on the wand (in the top and in the submerged bottom of the rod). Instead of using a simplified approach like the one used in Bögle et al., the implementation developed for this thesis uses a Newton scheme to find the equilibrium of forces and moments acting on the wand to obtain a certain ride height and flap angle of the main foil. Bögle et al. consider the force generated by the elastic rope acting on the top of the wand as constant, the implemented version in this thesis adds the possibility to calculate such force using Hooke law. It can also take into account the moment generated by the flap itself when solving the equilibrium of forces and moments. The hydrodynamic forces of the submerged part of the rod are calculated using a simple analytical model based on the rod drag coefficient and the speed of the incoming flow affected by the wand angle (cross-flow effect). A version of the model is also available for modern paddle wands systems based on pure geometrical aspects, where a paddled tip is added to the rod to read the water free surface independently of the rod immersion (as it is the case with a normal wand). This allows minimizing wand drag and reduce foil interaction and ventilation because of the wand proximity.

\section{D.3.2 Other Hydrostatics and Weight Stability Models}

Yacht and crew weight model:

The weights, CoG and inertias of all the yacht parts are included in the VPP preprocess. The final weight, CoG and inertia of the Moth are calculated when the VPP models are initialized. The weight of the crew is also taken into account. The $\mathrm{X}$ and $\mathrm{Y}$ coordinates of the CoG position of the crew are optimized during the VPP equilibrium calculation, the $\mathrm{Z}$ coordinate is calculated based on the boat geometry (hiking wings) and the two other coordinates.

Appendage Buoyancy model based on submerged volume calculation 
A specific model is implemented to calculate the piercing point of each foil (if the boat is foiling) and the submerged stations and foil characteristics. This information is used by a specific hydrostatic model that calculates submerged volume and the correspondent hydrostatic vertical force. 


\section{Appendix E}

\section{Washout Tuning Algorithm Results}

The following tables show the results of a simpler version of the washout tuning algorithm described in section 4.4.2.2. Each table shows the results for a specific Genetic algorithm for different populations sizes and number of generations. The platform geometry used is similar to the geometry of the SIROCCO hexapod from Symetrie [168].

The results highlighted in green are the ones presenting the lowest total maximum linear error. The ones highlighted in red are the results presenting higher total maximum linear error than the one obtained using the default tuning parameters for the classical algorithm presented in [151]. 
Table E.1: NSGAII algorithm results

\begin{tabular}{|c|c|c|c|c|c|c|c|c|}
\hline \multicolumn{9}{|c|}{ pNSGAII } \\
\hline \multicolumn{9}{|c|}{ Parallel Version of NSGAII } \\
\hline Population Size & Number of Generations & Total Max Error & Max Error X & Max Error Y & Max Error $\mathrm{Z}$ & Max Error Roll & Max Error Pitch & Max Error Yaw \\
\hline 50 & 100 & 1.652669423 & 0.72252212 & 0.43162714 & 0.11333069 & 0.03036654 & 0.05383008 & 0.30099285 \\
\hline 50 & 200 & 1.590055056 & 0.74745801 & 0.38921266 & 0.1227382 & 0.04694408 & 0.04123544 & 0.24246666 \\
\hline 50 & 500 & 1.625005565 & 0.69405071 & 0.39082173 & 0.10662743 & 0.04502444 & 0.04450868 & 0.34397259 \\
\hline 100 & 100 & 1.52503241 & 0.6965075 & 0.40077776 & 0.10892588 & 0.03561803 & 0.04166959 & 0.24153366 \\
\hline 100 & 200 & 1.661055663 & 0.67951967 & 0.45210606 & 0.10510895 & 0.07289506 & 0.03361023 & 0.31781568 \\
\hline 100 & 500 & 1.613482627 & 0.69101825 & 0.40875042 & 0.10571283 & 0.05212955 & 0.05121299 & 0.3046586 \\
\hline 200 & 100 & 1.675205911 & 0.76837977 & 0.39446547 & 0.10257264 & 0.04279405 & 0.04105886 & 0.32593512 \\
\hline 200 & 200 & 1.565368294 & 0.74865861 & 0.38717629 & 0.1133636 & 0.04468653 & 0.04040503 & 0.23107824 \\
\hline \multirow[t]{2}{*}{200} & 500 & 1.68585383 & 0.6696841 & 0.38818176 & 0.13286087 & 0.09294656 & 0.06837987 & 0.33380067 \\
\hline & Mean Error & 1.62152542 & & & & & & \\
\hline
\end{tabular}


Table E.2: MOEA/D algorithm results

pcMOEA/D With predefined Weight Vectors

Parallel Adapted Constrained Version of MOEA/D

\begin{tabular}{|r|r|r|r|r|r|r|r|r|r|}
\hline Population Size & Number of Generations & Total Max Error & Max Error X & Max Error Y & Max Error Z & Max Error Roll & Max Error Pitch & Max Error Yaw \\
\hline 126 & 126 & 1.534259422 & 0.69862792 & 0.40264108 & 0.12014203 & 0.04173719 & 0.04053604 & 0.23057516 \\
\hline 252 & 252 & 1.532801334 & 0.70002293 & 0.40121502 & 0.12750295 & 0.03509003 & 0.0403392 & 0.2286312 \\
\hline 462 & 462 & 1.531808915 & 0.69646309 & 0.39425312 & 0.13477039 & 0.03835169 & 0.0403758 & 0.22759483 \\
\hline 792 & 792 & 1.47354034 & 0.69444299 & 0.39989571 & 0.11810021 & 0.04304516 & 0.04191242 & 0.17614384 \\
\hline & & 1.518102503 & &
\end{tabular}


Table E.3: NSGAIII (outer dimension reference point generation only) algorithm results

\begin{tabular}{|c|c|c|c|c|c|c|c|c|}
\hline \multicolumn{9}{|c|}{ pNSGAIII With only outer dimension reference point generation } \\
\hline \multicolumn{9}{|c|}{ Parallel Version of NSGAIII } \\
\hline Population Size & Number of Generations & Total Max Error & Max Error $\mathrm{X}$ & Max Error $\mathrm{Y}$ & Max Error Z & Max Error Roll & Max Error Pitch & Max Error Yaw \\
\hline 128 & 200 & 1.529270098 & 0.69839242 & 0.39158082 & 0.10827183 & 0.0419166 & 0.04164374 & 0.24746468 \\
\hline 128 & 400 & 1.525620518 & 0.69567698 & 0.39631682 & 0.11241611 & 0.03910043 & 0.04126156 & 0.24084862 \\
\hline 128 & 800 & 1.53175702 & 0.69792568 & 0.39851812 & 0.11260227 & 0.03501413 & 0.04087842 & 0.24681838 \\
\hline 128 & 1200 & 1.532182168 & 0.69454886 & 0.39414345 & 0.11934923 & 0.04156552 & 0.04193493 & 0.24064017 \\
\hline
\end{tabular}


Table E.4: NSGAIII (inner and outer dimension reference point generation) algorithm results

\begin{tabular}{|c|c|c|c|c|c|c|c|c|}
\hline \multicolumn{9}{|c|}{ pNSGAIII With inner and outer dimension reference point generation } \\
\hline \multicolumn{9}{|c|}{ Parallel Version of NSGAIII } \\
\hline Population Size & Number of Generations & Total Max Error & Max Error X & Max Error Y & Max Error Z & Max Error Roll & Max Error Pitch & Max Error Yaw \\
\hline 132 & 200 & 1.548702305 & 0.69866945 & 0.39577113 & 0.11480299 & 0.03932658 & 0.04206601 & 0.25806615 \\
\hline 132 & 400 & 1.529406064 & 0.70328862 & 0.39297382 & 0.11009901 & 0.04051655 & 0.04100555 & 0.24152251 \\
\hline 132 & 800 & 1.554520778 & 0.70119947 & 0.40318937 & 0.13013213 & 0.03550544 & 0.04134757 & 0.2431468 \\
\hline 132 & 1200 & 1.510701022 & 0.69541437 & 0.39361965 & 0.11427625 & 0.03982293 & 0.0410029 & 0.22656492 \\
\hline 132 & 1600 & 1.540700194 & 0.70242085 & 0.39177474 & 0.12767788 & 0.04085213 & 0.04045 & 0.2375246 \\
\hline 132 & 2000 & 1.517832802 & 0.69321205 & 0.39424204 & 0.12049271 & 0.04065623 & 0.04052654 & 0.22870323 \\
\hline 132 & 3000 & 1.518388354 & 0.6933969 & 0.39448997 & 0.10820116 & 0.04028384 & 0.04130079 & 0.24071569 \\
\hline 132 & 4000 & 1.522805269 & 0.69199226 & 0.39750305 & 0.10584523 & 0.03866182 & 0.04108345 & 0.24771946 \\
\hline \multirow[t]{2}{*}{132} & 5000 & 1.524848087 & 0.70136381 & 0.38938865 & 0.12403551 & 0.04498529 & 0.04056974 & 0.2245051 \\
\hline & Mean Error & 1.529767208 & & & & & & \\
\hline
\end{tabular}




\section{Bibliography}

[1] D. Ariel and R. Sivan. False cue reduction in moving flight simulators. IEEE Transactions on Systems, Man, and Cybernetics, (4):665-671, 1984.

[2] Md. Asafuddoula, T. Ray, R. Sarker, and K. Alam. An adaptive constraint handling approach embedded moea/d. In Evolutionary Computation (CEC), 2012 IEEE Congress on, pages 1-8. IEEE, 2012.

[3] International Moth Class Association. International Moth class rules. "http://www.moth-sailing.org/wp-content/uploads/2017/05/ MTH2017CR010517 .pdf", 2017 (accessed December 11, 2017).

[4] International Moth Class Association. International Moth class rules and documents web page. "http://www.moth-sailing.org/history/ rules-and-documents/", 2017 (accessed December 11, 2017).

[5] C. A. Avizzano, P. Tripicchio, L. Joale, and M. Bergamasco. Design of a motion based sailing simulator. In RO-MAN, 2010 IEEE, pages 1-7. IEEE, 2010.

[6] D. D. Baals and W. R. Corliss. Wind tunnels of NASA, volume 440. Scientific and Technical Information Branch, National Aeronautics and Space Administration, 1981.

[7] M. Baarspul. The generation of motion cues on a six-degrees-of-freedom motion system. Delft University of Technology, Department of Aerospace Engineering, Report LR-248, 1977.

[8] T. J. Baker, A. Jameson, and W. Schmidt. A family of fast and robust Euler codes. Univ., 1984.

[9] F. Barbagli, D. Ferrazzin, C. A. Avizzano, and M. Bergamasco. Washout filter design for a motorcycle simulator. In Virtual Reality, 2001. Proceedings. IEEE, pages 225-232. IEEE, 2001. 
[10] Bill Beaver and John Zseleczky. Full scale measurements on a hydrofoil international Moth. In The 19th Chesapeake Sailing Yacht Symposium, 2009.

[11] S. Ben Hamed, W. Page, C. Duffy, and A. Pouget. Mstd neuronal basis functions for the population encoding of heading direction. Journal of neurophysiology, 90(2):549-558, 2003.

[12] J. R. Binns, F. W. Bethwaite, and N. R. Saunders. Development of a more realistic sailing simulator. In The 1st High Performance Yacht Design Conference, 2002 .

[13] J. R. Binns, N. Clark, T. Munro, C. Manzie, D. Oetomo, G. Bennett, N. Saunders, and M. Habgood. Verification and validation of an active sailing simulator. In Proceedings of the Asia-Pacific Simulation Training Conference 8 Exhibition, pages $18-21,2012$.

[14] J. R. Binns, K. Hochkirch, F. De Bord, and I. A. Burns. The development and use of sailing simulation for iacc starting manoeuvre training. In The 3rd High Performance Yacht Design Conference, 2008.

[15] F. Biocca, M. R. Levy, and J. Lawrence. Communication in the age of virtual reality. Psyccritiques, 42(2):170, 1997.

[16] M. J. Blackburn. A 90 Minute Dinghy Sailing Race Simulation : Physiological Responses to Discontinuous Isometric Exercise. PhD thesis, Honours Thesis, University of Canberra, 1991.

[17] M. J. Blackburn. Physiological responses to 90 min of simulated dinghy sailing. Journal of Sports Sciences, 12(4):383-390, 1994.

[18] C. Bögle, K. Hochkirch, H. Hansen, and G. Tampier-Brockhaus. Evaluation of the performance of a hydro-foiled Moth by stability and force balance criteria. Bachelor of Engineering Master's thesis, Tu-Berlin, 2010.

[19] H. Boterenbrood. Canopen high-level protocol for can-bus. Nikhef, Amsterdam, 2000.

[20] C. Bregler. Motion capture technology for entertainment [in the spotlight]. IEEE Signal Processing Magazine, 24(6):160-158, 2007. 
[21] K. H. Britten. Mechanisms of self-motion perception. Annu. Rev. Neurosci., 31:389-410, 2008.

[22] R. A. Bunge and I. M. Kroo. Compact formulation of nonlinear inviscid aerodynamics for fixed-wing aircraft. AIAA Paper, 2771:2012, 2012.

[23] J. Bürki-Cohen, A. L. Sparko, and T. H. Go. Training value of a fixed-base flight simulator with a dynamic seat. In AIAA Modelling and Simulation Technologies Conference, volume 6564, 2007.

[24] P. G. Bursztyn, S. Coleman, T. Hale, and J. Harrison. Laboratory simulation of the physical demands of single-handed dinghy racing. In JOURNAL OF PHYSIOLOGY-LONDON, volume 400, pages P14-P14. CAMBRIDGE UNIV PRESS 40 WEST 20TH STREET, NEW YORK, NY 10011-4211, 1988.

[25] M. Caponnetto, B. Bučan, M. Pedišić-Buča, M. Perić, and C. Pettinelli. Simulation of flow and motion of high-speed vessels. In Proceedings of the 12th International Conference on Fast Sea Transportation, 2013.

[26] G. F. Clauss and W. Heisen. Cfd analysis on the flying shape of modern yacht sails. In Maritime Transportation and Exploitation of Ocean and Coastal Resources: Proceedings of the 11th International Congress of the International Maritime Association of the Mediterranean, Lisbon, Portugal, page 87, 2006.

[27] D. Cleij, J. Venrooij, P. Pretto, M. Katliar, H. H. Bülthoff, D. Steffen, F. W. Hoffmeyer, and H-P Schöner. Comparison between filter-and optimizationbased motion cueing algorithms for driving simulation. Transportation Research Part F: Traffic Psychology and Behaviour, 2017.

[28] Bjorn Conrad and SF Schmidt. Motion drive signals for piloted flight simulators. 1970.

[29] M. V. Cook. Flight dynamics principles: a linear systems approach to aircraft stability and control. Butterworth-Heinemann, 2012.

[30] UNIGINE Corp. Unigine 2.2.1 Engine Documentation, 2016.

[31] UNIGINE Corp. Engine professional input and output. "https: //unigine.com/en/products/engine/professional-input-output", 2017 (accessed January 8, 2018). 
[32] HTC Corporation. Htc vive. "https://www.vive.com/us/product/ vive-virtual-reality-system/", 2017 (accessed January 8, 2018).

[33] Offshore Racing Council. Orc vpp documentation. Technical report, ORC, 2017.

[34] Nuno A Cruz and José C Alves. Auto-heading controller for an autonomous sailboat. In OCEANS 2010 IEEE-Sydney, pages 1-6. IEEE, 2010.

[35] C. Cruz-Neira, D. J. Sandin, and T. A. DeFanti. Surround-screen projectionbased virtual reality: the design and implementation of the cave. In Proceedings of the 20th annual conference on Computer graphics and interactive techniques, pages 135-142. ACM, 1993.

[36] J. J. Cummings and J. N. Bailenson. How immersive is enough? a metaanalysis of the effect of immersive technology on user presence. Media Psychology, 19(2):272-309, 2016.

[37] P. Cunningham and T. Hale. Physiological responses of elite laser sailors to 30 minutes of simulated upwind sailing. Journal of Sports Sciences, 25(10):11091116, 2007.

[38] M. Dagdelen, G. Reymond, A. Kemeny, M. Bordier, and N. Maïzi. Modelbased predictive motion cueing strategy for vehicle driving simulators. Control Engineering Practice, 17(9):995-1003, 2009.

[39] B. Dasgupta and T. S. Mruthyunjaya. Closed-form dynamic equations of the general stewart platform through the newton-euler approach. Mechanism and machine theory, 33(7):993-1012, 1998.

[40] B. Dasgupta and T. S. Mruthyunjaya. A newton-euler formulation for the inverse dynamics of the stewart platform manipulator. Mechanism and machine theory, 33(8):1135-1152, 1998.

[41] B. Dasgupta and T. S. Mruthyunjaya. The stewart platform manipulator: a review. Mechanism and machine theory, 35(1):15-40, 2000.

[42] K. S. M. Davidson. Some experimental studies of the sailing yacht. Society of naval architects and marine engineers, 1936. 
[43] K. Deb and H. Jain. An evolutionary many-objective optimization algorithm using reference-point-based nondominated sorting approach, part i: Solving problems with box constraints. IEEE Trans. Evolutionary Computation, 18(4):577$601,2014$.

[44] K. Deb, A. Pratap, S. Agarwal, and T. Meyarivan. A fast and elitist multiobjective genetic algorithm: Nsga-ii. IEEE transactions on evolutionary computation, 6(2):182-197, 2002.

[45] M. Derakhti, J. T. Kirby, F. Shi, and G. Ma. Nhwave: Model revisions and tests of wave breaking in shallow and deep water. Center for Applied Coastal Research, Department of Civil and Environmental Engineering. University of Delaware. Research Report No. CACR-15-18, 2015.

[46] J. Dichgans and T. Brandt. Visual-vestibular interaction: Effects on self-motion perception and postural control. In Perception, pages 755-804. Springer, 1978.

[47] M. Drela. Xfoil: An analysis and design system for low reynolds number airfoils. In Low Reynolds number aerodynamics, pages 1-12. Springer, 1989.

[48] Inc Epic Games. Unreal 4.12 Engine Documentation, 2016.

[49] B. Etkin and L. D. Reid. Dynamics of flight: stability and control, volume 3. Wiley New York, 1996.

[50] V. M. Falkner. The solution of lifting plane problems by vortex lattice theory. $R \&$ \& $M, 2591,1947$.

[51] R. Fernando. GPU Gems: Programming Techniques, Tips and Tricks for RealTime Graphics. Pearson Higher Education, 2004.

[52] E. F. Fichter. A stewart platform-based manipulator: general theory and practical construction. The International Journal of Robotics Research, 5(2):157-182, 1986.

[53] E. F. Fichter and E. D. McDowell. determining the motions of joints on a parallel connection manipulator. In Proc. 6th World Congress on Theory of Machines and Mechanisms, pages 1003-1006, 1983.

[54] E. Fix and H. G. Armstrong. Modeling human performance with neural networks. In Neural Networks, 1990., 1990 IJCNN International Joint Conference on, pages 247-252. IEEE, 1990. 
[55] R. G. J. Flay. A twisted flow wind tunnel for testing yacht sails. Journal of Wind Engineering and Industrial Aerodynamics, 63(1-3):171-182, 1996.

[56] R. G. J. Flay, N. J. Locke, and G. D. Mallinson. Development of a wind tunnel with twisted flow for testing yacht sails. In Ninth International Conference on Wind Engineering, volume 1, pages 275-286, 1995.

[57] F. Fossati, G. Mainetti, M. Malandra, R. Sala, P. Schito, and A. Vandone. Offwind sail flying shapes detection. In Proc. of the 5th High Performance Yacht Design Conference. Auckland, New Zealand, pages 48-59, 2015.

[58] T. I. Fossen. Guidance and control of ocean vehicles. John Wiley \& Sons Inc, 1994.

[59] T. I. Fossen. Handbook of marine craft hydrodynamics and motion control. John Wiley \& Sons, 2011.

[60] A. Fournier and W. T. Reeves. A simple model of ocean waves. ACM Siggraph Computer Graphics, 20(4):75-84, 1986.

[61] W. Froude, W. Abell, R. W. L. Gawn, and A. D. Duckworth. The Papers of William Froude, MA, LL. D., FRS, 1810-1879. Institution of Naval Architects, 1955.

[62] T. J. Gale and J. T. Walls. Development of a sailing dinghy simulator. Simulation, 74(3):167-179, 2000.

[63] N. J. I. Garrett and M. C. Best. Driving simulator motion cueing algorithms-a survey of the state of the art. In Proceedings of the 10th International Symposium on Advanced Vehicle Control (AVEC), Loughborough, UK, 22nd-26th August, pages 183-188, 2010.

[64] F. C. Gerhardt, R. G. J. Flay, and P. Richards. Unsteady aerodynamics of two interacting yacht sails in two-dimensional potential flow. Journal of Fluid Mechanics, 668:551-581, 2011.

[65] J. J. Gibson. The perception of the visual world. 1950.

[66] Crytek GmbH. CryEngine 5.10 Documentation, 2016.

[67] V.E. Gough and S. G. Whitehall. Universal tyre test machine. Proc. 9th Int. Technical Congr. FISITA, London, 1962, 1962. 
[68] J. Guermond. A generalized lifting-line theory for curved and swept wings. Journal of Fluid Mechanics, 211:497-513, 1990.

[69] J. Guermond and A. Sellier. A unified unsteady lifting-line theory. Journal of Fluid Mechanics, 229:427-451, 1991.

[70] D. R. Gum. Modeling of the human force and motion-sensing mechanics. Technical report, AIR FORCE HUMAN RESOURCES LAB BROOKS AFB TX, 1973.

[71] M. Hanada. An algorithmic model of heading perception. Biological cybernetics, 92(1):8-20, 2005.

[72] H. Hansen. Enhanced wind tunnel techniques and aerodynamic force models for yacht sails. PhD thesis, The University of Auckland, 2006.

[73] P. Hansen. Sailing simulator, February 28 1967. US Patent 3,305,943.

[74] Inc. Harken. Harken calcualtors. "http://www.harken.com/Calculators/", 2017 (accessed January 20, 2018).

[75] L. R. Harris, M. R. Jenkin, D. Zikovitz, F. Redlick, P. Jaekl, U. T. Jasiobedzka, H. L. Jenkin, and R. S. Allison. Simulating self-motion i: Cues for the perception of motion. Virtual Reality, 6(2):75-85, 2002.

[76] G. Hazen. Sailing yacht performance prediction, 1989.

[77] Sighard F Hoerner. Fluid-dynamic drag: theoretical, experimental and statistical information. Hoerner Fluid Dynamics, 1992.

[78] I. P. Howard. The perception of posture, self motion, and the visual vertical. Handbook of perception and human performance, pages 18-1, 1986.

[79] K. H. Hunt. Kinematic geometry of mechanisms, volume 7. Oxford University Press, USA, 1978.

[80] J.a Ish-Shalom et al. Design of optimal motion for flight simulators. PhD thesis, Massachusetts Institute of Technology, 1982.

[81] H. Ishibuchi, K. Doi, H. Masuda, and Y. Nojima. Relation between weight vectors and solutions in moea/d. In Computational Intelligence, 2015 IEEE Symposium Series on, pages 861-868. IEEE, 2015. 
[82] J. Isidoro, A. Vlachos, and C. Brennan. Rendering ocean water. Direct3D ShaderX: Vertex and Pixel Shader Tips and Tricks, Wordware, 2002.

[83] P. Izaguirre Alza. Numerical and Experimental Studies of Sail Aerodynamics. PhD thesis, Technical University of Madrid (UPM), 2012.

[84] H. Jain and K. Deb. An evolutionary many-objective optimization algorithm using reference-point based nondominated sorting approach, part ii: Handling constraints and extending to an adaptive approach. IEEE Trans. Evolutionary Computation, 18(4):602-622, 2014.

[85] R. T. Jones. The unsteady lift of a wing of finite aspect ratio. 1940.

[86] J. Katz and A. Plotkin. Low-speed aerodynamics, volume 13. Cambridge university press, 2001.

[87] J. E. Kerwin. A velocity prediction program for ocean racing yachts. In New England Sailing Yacht Symposium, 1976.

[88] J. E. Kerwin and J. N. Newman. A summary of the h. irving pratt ocean race handicapping project. In Proceedings of the 4 th Chesapeake Sailing Yacht Symposium, 1979.

[89] J. A. Keuning and M. Katgert. A bare hull resistance prediction method derived from the results of the delft systematic yacht hull series extended to higher speeds. In International Conference on Innovation in High Performance Sailing Yachts, Lorient, France, 2008.

[90] J. A. Keuning, K. J. Vermeulen, and E. J. De Ridder. A generic mathematical model for the maneuvering and tacking of a sailing yacht. In The 17th Chesapeake Sailing Yacht Symposium, pages 143-163, 2005.

[91] D. L. Key et al. Fidelity of simulation for pilot training. AGARD Advisory Report, 159, 1980.

[92] S. Khan and R. Chang. Anatomy of the vestibular system: a review. NeuroRehabilitation, 32(3):437-443, 2013.

[93] M. Kikuchi and J. Takashina. Development of marine simulator for singlehanded sailboat. In $M C M C^{\prime} 94$, pages 287-300, 1994. 
[94] F. W. Lanchester. Aerodynamics. Constable \& Co Ltd., London, England, 1907.

[95] M. E. Latoschik. Realtime 3d computer graphics virtual reality. Artificial Intelligence Group, University of Bielefeld, 2006.

[96] D. J. Le Pelley and O. Modral. V-spars: A combined sail and rig shape recognition system using imaging techniques. In Proc. of the 3rd High Performance Yacht Design Conference. Auckland, New Zealand, pages 2-4, 2008.

[97] J. G. Leishman. Principles of helicopter aerodynamics, 2006. Cambridge Aerospace Series.

[98] F. W. Lewis, S. Jagannathan, and A. Yesildirak. Neural network control of robot manipulators and non-linear systems. CRC Press, 1998.

[99] A. K. Lidtke, L. Marimon Giovanetti, L. M. Breschan, A. Sampson, M. Vitti, and D. Taunton. Development of an america's cup 45 tacking simulator. In The Third International Conference on Innovation in High Performance Sailing Yachts, Lorient, France, 2013.

[100] M. Lombard and T. Ditton. At the heart of it all: The concept of presence. Journal of Computer-Mediated Communication, 3(2):0-0, 1997.

[101] Vicon Motion Systems Ltd. Vicon vantage. "https://www.vicon.com/ products/camera-systems/vantage", 2017 (accessed January 10, 2018).

[102] C. A. Marchaj. Aero-hydrodynamics of sailing. Adlard Coles Nautical, 1988.

[103] Y. Masuyama, T. Fukasawa, and H. Sasagawa. Tacking simulation of sailing yachts-numerical integration of equations of motion and application of neural network technique. In The Twelfth Chesapeake Sailing Yacht Symposium, 1995, 1, pages $117-131,1995$.

[104] K. Matsumiya and H. Ando. World-centered perception of 3d object motion during visually guided self-motion. Journal of Vision, 9(1):15-15, 2009.

[105] J. Mausolf, J. Deparday, K. Graf, H. Renzsch, and C. Böhm. Photogrammetry based flying shape investigation of downwind sails in the wind tunnel and at full scale on a sailing yacht. In 20th Chesapeake Sailing Yacht Symposium, 2011. 
[106] Fraunhofer IPA Max Planck Institute for Biological Cybernetics (MPI). Cable-driven parallel robots motion simulation in a new dimension, press release. "https://www.ipa.fraunhofer.de/content/dam/ipa/de/ documents/Presse/Presseinformationen/2015/September/2015_09_09_ PM_Seilsimulator_MPI_IPA_en.pdf”, 2017 (accessed December 14, 2017).

[107] L. Mazas, Y. Andrillon, A. Letourneur, P. Kerdraon, and G. Verdier. Comparison of hydrodynamic performances of an imoca 60 with straight or l-shaped daggerboard. In INNOV'SAIL 2017, The Fourth International Conference on Innovation in High Performance Sailing Yachts, Lorient, France, 2017.

[108] H. McCallion and P. D. Truong. The analysis of a six-degree-of-freedom work station for mechanised assembly. In The Fifth World Congr. for the Theory of Machines and Mechanisms, ASME, New York, pages 611-616, 1979.

[109] M. E. McCauley and T. J. Sharkey. Cybersickness: Perception of self-motion in virtual environments. Presence: Teleoperators \& Virtual Environments, $1(3): 311-318,1992$.

[110] D. McLean. Automatic flight control systems. Englewood Cliffs, NJ, Prentice Hall, 1990, 606, 1990.

[111] E. McLoughlin, T. Hale, J. H. Harrison, and P. A. Keen. The effects of dietary manipulation on physiological responses to a 30 minute sailing task. Medical Science Research, 21:869, 1993.

[112] J. L. Meiry. The vestibular system and human dynamic space orientation. Technical Report CR-628, NASA, 1966.

[113] Mercedes-Benz. The mercedes-benz driving simulation center. "https://www.mercedes-benz.com/en/mercedes-benz/innovation/ the-mercedes-benz-driving-simulation-center/", 2017 (accessed December 14, 2017).

[114] J. P. Merlet. Research report 646. INRIA, I, 987, 1987.

[115] J. Mooney, N. R. Saunders, M. Habgood, and J. R. Binns. Multiple applications of sailing simulation. In Proceedings of SimTecT2009 Conference, Adelaide, Australia, 2009. 
[116] F. A. Mulder and J. C. Verlinden. Development of a motion system for an advanced sailing simulator. Procedia Engineering, 60:428-434, 2013.

[117] F. A. Mulder, J. C. Verlinden, and R. R. Dukalski. The effect of motion on presence during virtual sailing for advanced training. Presence 2012 ISPR 2012, Philadelphia (USA), 24-26 Oct., 2012, 2012.

[118] H. Multhopp. Die berechnung der auftriebsverteilung von tragflügeln, luftfahrtforschung bd. 15, nr.14. pages 153-169, 1938.

[119] F. Muñoz, M. Bonilla, I. González-Hernández, S. Salazar, and R. Lozano. Super twisting vs modified super twisting algorithm for altitude control of an unmanned aircraft system. In Electrical Engineering, Computing Science and Automatic Control (CCE), 2015 12th International Conference on, pages 1-6. IEEE, 2015.

[120] M. A. Nahon and L. D. Reid. Simulator motion-drive algorithms: A designers perspective. J. Guidance, 13(2):356-362, 1990.

[121] Inc Natural Point. Optitrack. "http://www.optitrack.com", 2017 (accessed January 10, 2018).

[122] A. J. Nebro and J. J. Durillo. A study of the parallelization of the multiobjective metaheuristic moea/d. LION, 4:303-317, 2010.

[123] K. Nishimura. Sailing simulator, March 2 1976. US Patent 3,940,862.

[124] D. Ocaña-Blanco, I. Castañeda-Sabadell, and A. Souto-Iglesias. Cfd and potential flow assessment of the hydrodynamics of a kitefoil. Ocean Engineering, 146:388-400, 2017.

[125] LLC. Oculus VR. Occulus rift. "https://www. oculus.com/rift/", 2017 (accessed January 8, 2018).

[126] R. V. Parrish, James E. D., and D. J. Martin Jr. Coordinated adaptive washout for motion simulators. Journal of aircraft, 12(1):44-50, 1975.

[127] R. V. Parrish, J. E. Dieudonne, and D. J. Martin Jr. Motion software for a synergistic six-degree-of-freedom motion base. 1973. NASA TN D-7350, NASA Langley Research Center, Hampton, VA. 
[128] Russell V Parrish and Dennis J Martin Jr. Comparison of a linear and a nonlinear washout for motion simulators utilizing objective and subjective data from ctol transport landing approaches. Technical Report D-8157, NASA, 1976.

[129] B. L. Parsons. Experimental techniques for model yacht performance evaluation-a review. Oceanic Engineering International, 2(1):1-18, 1998.

[130] D. R. Peachey. Modeling waves and surf. In ACM Siggraph Computer Graphics, volume 20, pages 65-74. ACM, 1986.

[131] T. Perez. Ship motion control: course keeping and roll stabilisation using rudder and fins. Springer Science \& Business Media, 2006.

[132] J. A. Perrone and L. S. Stone. A model of self-motion estimation within primate extrastriate visual cortex. Vision research, 34(21):2917-2938, 1994.

[133] J. A. Perrone and L. S. Stone. Emulating the visual receptive-field properties of mst neurons with a template model of heading estimation. Journal of Neuroscience, 18(15):5958-5975, 1998.

[134] D. W. Pessen. A new look at pid-controller tuning. Journal of dynamic systems, measurement, and control, 116(3):553-557, 1994.

[135] R. A. Peters. Dynamics of the vestibular system and their relation to motion perception, spatial disorientation, and illusions. Technical Report CR-1309, NASA, 1969.

[136] M. Pharr and R. Fernando. Gpu gems 2: programming techniques for highperformance graphics and general-purpose computation. Addison-Wesley Professional, 2005.

[137] Phasespace. Phasespace impulse x2e. "http://phasespace.com/ x2e-motion-capture/", 2017 (accessed January 10, 2018).

[138] W. F. Phillips. Lifting-line analysis for twisted wings and washout-optimized wings. Journal of aircraft, 41(1):128-136, 2004.

[139] W. F. Phillips and D. O. Snyder. Modern adaptation of prandtl's classic liftingline theory. Journal of Aircraft, 37(4):662-670, 2000. 
[140] A. Philpott and A. Mason. Advances in optimization in yacht performance analysis. In The 1st High Performance Yacht Design Conference, pages 229236, 2002.

[141] Jon Postel. User datagram protocol. Technical report, 1980.

[142] L. Prandtl. Tragflgeltheorie. Knigliche Gesellschaft der Wissenschaften zu Gttingen, 1918.

[143] W. H. Press, S. A. Teukolsky, W. T. Vetterling, and B. P. Flannery. Numerical recipes 3rd edition: The art of scientific computing, volume 3. Cambridge university press Cambridge, 2007.

[144] K. Priandana, W. Wahab, and B. Kusumoputro. Comparison of neural networks based direct inverse control systems for a double propeller boat model. In Proceedings of the Fifth International Conference on Network, Communication and Computing, pages 310-315. ACM, 2016.

[145] PS-Tech. Pst iris. "http://www.ps-tech.com/optical-trackers/ optical-tracker-pst-iris", 2017 (accessed January 10, 2018).

[146] B. Pueo and J. M. Jimenez-Olmedo. Application of motion capture technology for sport performance analysis. RETOS. Nuevas Tendencias en Educación Física, Deporte y Recreación, (32), 2017.

[147] R. Ranzenbach and J. Kleene. Utility of flying shapes in the development of offwind sail design databases. In Proc. 2nd High Performance Yacht Design Conference. Auckland, New Zealand, 2002.

[148] H. C. Raven. A solution method for the nonlinear ship wave resistance problem. HC Raven Marin, 1996.

[149] A. J. Rehmann, R. D. Mitman, and M. C. Reynolds. A handbook of flight simulation fidelity requirements for human factors research. Technical report, CREW SYSTEM ERGONOMICS INFORMATION ANALYSIS CENTER WRIGHTPATTERSON AFB OH, 1995.

[150] L. D. Reid and M. A. Nahon. Flight simulation motion-base drive algorithms: Part 1 - developing and testing equations. Technical Report 296, University of Toronto, 1985. 
[151] L. D. Reid and M. A. Nahon. Flight simulation motion-base drive algorithms.: Part 2 - selecting the system parameters. Technical Report 307, University of Toronto, 1986.

[152] L.D Reid and M. A. Nahon. Flight simulation motion-base drive algorithms. part 3 - pilot evaluations. Technical Report 319, University of Toronto, 1986.

[153] H. Renzsh and K. Graf. Fluid structure interaction simulation of spinnakers getting closer to reality. In International Conference on Innovation in High Performance Sailing Yachts, Lorient, France, 2010.

[154] C. S. Royden. Mathematical analysis of motion-opponent mechanisms used in the determination of heading and depth. JOSA A, 14(9):2128-2143, 1997.

[155] C. S. Royden, J. A. Crowell, and M. S. Banks. Estimating heading during eye movements. Vision research, 34(23):3197-3214, 1994.

[156] M. Ruiz de Elvira. Design of a generalized tool for the performance assessment under sail based on analytical, numerical and empirical results. A Global sailing yacht Meta-Model. PhD thesis, Technical University of Madrid (UPM), 2015.

[157] North Sails. North sails design suite. "https://northsails.com/sailing/en/ innovation/design-expertise/design-suite", 2017 (accessed December 9, 2017).

[158] M. Scarponi, R. A. Shenoi, S. R. Turnock, and P. Conti. Interactions between yacht-crew systems and racing scenarios combining behavioural models with vpps. In 19th International HISWA Symposium on Yacht Design and Yacht Construction, pages 109-120, 2006.

[159] J. A. Schroeder. Helicopter flight simulation motion platform requirements. Technical Report TP-1999-208766, NASA, 1999.

[160] R. Sivan, J. Ish-Shalom, and J. Huang. An optimal control approach to the design of moving flight simulators. IEEE Transactions on Systems, Man, and Cybernetics, 12(6):818-827, 1982.

[161] J. C. Sivells and R. H. Neely. Method for calculating wing characteristics by lifting-line theory using nonlinear section lift data. Technical report, NATIONAL ADVISORY COMMITTEE FOR AERONAUTICS LANGLEY FIELD VA LANGLEY AERONAUTICAL LABORATORY, 1947. 
[162] J. A. Somolinos Sánchez. Modelado dinámico y control de un robot flexible de tres grados de libertad. Univ. de Castilla La Mancha, 2001.

[163] B. L. Stevens, F. L Lewis, and E. N. Johnson. Aircraft control and simulation: dynamics, controls design, and autonomous systems. John Wiley \& Sons, 2015.

[164] D. Stewart. A platform with six degrees of freedom. Proceedings of the institution of mechanical engineers, 180(1):371-386, 1965.

[165] J. E. Stewart, D. M. Johnson, and W. R. Howse. Fidelity requirements for army aviation training devices: Issues and answers. Technical report, ARMY RESEARCH INST FOR THE BEHAVIORAL AND SOCIAL SCIENCES FORT RUCKER AL, 2008.

[166] R. Storn and K. Price. Differential evolution-a simple and efficient heuristic for global optimization over continuous spaces. Journal of global optimization, 11(4):341-359, 1997.

[167] I. E. Sutherland. A head-mounted three dimensional display. In Proceedings of the December 9-11, 1968, fall joint computer conference, part I, pages 757-764. ACM, 1968.

[168] Symetrie. Sirocco motion platform. "http://www.symetrie.fr/en/products/ motion-hexapods/sirocco/", 2018 (accessed May 15, 2018).

[169] T. Tanner. The geometry of sailing to windward. Trans. RINA, 1960.

[170] R. M. Taylor II, T. C. Hudson, A. Seeger, H. Weber, J. Juliano, and A. T. Helser. Vrpn: a device-independent, network-transparent vr peripheral system. In Proceedings of the ACM symposium on Virtual reality software and technology, pages 55-61. ACM, 2001.

[171] Unity Technologies. Unity 5.3.2 Engine Documentation, 2016.

[172] Jerry Tessendorf. Simulating ocean water. 2004.

[173] Theodore Theodorsen and WH Mutchler. General theory of aerodynamic instability and the mechanism of flutter. 1935.

[174] G. D. Thiart. Vortex lattice method for a straight hydrofoil near a free surface. International shipbuilding progress, 44(437):5-26, 1997. 
[175] G. D. Thiart. Generalized vortex lattice method for prediction of hydrofoil characteristics. RESD Journal, 2001.

[176] E. Thöndel. Design and optimisation of a motion cueing algorithm for a truck simulator. In 26th annual European Simulation and Modelling Conference, volume 22, page 10, 2012.

[177] International Moth Class Association UK. Foiling history. "http://www. internationalmoth.co.uk/the-moth/foiling-history/", 2017 (accessed December 11, 2017).

[178] B. G. van der Wall and J. G. Leishman. On the influence of time-varying flow velocity on unsteady aerodynamics. Journal of the American Helicopter Society, 39(4):25-36, 1994.

[179] M. Van Dyke. Perturbation methods in fluid mechanics/annotated edition. NASA STI/Recon Technical Report A, 75, 1975.

[180] J. C. Verlinden, F. A. Mulder, J. S. Vergeest, A. de Jonge, D. Krutiy, Z. Nagy, B. J. Logeman, and P. Schouten. Enhancement of presence in a virtual sailing environment through localized wind simulation. Procedia Engineering, 60:435441, 2013.

[181] R. S. Waddington, B. Duval, and S. D. Buckley. Simulating apparatus for teaching the art of sailing, August 10 1971. US Patent 3,597,856.

[182] H. Wagner. Über die entstehung des dynamischen auftriebes von tragflügeln. ZAMM-Journal of Applied Mathematics and Mechanics/Zeitschrift für Angewandte Mathematik und Mechanik, 5(1):17-35, 1925.

[183] J. Walls, L. Bertrand, T. Gale, and N. Saunders. Assessment of upwind dinghy sailing performance using a virtual reality dinghy sailing simulator. Journal of science and medicine in sport, 1(2):61-72, 1998.

[184] J. T. Walls and N. R. Saunders. Evolution of the dinghy simulator, a tool for sports specific testing. In Proceedings of the Australian Physiological and Pharmacological Society, volume 25, 1994.

[185] P. A. Warren and S. K. Rushton. Perception of object trajectory: parsing retinal motion into self and object movement components. Journal of Vision, $7(11): 2-2,2007$. 
[186] P. A Warren and S. K. Rushton. Evidence for flow-parsing in radial flow displays. Vision research, 48(5):655-663, 2008.

[187] R. B. Welch. Perceptual modification: Adapting to altered sensory environments. Academic Press, 1978.

[188] R. B. Welch. Adaptation of space perception. Handbook of perception and human performance, 1986.

[189] M. Wexler. Voluntary head movement and allocentric perception of space. Psychological Science, 14(4):340-346, 2003.

[190] M. Wexler and J. JA Van Boxtel. Depth perception by the active observer. Trends in cognitive sciences, 9(9):431-438, 2005.

[191] G. L. Zacharias. Motion cue models for pilot-vehicle analysis. Technical report, BOLT BERANEK AND NEWMAN INC CAMBRIDGE MA CONTROL SYSTEMS DEPT, 1978.

[192] R. Zamora-Rodriguez, P. Gomez-Alonso, J. Amate-Lopez, V. De-Diego-Martin, P. Dinoi, A. N. Simos, and A. Souto-Iglesias. Model scale analysis of a tlp floating offshore wind turbine. In ASME 2014 33rd International Conference on Ocean, Offshore and Arctic Engineering. American Society of Mechanical Engineers, 2014. 